University of Rhode Island

DigitalCommons@URI

Open Access Dissertations

2017

\title{
Using the Energy of Membrane-Associated Folding for Tumor Targeting and Intracellular Cargo Delivery
}

Linden C. Wyatt

University of Rhode Island, wyatt.lind@gmail.com

Follow this and additional works at: https://digitalcommons.uri.edu/oa_diss

\section{Recommended Citation}

Wyatt, Linden C., "Using the Energy of Membrane-Associated Folding for Tumor Targeting and Intracellular Cargo Delivery" (2017). Open Access Dissertations. Paper 630.

https://digitalcommons.uri.edu/oa_diss/630

This Dissertation is brought to you for free and open access by DigitalCommons@URI. It has been accepted for inclusion in Open Access Dissertations by an authorized administrator of DigitalCommons@URI. For more information, please contact digitalcommons-group@uri.edu. 
USING THE ENERGY OF MEMBRANE-ASSOCIATED FOLDING FOR TUMOR

TARGETING AND INTRACELLULAR CARGO DELIVERY

BY

LINDEN C. WYATT

A DISSERTATION SUBMITTED IN PARTIAL FULFILLMENT OF THE

REQUIREMENTS FOR THE DEGREE OF

DOCTOR OF PHILOSOPHY

IN

PHYSICS

UNIVERSITY OF RHODE ISLAND

2017 


\title{
DOCTOR OF PHILOSOPHY DISSERTATION
}

$\mathrm{OF}$

\author{
LINDEN C. WYATT
}

APPROVED:

Dissertation Committee:

Major Professor

Yana K. Reshetnyak

Oleg A. Andreev

Mindy Levine

Nasser H. Zawia

DEAN OF THE GRADUATE SCHOOL

UNIVERSITY OF RHODE ISLAND

2017 


\begin{abstract}
Cancer is the second leading cause of death in the United States. Due to the increase in exposure to cancer-causing environmental factors, the presence of chronic conditions, and the increase in age-related mutations in normal tissue, cancer incidence is positively correlated with age. Because of the increase in life expectancy due to advances in medicine, the frequency of cancer incidence is expected to rise in the future. For these reasons, devising methods to efficiently diagnose and treat cancer is extremely important.
\end{abstract}

Effective targeting of cancer cells within tumors would allow for improvement in the efficacy of treatment and the reduction of side effects. Tumor targeting falls into three broad categories: passive targeting, active targeting, and physical targeting. Passive targeting generally employs the enhanced permeability and retention effect, a phenomenon that leads to the localization of a certain range of sizes of macromolecules in tumor tissue due to the non-intact vasculature present in this tissue. Passive targeting can work well to deliver some therapeutic agents to solid tumors, but lacks targeting specificity and can therefore result in substantial targeting of healthy tissue, especially the liver, lymph nodes, and spleen.

Active targeting relies on a much greater presence of certain characteristics, called biomarkers, on or in cancer cells than on/in normal cells. To exploit active targeting, therapeutic agents are conjugated to targeting moieties like antibodies, aptamers, or small molecules that have a high affinity to these biomarkers. Active targeting has 
some advantages over passive targeting, namely, higher targeting specificity. However, one significant disadvantage of active targeting is its reliance on the overexpression of biomarkers in cancer cells: tumor tissue is very heterogeneous, with varying levels of expression across the cancer cell population; additionally, these biomarkers are usually present in normal tissue, leading to the undesirable targeting of healthy tissue.

Physical targeting relies on a difference in physical properties, such as temperature, oxygenation, or $\mathrm{pH}$, between normal tissue and cancer tissue. These physical characteristics can be more universal to tumor tissue, regardless of the tissue vasculature or biomarker expression. Physical targeting offers specificity advantages over passive targeting, and offers advantages over active targeting based on the universality and specificity of the targeted physical traits, compared to the broader, varying levels of expression of active-targeted biomarkers.

It has been known for over fifty years that tumor tissue undergoes acidification due to a switch in metabolic pathway resulting in the production of high levels of lactic acid and protons, a phenomenon known as the Warburg effect. In addition, cancer cells overexpress certain membrane proteins that catalyze reactions in the blood that result in further acidification. Although cell surface acidity is a feature that is ubiquitous to solid tumors, as a biomarker it has been greatly overlooked in favor of expressed traits like growth factors, protein receptors, and antigens. 
$\mathrm{pH}$ Low Insertion Peptides (pHLIPs) comprise a family of $\mathrm{pH}$-sensitive peptides, and sense and target the acidity present at the surface of cancer cells. pHLIPs can be conjugated to many different types of imaging and therapeutic agents, and, targeting the physical characteristic of $\mathrm{pH}$, are able to selectively deliver these agents to tumor tissue while avoiding normal tissue. The mechanism of action of pHLIP is based on membrane-associated folding triggered by low $\mathrm{pH}$ : at low $\mathrm{pH}$, a high concentration of protons results in the protonation of certain residues in pHLIP which leads to an increase in peptide hydrophobicity and drives the peptide to partition into the hydrophobic core of the membrane; here, pHLIP folds into a transmembrane helix, leaving one terminus in the extracellular space while the other terminus is translocated across the cell membrane into the cytoplasm.

The main goal of this work was to optimize various pHLIPs and to evaluate the utility of pHLIP conjugates in the selective delivery of imaging and therapeutic agents used in specific medical applications: namely, for PET imaging and for the intracellular delivery of toxic cargo. Over the course of this work, we introduced several new imaging and drug-delivery conjugates based on existing pHLIP variants, as well as several new drug-delivery conjugates based on novel pHLIP variants. An emphasis was placed on the biophysical characterization of new peptides and conjugates, and the measurement of the ability of drug-laden conjugates to induce cell death in in vitro assays in a pH-dependent manner. Aside from these points upon which we place special emphasis, the effects of physiological levels of free divalent cations on protein folding were investigated via biophysical measurements for the first time. 


\section{ACKNOWLEDGMENTS}

I am very grateful to my advisor, Dr. Yana K. Reshetnyak, for her guidance: the amount of time and energy that she spent working with me over the past five years is enormous and I am sure that were it not for her, my graduate career would have ended much earlier and much less propitiously. The amount of time and care that Dr. Reshetnyak dedicates to her graduate students is truly awesome and it amazes me that, given the amount of time that she dedicates to guiding her advisees, she is still such a productive member of the Physics Department, both in research and in teaching. Aside from being grateful to her for constantly guiding me and motivating me when I needed it most, I am very happy to have been able to share interesting and enjoyable conversations with Dr. Reshetnyak, and for her very pleasant sense of humor-an exception in physics much more than it is the rule. This sense of humor is shared with Dr. Oleg A. Andreev, another outstanding mentor who I feel privileged to have been able to work with. Conversations with Dr. Andreev have always been enlightening, productive, and enjoyable, and I have never ceased being amazed by his seemingly endless knowledge.

I am indebted to Dr. Anna Moshnikova, who spent a great amount of her time teaching me lab techniques and experimental techniques, repeatedly explaining various chemical reactions to me, and running experiments with me. Interacting with Anna is always pleasant, and I am very grateful for her patience with me over the last five years. Without Anna, I would have spent a very long time looking for answers in the wrong locations. I am also very grateful to Dr. Dhammika Weerakkody, who spent a 
good deal of time teaching me how to run and analyze biophysical experiments, and to the rest of the Division of Biological and Medical Physics for interesting and fruitful conversations.

I would like to thank Dr. Mindy Levine and Dr. Stephen M. Kennedy for serving on my graduate committee, particularly for the time they spent working with me and for fruitful and pleasant conversations over the course of my comprehensive and dissertation processes.

I owe a great deal of thanks to the faculty of the Physics Department of the University of Rhode Island, who have taught and guided me through undergraduate and graduate school. In particular, I am very grateful to Dr. David Heskett for his guidance through my undergraduate time and to Dr. Alexander Meyerovich for advising me in my first year of research, a year that I really enjoyed and that opened the door to graduate school for me. I am thankful to Dr. Gerhard Müller for dedicating a lot of time and energy to teaching many different fields of physics in an engaging and enjoyable manner (and for being an extremely entertaining, kind, and pleasant person), to Steve Pellegrino for many enjoyable conversations and for helping with my teaching responsibilities so much, and to Linda Connell for being so pleasant and helpful, and for working hard to make the department a much nicer and friendlier place.

Finally, I owe an enormous amount of thanks and gratitude to my family for their endless support and guidance, especially to my parents, to my grandparents, to the 
Juice, to my awesome sister Meredith, and to my closest friends: SMD and MIM; to MF, SMK, and MAK; and to my third family. And most importantly of all, to the ol' Beezer and to the Lord of the Morganites for putting up with me. 


\section{PREFACE}

This dissertation is written in "Manuscript" format, using the Thesis/Dissertation template of the University of Rhode Island. There are three manuscripts included in this dissertation, each of which comprises a chapter. The tables and figures of each manuscript are listed under the corresponding chapter in the list of tables and figures.

The results of our studies presented in the first two chapters were published in the following papers:

1. Wyatt LC, Lewis JS, Andreev OA, Reshetnyak YK, Engelman DM. "Applications of pHLIP Technology for Cancer Imaging and Therapy." (2017) Trends in Biotechnology. 35(7)653-664.

2. Demoin DW, Wyatt LC, Edwards KJ, Abdel-Atti D, Sarparanta M, Pourat J, Longo VA, Carlin SD, Engelman DM, Andreev OA, Reshetnyak YK, ViolaVillegas N, Lewis JS. (2016) "PET imaging of extracellular pH in tumors with ${ }^{64} \mathrm{Cu}$ - and ${ }^{18}$ F-labeled pHLIP peptides: A structure-activity optimization study." Bioconjugate Chemistry 27(9):2014-2023.

The final chapter is composed of research that has been submitted for publication:

3. Wyatt LC, Moshnikova A, Crawford T, Engelman DM, Andreev OA, Reshetnyak YK. "New pHLIPs for the Targeted Intracellular Delivery of Cargo Molecules to Tumors." Manuscript submitted for publication. 


\section{TABLE OF CONTENTS}

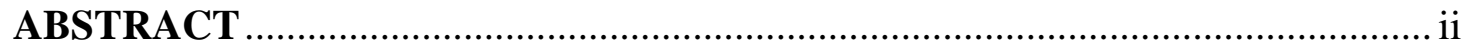

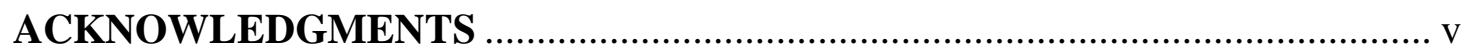

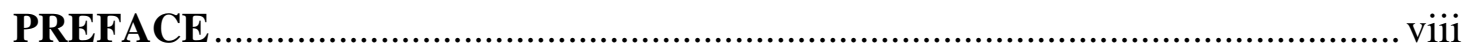

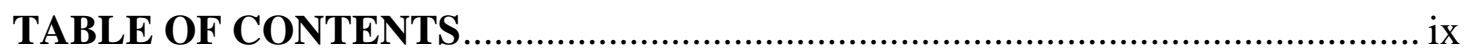

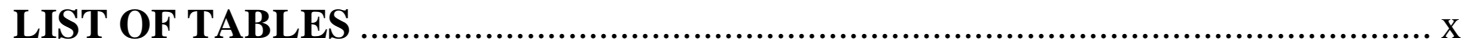

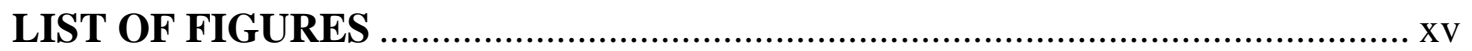

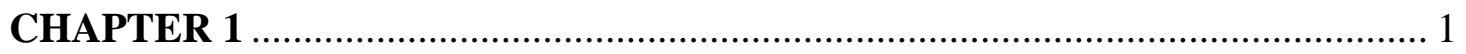

Applications of pHLIP Technology for Cancer Imaging and Therapy ................................ 1

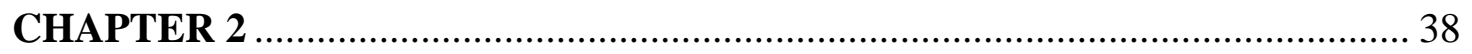

PET Imaging of Extracellular $\mathrm{pH}$ in Tumors with ${ }^{64} \mathrm{Cu}$ - and ${ }^{18} \mathrm{~F}$ - labeled pHLIP ${ }^{\circledR}$ Peptides:

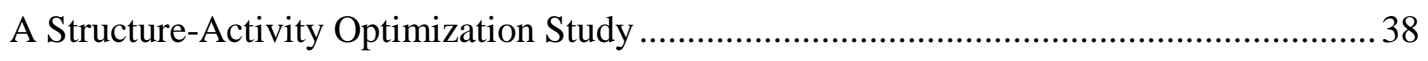

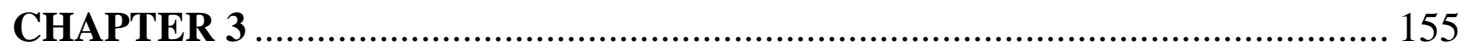

New pHLIPs for the Targeted Intracellular Delivery of Cargo Molecules to Tumors ........ 155 


\section{LIST OF TABLES}

\section{CHAPTER 1}

There are no tables in Chapter 1.

\section{CHAPTER 2}

Table 1. Biodistribution of N-terminus derivatized constructs 73

Table 2. Biodistribution of cysteine derivatized constructs

Table S1. Specific activities of tracers

Table S1-1. Tissue uptake (mean \%ID $\pm \mathrm{SD}$ ) of ${ }^{64} \mathrm{Cu}-\mathrm{NOTA}-\mathrm{WT}$ in BALB/c mice with orthotopic $4 \mathrm{~T} 1$ breast cancer allografts 92

Table S1-2. Tissue uptake (mean \%ID/g \pm SD) of ${ }^{64} \mathrm{Cu}-\mathrm{NOTA}-\mathrm{WT}$ in BALB/c mice with orthotopic $4 \mathrm{~T} 1$ breast cancer allografts 93

Table S2-1. Tissue uptake (mean \%ID $\pm \mathrm{SD}$ ) of ${ }^{64} \mathrm{Cu}-\mathrm{NOTA}-\mathrm{Var} 3$ in BALB/c mice with orthotopic $4 \mathrm{~T} 1$ breast cancer allografts 96

Table S2-2. Tissue uptake (mean \%ID/g $\pm \mathrm{SD}$ ) of ${ }^{64} \mathrm{Cu}-\mathrm{NOTA}-\operatorname{Var} 3$ in BALB/c mice with orthotopic 4T1 breast cancer allografts 97

Table S3-1. Tissue uptake (mean \% ID \pm SD) of ${ }^{64} \mathrm{Cu}-\mathrm{NOTA}-\mathrm{Var} 7 \mathrm{in} \mathrm{BALB} / \mathrm{c}$ mice with orthotopic 4T1 breast cancer allografts 100

Table S3-2. Tissue uptake (mean \% ID/g $\pm \mathrm{SD}$ ) of ${ }^{64} \mathrm{Cu}-\mathrm{NOTA}-\operatorname{Var} 7$ in BALB/c mice with orthotopic 4T1 breast cancer allografts 101

Table S4-1. Tissue uptake (mean \%ID $\pm \mathrm{SD}$ ) of ${ }^{18} \mathrm{~F}-\mathrm{AlF}-\mathrm{NOTA}-\mathrm{WT}$ in BALB/c mice with orthotopic 4T1 breast cancer allografts 104

Table S4-2. Tissue uptake (mean \%ID/g $\pm \mathrm{SD}$ ) of ${ }^{18} \mathrm{~F}-\mathrm{AlF}-\mathrm{NOTA}-\mathrm{WT}$ in BALB/c 
mice with orthotopic $4 \mathrm{~T} 1$ breast cancer allografts 105

Table S5-1. Tissue uptake (mean \%ID $\pm \mathrm{SD}$ ) of ${ }^{18} \mathrm{~F}-\mathrm{AlF}-\mathrm{NOTA}-\mathrm{Var} 3$ in BALB/c mice with orthotopic $4 \mathrm{~T} 1$ breast cancer allografts 108

Table S5-2. Tissue uptake (mean $\%$ ID/g \pm SD) of ${ }^{18} \mathrm{~F}-\mathrm{AlF}-\mathrm{NOTA}-\mathrm{Var} 3$ in BALB/c mice with orthotopic $4 \mathrm{~T} 1$ breast cancer allografts

Table S6-1. Tissue uptake (mean \%ID $\pm \mathrm{SD}$ ) of ${ }^{18} \mathrm{~F}-\mathrm{AlF}-\mathrm{NOTA}-\mathrm{Var} 7 \mathrm{in} \mathrm{BALB} / \mathrm{c}$ mice with orthotopic $4 \mathrm{~T} 1$ breast cancer allografts 112

Table S6-2. Tissue uptake (mean \%ID/g $\pm \mathrm{SD}$ ) of ${ }^{18} \mathrm{~F}-\mathrm{AlF}-\mathrm{NOTA}-\operatorname{Var} 7$ in BALB/c mice with orthotopic $4 \mathrm{~T} 1$ breast cancer allografts 113 Table S7-1. Tissue uptake (mean \% ID $\pm \mathrm{SD}$ ) of ${ }^{64} \mathrm{Cu}-\mathrm{NO} 2 \mathrm{~A}-\mathrm{cys}$ TT in BALB/c mice with orthotopic $4 \mathrm{~T} 1$ breast cancer allografts 116

Table S7-2. Tissue uptake (mean \%ID/g $\pm \mathrm{SD}$ ) of ${ }^{64} \mathrm{Cu}-\mathrm{NO} 2 \mathrm{~A}-\mathrm{cys} \mathrm{WT}$ in BALB/c mice with orthotopic $4 \mathrm{~T} 1$ breast cancer allografts 117

Table S8-1. Tissue uptake (mean \%ID \pm SD) of ${ }^{64} \mathrm{Cu}-\mathrm{NO} 2 \mathrm{~A}-\mathrm{cys}$ Var7 in BALB/c mice with orthotopic $4 \mathrm{~T} 1$ breast cancer allografts

Table S8-2. Tissue uptake (mean \%ID/g $\pm \mathrm{SD}$ ) of ${ }^{64} \mathrm{Cu}-\mathrm{NO} 2 \mathrm{~A}-\mathrm{cys}$ Var7 in BALB/c mice with orthotopic $4 \mathrm{~T} 1$ breast cancer allografts

Table S9-1. Tissue uptake (mean \% ID \pm SD) of ${ }^{18} \mathrm{~F}-\mathrm{AlF}-\mathrm{NO} 2 \mathrm{~A}-\mathrm{cys}$ WT in BALB/c mice with orthotopic $4 \mathrm{~T} 1$ breast cancer allografts 124

Table S9-2. Tissue uptake (mean $\% \mathrm{ID} / \mathrm{g} \pm \mathrm{SD}$ ) of ${ }^{18} \mathrm{~F}-\mathrm{AlF}-\mathrm{NO} 2 \mathrm{~A}-\mathrm{cys}$ WT in BALB/c mice with orthotopic $4 \mathrm{~T} 1$ breast cancer allografts 125

Table S10-1. Tissue uptake (mean $\%$ ID \pm SD) of ${ }^{18} \mathrm{~F}$-AlF-NO2A-cys Var7 in BALB/c mice with orthotopic $4 \mathrm{~T} 1$ breast cancer allografts 128 
Table S10-2. Tissue uptake (mean $\% \mathrm{ID} / \mathrm{g} \pm \mathrm{SD}$ ) of ${ }^{18} \mathrm{~F}-\mathrm{AlF}-\mathrm{NO} 2 \mathrm{~A}-\mathrm{cys}$ Var7 in $\mathrm{BALB} / \mathrm{c}$ mice with orthotopic $4 \mathrm{~T} 1$ breast cancer allografts

Table S11-1. Tissue uptake (mean $\%$ ID $\pm \mathrm{SD}$ ) of ${ }^{64} \mathrm{Cu}-\mathrm{NO} 2 \mathrm{~A}-\mathrm{cys} V$ ar3 in BALB/c mice with orthotopic 4T1 breast cancer allografts 132 Table S11-2. Tissue uptake (mean \%ID/g \pm SD) of ${ }^{64} \mathrm{Cu}-\mathrm{NO} 2 \mathrm{~A}-\mathrm{cys} V \operatorname{ar} 3$ in BALB/c mice with orthotopic $4 \mathrm{~T} 1$ breast cancer allografts 133 Table S12-1. Tissue uptake (mean $\%$ ID \pm SD) of ${ }^{18}$ F-AlF-NO2A-cys Var3 in BALB/c mice with orthotopic $4 \mathrm{~T} 1$ breast cancer allografts 136

Table S12-2. Tissue uptake (mean $\% \mathrm{ID} / \mathrm{g} \pm \mathrm{SD}$ ) of ${ }^{18} \mathrm{~F}-\mathrm{AlF}-\mathrm{NO} 2 \mathrm{~A}-\mathrm{cys} V \mathrm{Var} 3$ in $\mathrm{BALB} / \mathrm{c}$ mice with orthotopic 4T1 breast cancer allografts 137

Table S13-1. Tissue uptake (mean $\%$ ID \pm SD) of ${ }^{64} \mathrm{Cu}-\mathrm{NO} 2 \mathrm{~A}-\mathrm{cys} V$ ar3 in nude mice with PC3 prostate cancer xenografts 140

Table S13-2. Tissue uptake (mean $\%$ ID/g $\pm \mathrm{SD}$ ) of ${ }^{64} \mathrm{Cu}-\mathrm{NO} 2 \mathrm{~A}$-cysVar3 in nude mice with PC3 prostate cancer xenografts.

Table S13-3. Tissue uptake (mean $\%$ ID $\pm \mathrm{SD}$ ) of ${ }^{64} \mathrm{Cu}-\mathrm{NO} 2 \mathrm{~A}$-cysVar3 in nude mice with LNCaP prostate cancer xenografts 142

Table S13-4. Tissue uptake (mean $\% \mathrm{ID} / \mathrm{g} \pm \mathrm{SD}$ ) of ${ }^{64} \mathrm{Cu}-\mathrm{NO} 2 \mathrm{~A}$-cysVar3 in nude mice with LNCaP prostate cancer xenografts. 143

Table S13-5. Tissue uptake (mean $\%$ ID $\pm \mathrm{SD}$ ) of ${ }^{64} \mathrm{Cu}-\mathrm{NO} 2 \mathrm{~A}-\mathrm{cys} V$ ar3 in C57B1/6 mice with orthotopic B16-F10 melanoma allografts 144

Table S13-6. Tissue uptake (mean \%ID/g $\pm \mathrm{SD}$ ) of ${ }^{64} \mathrm{Cu}-\mathrm{NO} 2 \mathrm{~A}-\mathrm{cys} V \operatorname{ar} 3$ in C57B1/6 mice with orthotopic B16-F10 melanoma allografts 145

Table S13-7. Tissue uptake (mean $\%$ ID \pm SD) of ${ }^{18}$ F-AlF-NO2A-cysVar3 in nude 
mice with PC3 prostate cancer xenografts 146

Table S13-8. Tissue uptake (mean $\% \mathrm{ID} / \mathrm{g} \pm \mathrm{SD}$ ) of ${ }^{18} \mathrm{~F}-\mathrm{AlF}-\mathrm{NO} 2 \mathrm{~A}-\mathrm{cys} V \mathrm{Var} 3$ in nude mice with PC3 prostate cancer xenografts

Table S13-9. Tissue uptake (mean $\%$ ID \pm SD) of ${ }^{18} \mathrm{~F}-\mathrm{AlF}-\mathrm{NO} 2 \mathrm{~A}$-cys Var3 in nude mice with $\mathrm{LNCaP}$ prostate cancer xenografts

Table S13-10. Tissue uptake (mean $\% \mathrm{ID} / \mathrm{g} \pm \mathrm{SD}$ ) of ${ }^{18} \mathrm{~F}-\mathrm{AlF}-\mathrm{NO} 2 \mathrm{~A}-\mathrm{cys} V$ ar3 in nude mice with $\mathrm{LNCaP}$ prostate cancer xenografts 149

Table S13-11. Tissue uptake (mean $\%$ ID \pm SD) of ${ }^{18}$ F-AlF-NO2A-cysVar3 in one group of C57B1/6 mice with orthotopic B16-F10 melanoma allografts and one group of BALB/c mice with orthotopic B16-F10 melanoma allografts

Table S13-12. Tissue uptake (mean $\% \mathrm{ID} / \mathrm{g} \pm \mathrm{SD}$ ) of ${ }^{18} \mathrm{~F}-\mathrm{AlF}-\mathrm{NO} 2 \mathrm{~A}-\mathrm{cys} V$ ar3 in one group of C57B1/6 mice with orthotopic B16-F10 melanoma allografts and one group of BALB/c mice with orthotopic B16-F10 melanoma allografts 151

Table S13-13. Tissue uptake (mean $\%$ ID \pm SD) of ${ }^{18} \mathrm{~F}-\mathrm{AlF}-\mathrm{NO} 2 \mathrm{~A}$-cysVar3 in nude mice with U87MG brain cancer xenografts 152

Table S13-14. Tissue uptake (mean $\%$ ID/g \pm SD) of ${ }^{18}$ F-AlF-NO2A-cysVar3 in nude mice with U87MG brain cancer xenografts

\section{CHAPTER 3}

Table 1. Biophysical and cytotoxicity parameters of pHLIP variants 182

Table S1. List of peptide sequences 197

Table S2. Molecular weights, times of elution, and corresponding percentages of acetonitrile 198 
Table S3. Positions of maxima of tryptophan fluorescence spectra and ratios of

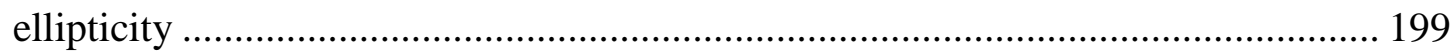

Table S4. Fluorescence intensities of tumor, tissues, and autofluorescence............. 200

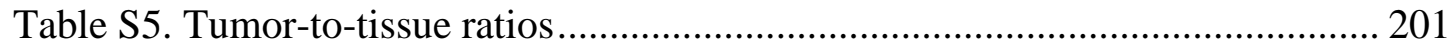

Table S6. Membrane-inserted populations of pHLIP variants at varying $\mathrm{pH}$........... 202 


\section{LIST OF FIGURES}

\section{CHAPTER 1}

Figure 1. pHLIP membrane interaction and insertion.......................................... 29

Figure 2. Mechanism of pHLIP insertion into the cellular membrane ..................... 30

Figure 3. Two uses for pHLIPs: tethering cargo to the cell surface or translocating

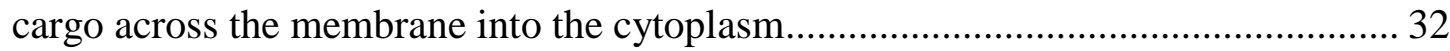

Figure 4. A postulated pathway for fusion and cellular uptake of pHLIP-coated

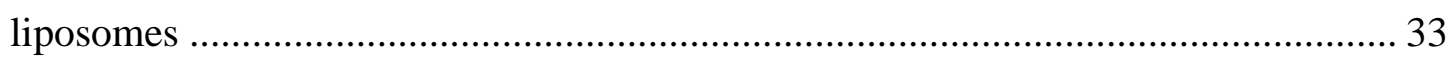

Figure I. The main features of sequences of the pHLIP peptide family .................... 35

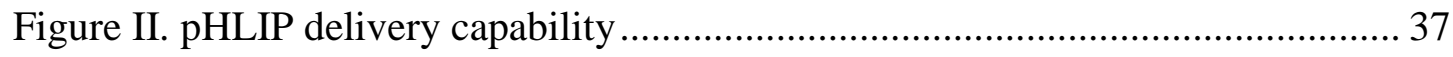

\section{CHAPTER 2}

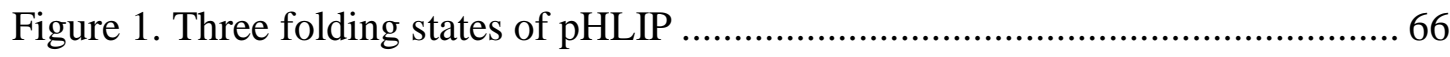

Figure 2. Chemical structures of the two chelators and sequences of the three peptides

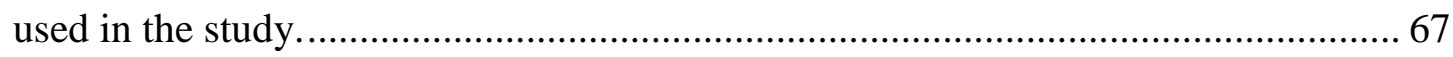

Figure 3. Coronal PET slices and ex vivo biodistributions ..................................... 68

Figure 4. $\mathrm{pH}$ dependent bilayer insertion of lead constructs................................6 69

Figure 5. PET slices and maximum intensity projections in various cancer models .. 70

Figure 6. Ex vivo tumor uptake of lead constructs in various cancer models ............ 71 
Figure 7. Comparison of uptake in PC3 and LNCaP tumors

Figure S1. PET maximum intensity projections for all tracers 83

Figure S2. Three state fluorescence of NO2A-cysVar3 and NOTA-Var3 84

Figure S3. State II and III fluorescence of cysteine-conjugated NO2A-pHLIP

constructs 85

Figure S4. $\mathrm{pH}$ dependent bilayer insertion of cysteine-conjugated constructs 86

Figure S5. Initial results from brain tumor uptake study 87

Figure S6. Relative autoradiography of LNCaP and PC3 tumors 88

Figure S7. Comparison of tumor uptake for PC3 and LNCaP tumors to previously reported results 89

Figure S8. Comparison of tumor-to-tissue ratios to previously reported results ........ 90

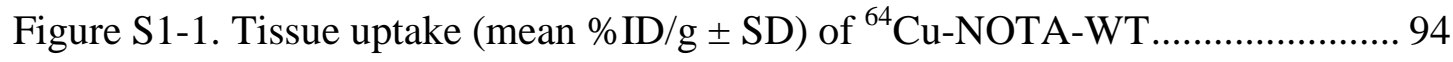

Figure S1-2. HPLC chromatogram of reconstituted ${ }^{64} \mathrm{Cu}-$ NOTA-WT injectate ........ 95

Figure S2-1. Tissue uptake (mean $\%$ ID/g \pm SD) of ${ }^{64} \mathrm{Cu}-\mathrm{NOTA}-\mathrm{Var} 3 \ldots \ldots \ldots \ldots \ldots \ldots . . . . . . . . . . .98$

Figure S2-2. HPLC chromatogram of reconstituted ${ }^{64} \mathrm{Cu}-\mathrm{NOTA}-\mathrm{Var} 3$ injectate....... 99

Figure S3-1. Tissue uptake (mean \%ID/g \pm SD) of ${ }^{64} \mathrm{Cu}-\mathrm{NOTA}-\operatorname{Var} 7 \ldots \ldots \ldots \ldots \ldots \ldots . . . . . . . . .102$

Figure S3-2. HPLC chromatogram of reconstituted ${ }^{64} \mathrm{Cu}-\mathrm{NOTA}-\mathrm{Var} 7$ injectate..... 103

Figure S4-1. Tissue uptake (mean $\%$ ID/g \pm SD) of ${ }^{18}$ F-AlF-NOTA-WT................ 106

Figure S4-2. HPLC chromatogram of reconstituted ${ }^{18}$ F-AlF-NOTA-WT injectate 107

Figure S5-1. Tissue uptake (mean $\%$ ID/g \pm SD) of ${ }^{18}$ F-AlF-NOTA-Var3 .............. 110

Figure S5-2. HPLC chromatogram of reconstituted ${ }^{18}$ F-AlF-NOTA-Var3 injectate

Figure S6-1. Tissue uptake (mean $\% \mathrm{ID} / \mathrm{g} \pm \mathrm{SD}$ ) of ${ }^{18} \mathrm{~F}-\mathrm{AlF}-\mathrm{NOTA}-\mathrm{Var} 7$. 114 
Figure S6-2. HPLC chromatogram of reconstituted ${ }^{18}$ F-AlF-NOTA-Var7 injectate 115

Figure S7-1. Tissue uptake (mean \%ID/g \pm SD) of ${ }^{64} \mathrm{Cu}-\mathrm{NO} 2 \mathrm{~A}$-cysWT................. 118

Figure S7-2. HPLC chromatogram of reconstituted ${ }^{64} \mathrm{Cu}-\mathrm{NO} 2 \mathrm{~A}-\mathrm{cys}$ WT injectate .119

Figure S8-1. Tissue uptake (mean \%ID/g \pm SD) of ${ }^{64} \mathrm{Cu}-\mathrm{NO} 2 \mathrm{~A}$-cysVar7 ............... 122

Figure S8-2. HPLC chromatogram of reconstituted ${ }^{64} \mathrm{Cu}-\mathrm{NO} 2 \mathrm{~A}-\mathrm{cys} V$ ar7 injectate 125

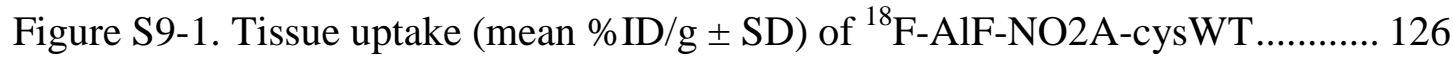

Figure S9-2. HPLC chromatogram of reconstituted ${ }^{18}$ F-AlF-NO2A-cysWT injectate

Figure S10-1. Tissue uptake (mean \%ID/g \pm SD) of ${ }^{18}$ F-AlF-NO2A-cysVar7 ........ 130

Figure S10-2. HPLC chromatogram of reconstituted ${ }^{18}$ F-AlF-NO2A-cysVar7 injectate

Figure S11-1. Tissue uptake (mean $\% \mathrm{ID} / \mathrm{g} \pm \mathrm{SD}$ ) of ${ }^{64} \mathrm{Cu}-\mathrm{NO} 2 \mathrm{~A}-\mathrm{cys}$ Var3 134

Figure S11-2. HPLC chromatogram of reconstituted ${ }^{64} \mathrm{Cu}-\mathrm{NO} 2 \mathrm{~A}-\mathrm{cys}$ Var3 injectate 135

Figure S12-1. Tissue uptake (mean \%ID/g $\pm \mathrm{SD}$ ) of ${ }^{18}$ F-AlF-NO2A-cysVar3 ........ 138

Figure S12-2. HPLC chromatogram of reconstituted ${ }^{18}$ F-AlF-NO2A-cysVar3 injectate 139

\section{CHAPTER 3}

Figure 1. Chemical structures and results of biophysical experiments with pHLIP bundles $183-4$ 
Figure 2. Ellipticity ratios of CD signals for all pHLIP variants and therapeutic indices

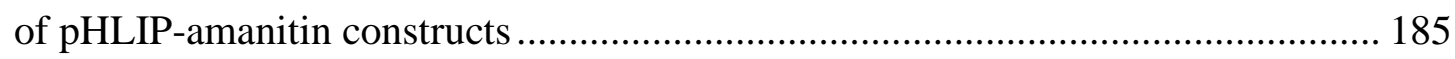

Figure 3. pH-dependent potency observed in in vitro experiments ........................ 186

Figure 4. Tumor fluorescence intensity and tumor-to-tissue intensity ratios from in

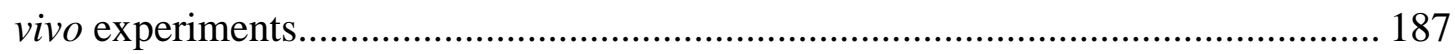

Figure S1. Three-state tryptophan fluorescence and circular dichroism for novel Var3-

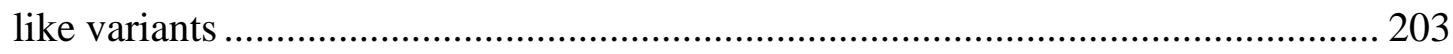

Figure S2. Cell viability data for pHLIP-amanitin constructs ............................. 204 


\title{
CHAPTER 1
}

Published in Trends in Biotechnology in July 2017

\section{Applications of pHLIP Technology for Cancer Imaging and Therapy}

\author{
Linden C. Wyatt ${ }^{1}$, Jason S. Lewis ${ }^{2}$, Oleg A. Andreev ${ }^{1}$, Yana K. Reshetnyak ${ }^{1}$, \\ Donald M. Engelman ${ }^{3 *}$
}

${ }^{1}$ Department of Physics, University of Rhode Island, Kingston, RI 02881, USA

${ }^{2}$ Department of Radiology, Memorial Sloan Kettering Cancer Center, 1275 York

Avenue, New York, NY 10065, USA; Program in Molecular Pharmacology,

Memorial Sloan Kettering Cancer Center, 1275 York Avenue, New York, NY 10065,

USA; Weill Cornell Medical College, 1300 York Avenue, New York, NY 10065,

USA

${ }^{3}$ Department of Molecular Biophysics and Biochemistry, Yale University, New

Haven, CT 06520, USA

*Correspondence: reshetnyak@uri.edu (Yana K. Reshetnyak) and

donald.engelman@yale.edu (Donald M. Engelman)

Keywords: Acidity, tumor targeting, imaging, drug delivery, nanotechnology 


\begin{abstract}
Acidity is a biomarker of cancer that is not subject to the blunting effects of clonal selection, effects that reduce the efficacy of other biomarker technologies, like antibody targeting. The $\mathrm{pH}$ (Low) Insertion Peptides (pHLIPs) provide new opportunities for targeting acidic tissues. Through the physical mechanism of membrane-associated folding, pHLIPs are triggered by the acidic microenvironment to insert and span the membranes of tumor cells. The pHLIP platform can be applied to imaging acidic tissues, delivering cell-permeable and impermeable molecules to the cytoplasm, and promoting the cellular uptake of nanoparticles. Since acidosis is a hallmark of tumor development, progression, and aggressiveness, the pHLIP technology may prove useful in targeting cancer cells and metastases for tumor diagnosis, imaging, and therapy.
\end{abstract}




\section{EXTRACELLULAR ACIDITY}

Acidity is a hallmark and predictor of tumor development and progression

Even in the presence of oxygen, cancer cells use the anaerobic pathway more than normal cells do, a phenomenon known as the Warburg effect [1-3]. Normally, cell metabolism occurs through a low rate of glycolysis followed by oxidation of pyruvate in the mitochondria, whereas anaerobic metabolism is associated with a high rate of glycolysis and lactic acid fermentation [4]. Protons are a byproduct of both pathways; however, when cells use glycolysis and lactic acid fermentation at a high rate, excess lactate and protons are produced. In order to maintain homeostasis, a cell must keep its intracellular $\mathrm{pH}$ at the physiological level, around $\mathrm{pH}$ 7.2-7.4. If the $\mathrm{pH}$ inside the cell drops, the cell will pump excess acidity into the extracellular space to maintain physiological $\mathrm{pH}$ levels in the cytoplasm. Additionally, cancer cells overexpress surface carbonic anhydrases (CAIX and CAXII), which catalyze the transformation of carbon dioxide and water into carbonic acid (bicarbonate and protons) [5-7]. Thus, excessive amounts of acid and protons accumulate in the extracellular space, especially in poorly perfused tumor regions, reducing the $\mathrm{pH}$ of the extracellular space and leading to tumor acidification $[8,9]$.

Not only is acidity a hallmark of tumor development, but acidity also facilitates tumor growth [10]. The acidification of the healthy tissue surrounding a tumor leads to tissue remodeling that allows for local invasion. Studies in which the $\mathrm{pH}$ of the surface of tumors was monitored showed that the regions of the highest tumor invasion (see Glossary) corresponded to areas of highest acidity [10]. Therefore, targeting the most 
acidic regions of tumors is especially beneficial because the most acidic regions are the most aggressive [11].

\section{Bulk extracellular $p H$ versus cancer cell surface $p H$}

Recent studies using a fluorophore with a $\mathrm{pH}$-dependent emission spectrum have shown that the $\mathrm{pH}$ in the vicinity of the plasma membrane of cancer cells is about 0.3-0.7 $\mathrm{pH}$ units lower than the bulk extracellular $\mathrm{pH}[12]$. In experiments in vitro, where the $\mathrm{pH}$ of the solution in which the cancer cells are growing (bulk $\mathrm{pH}$ ) is maintained at $\mathrm{pH} 7.4$, the cell surface $\mathrm{pH}$ for highly metastatic cells has been shown to be around $\mathrm{pH}$ 6.7. In three-dimensional tumor models, ex vivo mouse tumor tissue, and live animals, the cancer cell surface $\mathrm{pH}$ has been shown to be as low as $\mathrm{pH}$ 6.0. Thus, cancer cells have a "crown of acidity" near their cell surfaces. The $\mathrm{pH}$ becomes less acidic with distance from the cell surface and, therefore, the bulk extracellular $\mathrm{pH}$ can be relatively high, especially in well-perfused regions. However, the cell surface $\mathrm{pH}$ always remains low (acidic). The bulk extracellular $\mathrm{pH}$ correlates with perfusion, while the cell surface $\mathrm{pH}$ is expected to be less dependent on tumor tissue perfusion, and to be a predictive marker of tumor development and progression, since more aggressive tumor cells are more acidic.

\section{pHLIP TECHNOLOGY}

Peptides of the pHLIP family and their mechanism of action

The pH (Low) Insertion Peptide (pHLIP®, see Glossary) was derived from the C-helix of the protein bacteriorhodopsin, and originally was called the BRC peptide 
[13]. The salient feature of a pHLIP is its ability to sense the $\mathrm{pH}$ in the vicinity of the plasma membrane and to spontaneously form a helix and insert across the membrane when the extracellular environment is acidic (Figure 1) [14, 15]. Numerous modifications have been made to the primary sequence of pHLIPs to evaluate and tune the properties of the interaction of the pHLIP with the cell membrane [16]. These modifications include testing a pHLIP consisting entirely of D-amino acids against one containing entirely L-amino acids (no change was observed) [17]; truncating and reversing the wild-type (WT) pHLIP sequence, and in so doing introducing new pHLIP variants $[18,19]$; swapping some or all aspartic acid residues for glutamic acid residues $[16,20,21]$, positively-charged lysine residues $[18,22-30]$, or the protonatable non-standard amino acids such as $\gamma$-carboxyglutamic acid and $\alpha$ aminoadipic acid [31]; and the de novo design of a pHLIP variant [32].

Variation of the WT pHLIP sequence led to novel pHLIPs, such as Variant 3 (Var3), with significantly improved tumor targeting properties [18, 28, 33-35]. The overall features of the pHLIP peptide sequences are still present in all variants: a middle region interspersed with a combination of hydrophobic residues and residues that are negatively charged at physiological $\mathrm{pH}$ but become neutrally charged at low $\mathrm{pH}$, and hydrophilic flanking regions, with the membrane-inserting C-terminus (in most sequences) containing a few additional protonatable residues (Box 1) [9, 36-38]. Var3, in particular, has a truncated membrane-inserting end, which leads to its faster partitioning into the cell membrane to form a transmembrane helix. This variant exhibits the highest difference between the Gibbs free energies of its interaction with 
the membrane at low and high $\mathrm{pHs}$, which ensures $\mathrm{pH}$-dependent preferential targeting of the cancer cells [18].

The mechanism of action of all peptides in the pHLIP family is well understood: membrane-associated folding is triggered by the protonation and increase in hydrophobicity of the peptide when exposed to acidic environments (Figure 2) [15, 19, 22, 39, 40]. At physiological $\mathrm{pH}$, a population of pHLIPs mainly exists as an equilibrium between two states: a solvated state in which the pHLIP adopts a conformation with little secondary structure, and a state in which the pHLIP remains disordered and is adsorbed to the surface of the cell membrane [15]. At low $\mathrm{pH}$, an increased concentration of protons results in the protonation of negatively charged residues at key locations in the peptide. This protonation increases the overall hydrophobicity of the pHLIP, resulting in its folding to form a helix that partitions across the bilayer as a transmembrane helix. The inserted peptide leaves its $\mathrm{N}$ terminus in the extracellular space while the C-terminus is translocated across the membrane into the cytoplasm. The lipid composition of the cell membrane can contribute to modulation of the binding and insertion into the membrane of a pHLIP $[20,41,42]$.

It is important to distinguish between the pHLIP family, which consists of membrane-inserting peptides, and cell-penetrating peptides (CPPs) or amphipathic pore-forming peptides. CPPs and pore-forming peptides have entirely different mechanisms of action and different biological performance; they are not part of the pHLIP family. 


\section{Targeting extracellular acidity}

Targeting cancer cells by exploiting extracellular acidification should be compared with other targeting approaches, which are commonly based on the presence of growth factors or tumor antigens. A problem in targeting these kinds of specific features is that the cancer cell population, both between tumors and within an individual tumor, is extremely heterogeneous and very adaptable [43]. The potential for successfully treating tumors that do not express or exhibit limited expression of the targeted growth factor or antigen is low, and, even in treatment-responsive tumors, minority populations of cancer cells can undergo clonal selection and eventually regrow into a treatment-resistant phenotype. Subsequent treatments will either have limited efficacy or be entirely ineffective. Additionally, these types of targeting methods have the potential to severely damage healthy tissues where the growth factor or antigen might also be present. Therefore, targeting tumor acidity, a physical characteristic that is not only found across an entire tumor but is found in tumors of all sizes, including metastases, could have advantages over targeting methods based on characteristics that are found only in some tumors or some cancer cell populations [44, 45].

Acidity is produced not only by cancerous tissue, but also by any tissue that is experiencing hypoxia: the lack of oxygen in the tissue triggers cells to switch to a high rate of the anaerobic, glycolytic energy production pathway, consequently resulting in acidification. pHLIPs have been shown to target infections in lungs [46], inflammatory arthritis [22], and ischemic myocardium, a consequence of heart disease, and could be used for diagnosis and treatment thereof [26]. It is also possible that pHLIPs could be 
used as targeted treatment in ischemic strokes. In contrast to diseased tissue, healthy tissue is typically not associated with increased acidity except in the gastrointestinal tract and kidney, the pHs of which might be regulated by the implementation of a special diet or supplementary drinks if their acidity proves problematic.

In contrast to other $\mathrm{pH}$-sensitive agents that sense bulk $\mathrm{pH}$, members of the pHLIP family of peptides bind to cell surfaces at all $\mathrm{pH}$ values and therefore sense $\mathrm{pH}$ at the surfaces of cancer cells, where $\mathrm{pH}$ is the lowest, further accentuating the $\mathrm{pH}$ sensitivity of the peptides. The $\mathrm{pH}$-dependent behavior and inserted conformation of pHLIPs make them useful for delivering cargo inside cancer cells (by attaching the cargo to the inserting end of the peptides) or for tethering cargo to the cell membrane (by conjugating to the non-inserting end of the peptides) [9]. Additionally, small-angle X-ray scattering experiments have shown that pHLIPs do not form oligomers larger than tetramers, even at very high concentrations [47], a useful property for drugs to be used intravenously, as the local concentration at the injection site is generally much higher than the drug concentration when it reaches the target area, and it is crucial that the drug does not aggregate upon injection. These basic features give rise to a wide variety of possible applications in diagnostic imaging, fluorescence guided surgery, the intracellular delivery of small molecules like toxins and gene regulation agents, the delivery of proteins to the surfaces of cells, and the delivery and uptake enhancement of nanoparticles.

\section{APPLICATIONS OF pHLIP TECHNOLOGY}

Diagnostic nuclear imaging 
Positron emission tomography (PET) and single-photon emission computed tomography (SPECT) are imaging techniques that rely on the delivery of radioactive imaging agents to targeted tissue. One of the main challenges in PET/SPECT imaging is to obtain images of target tissue that contrast highly with the image background, motivating the use of a targeting agent. When a radionuclide is conjugated to the noninserting terminus of a pHLIP (Figure 3A), the resulting construct not only targets tumor tissue very well, but also remains securely in the tumor long enough to allow the excess, non-inserted construct to clear from normal tissues, resulting in higher contrast images $[17,23,25,29,33]$. Success has been seen using the radioisotopes ${ }^{18} \mathrm{~F}$ $(9 \pm 2 \% \mathrm{ID} / \mathrm{g}$ at $4 \mathrm{~h}$ p.i. $)$ and ${ }^{64} \mathrm{Cu}(19 \pm 2 \% \mathrm{ID} / \mathrm{g}$ at $24 \mathrm{~h}$ p.i. $)$ conjugated to $\operatorname{Var} 3$ [33]. These constructs are in the process of clinical translation as novel nuclear imaging markers of diseased tissue acidity.

Fluorescence-guided surgery and ex vivo imaging

Fluorescence-guided surgery utilizing pHLIP technology could ease the challenges of tumor resection, such as visualization of all cancerous lesions, including flat lesions, ultimately reducing the number of surgeries that result in positive margins. These features could improve surgery outcomes and reduce tumor recurrences. Excellent targeting and labeling by different fluorescent pHLIPs of primary tumors and sub-millimeter-sized metastatic lesions has been demonstrated on various tumor models, including transgenic breast, prostate, melanoma and pancreatic mice models [11, 24, 28, 34, 35, 48]. 
Excellent staining and visualization of urothelial tumors has been observed in excised human bladders using an ICG pHLIP imaging agent [48]. This imaging agent marked high-grade urothelial carcinomas, both muscle-invasive and non-muscleinvasive lesions, and, most importantly, flat lesions, which are the most difficult to identify and commonly necessitate further surgery. Carcinoma in situ was accurately diagnosed in eleven cases, whereas only four cases were identified using white light. The sensitivity and specificity of targeting by the ICG pHLIP imaging agent were found to be $97 \%$ and $100 \%$, respectively, excluding staining of necrotic and previously treated tissue.

Another application of fluorophore-labeled pHLIPs is the ex vivo staining and imaging of biopsy samples or tissue after surgical resections. Fluorophore-conjugated pHLIPs have shown utility in accurately identifying head and neck cancer, proving their potential to be useful tools for the early identification and evaluation of dysplasia and neoplasia $[49,50]$. In these studies, both the normal and suspect biopsy samples were stained ex vivo with Alexa Fluor647 pHLIP. Imaging was performed before and after staining, and the ratio of intensity increase of suspect tissue to normal tissue was calculated; a two- to six-fold increase in fluorescence intensity ratio was observed in cancerous tissue compared to normal tissue (with no ratio increase for the inflamed tissue).

The conjugation of the pH-sensing fluorescent dye SNARF to a pHLIP results in a compound that can measure $\mathrm{pH}$ at the surface of individual cells, which differs greatly from the more easily and commonly measured bulk pH [12]. Currently, approaches for cell surface $\mathrm{pH}$ mapping using SNARF-pHLIP in liquid and solid 
biopsy samples are being developed, which might result in an opportunity to get information about the metabolic status of tumors, ultimately aiding in the prediction of tumor aggressiveness and the tailoring of therapy. SNARF-pHLIP might also be used in longitudinal studies as a very useful tool in the investigation of the development and progression of diseases.

Intracellular delivery of drug-like and polar therapeutic cargo molecules

pHLIPs translocate their C-termini into the cytoplasms of tumor cells, allowing the intracellular delivery of therapeutic cargo molecules to treat primary tumor tissue as well as metastases. The ability of pHLIPs to target metastases is especially important: in cancer cases, the primary tumor is generally not the reason for poor prognoses; more often, it is tumor invasion, unseen by traditional diagnostic procedures, that proves fatal.

Extensive biophysical investigations, including kinetics and thermodynamics studies, have been conducted to determine the activation energy required for the membrane insertion/folding (as well as unfolding/exit) of pHLIPs [19-21, 39, 51]. These studies set the foundation for the development of novel approaches in drug delivery that are based on employing the energy of membrane-associated folding and the kinetically controlled $\mathrm{pH}$-triggered translocation of cargo molecules across the lipid bilayer of the cell membrane (Figure 3B). Cargoes can be linked to the Cterminus, for example, by including a cysteine in the pHLIP sequence and attaching the cargo via a disulfide that is cleaved in the cytoplasm, releasing the cargo. Following fundamental studies, numerous in vitro and in vivo investigations have 
demonstrated that pHLIPs can deliver several different payloads, such as fluorescent dyes, toxins, drugs, peptides, and peptide nucleic acids (PNAs) [14, 52-61]. The many molecules delivered into cells by pHLIPs can be conceptualized in two categories: drug-like, cell-permeable molecules; and polar, cell-impermeable cargoes. In the first case, pHLIPs might improve the pharmacokinetics and favorably alter the biodistribution of drugs, resulting in enhanced tumor accumulation. In the second case, pHLIPs, in addition to tumor targeting, can deliver polar molecules that cannot cross the plasma membrane via passive diffusion.

Additionally, it is possible to tune the intracellular delivery of cargo by altering the C-terminal sequence, by choices of the linker connecting cargo to a pHLIP, and/or by attaching modulator molecules [52-54]. Changing the efficiency of cargo translocation leads to a change in biological response: systematic studies in which polar toxins are conjugated to pHLIPs via cleavable linkers of different hydrophobicity show subtle changes in the insertion properties of the constructs and, therefore, in the resulting cytotoxicity. Thus, depending on cargo polarity, the translocation of cargo across cellular membranes could be optimized by the proper selection of pHLIPs, linkers and modulator molecules.

At low $\mathrm{pH}(\mathrm{pH}<6.5)$, $\mathrm{pHLIPs}$ promote delivery of cell-permeable therapeutic cargoes such as the tubulin-binding toxin monomethyl auristatin E (MMAE) and the DNA intercalator doxorubicin, and reduces the cellular entry of these cargoes into healthy tissue at normal physiological $\mathrm{pH}[57,58]$. pHLIP MMAE conjugates have cytotoxic effects at low $\mathrm{pH}$, inhibiting cell growth by more than $90 \%$, and targeted triple-negative breast cancer tumors in mice [58]. 
One of the most attractive therapeutic applications of pHLIPs is their ability to deliver polar molecules. Studies have been conducted using polar cyclic toxins from the death cap mushroom (Amanita phalloides): phalloidin, phallacidin and amanitin. Phalloidin and phallacidin bind to F-actin, while amanitin is an inhibitor of RNA polymerase II. All toxins, when conjugated to $\mathrm{pHLIPs}$, have shown a $\mathrm{pH}$-dependent ability to induce cell death $[52,53] . \alpha$-Amanitin is the most potent cargo studied so far, and, when conjugated to a pHLIP and exposed to cancer cells for less than two hours, it displayed a four- to five-fold higher antiproliferative effect at low $\mathrm{pH}(\mathrm{pH}$ 6.5) compared with physiological pH [54].

Also, pHLIPs have been proven to deliver peptides to interfere with the biological action of protein receptors or to disrupt mitochondrial cellular membranes $[59,60]$. PAR1 is a protein receptor that is overexpressed in many cancer cells. A PAR1 protein fragment was shown to be translocated across the cell membrane by a pHLIP, where it interacted with the intracellular domain of the PAR1 receptor, rendering the protein receptor inactive and inducing $\mathrm{pH}$-dependent cytotoxicity [59].

\section{Intracellular delivery of gene-regulation agents}

There is an expectation that future therapy (including cancer therapy) will be based on drugs that induce genetic interference in cancer cells. Efficiently modulating gene expression inside cancer cells and either reprogramming them to be normal cells or inducing apoptosis will be a significant breakthrough. Oncogenic microRNAs (oncomiRs) are miRNAs whose overexpression is associated with cancer 
development; thus, it is desirable to inhibit an oncomiR, which can be achieved using complementary synthetic RNA strands, such as PNAs [56, 62, 63].

The main obstacle to the successful use of PNA antimiRs is that they do not cross cell membranes. pHLIPs, however, have been shown to deliver PNAs to cancer cells by virtue of their inherent targeting capability and folding energy. pHLIPmediated intracellular delivery of PNAs targeting the miR-155 oncomiR was shown in a mouse model of large B-cell lymphoma [56]. Treatment of lymphadenopathic miR-155 mice with pHLIP anti-oncomiR PNA leads to tumor burden reduction and prevention of lymphocyte metastases.

Another approach to gene therapy involves the intracellular delivery of plasmid DNAs (pDNAs). In one study, a pDNA expressing growth factor-interfering RNA was attached to a dendrimer via electrostatic interaction, and the dendrimerpDNA complex was conjugated to a pHLIP. The study showed higher cellular uptake at low $\mathrm{pH}$ compared to normal $\mathrm{pH}$ and resulted in an $86 \%$ inhibition of the targeted growth factor [64].

\section{Targeting of therapeutic agents to cell surfaces}

Another interesting use for pHLIPs is the delivery of therapeutic agents to the extracellular surfaces of cells, which might selectively activate various signaling pathways. An example of this principle is shown in the use of tissue factor (TF), a protein that anchors to the plasma membrane and induces blood coagulation. However, targeting is crucial for the successful use of tissue factor to accomplish thrombotic occlusion; otherwise, harmful side effects would be common. The tissue 
factor protein naturally has a transmembrane domain that anchors it to cell surfaces. A study in which the transmembrane domain of the natural tissue factor was truncated and replaced with a pHLIP (resulting in tTF-pHLIP) showed targeting of the acidic tumor vasculature, and the tTF-pHLIP induced local blood coagulation. The administration of the tTF-pHLIP selectively induced thrombotic occlusion in tumorbearing mice, impairing tumor growth without any obvious side effects [65].

\section{Nanotechnology}

In the realm of nanotechnology, pHLIPs might find a variety of uses [66]. In nanotechnology applications, multiple pHLIPs decorate a single nanoparticle, which can range in size from a few nanometers to hundreds of nanometers, in contrast to the single peptide-cargo conjugates described above (Box 2). Nanocarriers decorated with pHLIPs are biocompatible, can target tumors, and demonstrate enhanced cellular uptake by cancer cells. Some pHLIP-coated nanoparticles that have been investigated are lipid, polymer, and metal-based nanomaterials [27, 67, 68].

In pHLIP-coated liposomes, pHLIPs promote lipid exchange and fusion between the lipid bilayer of the liposome and the cellular membrane [67]. Fusion with cellular or endosomal membranes allows the direct release of polar payloads into the cytoplasm, or the transfer of hydrophobic payloads into the membrane lipid bilayer (Figure 4) [67]. The principle of pHLIP-mediated liposomal fusion was used to deliver the hydrophobic peptide gramicidin A, a monovalent ion channel. Introducing pores into the targeted cell membrane disrupts ion balance, resulting in apoptosis [67]. This case demonstrates that pHLIPs might be used not only to improve liposome 
targeting over passive targeting methods, but also to enhance payload delivery into cells by promoting the fusion of the liposome with the cell membrane.

Gold nanoparticles have found various applications in medicine, including imaging, radiation therapy enhancement, and hyperthermia localization. The conjugation of pHLIP to gold nanoparticles has been shown to enhance cancer cell uptake by over $600 \%$ at low $\mathrm{pH}$ compared with cells treated with gold nanoparticles alone [27], and to promote the internalization of gold nanoparticles in human platelets [69]. Tumor targeting specificity was observed in studies of pHLIP-coated gold nanoclusters, ultimately resulting in the enhancement of radiation effects and increased cytotoxicity [70]. Additionally, pHLIP-coated hollow gold nanospheres (HAuNS) containing chlorin e6 (Ce6), a chemical used to amplify the response of tumor cells to photodynamic therapy, were developed. The HAuNS-pHLIP-Ce6, when irradiated with near-infrared light, treated tumors more effectively than hollow gold nanospheres alone because of the photosensitizer-enhanced hyperthermia due to $\mathrm{Ce} 6$ $[71,72]$.

\section{CONCLUDING REMARKS}

pHLIPs have been shown to target cancer tumor acidity. Targeting that exploits acidity, a physical characteristic ubiquitous to tumors, may have advantages when compared to other current approaches. Passive targeting, which relies on the abnormal vasculature present in large tumors, and active targeting, which relies on specific traits of cancer cells, have disadvantages due to the inconsistent presentation of passively-targeted traits, the heterogeneity of the cancer cell population, and the 
presentation of targeted traits in healthy cells. These problems can result in failure to target some cancer cells, the selection of resistant tumor cells, and, ultimately, ineffective diagnosis, surgery, and therapy due to relapse, the development of treatment-resistant cancer, and significant damage to healthy tissue.

Not only do pHLIPs target both primary tumor tissue and metastases well, but the characteristics of the insertion of pHLIPs into cancer cells may make them a useful tool for many applications (see Outstanding Questions). pHLIPs have been used to tether imaging agents to cell surfaces for diagnostic imaging and fluorescence guided surgery. Additionally, the energy of insertion of pHLIPs has been used to deliver many different types of cargoes into the cytoplasm: small molecules such as toxins, chemotherapeutic agents, and protein fragments; PNA and pDNA for gene therapy; and nanomaterials such as liposomes, gold nanoparticles for therapy and imaging, and drug-laden porous nanoparticles. The pHLIP family of peptides presents novel opportunities in cancer diagnosis and targeted therapy due to the customizable nature of the peptides for uses in a wide variety of applications.

\section{ACKNOWLEDGEMENTS}

The research reported in this publication was supported in part by the National Institute of General Medical Sciences of the National Institutes of Health under award number R01GM073857 to OAA, YKR, and DME, and by the National Cancer Institute of the National Institutes of Health under award number R01CA138468 to JSL and award number P30CA08748 (MSK CCSG). The authors thank all the members of the Andreev, Engelman, Lewis, and Reshetnyak laboratories for their essential contributions to the pHLIP project. 


\section{OUTSTANDING QUESTIONS}

- How can pHLIP® be more effectively used in therapeutic applications? One of the greatest strengths of pHLIP® is its ability to deliver polar and moderately hydrophobic cargo molecules across the cellular membrane directly into the cytoplasm, avoiding the endocytotic pathway and reducing drug resistance. Optimized pHLIP® drug conjugates could be extremely useful in therapeutic applications, especially for the treatment of metastatic cancers and cancers which are difficult to treat, such as triple-negative breast cancers, pancreatic cancer, infiltrating and diffusive glioblastomas, and potentially many others.

- How can pHLIP® and/or the link between peptide and cargo be optimized to exclusively target cancer cells in order to achieve intracellular delivery of highly toxic cargoes? Off-target delivery of highly toxic chemotherapeutic agents (e.g., amanitin) has serious consequences and should be reduced even if the number of molecules delivered to the target tissue is consequently reduced. Typically, the delivery of just a few very potent drug molecules is enough to induce a cytotoxic effect.

- How can pHLIP® and/or the link between peptide and cargo be optimized for the efficient intracellular delivery of large quantities of less toxic cargoes? For some cargoes, like those which alter gene expression (e.g., PNA), the cargo itself is very specific to its target within cancer cells, and off-target delivery does not pose significant problems. At the same time, these types of cargoes need to be delivered in larger quantities to induce a noticeable biological effect. 


\section{GLOSSARY}

Amphipathic pore-forming peptides: Peptides possessing both hydrophobic and hydrophilic regions that insert and assemble to form pores in the cellular membrane Cell-penetrating peptide (CPP): A peptide, typically with a strong positive charge, that binds to the phospholipids of the cell membrane and is taken up by the cell Drug-like molecule: A molecule that exhibits properties similar to those of established drugs; for example, per Lipinski's Rule of Five: no more than five hydrogen bond donors, no more than ten hydrogen bond acceptors, molecular weight below $500 \mathrm{Da}$, and an octanol-water partition coefficient $\log P \leq 5$

F-actin: Filaments that are part of the cell cytoskeleton and contribute to structural stability and cell movement

ICG (indocyanine green): An FDA-approved fluorescent dye used to mark blood vessels in angiography and perfusion diagnostics, but does not target the tumor itself MicroRNA: Small molecules of RNA that play a functional role in gene expression by blocking messenger RNA translation

Non-standard amino acids: Amino acids that are not encoded in the human genome PAR1 (proteinase-activated receptor 1): A member of the large family of G proteincoupled receptors that plays a functional role in the blood coagulation process

Passive diffusion: The ability of molecules to cross cell membranes on their own

Passive targeting methods: Targeting methods that rely on naturally-occurring biological characteristics, such as non-intact tumor vasculature, to induce the localization of cargo within a tumor 
Peptide nucleic acid (PNA): Artificial DNA- or RNA-like molecule with a peptide backbone that forms a sequence-specific base-paired complex with DNA or RNA pHLIP®: The $\mathrm{pH}$ (low) insertion peptide; pHLIP is a registered trademark Positive margin: The condition that exists after surgical resection when the surgery was not successful in removing all cancerous tissue

RNA polymerase II: A key enzyme that catalyzes transcription

SNARF (seminaphtharhodafluor): A fluorescent dye with two pH-dependent emission peaks, making possible the use of SNARF to measure $\mathrm{pH}$ through spectrography or ratiometric imaging

Tissue factor (TF): Protein that is exposed to the blood upon tissue damage, triggering blood clot formation

Tubulin: A family of proteins that polymerize into microtubules, which are part of the cytoskeleton and contribute to structural stability, cytoplasmic transport, cell movement, and cell division

Transmembrane helix: The formation by a polypeptide of a helix that spans the lipid bilayer of a cellular membrane

Triple-negative breast cancer (TNBC): Any breast cancer that does not express the estrogen receptor, progesterone receptor, and epidermal growth factor receptor 2 (HER2/neu), making TNBC more difficult to treat due to the reliance of typical chemotherapies on the expression of one or more of these receptors

Tumor invasion: The spread of cancer cells (metastasis) from the primary tumor site to adjacent tissue or blood 


\section{REFERENCES}

1 Warburg, O., et al. (1924) Über den Stoffwechsel der Tumoren. Biochem Z 152, $319-344$

2 Warburg, O., et al. (1927) The Metabolism of Tumors in the Body. J Gen Physiol $8,519-530$

3 Warburg, O. (1956) On the origin of cancer cells. Science 123, 309-314

4 Krebs, H.A. (1972) The Pasteur effect and the relations between respiration and fermentation. Essays Biochem 8, 1-34

5 Wykoff, C.C., et al. (2000) Hypoxia-inducible expression of tumor-associated carbonic anhydrases. Cancer Res 60, 7075-7083

6 Potter, C.P. and Harris, A.L. (2003) Diagnostic, prognostic and therapeutic implications of carbonic anhydrases in cancer. Br J Cancer 89, 2-7

7 Swietach, P., et al. (2009) The role of carbonic anhydrase 9 in regulating extracellular and intracellular ph in three-dimensional tumor cell growths. $J$ Biol Chem 284, 20299-20310

8 Gillies, R.J., et al. (2012) Evolutionary dynamics of carcinogenesis and why targeted therapy does not work. Nat Rev Cancer 12, 487-493

9 Andreev, O.A., et al. (2014) Targeting diseased tissues by pHLIP insertion at low cell surface pH. Front Physiol 5, 97

10 Estrella, V., et al. (2013) Acidity generated by the tumor microenvironment drives local invasion. Cancer Res 73, 1524-1535

11 Tapmeier, T.T., et al. (2015) The pH low insertion peptide pHLIP Variant 3 as a novel marker of acidic malignant lesions. Proc Natl Acad Sci USA 112, 9710-9715 
12 Anderson, M., et al. (2016) Probe for the measurement of cell surface $\mathrm{pH}$ in vivo and ex vivo. Proc Natl Acad Sci USA 113, 8177-8181

13 Hunt, J.F., et al. (1997) Spontaneous, pH-dependent membrane insertion of a transbilayer alpha-helix. Biochemistry 36, 15177-15192

14 Reshetnyak, Y.K., et al. (2006) Translocation of molecules into cells by pHdependent insertion of a transmembrane helix. Proc Natl Acad Sci USA 103, 64606465

15 Reshetnyak, Y.K., et al. (2007) A Monomeric Membrane Peptide that Lives in Three Worlds: In Solution, Attached to, and Inserted across Lipid Bilayers. Biophys J 93, 2363-2372

16 Musial-Siwek, M., et al. (2010) Tuning the insertion properties of pHLIP. Biochim Biophys Acta 1798, 1041-1046

17 Macholl, S., et al. (2012) In vivo pH imaging with (99m)Tc-pHLIP. Mol Imaging Biol 14, 725-734

18 Weerakkody, D., et al. (2013) Family of pH (low) insertion peptides for tumor targeting. Proc Natl Acad Sci USA 110, 5834-5839

19 Karabadzhak, A.G., et al. (2012) Modulation of the pHLIP transmembrane helix insertion pathway. Biophys $J$ 102, 1846-1855

20 Barrera, F.N., et al. (2012) Membrane physical properties influence transmembrane helix formation. Proc Natl Acad Sci USA 109, 14422-14427

21 Fendos, J., et al. (2013) Aspartate Embedding Depth Affects pHLIP's Insertion pKa. Biochemistry 52, 4595-4604 
22 Andreev, O.A., et al. (2007) Mechanism and uses of a membrane peptide that targets tumors and other acidic tissues in vivo. Proc Natl Acad Sci USA 104, 78937898

23 Vavere, A.L., et al. (2009) A Novel Technology for the Imaging of Acidic Prostate Tumors by Positron Emission Tomography. Cancer Res 69, 4510-4516

24 Reshetnyak, Y.K., et al. (2011) Measuring tumor aggressiveness and targeting metastatic lesions with fluorescent pHLIP. Mol Imaging Biol 13, 1146-1156

25 Daumar, P., et al. (2012) Efficient (18)F-labeling of large 37-amino-acid pHLIP peptide analogues and their biological evaluation. Bioconjugate Chem 23, 15571566

26 Sosunov, E.A., et al. (2013) pH (low) insertion peptide (pHLIP) targets ischemic myocardium. Proc Natl Acad Sci USA 110, 82-86

27 Yao, L., et al. (2013) pHLIP peptide targets nanogold particles to tumors. Proc Natl Acad Sci USA 110, 465-470

28 Cruz-Monserrate, Z., et al. (2014) Targeting Pancreatic Ductal Adenocarcinoma Acidic Microenvironment. Sci Rep 4, 4410

29 Viola-Villegas, N.T., et al. (2014) Understanding the pharmacological properties of a metabolic PET tracer in prostate cancer. Proc Natl Acad Sci USA 111, 72547259

30 Kimbrough, C.W., et al. (2015) Targeting Acidity in Pancreatic Adenocarcinoma: Multispectral Optoacoustic Tomography Detects pH-low Insertion Peptide Probes in vivo. Clin Cancer Res 21, 4576-4585 
31 Onyango, J.O., et al. (2015) Noncanonical amino acids to improve the $\mathrm{pH}$ response of pHLIP insertion at tumor acidity. Angew Chem Int Edit 54, 3658-3663

32 Nguyen, V.P., et al. (2015) A novel soluble peptide with pH-responsive membrane insertion. Biochemistry 54, 6567-6575

33 Demoin, D.W., et al. (2016) PET imaging of extracellular pH in tumors with ${ }^{64} \mathrm{Cu}$ and ${ }^{18}$ F-labeled pHLIP peptides: A structure-activity optimization study. Bioconjugate Chem 27, 2014-2023

34 Adochite, R.C., et al. (2014) Targeting breast tumors with pH (low) insertion peptides. Mol Pharm 11, 2896-2905

35 Adochite, R.C., et al. (2016) Comparative study of tumor targeting and biodistribution of $\mathrm{pH}$ (low) insertion peptides (pHLIP peptides) conjugated with different fluorescent dyes. Mol Imaging Biol 113, 11829-11834

36 Andreev, O.A., et al. (2009) Targeting acidic diseased tissue: New technology based on use of the pH (Low) Insertion Peptide (pHLIP). Chim Oggi Chem Today $27,34-37$

37 Andreev, O.A., et al. (2010) pH-sensitive membrane peptides (pHLIPs) as a novel class of delivery agents. Mol Membr Biol 27, 341-352

38 Deacon, J., et al. (2015) Targeting acidity in diseased tissues: mechanism and applications of the membrane-inserting peptide, pHLIP. Arch Biochem Biophys 0, $40-48$

39 Reshetnyak, Y.K., et al. (2008) Energetics of peptide (pHLIP) binding to and folding across a lipid bilayer membrane. Proc Natl Acad Sci USA 105, 1534015345 
40 Zoonens, M., et al. (2008) Bilayer Interactions of pHLIP, a Peptide that Can Deliver Drugs and Target Tumors. Biophys J 95, 225-235

41 Kyrychenko, A., et al. (2015) Lipid Headgroups Modulate Membrane Insertion of pHLIP Peptide. Biophys J 108, 791-794

42 Scott, H.L., et al. (2015) The negative charge of the membrane has opposite effects on the membrane entry and exit of $\mathrm{pH}$-low insertion peptide. Biochemistry $54,1709-1712$

43 Marusyk, A. and Polyak, K. (2010) Tumor heterogeneity: causes and consequences. Biochim Biophys Acta 1805, 105

44 Gerweck, L.E. and Seetharaman, K. (1996) Cellular pH Gradient in Tumor versus Normal Tissue: Potential Exploitation for the Treatment of Cancer. Cancer Res 56, 1194-1198

45 Lloyd, M.C., et al. (2016) Darwinian Dynamics of Intratumoral Heterogeneity: Not Solely Random Mutations but Also Variable Environmental Selection Forces. Cancer Res 76, 3136-3144

$46 \mathrm{Li}$, N., et al. (2013) Peptide targeting and imaging of damaged lung tissue in influenza-infected mice. Future Microbiol 8, 257-269

47 Narayanan, T., et al. (2016) pHLIP(R) Peptide Interaction With Membrane Monitored by SAXS. J Phys Chem B 120, 11484-11491

48 Golijanin, J., et al. (2016) Targeted imaging of urothelium carcinoma in human bladders by an ICG pHLIP peptide ex vivo. Proc Natl Acad Sci USA 113, 1182911834 
49 Loja, M.N., et al. (2013) Optical Molecular Imaging Detects Changes in Extracellular $\mathrm{pH}$ with the Development of Head and Neck Cancer. Int J Cancer $132,1613-1623$

50 Luo, Z., et al. (2014) Widefield optical imaging of changes in uptake of glucose and tissue extracellular $\mathrm{pH}$ in head and neck cancer. Cancer Prev Res 7, 10351044

51 Barrera, F.N., et al. (2011) Roles of carboxyl groups in the transmembrane insertion of peptides. J Mol Biol 413, 359-371

52 An, M., et al. (2010) pH-(low)-insertion-peptide (pHLIP) translocation of membrane impermeable phalloidin toxin inhibits cancer cell proliferation. Proc Natl Acad Sci USA 107, 20246-20250

53 Wijesinghe, D., et al. (2011) Tuning a polar molecule for selective cytoplasmic delivery by a pH (Low) Insertion Peptide. Biochemistry 50, 10215-10222

54 Moshnikova, A., et al. (2013) Anti-proliferative effect of pHLIP-amanitin. Biochemistry 52, 1171-1178

55 Karabadzhak, A.G., et al. (2014) pHLIP-FIRE, a cell insertion-triggered fluorescent probe for imaging tumors demonstrates targeted cargo delivery in vivo. ACS Chem Biol 9, 2545-2553

56 Cheng, C.J., et al. (2015) MicroRNA silencing for cancer therapy targeted to the tumor microenvironment. Nature 518, 107-110

57 Song, Q., et al. (2015) A smart tumor targeting peptide-drug conjugate, pHLIPSS-DOX: synthesis and cellular uptake on MCF-7 and MCF-7/Adr cells. Drug Deliv 23, 1734-1746 
58 Burns, K.E., et al. (2015) Inhibition of cancer cell proliferation and breast tumor targeting of pHLIP-monomethyl auristatin E conjugates. Mol Pharm 12, 12501258

59 Burns, K.E. and Thévenin, D. (2015) Down-regulation of PAR1 activity with a pHLIP-based allosteric antagonist induces cancer cell death. Biochem J 472, 287295

60 Burns, K.E., et al. (2016) pH-selective cytotoxicity of pHLIP-antimicrobial peptide conjugates. Sci Rep 6, 28465

61 Thévenin, D., et al. (2009) pHLIP-mediated Translocation of Membrane Impermeable Molecules into Cells. Chemistry \& Biology 16, 754-762

62 Adams, B.D., et al. (2015) The Inescapable Influence of Noncoding RNAs in Cancer. Cancer Res 75, 5206-5210

63 Svoronos, A.A., et al. (2016) OncomiR or Tumor Suppressor? The Duplicity of MicroRNAs in Cancer. Cancer Res 76, 3666-3670

64 Han, L., et al. (2013) pH-Controlled Delivery of Nanoparticles into Tumor Cells. Adv Healthc Mater 2, 1435-1439

$65 \mathrm{Li}, \mathrm{S} .$, et al. (2015) pHLIP-mediated targeting of truncated tissue factor to tumor vessels causes vascular occlusion and impairs tumor growth. Oncotarget 6, 2352323532

66 Arachchige, M.C.M., et al. (2015) Advanced targeted nanomedicine. J Biotechnol 202, 88-97

67 Yao, L., et al. (2013) pHLIP(®)-Mediated Delivery of PEGylated Liposomes to Cancer Cells. J Control Release 167, 228-237 
68 Du, J.Z., et al. (2014) Tumor extracellular acidity-activated nanoparticles as drug delivery systems for enhanced cancer therapy. Biotechnol Adv 32, 789-803

69 Davies, A., et al. (2012) pH-controlled delivery of luminescent europium coated nanoparticles into platelets. Proc Natl Acad Sci USA 109, 1862-1867

70 Antosh, M.P., et al. (2015) Enhancement of radiation effect on cancer cells by gold-pHLIP. Proc Natl Acad Sci USA 112, 5372-5376

$71 \mathrm{Yu}$, M., et al. (2015) Photosensitizer-Loaded pH-Responsive Hollow Gold Nanospheres for Single Light-Induced Photothermal/Photodynamic Therapy. ACS Appl Mater Interfaces 7, 17592-17597

$72 \mathrm{Yu}, \mathrm{M}$., et al. (2016) A pH-Driven and photoresponsive nanocarrier: Remotelycontrolled by near-infrared light for stepwise antitumor treatment. Biomaterials 79, $25-35$ 


\section{FIGURES}

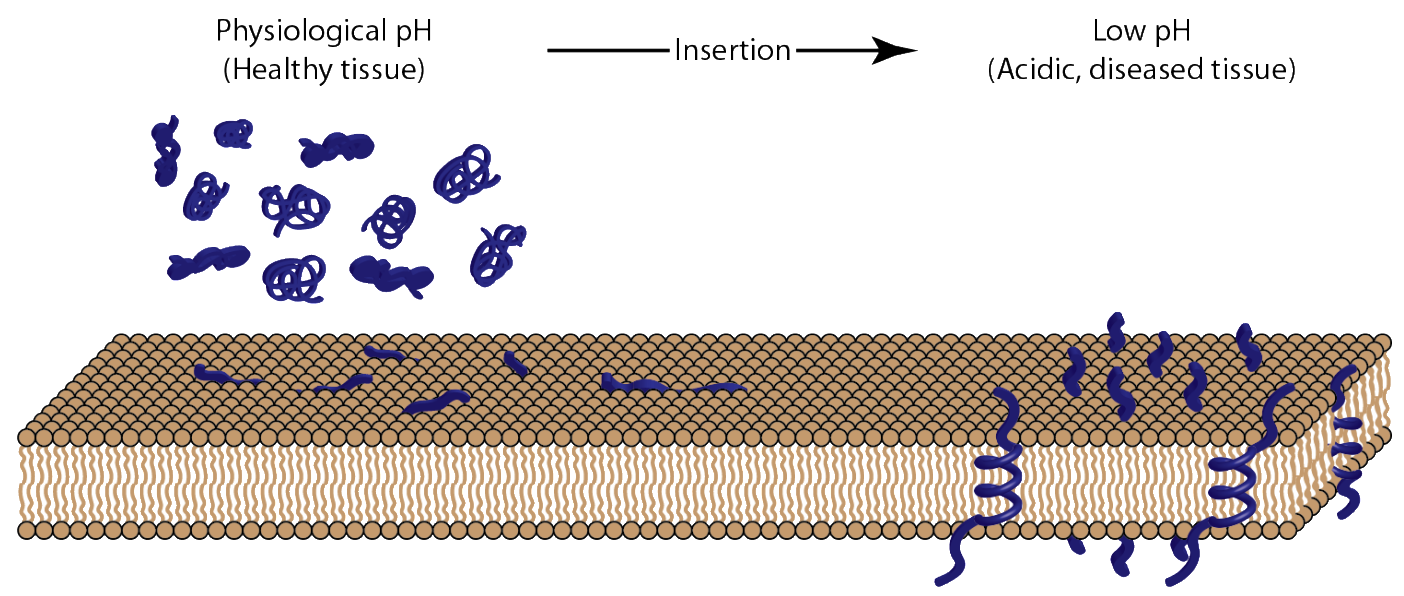

Figure 1. pHLIP membrane interaction and insertion. The peptides of the pHLIP

family (blue) exist in equilibrium between solvated and membrane-adsorbed

conformation at the normal extracellular $\mathrm{pH}$ found in healthy tissue, whereas peptides

insert across cellular membrane at the low extracellular $\mathrm{pH}$ found in acidic, diseased tissue. 


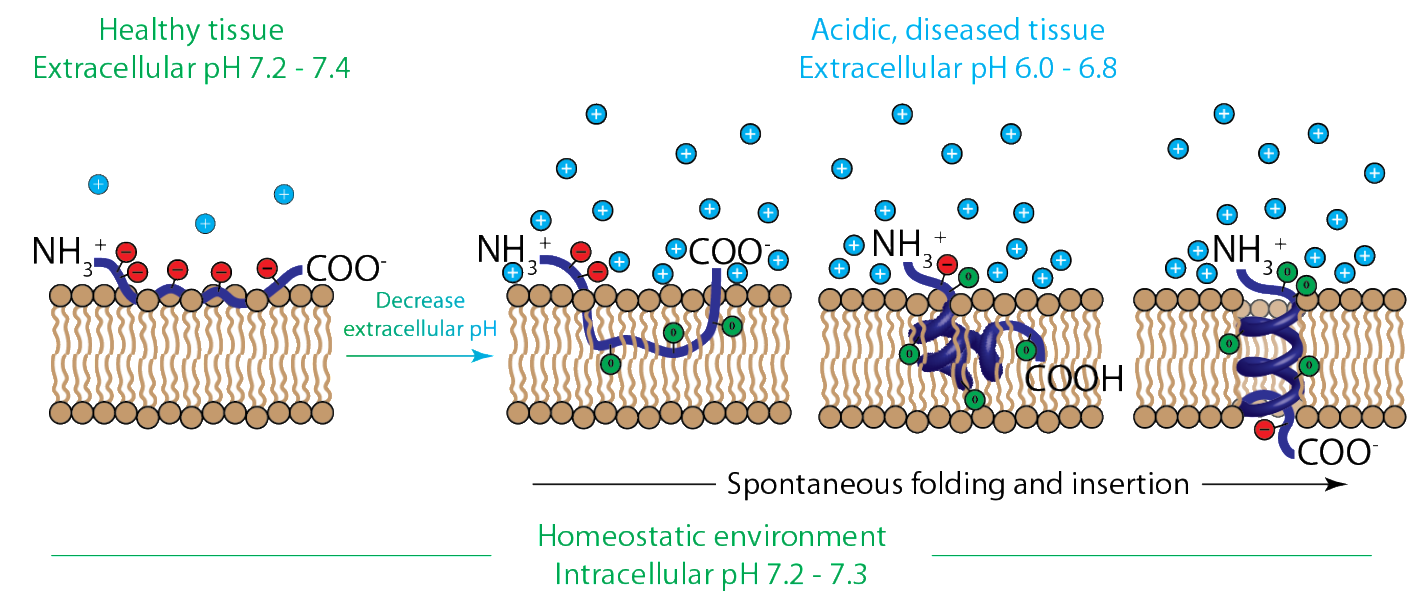

Figure 2. Mechanism of pHLIP insertion into the cellular membrane. When the pHLIP (blue) encounters healthy tissue where the extracellular $\mathrm{pH}$ is around $\mathrm{pH} 7.4$, the protonatable residues of the pHLIP (red circles) remain deprotonated and negatively charged, and the peptide resides at or near the hydrophilic surface of the cellular membrane. Weakly bound to the membrane, the pHLIP is washed from the membrane via normal perfusion and continues to circulate through the body. Cancer cells, however, produce excess acidity as a consequence of their malfunctioning metabolisms and overexpression of certain surface proteins, and pump these acidic byproducts out of the cell interior in order to maintain comfortable conditions inside the cell, resulting in the acidification of tumor tissue. When the pHLIP encounters tumor tissue, it senses the low extracellular $\mathrm{pH}$ at the cancer cell surface (i.e., the concentration of protons (cyan circles) at the surface of the cellular membrane is high), and the protonatable residues and negatively charged C-terminal carboxyl group of the pHLIP become neutrally charged (green circles). The protonation leads to an increase in the overall hydrophobicity of the pHLIP, increasing the affinity of the peptide to the hydrophobic core of the cellular membrane and triggering the pHLIP to 
spontaneously fold into a helix and insert across the membrane, resulting in the formation of a transmembrane helix. When the C-terminal protonatable residue and carboxyl group are then exposed to the normal intracellular $\mathrm{pH}$ of the cell, they are deprotonated, again becoming negatively charged, and anchor the pHLIP in the membrane. 

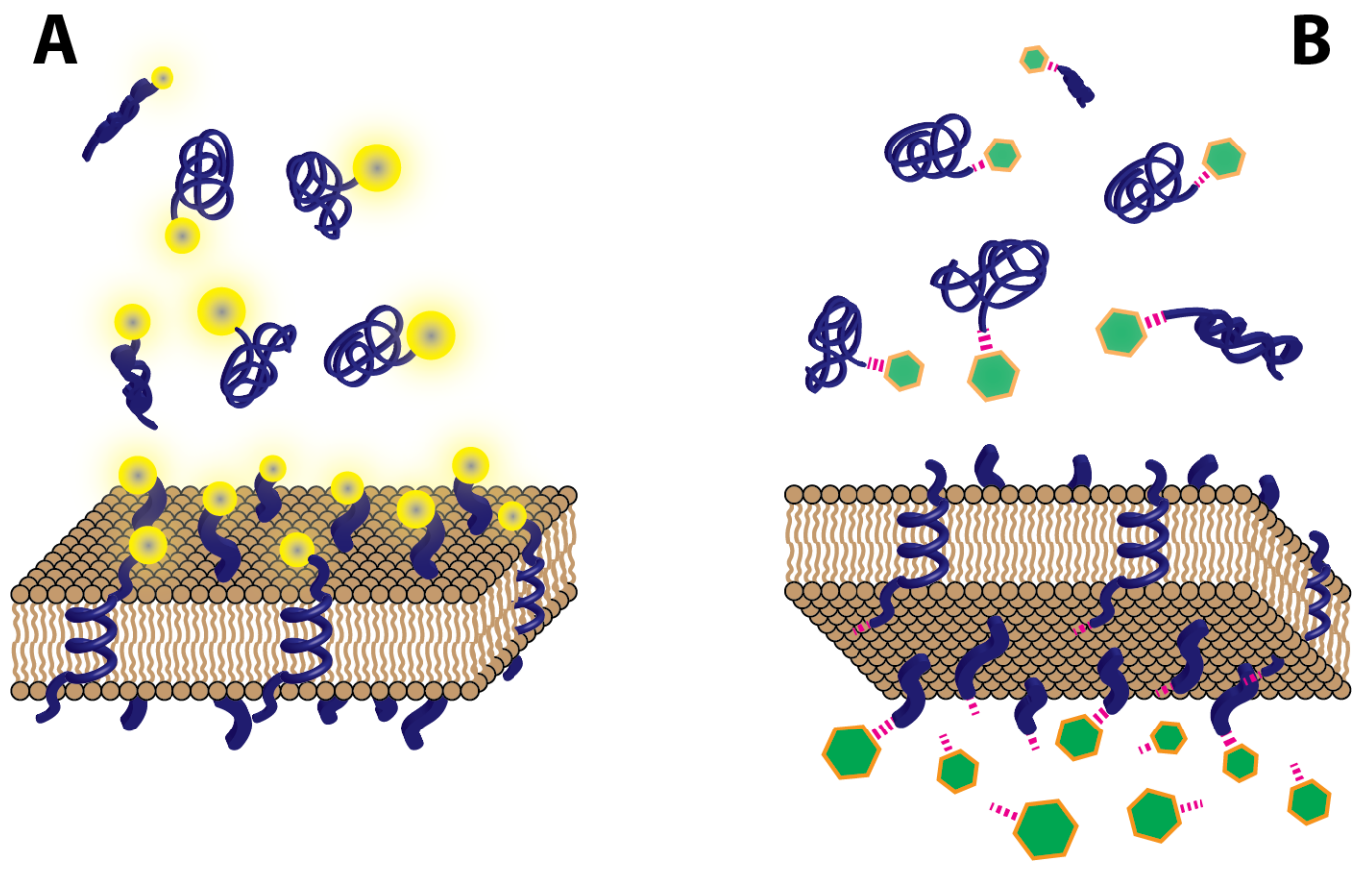

Figure 3. Two uses for pHLIPs: tethering cargo to the cell surface or translocating cargo across the membrane into the cytoplasm. A pHLIP (blue) can be used to target and tether cargo molecules to the surfaces of cells in low $\mathrm{pH}$ environments (A). The cargo could be an optical marker, a PET or SPECT imaging agent, or an antigen or protein delivered to induce certain cellular processes. A pHLIP can also be used for the intracellular delivery of payloads, translocating cargo (green) across the membranes of cells with low extracellular $\mathrm{pH}$, such as those cells found in acidic, diseased tissue $(\mathbf{B})$. These payloads are conjugated to the membrane-inserting end of the pHLIP, typically via a cleavable link (magenta), and could include toxins, chemotherapeutic agents, or agents to alter gene expression. 

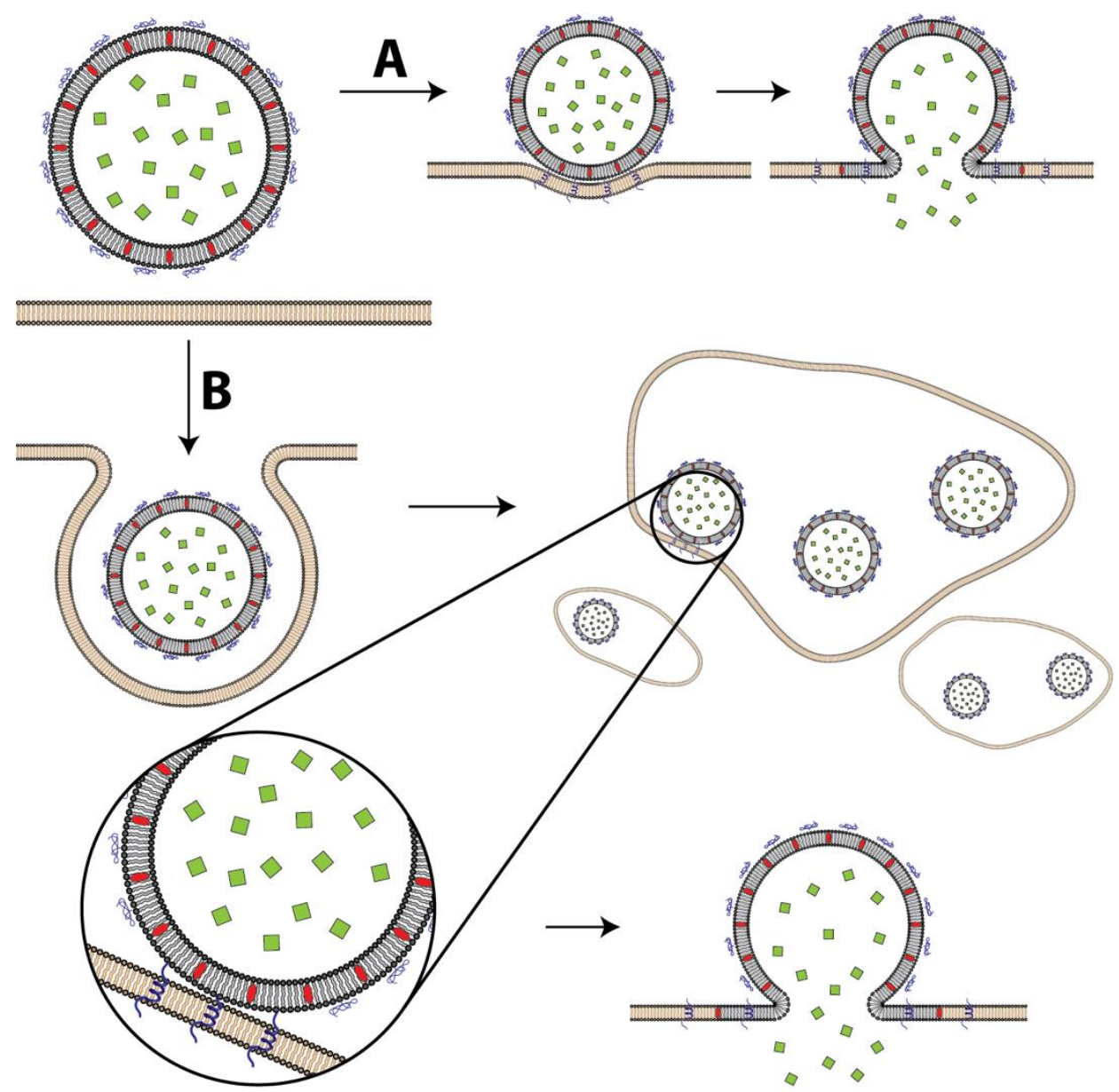

Figure 4. A postulated pathway for fusion and cellular uptake of pHLIP-coated

liposomes. pHLIP-coated liposomes interact with the membrane and promote either direct fusion with the lipid bilayer of the membrane (A), or uptake via the endocytotic pathway (B), followed by fusion with the endosomal membrane. Both uptake mechanisms result in the cytoplasmic delivery of encapsulated polar cargo (green), or delivery of lipid membrane-incorporated hydrophobic payloads to the membrane (red). Schematic is not to scale. 


\section{BOXES}

Box 1. Peptides of the pHLIP family share the same features in their primary sequences (Figure I) and exhibit the same mechanism of action (Figure 2). These shared characteristics include: i) an N-terminal region (flanking sequence 1) that varies from 3 to 20 residues and consists mainly of polar amino acids that contribute to the overall solubility of the peptide and are used for conjugation with cargo destined for the extracellular space; ii) a middle region (transmembrane sequence) that varies from 15 to 25 residues and consists mainly of hydrophobic residues, but also includes amino acids that are negatively charged at physiological $\mathrm{pH}$ but become neutrally charged at low $\mathrm{pH}$ due to protonation; and iii) a $\mathrm{C}$-terminal region (flanking sequence 2) that varies from 0 to 10 residues and may contain a few additional protonatable residues, as well as residues for conjugation with cargo that will be delivered across the cellular membrane to the cytoplasm. Single cysteine and/or lysine residue can be used for conjugation with cargo molecules and can be introduced into one or both flanking sequences.

These features are reflected in the sequences of the wild-type (WT) and the Variant 3 (Var3) pHLIPs, seen below, where protonatable residues are shown in red and the putative transmembrane region is underlined:

WT: AEQNPIY WARYADWLFTTPLLLLDLALLVDAD EGT

Var3: ADDQN PWRAYLDLLFPTDTLLLDLLW 


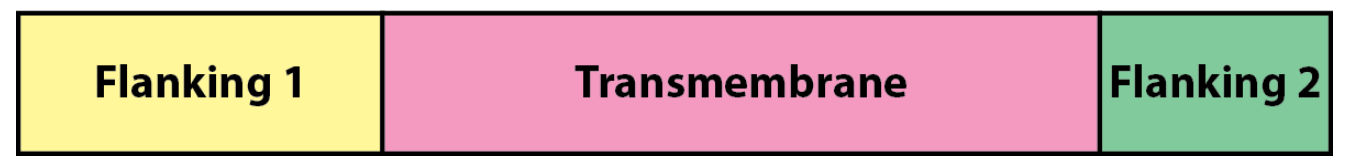

Figure I. The main features of sequences of the pHLIP peptide family. Members of the peptide of pHLIP family follow a similar pattern in terms of peptide sequence: an N-terminal flanking region (yellow) consists mainly of polar residues; a transmembrane region (red) consists mainly of hydrophobic residues, but also includes protonatable residues which prevent insertion at physiological $\mathrm{pH}$; and a C-terminal flanking region (green), which may not present in all peptides, contains a few additional protonatable residues. Cargo can be conjugated either to the $\mathrm{C}$ - or $\mathrm{N}$ terminus via single cysteine or lysine residues. 
Box 2. Not only can pHLIP conjugates be constructed in a one-to-one ratio between pHLIP (blue) and cargo (red), but the pHLIP can also be used to coat molecules in order to increase biocompatibility, targeting, and to induce cellular uptake (Figure II). Nanoparticles, for instance, have a wide variety of uses in medical applications, including as drug-laden nanocarriers, as particles that enhance another form of therapy, and as therapeutic agents themselves. Decorating nanoparticles with multiple pHLIPs not only results in targeting specificity and greater uptake by cells in acidic, diseased environments, but can also bestow biocompatibility on what might be an otherwise non-biocompatible nanoparticle. pHLIPs could be used as decorations on a wide variety of nanoparticles, and have already been studied in lipid, polymer, and metal-based nanomaterials. 

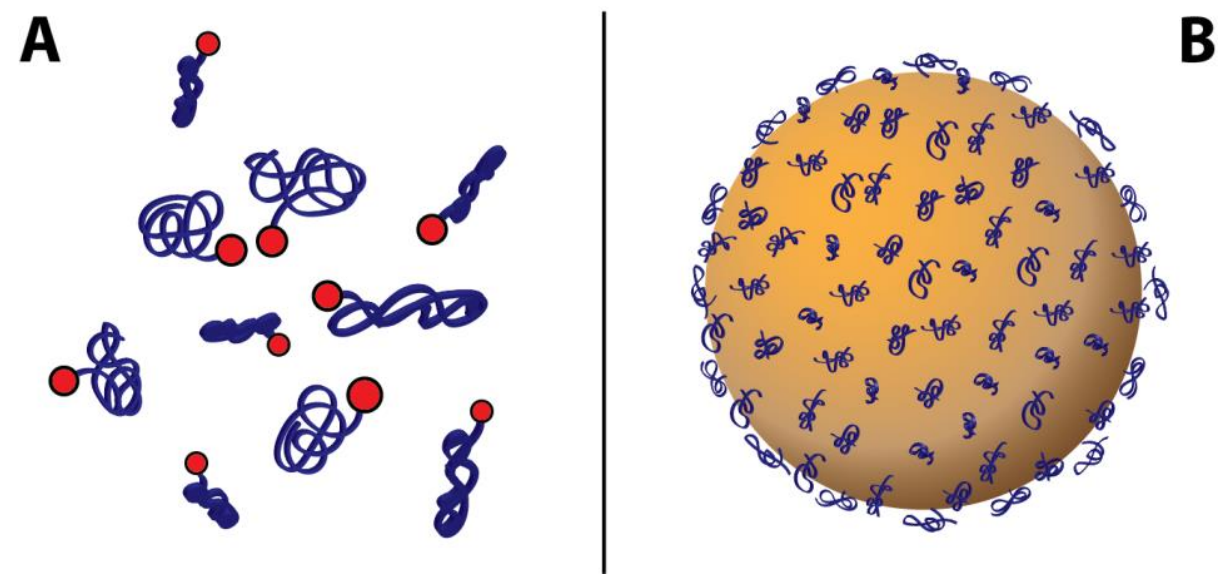

Figure II. pHLIP delivery capability. pHLIPs are used to increase the tumor targeting and delivery of various types of cargoes, such as imaging agents and therapeutics, by conjugating cargo to pHLIP in a one-to-one ratio (A). pHLIPs can also be used to decorate nanoparticles (B), to increase the biocompatibility, tumor targeting and promote cellular uptake of nanoparticles by cancer cells. 


\title{
CHAPTER 2
}

Published in Bioconjugate Chemistry on July 9, 2016

\section{PET Imaging of Extracellular pH in Tumors with ${ }^{64} \mathrm{Cu}$ - and ${ }^{18} \mathrm{~F}$ - labeled pHLIP ${ }^{\circledR}$ Peptides: A Structure-Activity Optimization Study}

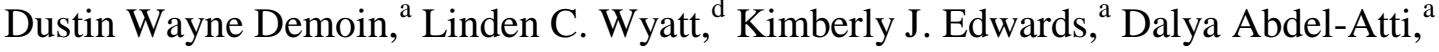 \\ Mirkka Sarparanta, ${ }^{\mathrm{a}}$ Jacob Pourat, ${ }^{\mathrm{a}}$ Valerie A. Longo, ${ }^{\mathrm{c}}$ Sean D. Carlin, ${ }^{\mathrm{a}, \mathrm{b}}$ \\ Donald M. Engelman, ${ }^{\mathrm{e}}$ Oleg A. Andreev, ${ }^{\mathrm{d}, \mathrm{e}}$ Yana K. Reshetnyak, ${ }^{\mathrm{d}, \mathrm{e}}$ \\ Nerissa Viola-Villegas, ${ }^{\mathrm{f}}$ and Jason S. Lewis ${ }^{*}$,a,b,g
}

${ }^{a}$ Department of Radiology, ${ }^{b}$ Program in Molecular Pharmacology, and ${ }^{c}$ SmallAnimal Imaging Core Facility, Memorial Sloan Kettering Cancer Center, 1275 York Avenue, New York, NY 10065, USA

${ }^{d}$ Physics Department, University of Rhode Island, 2 Lippitt Road, Kingston, RI 02881, USA

e pHLIP, Inc., 2 Lippitt Road, Kingston, RI 02881, USA

${ }^{\mathrm{f}}$ Department of Oncology, Karmanos Cancer Institute, Detroit, MI 48201, USA

${ }^{\mathrm{g}}$ Weill Cornell Medical College, 1300 York Avenue, New York, NY 10065, USA

* Corresponding Author: E-mail: lewisj2@mskcc.org. Tel: (646) 888-3038. Fax: (646) 888-3059. 
Author Contributions: The manuscript was written through contributions of all authors. All authors have given approval to the final version of the manuscript.

Notes: The authors declare the following competing financial interest(s): Drs. Andreev, Engelman, Lewis, and Reshetnyak have financial interests in pHLIP, Inc. Drs. Andreev and Reshetnyak have disclosed those interests fully to the University of Rhode Island. Dr. Engelman has disclosed those interests fully to Yale University. Dr. Lewis has disclosed those interests fully to the Memorial Sloan Kettering Cancer Center. Drs. Andreev, Engelman, Lewis, and Reshetnyak have in place an approved plan for managing any potential conflicts. 


\section{ABSTRACT}

$\mathrm{pH}$ (low) insertion peptides (pHLIP peptides) target acidic extracellular environments in vivo due to $\mathrm{pH}$-dependent cellular membrane insertion. Two variants (Var3 and Var7) and wild-type (WT) pHLIP peptides have shown promise for in vivo imaging of breast cancer. Two positron emitting radionuclides $\left({ }^{64} \mathrm{Cu}\right.$ and $\left.{ }^{18} \mathrm{~F}\right)$ were used to label the NOTA- and NO2A-derivatized Var3, Var7, and WT peptides for in vivo biodistribution studies in $4 \mathrm{~T} 1$ orthotopic tumor-bearing $\mathrm{BALB} / \mathrm{c}$ mice. All of the constructs were radiolabeled with ${ }^{64} \mathrm{Cu}$ or $\left[{ }^{18} \mathrm{~F}\right]-\mathrm{AlF}$ in good yield. The in vivo biodistribution of the 12 constructs in $4 \mathrm{~T} 1$ orthotopic allografted female BALB/c mice indicated that NO2A-cysVar3, radiolabeled with either ${ }^{18} \mathrm{~F}$ (4T1 uptake; $8.9 \pm$ $1.7 \% \mathrm{ID} / \mathrm{g}$ at $4 \mathrm{~h}$ p.i.) or ${ }^{64} \mathrm{Cu}(4 \mathrm{~T} 1$ uptake; $8.2 \pm 0.9 \% \mathrm{ID} / \mathrm{g}$ at $4 \mathrm{~h}$ p.i. and $19.2 \pm 1.8 \%$ ID/g at $24 \mathrm{~h}$ p.i.), shows the most promise for clinical translation. Additional studies to investigate other tumor models (melanoma, prostate, and brain tumor models) indicated the universality of tumor targeting of these tracers. From this study, future clinical translation will focus on ${ }^{18} \mathrm{~F}$ - or ${ }^{64} \mathrm{Cu}$-labeled NO2A-cysVar3. 


\section{INTRODUCTION}

Cancer cells, in general, prefer the glycolytic pathway of energy production as a consequence of their deregulated proliferative machinery and requirement for survival, which results in excess acidity. To maintain homeostasis, cancer cells release lactic acid, formed during glycolytic processes, to the extracellular environment.(1-3) The release of acid lowers the extracellular $\mathrm{pH}$ immediately surrounding these cells relative to the $\mathrm{pH}$ of normal tissues. Due to the differential in $\mathrm{pH}$ gradients, a probe marking these acidic regions in rapidly proliferating tissues can potentially distinguish cancerous from normal tissue.

The family of $\mathrm{pH}$ (low) insertion peptides (pHLIP peptides) represents a novel class of delivery agents which can target acidic malignant tissue.(4, 5) The molecular mechanism of targeting is based on the $\mathrm{pH}$-dependent formation of a transmembrane $\alpha$-helix, which is accompanied by the insertion of pHLIP peptides into the cellular membrane in environments with low extracellular $\mathrm{pH}$ (Figure 1).(6) Among investigated pHLIP variants, three were under consideration for clinical translation: wild-type (WT), variant 3 (Var3), and variant 7 (Var7).(7)

To date, pHLIP peptides have been conjugated to organic dyes or positron-emitting radioisotopes for in vitro and in vivo studies.(7-11) Viola-Villegas et al. showed that DOTA-pHLIP variants were able to target PC3-WT cancer cells in a pH-dependent manner using ${ }^{68} \mathrm{Ga}-\mathrm{DO} 3 \mathrm{~A}-\mathrm{cysWT}$ and ${ }^{68} \mathrm{Ga}-\mathrm{DO} 3 \mathrm{~A}-\mathrm{cys} V a r 7 .(12)$ Additionally, ${ }^{64} \mathrm{Cu}$ - 
NOTA-Var7 showed in vivo localization that was inversely related to measured extracellular $\mathrm{pH}$ (pHe), but with limited overall tumor uptake (1.36 $\pm 0.43 \% \mathrm{ID} / \mathrm{g}) .(12)$

The selection of NOTA as the chelator in the present studies opened opportunities to explore radiolabeling strategies with a variety of positron-emitting radiometals, as well as with ${ }^{18} \mathrm{~F}$, for positron emission tomography (PET) imaging.(13-16) As the previous studies with pHLIP-PET radiopharmaceuticals in preclinical models established the proof-of-principle that the pHLIP platform can indeed selectively target and image tumor acidosis, our goals have now advanced to performing first-in-human trials. It was first imperative, however, to optimize the tracer (increasing accumulation in the tumor with a concomitant clearance from nontarget tissues) by careful selection of the optimal radioisotope and pHLIP peptide variant combination prior to commencing clinical studies.

In this current study, we investigated and compared the pharmacokinetic properties of six pHLIP constructs (NOTA-WT, NOTA-Var3, NOTA-Var7, NO2A-cysWT, NO2Acys Var3, and NO2A-cysVar7) labeled with two different PET isotopes $\left({ }^{64} \mathrm{Cu}\right.$ and $\left.{ }^{18} \mathrm{~F}\right)$ using an orthotopic breast cancer model (murine 4T1 mammary adenocarcinoma, a model of human stage IV breast cancer) through ex vivo biodistribution and in vivo imaging studies. In addition, the biophysical measurements of the nonradioactive, metalated peptides were conducted to help explain the variations observed in the animal studies. From these studies, two lead radiopharmaceuticals $\left({ }^{18} \mathrm{~F}-\mathrm{AlF}-\mathrm{NO} 2 \mathrm{~A}-\right.$ 
cys Var3 and ${ }^{64} \mathrm{Cu}-\mathrm{NO} 2 \mathrm{~A}-\mathrm{cys} V$ ar3) were selected and further assessed in melanoma, prostate, and brain tumor models.

\section{RESULTS}

\section{Preparing the Radiotracers}

The NOTA (1,4,7-triazacyclononane- $\mathrm{N}^{\prime}, \mathrm{N}^{\prime \prime}, \mathrm{N}^{\prime \prime \prime}$-triacetic acid) chelator was conjugated to the pHLIP variants in two ways (Figure 2). The NOTA-pHLIP compounds contain NOTA with three carboxylic acid groups conjugated to the N-terminus of the peptide (Figure 2A). The NO2A-cyspHLIP compounds contain NOTA with two carboxylic acid groups, where the third group was used to conjugate the chelator to the peptide through the cysteine side chain (Figure 2B). The $\mathrm{Cu} 2+$ and AlF2+ NOTA complexes have an overall charge of -1 with a neutral charge at the N-terminus of the pHLIP peptides. All complexes with the NO2A-chelates have an overall neutral charge with the positive charge preserved at the N-terminus of the pHLIP peptides. The three peptide sequences are tabulated in Figure 2C.

The ${ }^{64} \mathrm{Cu}$ radiolabeling of each of the conjugates was achieved with $>60 \%$ (isolated)

yield with $>93 \%$ purity (Supporting Information); the ${ }^{18} \mathrm{~F}-\mathrm{AlF}$ radiolabeling of each of the conjugates was 4-50\% (isolated and decay corrected to start of synthesis) yield with $>95 \%$ purity (Supporting Information). Table S1 lists the specific activity of each radiolabeled pHLIP peptide.

\section{In Vivo Studies in 4T1 Tumor Models}


Direct evaluation of all 12 tracers in vivo was carried out in 4T1 orthotopic (surgically implanted) allografted female BALB/c mice in order to determine which compounds would be best translated into clinical studies. Figure 3 shows the in vivo PET imaging slices at the level of the tumor at $4 \mathrm{~h}$ p.i. of 11 of the 12 tracers tested and the ex vivo biodistribution in selected organs at 4 and 24 h p.i. (\%ID/g, mean \pm S.D.). The corresponding maximum intensity projections (MIPs) from the PET imaging study and the PET imaging slices at the level of the tumor with a lower maximum value for better visualization of the tumor at $4 \mathrm{~h}$ p.i. for the ${ }^{18} \mathrm{~F}-\mathrm{AlF}-\mathrm{NO} 2 \mathrm{~A}$-cyspHLIP constructs are collected in Figure S1. The $4 \mathrm{~h}$ ex vivo biodistribution values (\% ID/g, mean \pm S.D.) are collected in Tables $\mathbf{1}$ and $\mathbf{2}$ for all 12 radiopharmaceuticals, and the complete biodistribution results for all time points are tabulated in the Supporting Information (Tables S1-1 through S13-14). Due to the ex vivo biodistribution data for ${ }^{64} \mathrm{Cu}-\mathrm{NOTA}-\mathrm{Var} 7$ and the previous results from the imaging study of the other NOTA-pHLIP constructs, an additional imaging study for ${ }^{64} \mathrm{Cu}-\mathrm{NOTA}-\mathrm{Var} 7$ was unjustified. These data clearly indicate that all constructs with the NO2A chelator far outperform the constructs with the NOTA chelator. It appears that NO2A-cyspHLIP constructs have a longer blood half-life, which results in higher targeting and longer retention within the tumor while the radiopharmaceutical clears from nontarget tissues and the blood.

\section{Biophysical Studies}

With 1-palmitoyl-2-oleoyl-sn-glycero-3-phosphocholine (POPC) liposomes as model membranes, our comparative biophysical investigation of the $\mathrm{pH}$-dependent 
interaction of NOTA-pHLIP and NO2A-cyspHLIP constructs with the lipid bilayer indicated that NOTA constructs adopt unfavorable conformations at the membrane surface at physiological and low $\mathrm{pH}$ compared to NO2A constructs. Although biophysical measurements are typically conducted in solutions without the further addition of ions, we chose to investigate the performance of these constructs in both the absence and the presence of physiological levels of free magnesium and calcium ions. Wild-type constructs appear to be sensitive to the presence of divalent cations, most likely due to the multiple protonatable aspartic and glutamic acid residues at the inserting end of the peptide, which tend to form divalent complexes with these ions at physiological $\mathrm{pH}$. Plots demonstrating the changes in fluorescence spectra of AlFNOTA-Var3, Cu-NOTA-Var3, AlF-NO2A-cysVar3, and Cu-NO2A-cysVar3 upon interaction with membranes are shown in Figure S2.

Figure 3 shows the biodistribution of ${ }^{64} \mathrm{Cu}$ - and ${ }^{18} \mathrm{~F}-\mathrm{NO} 2 \mathrm{~A}$ constructs in selected tissues at 4 and $24 \mathrm{~h}$. As previously reported,(17) Var7 constructs exhibit the fastest blood clearance with the highest signal in the kidneys at $4 \mathrm{~h}$ p.i. and kidney clearance at later time points. Both ${ }^{18} \mathrm{~F}-\mathrm{AlF}-\mathrm{NO} 2 \mathrm{~A}-\mathrm{cys} \mathrm{WT}$ and ${ }^{64} \mathrm{Cu}-\mathrm{NO} 2 \mathrm{~A}-\mathrm{cysWT}$ have slightly higher liver and spleen uptake compared to the corresponding cysVar3 and cysVar7 conjugates. All of the NO2A constructs demonstrate a pH-dependent interaction with the lipid bilayer of the cellular membrane (Figures S3 and S4), but the NO2A-cysWT constructs exhibit the highest affinity to the lipid membrane at physiological $\mathrm{pH}$. Additionally, the $\mathrm{pK}$ of transition from the membrane-bound state to the inserted state is higher for NO2A-cysWT constructs compared with NO2A- 
cysVar3 and NO2A-cysVar7 constructs. This shift in $\mathrm{pK}$ is most likely responsible for the higher uptake of the NO2A-cysWT constructs by the liver and spleen.

Both ${ }^{64} \mathrm{Cu}-\mathrm{NO} 2 \mathrm{~A}$-cysVar3 and ${ }^{18} \mathrm{~F}-\mathrm{AlF}-\mathrm{NO} 2 \mathrm{~A}$-cysVar3 had the highest tumoral uptake, prolonged retention in the tumor, and minimal accumulation in other organs based on the ex vivo biodistribution data. Figure 4 shows the pH-dependent insertion and tabulates the percentage of the NO2A-cysVar3 construct population that is inserted in the membrane at various pHs. The results indicate that less than 5\% of the lead compounds should be inserted into the membrane at $\mathrm{pH} 7.4$ (physiological $\mathrm{pH}$ ), whereas greater than $25 \%$ of the NO2A-cysVar3 compounds should be inserted into the cellular membrane at $\mathrm{pH} 6.0$ (the approximate $\mathrm{pH}$ at the surface of cancer cells). In addition, the membrane insertion of Var3 and Var7 takes place at a rate 2 orders of magnitude higher than the rate of insertion of WT.(17)

\section{In Vivo Studies in Prostate, Melanoma, and Brain Tumor Models}

The two NO2A-cys Var3 compounds that showed superior targeting of the 4T1 tumors were compared in melanoma and prostate cancer models. Figure 5 shows the in vivo PET images of the uptake of the two tracers in tumors at 4 and 24 h p.i. The graph in Figure 6 directly compares the tumoral uptake of the two lead compounds at 4, 6, and $24 \mathrm{~h}$ p.i. for four of the tumor models investigated in this study. Due to the large variation in tumor size for the $\mathrm{LNCaP}$ tumors, the ex vivo data shows a minimal increase in tumoral uptake over the PC3 tumors, but both the murine B16-F10 
melanoma tumors and 4T1 breast cancer tumors showed greater uptake of the tracers compared to the human prostate cancer models.

Because of the low uptake of the tracers in brain tissue, the ${ }^{18} \mathrm{~F}-\mathrm{AlF}-\mathrm{NO} 2 \mathrm{~A}-\mathrm{cys}$ Var3 was also evaluated in U87MG tumor-bearing mice. These tumors were implanted into the brain cavity or xenografted subcutaneously on the flank of nude mice. Figure S5 shows the imaging of these tumors. The uptake in the U87MG tumors that were implanted into the brain cavity showed significant uptake of the tracer in the portion of the tumor that was outside the skull. During necropsy, these tumors did not show significant infiltration of the brain. The ex vivo biodistribution of ${ }^{18} \mathrm{~F}-\mathrm{AlF}-\mathrm{NO} 2 \mathrm{~A}-$ cys Var3 at $6 \mathrm{~h}$ p.i. in the nude mice with flank tumors is included in Figure S5. In order to conclusively determine if the uptake of ${ }^{18} \mathrm{~F}$-AlF-NO2A-cys Var3 was only in the portion of the tumor outside of the skull, a group of mice with GSC 5-22 tumors orthotopically xenografted behind an intact blood-brain barrier (BBB) were obtained. During necropsy, these tumors had infiltrated half of the brain, but there was no uptake observed in the PET image (Figure S5).

\section{Specific Activity Investigation}

The tracers were prepared to have comparable specific activities (approximately 100 $\mu \mathrm{Ci} / \mathrm{nmol}(3.7 \mathrm{MBq} / \mathrm{nmol}))$ for most of the studies. A few additional studies were conducted with higher and lower specific activities in order to compare the effect of specific activity on tumoral uptake. The data sets were analyzed individually and showed that specific activity did not impact the biodistribution of the tracers, 
especially tumoral uptake. Thus, all of the data points for similar tumor models were averaged in aggregate (regardless of specific activity) and tabulated together in the Supporting Information.

\section{Tissue Autoradiography}

The uptake of ${ }^{64} \mathrm{Cu}-\mathrm{NO} 2 \mathrm{~A}-\mathrm{cys} V a r 3$ (24 h p.i.) in LNCaP and PC3 tumors was investigated via autoradiography with viable tissue stained with hematoxylin and eosin (H\&E) to determine if the uptake was specific to regions showing indications of metabolic stress or necrosis. Interestingly, uptake of ${ }^{64} \mathrm{Cu}-\mathrm{NO} 2 \mathrm{~A}-\mathrm{cys} V$ ar3 appears to correlate with necrotic regions in $\mathrm{LNCaP}$ tumors, but not in PC3 tumors (Figure 7). This could be a result of the PC3 tumors having less necrosis overall than the LNCaP tumors in this study. Additionally, the autoradiography shows increased relative uptake of ${ }^{64} \mathrm{Cu}-\mathrm{NO} 2 \mathrm{~A}-\mathrm{cys} \operatorname{Var} 3$ (24 h p.i.) in LNCaP over PC3 tumors (Figure S6). This finding is similar to the results from previous studies by our group, in which both LNCaP and PC3 tumors were investigated.(10-12) H\&E staining of tumor sections from 4T1 tumors excised 5-12 days post-inoculation show non-necrotic tumor tissue; thus, the greater tumoral uptake of the ${ }^{64} \mathrm{Cu}-\mathrm{NO} 2 \mathrm{~A}-\mathrm{cys} V a r 3$ (according to the ex vivo biodistribution data) does not appear to be correlated to necrosis.

\section{DISCUSSION}

Two positron-emitting radionuclides $\left({ }^{18} \mathrm{~F}\right.$ and $\left.{ }^{64} \mathrm{Cu}\right)$ can be used to label NOTApeptides for in vivo imaging. While ${ }^{64} \mathrm{Cu}\left(\mathrm{t}_{1 / 2}=12.7 \mathrm{~h}\right)$ has a moderately short half-life, the production of ${ }^{64} \mathrm{Cu}$ requires a higher energy cyclotron and expensive targetry with 
automated radionuclidic separation which many institutions do not currently have. Conversely, ${ }^{18} \mathrm{~F}\left(\mathrm{t}_{1 / 2}=109.8 \mathrm{~min}\right)$ is readily available from most medical cyclotrons and radiopharmaceutical centers, is routinely produced at numerous sites worldwide, and can be inexpensively concentrated for radiolabeling. Unlike conventional nucleophilic ${ }^{18} \mathrm{~F}$ labeling of small organic molecules, using a chelator to coordinate the aluminum center of $\left[{ }^{18} \mathrm{~F}\right]$-AlF is straightforward and can be done in aqueous solution. $(15,16)$

After the radiopharmaceuticals were successfully synthesized, their ability to target cancerous tissue was directly compared in $4 \mathrm{~T} 1$ tumor-bearing female $\mathrm{BALB} / \mathrm{c}$ mice. This model of triple-negative human stage IV breast cancer was chosen based on previous findings in our group that the pHLIP-PET agents were excreted through both the hepatobiliary and renal pathways and showed very good tumor:muscle ratios.(1012) As such, breast cancer seemed an appropriate target which is imaged in humans away from these excretion organs. Additionally, previous results from Serganova et al. indicated that the $\mathrm{pH}$ of $4 \mathrm{~T} 1$ tumors increases with increased size.(18) Thus, the $4 \mathrm{~T} 1$ tumors in this study were purposely used 5-7 days after inoculation to ensure lower tumoral $\mathrm{pH}$. The ex vivo biodistribution data show that the metalated NOTA-pHLIP variants are quickly cleared via both the hepatobiliary and renal pathways to reduce the overall circulating radioactivity significantly by $12 \mathrm{~h}$ p.i. The rapid excretion of the ${ }^{64} \mathrm{Cu}$-NOTA-pHLIP variants from the intestines and slow elimination from the kidneys over time indicate that the compounds are not being taken up by the cells within these organs, but are being eliminated via these pathways. Additionally, when 
comparing the $2 \mathrm{~h}$ ex vivo data for kidneys-to-large intestine ratios $\left[{ }^{64} \mathrm{Cu}-\mathrm{NOTA}\right.$-WT is $0.35 \pm 0.11 ;{ }^{18} \mathrm{~F}$-AlF-NOTA-WT is $0.42 \pm 0.03 ;{ }^{64} \mathrm{Cu}$-NOTA-var3 is $0.29 \pm$ $0.12 ;{ }^{18} \mathrm{~F}$-AlF-NOTA-var3 is $0.52 \pm 0.15 ;{ }^{64} \mathrm{Cu}$-NOTA-var7 is $0.32 \pm 0.07 ;{ }^{18} \mathrm{~F}$-AlFNOTA-var7 is $0.55 \pm 0.19$ ], there is no significant difference between the excretion pathways of variants radiolabeled with ${ }^{64} \mathrm{Cu}$ or ${ }^{18} \mathrm{~F}$. Perhaps the charge of the complexes appended to the pHLIP peptides helps to improve the elimination of these compounds, but also decreases the likelihood of uptake in low $\mathrm{pH}$ environments, such as the tumor, which may require more residence time in the blood. The lack of tumor targeting is evident in the imaging study results shown in Figure 3.

Conversely, the neutrally charged NO2A complexes conjugated to the cysteine residue of pHLIP peptides (which preserve their positive charge at the N-terminus) show prolonged blood circulation with slower clearance, consequently leading to better perfusion of the tracers in the tumor microenvironment where they are effectively trapped. The steady increase of the tumoral uptake of each of the tracers implies slow localization in the tumor site. The radiometalated NO2A-cysVar7 compounds had the shortest blood retention with similar tumoral uptake at $4 \mathrm{~h}$ to the other radiolabeled compounds. This rapid blood clearance may make the radiometalated NO2A-cysVar7 compounds useful for shorter imaging times (tumor is visible in the slices at the tumor level in Figure S1) as long as the tumor is sufficiently distant from the kidneys (the major organ visible in the MIP in Figure S1). The rapid clearance of the Var7 constructs (noted in previous studies $(17,19)$ ) may be a function of their physical characteristics. While these compounds have pKs of insertion similar to the NO2A- 
cys Var3 constructs, they are less hydrophobic than the NO2A-cysVar3 constructs. This lower hydrophobicity results in faster blood clearance (indicated by lower amounts of NO2A-cysVar7 constructs in the blood at every time point). The lower amount of radiolabeled compound in the blood for longer periods of time may result in lower tumoral uptake of the NO2A-cysVar7 constructs because the targeted tissue is exposed to less of the construct. At the same time, NO2A-cysWT compounds exhibited highest accumulation in liver and spleen compared to NO2A-cysVar3 and NO2A-cysVar7, most probably due to their higher membrane affinity (greater hydrophobicity) at physiological $\mathrm{pH}$ and slower rate of membrane insertion.(17) Additionally, the ${ }^{64} \mathrm{Cu}-\mathrm{NO} 2 \mathrm{~A}-\mathrm{cyspHLIP}$ derivatives show decreased radioactivity accumulation in the kidney from $4 \mathrm{~h}$ p.i. to $24 \mathrm{~h}$ p.i. which is due to clearance of the drug rather than uptake, whereas the liver and spleen uptake remains similar for the two time points. The liver and spleen uptake may be due to loss of the ${ }^{64} \mathrm{Cu}$ from the chelator,(20) digestion of the radiotracer in vivo, or accumulation of intact tracer in these organs. Further investigation of this uptake is currently underway by investigating a pHLIPcys-NO2A derivative that contains the cysteine residue on the internalized portion of the peptide (C terminus), which could result in lower liver and spleen uptake if the uptake in these organs is due to loss of the ${ }^{64} \mathrm{Cu}$ from the chelator after membrane insertion.

From these results it becomes evident that introducing a benzene ring in close proximity to the $\mathrm{N}$-terminus of the peptide sequence and using a negatively charged metal complex should be avoided in pHLIP peptide-mediated tumor imaging 
pharmaceuticals. Instead, conjugating to either the cysteine side chain or utilizing a suitable linking group on the $\mathrm{N}$-terminus may improve tumor targeting of the synthesized pHLIP peptide. These general guidelines may help explain the improvements seen in this study and understand the results from previous studies.

Two compounds from the 12 tracers included in this study showed the greatest tumoral uptake and significant tumor-to-background contrast in the images throughout the imaging studies: ${ }^{64} \mathrm{Cu}-\mathrm{NO} 2 \mathrm{~A}-\mathrm{cys} V a r 3$ and ${ }^{18} \mathrm{~F}-\mathrm{AlF}-\mathrm{NO} 2 \mathrm{~A}-\mathrm{cys}$ Var3. Therefore, we next investigated the in vivo properties of these two tracers in PC3 and LNCaP tumorbearing mice to compare tumoral uptake with previous studies.(10-12) In these tumor models, the tumor accumulation of ${ }^{64} \mathrm{Cu}-\mathrm{NO} 2 \mathrm{~A}$-cysVar3 and ${ }^{18} \mathrm{~F}$-AlF-NO2A-cysVar3 was significantly higher compared to previous generations of PET isotope-labeled pHLIP constructs, especially at $24 \mathrm{~h}$ p.i. (Figure S7). Of particular note is the uptake of the ${ }^{18}$ F-py-click-6Ahx-WT reported by Daumar et al.(11) and ${ }^{68}$ Ga-DO3A-cysVar7 reported by Viola-Villegas et al.(12) Both of these previously reported compounds show significant uptake at $4 \mathrm{~h}$ p.i. and could be clinically useful. By comparison of the tumor:tissue ratios with those of previously reported compounds (Figure S8), the tumor:muscle ratios at $4 \mathrm{~h}$ p.i. were $7.4 \pm 1.3\left({ }^{64} \mathrm{Cu}-\mathrm{NO} 2 \mathrm{~A}\right.$-cysVar3 $), 6.9 \pm 1.9\left({ }^{18} \mathrm{~F}\right.$ AlF-NO2A-cysVar3), $4 \pm 2\left({ }^{18}\right.$ F-py-click-6Ahx-WT), and $1.1 \pm 1.5\left({ }^{68} \mathrm{Ga}-\mathrm{DO} 3 \mathrm{~A}-\right.$ cysVar7) in PC3 tumor-bearing male nude mice; the tumor:bone ratios were $2.7 \pm 0.6$ $\left({ }^{64} \mathrm{Cu}-\mathrm{NO} 2 \mathrm{~A}\right.$-cysVar3), $3.2 \pm 0.7\left({ }^{18} \mathrm{~F}\right.$-AlF-NO2A-cysVar3), $1.3 \pm 0.4\left({ }^{18} \mathrm{~F}\right.$-py-click6Ahx-WT), and $0.4 \pm 0.3\left({ }^{68} \mathrm{Ga}-\mathrm{DO} 3 \mathrm{~A}-\mathrm{cys} V\right.$ ar7 $)$ in PC3 tumor-bearing male nude mice. Additionally, the tumor:muscle ratios in $\mathrm{LNCaP}$ tumor-bearing male nude mice 
at $4 \mathrm{~h}$ p.i. were $6 \pm 2\left({ }^{64} \mathrm{Cu}-\mathrm{NO} 2 \mathrm{~A}\right.$-cys Var3 $), 4.7 \pm 0.9\left({ }^{18} \mathrm{~F}-\mathrm{AlF}-\mathrm{NO} 2 \mathrm{~A}\right.$-cys Var3 $)$, and $6 \pm 3\left({ }^{18}\right.$ F-py-click-6Ahx-WT); the tumor:bone ratios in LNCaP tumor-bearing male nude mice at $4 \mathrm{~h}$ p.i. were $4.2 \pm 1.7\left({ }^{64} \mathrm{Cu}-\mathrm{NO} 2 \mathrm{~A}\right.$-cysVar 3$), 4.3 \pm 1.1\left({ }^{18} \mathrm{~F}-\mathrm{AlF}-\mathrm{NO} 2 \mathrm{~A}-\right.$ cysVar3), and $1.8 \pm 0.6\left({ }^{18}\right.$ F-py-click-6Ahx-WT). In each of these cases, the tumor ratios of the two lead compounds from this study are much better than the ${ }^{68} \mathrm{Ga}-$ DO3A-cysVar7, which would result in improved image contrast. While the ${ }^{18} \mathrm{~F}$-pyclick-6Ahx-WT had similar tumor:muscle ratios, the tumor:bone ratios were much lower than the two lead compounds in both prostate cancer tumor models. The decreased tumor:bone ratios may indicate that the previously reported ${ }^{18} \mathrm{~F}$-py-click6Ahx-WT was more prone to defluorination than the ${ }^{18} \mathrm{~F}$-labeled tracers presented in this study.

Despite the similar tumor-targeting properties, the preparation of ${ }^{18} \mathrm{~F}$-py-click-6AhxWT requires two HPLC-based separations and generates a volatile radioactive intermediate. The preparation of either of the lead compounds from this work, in contrast, did not required HPLC purification or heating beyond $80{ }^{\circ} \mathrm{C}$ which is optimal for rapid dose-on-demand production of the tracers for patient trials. Thus, the two lead compounds from this study appear to be the best candidates for clinical translation because of their improved biopharmaceutical properties and facile radiosynthesis.

Two other tumor types (melanoma and glioma) were included in this study to determine if the high uptake of these radiotracers seen in breast and prostate cancer 
models would translate to other types of tumors. The lead compounds have excellent tumor:background contrast in B16-F10 murine melanoma tumor-bearing mice, which was useful to identify very small tumors $(<17 \mathrm{mg} ; \sim 4 \mathrm{~mm} 3)$ in the PET image. Because of the contrast and high uptake, we plan to continue investigating the use of the two lead compounds in metastatic B16-F10 tumor models. For the brain tumor models, both an orthotopic and a subcutaneous (flank) brain tumor mouse model indicated that the ${ }^{18} \mathrm{~F}-\mathrm{AlF}-\mathrm{NO} 2 \mathrm{~A}-\mathrm{cys}$ Var3 was unable to penetrate an intact $\mathrm{BBB}$, but was able to target brain tumors that had developed outside the BBB (Figure S5). Because this is a peptide-based tracer, we did not expect the compound to be able to cross an intact $\mathrm{BBB}$; thus, these tracers may be useful in identifying metastases and possibly brain tumors in situations of compromised BBBs.

Previously, autoradiography was performed by Viola-Villegas et al. in prostate tumor sections from excised PC3 and LNCaP tumors in order to show differences in histology and tracer uptake;(12) thus, a similar study was performed with the uptake of ${ }^{64} \mathrm{Cu}-\mathrm{NO} 2 \mathrm{~A}$-cysVar3 in the same tumor lines. Overall, the ex vivo tumor sections show increased uptake of the ${ }^{64} \mathrm{Cu}-\mathrm{NO} 2 \mathrm{~A}$-cysVar3 in the necrotic regions of the LNCaP tumors (Figure 7). This may indicate that if necrosis is present, then uptake of ${ }^{64} \mathrm{Cu}-\mathrm{NO} 2 \mathrm{~A}$-cysVar3 may increase, but is not the only requirement. The findings here generally match the trend found by Viola-Villegas et al. in prostate tumor sections.(12) Additionally, the greater uptake in the allografted tumors may indicate the relative overall tumor environment is less acidic (higher) in the slower growing 
human cell lines investigated (PC3 and LNCaP), but is more acidic (lower) in the faster growing murine cell lines (B16-F10 and 4T1).

\section{EXPERIMENTAL SECTION}

The D-amino acid versions of NOTA-derivatized pHLIP peptides were purchased from CS Bio (Menlo Park, CA) with $\geq 95 \%$ purity (combination of both chelators and three variants from Figure 2: NOTA-WT, NOTA-Var3, NOTA-Var7, NO2A-cysWT, NO2A-cysVar3, and NO2A-cysVar7). All other chemicals were purchased from commercial suppliers without further purification unless otherwise stated. Detailed method descriptions are found in the Supporting Information.

\section{Radiochemistry}

\section{General Labeling Methods for ${ }^{64} \mathrm{Cu}$-NOTA-pHLIP}

Following a preparation similar to that of Zeglis et al.,(21) ${ }^{64} \mathrm{Cu}$ was diluted in a 100 $\mathrm{mM}$ NH4OAc buffer $(\mathrm{pH} \sim 5.5)$ and reacted with a NOTA-pHLIP derivative at $80{ }^{\circ} \mathrm{C}$ for $15 \mathrm{~min}$.

\section{General Labeling Methods for ${ }^{18}$ F-AlF-NOTA-pHLIP}

Following a modified labeling method described by Dijkgraaf et al.,(22) ${ }^{18} \mathrm{~F}$ from the cyclotron target water was concentrated using a chromafix cartridge into $0.1 \mathrm{~mL}$ of 0.4 M KHCO3 in metal-free water. The $\mathrm{pH}$ was adjusted and $0.1 \mathrm{~mL} \mathrm{AcN}$ added. To this solution, $\mathrm{AlCl} 3(80 \mathrm{nmol})$ in $0.1 \mathrm{M} \mathrm{NH} 4 \mathrm{OAc}$ buffer $(\mathrm{pH} \sim 4.1)$ was added. After 5 
min, the NOTA-pHLIP derivative was added and the reaction mixture reacted at $75^{\circ} \mathrm{C}$ for $15 \mathrm{~min}$.

\section{General Purification}

An Oasis HLB Plus Light or C18 Sep-Pak Light cartridge (Waters, Milford, MA) was used to remove unbound activity. The pure radiolabeled NOTA-pHLIP derivatives were eluted with EtOH and diluted with sterile phosphate buffered saline (PBS) with radiochemical purities $>93 \%$ before administration to animals.

\section{Animal Models}

Animal studies were conducted according to an MSKCC IACUC-approved animal protocol. 4T1 (orthotopic breast cancer allografts of $1 \times 106$ cells surgically implanted in the mammary fat pad) and B16-F10 (orthotopic melanoma allografts of $1 \times$ 105 cells injected intradermally on the shoulder) tumors were orthotopically inoculated in media in female BALB/c and C57B1/6 mice (Charles River Laboratories $(\mathrm{CRL}))$, respectively. Additionally, PC3 $(3 \times 106$ cells $)$ or LNCaP $((3-6) \times 106$ cells $)$ tumors were subcutaneously xenografted on the shoulder of athymic nude male mice (CRL). Mice were used once tumor volumes were approximately 90-300 mm3.

\section{In Vivo Animal PET Imaging and Biodistribution}

Mice were injected with radiolabeled NOTA-pHLIP derivatives i.v. (500-700 $\mu \mathrm{Ci} /$ mouse for PET imaging studies and $25-100 \mu \mathrm{Ci} /$ mouse for biodistribution studies). All injections were less than $200 \mu \mathrm{L}$ with $<10 \%$ EtOH in sterile PBS. PET 
images were obtained with the mice under anesthesia in an Inveon PET-CT or microPET Focus 120 (Siemens) at $0.5-48$ h p.i. All images were analyzed using ASIPro VM (Concorde Microsystems). Dissections for ex vivo biodistribution were performed on mice after $\mathrm{CO} 2$ asphyxiation or cervical dislocation while anesthetized at reported time points. Weight of the syringe prior to injection and after injection was used to determine the mass of injectate. Activity of the syringe prior to injection and after injection was used to determine the percent of injectate administered. The mass injected was corrected by the percent of radioactivity injected. Four to five aliquots $(10 \mu \mathrm{L})$ were weighed and counted as internal standards for each study. All of the collected organs were counted using an automatic gamma counter (Wizard 3", PerkinElmer, Waltham, MA). The total injected dose was found as the mass injected dose $\times$ fraction radioactivity injected $\times$ internal standard average counts/g. The percent injected dose $(\% \mathrm{ID})$ was determined as the counts for the tissue $\times 100 /$ total injected dose. The $\% \mathrm{ID} / \mathrm{g}$ was calculated as the $\% \mathrm{ID} /$ tissue weight. The average and standard deviation of the \% ID and $\% \mathrm{ID} / \mathrm{g}$ was determined using normal methods $(\mathrm{n}-1)$ for each set of mice.

\section{Ex Vivo Autoradiography, Staining, and Microscopy}

Tumors were excised from the mouse, embedded into Tissue-Plus O.C.T. (Scigen, Gardena, CA), stored at $-20{ }^{\circ} \mathrm{C}$ until sectioning, and cut in sequential $10 \mu \mathrm{m}$ sections. Select sections were exposed to autoradiography film (Fujifilm, GE Healthcare) for 13 days and read using a typhoon photographic film scanner (GE Healthcare). 
Additionally, sections were stained with $\mathrm{H} \& \mathrm{E}$ for gross tumor microscopy and then scanned.

\section{Biophysical Studies}

Biophysical studies were conducted on nonradioactive standards of the NOTA-pHLIP and NO2A-cyspHLIP compounds. All experiments were conducted with a $5 \mu \mathrm{M}$ to 1 $\mathrm{mM}$ construct to lipid ratio. State I (construct with no liposomes in solution) and State II (construct in the presence of liposomes) were measured at $\mathrm{pH} 8$; for State III (construct in the presence of liposomes), the $\mathrm{pH}$ of solution was dropped to $\mathrm{pH} 4 \mathrm{using}$ $2 \mathrm{M} \mathrm{HCl}$. Experiments were conducted in $10 \mathrm{mM}$ phosphate buffer (Sigma-Aldrich) either without additional ions or in the presence of physiological levels of free magnesium and calcium ( $0.65 \mathrm{mM}$ and $1.25 \mathrm{mM}$, respectively).

\section{Liposome Preparation}

Large unilamellar vesicles were prepared by extrusion. 1-Palmitoyl-2-oleoyl-snglycero-3-phosphocholine (POPC; Avanti Polar Lipids) was dissolved in chloroform at a concentration of $12.5 \mathrm{mg} / \mathrm{mL}$, desolvated by rotary evaporation, and dried under high vacuum for $2 \mathrm{~h}$. The phospholipid film was then rehydrated in $10 \mathrm{mM}$ phosphate buffer ( $\mathrm{pH} 8.0$ with or without ions), vortexed, and extruded $15 \times$ through a $50 \mathrm{~nm}$ pore.

\section{Steady-State Fluorescence}


Tryptophan fluorescence spectra were measured using a PC1 ISS spectrofluorometer (Champaign, IL) with temperature control set to $25^{\circ} \mathrm{C}$. Fluorescence spectra were recorded with excitation and emission slits set to $1 \mathrm{~mm}$, using an excitation wavelength of $295 \mathrm{~nm}$, with excitation and emission polarizers set to $54.7^{\circ}$ and $0^{\circ}$, respectively.

\section{Steady-State Circular Dichroism}

Circular dichroism measurements were taken using a MOS-450 spectrometer (BioLogic SAS, Claix, France) with temperature control set to $25^{\circ} \mathrm{C}$ in the range of 190 to $260 \mathrm{~nm}$ with step of $1.0 \mathrm{~nm}$.

\section{pH Dependence}

The pH-dependent insertion of the construct population was investigated using the shift in position of the wavelength of maximum intensity of the tryptophan fluorescence spectra varying $\mathrm{pH}$ conditions between 4 and 8 . The $\mathrm{pH}$ after addition of $\mathrm{HCl}$ was measured using an Orion PerpHecT ROSS Combination pH Micro Electrode and an Orion Dual Star pH and ISE Benchtop Meter (Thermo Fisher Scientific). Tryptophan fluorescence spectra were recorded at each $\mathrm{pH}$ value and were analyzed using an online protein fluorescence and structural toolkit (PFAST) to obtain the positions of spectral maxima ( $\lambda \max )$. Finally, the positions of $\lambda \max$ were plotted as a function of the various $\mathrm{pHs}$ and the Henderson-Hasselbalch equation employed to fit the data $\lambda_{\max }=\lambda_{\max }^{2}+\frac{\lambda_{\max }^{1}-\lambda_{\text {max }}^{2}}{1+10^{\left(\hat{p}^{2}+\mathrm{F}^{2}\right)}}$, where $\lambda \max 1$ and $\lambda \max 2$ are the positions of spectral 
maxima at the end and beginning of the State II - State III transition, respectively, $\mathrm{n}$ is the cooperativity parameter, and $\mathrm{pK}$ is the midpoint of transition.

\section{CONCLUSIONS}

In our study, the NO2A-cys Var3 is the lead construct for clinical investigation. This construct can be radiolabeled with ${ }^{18} \mathrm{~F}$ or ${ }^{64} \mathrm{Cu}$ with similar pharmacokinetic properties. The availability, limited dose exposure due to its short half-life, and cost effectiveness of ${ }^{18} \mathrm{~F}$ supports the utility of ${ }^{18} \mathrm{~F}$-AlF-NO2A-cysVar3 in the clinic. Nevertheless, ${ }^{64} \mathrm{Cu}-\mathrm{NO} 2 \mathrm{~A}$-cysVar3 may be more suitable for inpatient imaging (where the patient will be in the clinic longer than $24 \mathrm{~h}$ ) and in centers where the availability of the PET scanner may impact the time when patients are imaged. Clinical evaluation of these radiopharmaceuticals is currently underway.

\section{ACKNOWLEDGEMENT}

We appreciate the funding from NIH F32 CA186721 (D.W.D.), NIH R01 CA138468 (J.S.L.), the Academy of Finland (decision no. 278056, M.S.), NIH MSKCC Center Grant (P30-CA08748), and NIH Shared Instrumentation Grants (S10 RR020892-01 and S10 OD016207-01) for making this study possible. The authors would like to thank Prof. Fred K. Miller (Karmanos Cancer Institute, Detroit, MI) for his permission to obtain the 4T1 cancer cells and the Blasberg Lab (MSKCC, New York, NY) for providing us a frozen vial,(18) Alex Boländer (MSKCC, New York, NY) for providing U87MG tumor-bearing mice with tumors that were partially contained within the skull, and the Mellinghoff lab (MSKCC, New York, NY) for orthotopic GSC 5-22 tumors that were orthotopically implanted behind an intact BBB. 


$\begin{array}{ll}\text { ABBREVIATIONS } & \\ \text { AcN } & \text { acetonitrile } \\ \text { H\&E } & \text { hematoxylin and eosin } \\ \text { MIP } & \text { maximum intensity projection } \\ \text { NOTA } & 1,4,7-\text { triazacyclononane- } \mathrm{N}^{\prime}, \mathrm{N}^{\prime \prime}, \mathrm{N}^{\prime \prime \prime} \text {-triacetic acid } \\ \text { pHLIP } & \text { pH (low) insertion peptide } \\ \text { pHLIP-PET } & \text { pHLIP radiolabeled with a positron emitting nuclide }\end{array}$




\section{REFERENCES}

1. Cairns, R., Papandreou, I., and Denko, N. (2006) Overcoming physiologic barriers to cancer treatment by molecularly targeting the tumor microenvironment Mol. Cancer Res. 4, 61-70, DOI: 10.1158/1541-7786.MCR-06-0002

2. Gatenby, R. A. and Gillies, R. J. (2004) Why do cancers have high aerobic glycolysis? Nat. Rev. Cancer 4,891-899, DOI: 10.1038/nrc1478

3. Griffiths, J. R., McIntyre, D. J. O., Howe, F. A., and Stubbs, M. (2001) Why are cancer acidic? A carrier-mediated diffusion for $H+$ transport in the interstitial fluid, In the Tumor Microenvironment: Causes and Consequences of Hypoxia and Acidity (Gillies, R. $\quad$ J., $\quad$ Ed.) $\quad$ pp 46-62, $\quad$ Vol. 240, Novartis Foundation Symposia, John Wiley and Sons, Chichester.

4. Andreev, O. A., Engelman, D. M., and Reshetnyak, Y. K. (2010) $p H$-sensitive membrane peptides (pHLIPs) as a novel class of delivery agents Mol. Membr. Biol. 27, 341-352, DOI: 10.3109/09687688.2010.509285

5. Andreev, O. A., Engelman, D. M., and Reshetnyak, Y. K. (2014) Targeting diseased tissues by pHLIP insertion at low cell surface $\mathrm{pH}$. Front. Physiol. 5, DOI: 10.3389/fphys.2014.00097

6. Andreev, O. A., Dupuy, A. D., Segala, M., Sandugu, S., Serra, D. A., Chichester, C. O., Engelman, D. M., and Reshetnyak, Y. K. (2007) Mechanism and uses of a membrane peptide that targets tumors and other acidic tissues in vivo Proc. Natl. Acad. Sci. U. S. A. 104, 7893-7898, DOI: 10.1073/pnas.0702439104 
7. Viola-Villegas, N., Divilov, V., Andreev, O., Reshetnyak, Y., and Lewis, J. (2012) Towards the improvement of an acidosis-targeting peptide PET tracer J. Nucl. Med. 53, 1673

8. Yao, L., Daniels, J., Wijesinghe, D., Andreev, O. A., and Reshetnyak, Y.K. (2013) PHLIP®-mediated delivery of PEGylated liposomes to cancer cells $\mathrm{J}$. Controlled Release 167, 228-237, DOI: 10.1016/j.jconrel.2013.01.037

9. Yao, L., Daniels, J., Moshnikova, A., Kuznetsov, S., Ahmed, A., Engelman, D.M., Reshetnyak, Y. K., andAndreev, O. A. (2013) pHLIP peptide targets nanogold particles to tumors Proc. Natl. Acad. Sci. U. S. A.110, 465-470, DOI: 10.1073/pnas.1219665110

10. Vavere, A.L., Biddlecombe, G.B., Spees, W.M., Garbow, J.R., Wijesinghe, D., Andreev, O. A., Engelman, D. M., Reshetnyak, Y. K., and Lewis, J. S. (2009) A novel technology for the imaging of acidic prostate tumors by positron emission tomography Cancer Res. 69, 4510-4516, DOI: 10.1158/0008-5472.CAN-08-3781

11. Daumar, P., Wanger-Baumann, C. A., Pillarsetty, N., Fabrizio, L., Carlin, S. D., Andreev, O. A., Reshetnyak, Y. K., and Lewis, J. S. (2012) Efficient ${ }^{18}$ F-labeling of large 37-amino-acid pHLIP peptide analogues and their biological evaluation Bioconjugate Chem. 23, 1557-1566, DOI: 10.1021/bc3000222

12. Viola-Villegas, N. T., Carlin, S. D., Ackerstaff, E., Sevak, K. K., Divilov, V., Serganova, I., Kruchevsky, N., Anderson, M., Blasberg, R. G., and Andreev, O. A. (2014) Understanding the pharmacological properties of a metabolic PET tracer in prostate cancer Proc. Natl. Acad. Sci. U. S. A. 111, 7254-7259, DOI: 10.1073/pnas.1405240111 
13. Carroll, V., Demoin, D. W., Hoffman, T. J., and Jurisson, S. S. (2012) Inorganic chemistry in nuclear imaging and radiotherapy: current and future directions Radiochim. Acta 100, 653-667, DOI: 10.1524/ract.2012.1964

14. Zeglis, B. M., Houghton, J. L., Evans, M. J., Viola-Villegas, N., and Lewis, J. S. (2014) Underscoring the Influence of Inorganic Chemistry on Nuclear Imaging with Radiometals Inorg. Chem. 53, 1880-1899, DOI: 10.1021/ic401607z

15. McBride, W. J., D’Souza, C. $\quad$ A., Sharkey, R. M., Karacay, H., Rossi, E. A., Chang, C.-H., and Goldenberg, D. M. (2010) Improved ${ }^{18}$ F labeling of peptides with a fluoride-aluminum-chelate complex Bioconjugate Chem. 21, 1331-1340, DOI: $10.1021 / \mathrm{bc} 100137 \mathrm{x}$

16. McBride, W. J., Sharkey, R. M., Karacay, H., D’Souza, C. $\quad$ A., Rossi, E. A., Laverman, P., Chang, C.-H., Boerman, O. C., and Goldenberg, D. M. (2009) A Novel Method of ${ }^{18} F$ Radiolabeling for PET J. Nucl. Med. 50, 991-998, DOI: 10.2967/jnumed.108.060418

17. Weerakkody, D., Moshnikova, A., Thakur, M. S., Moshnikova, V., Daniels, J., Engelman, D. M., Andreev, O. A., and Reshetnyak, Y. K. (2013) Family of pH (low) insertion peptides for tumor targeting Proc. Natl. Acad. Sci. U. S. A. 110, 5834-5839, DOI: 10.1073/pnas.1303708110

18. Serganova, I., Rizwan, A., Ni, X., Thakur, S.

B., Vider, J., Russell, J., Blasberg, R., and Koutcher, J. A. (2011) Metabolic imaging: A link between lactate dehydrogenase A, lactate, and tumor phenotype Clin. Cancer Res. 17, 6250-6261, DOI: 10.1158/1078-0432.CCR-110397 
19. Tapmeier, T. T., Moshnikova, A., Beech, J., Allen, D., Kinchesh, P., Smart, S., Harris, A., McIntyre, A., Engelman, D. M., and Andreev, O. A. (2015), The pH low insertion peptide pHLIP variant 3 as a novel marker of acidic malignant lesions Proc. Natl. Acad. Sci. U. S. A. 112, 9710-9715, DOI: 10.1073/pnas. 1509488112

20. Bass, L. A., Wang, M., Welch, M. J., and Anderson, C. J. (2000) In Vivo Transchelation of Copper-64 from TETA-Octreotide to Superoxide Dismutase in Rat Liver Bioconjugate Chem. 11, 527-532, DOI: 10.1021/bc9901671

21. Zeglis, B. M., Sevak, K. K., Reiner, T., Mohindra, P., Carlin, S. D., Zanzonico, P., Weissleder, R., and Lewis, J. S. (2013) A Pretargeted PET Imaging Strategy Based on Bioorthogonal Diels-Alder Click Chemistry J. Nucl. Med. 54, 13891396, DOI: 10.2967/jnumed.112.115840

22. Dijkgraaf, I., Franssen, G. M., McBride, W. J., D’Souza, C. A., Laverman, P., Smith, C. J., Goldenberg, D. M., Oyen, W. J. G., and Boerman, O. C. (2012) PET of Tumors Expressing Gastrin-Releasing Peptide Receptor with an ${ }^{18}$ F-Labeled Bombesin Analog J. Nucl. Med. 53, 947-952, DOI: 10.2967/jnumed.111.100891 


\section{FIGURES}

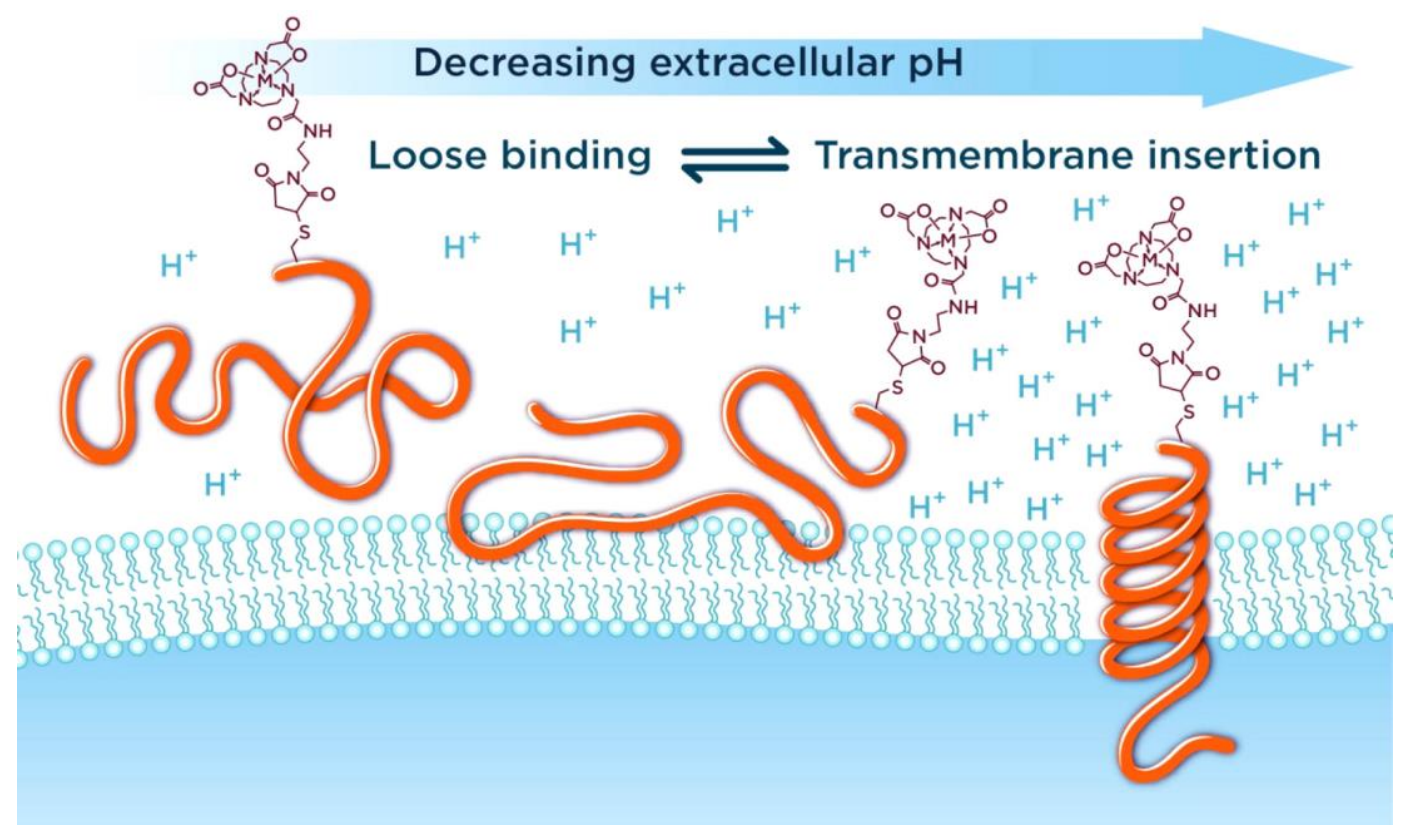

Figure 1. Three folding states of pHLIP peptides in decreasing extracellular $\mathrm{pH}$ : unfolded and unbound peptide in solution (State I), unfolded peptide loosely interacting with the membrane lipid bilayer at physiological $\mathrm{pH}$ (State II), and folded peptide in $\alpha$-helical conformation inserted across the membrane at low extracellular $\mathrm{pH}$ (State III). 


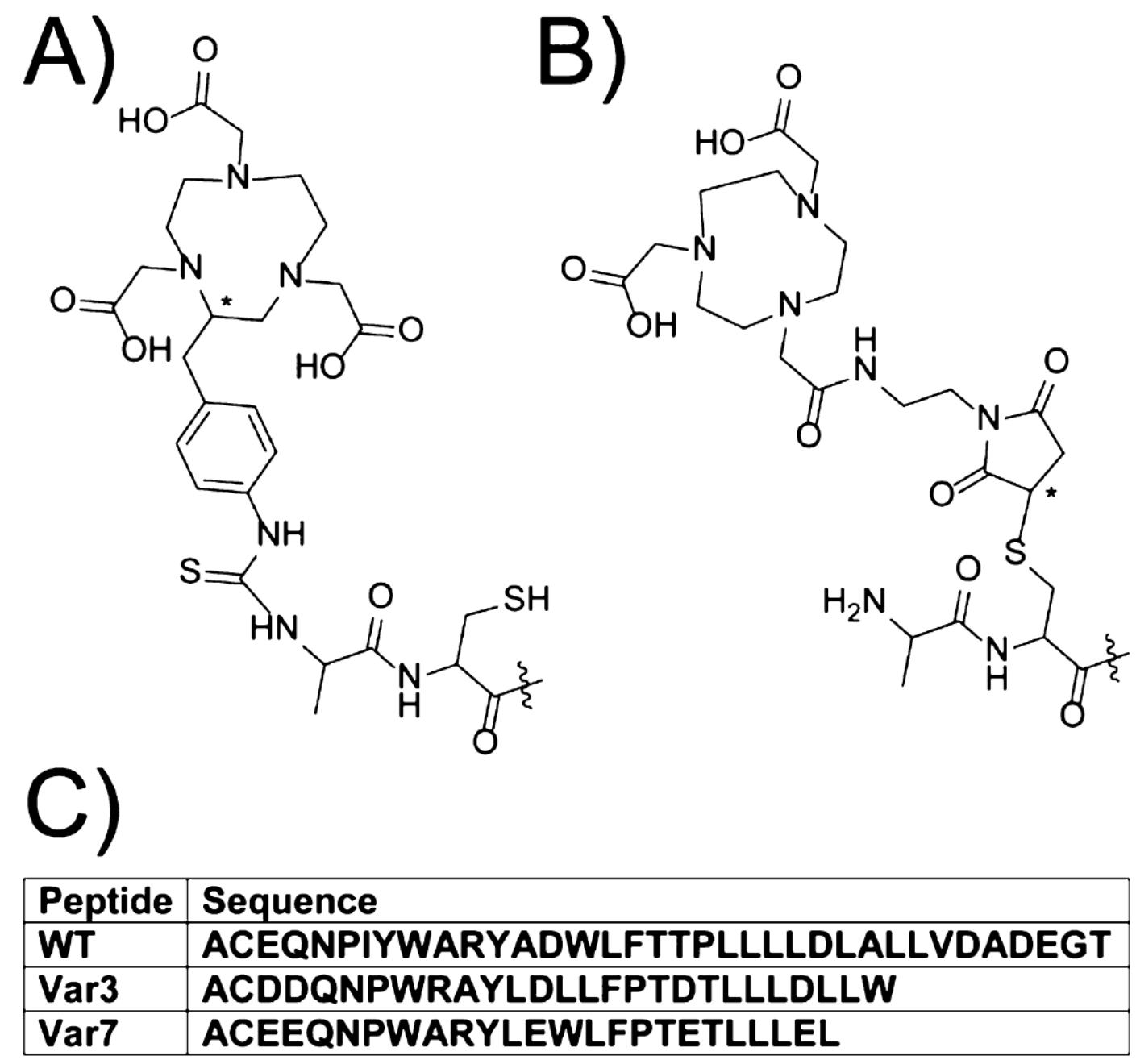

Figure 2. Two chelators [(A) NOTA-pHLIP and (B) NO2A-cyspHLIP] and (C) the peptide sequences used in this study. The chiral centers within the chelator and linker are marked with asterisks. 


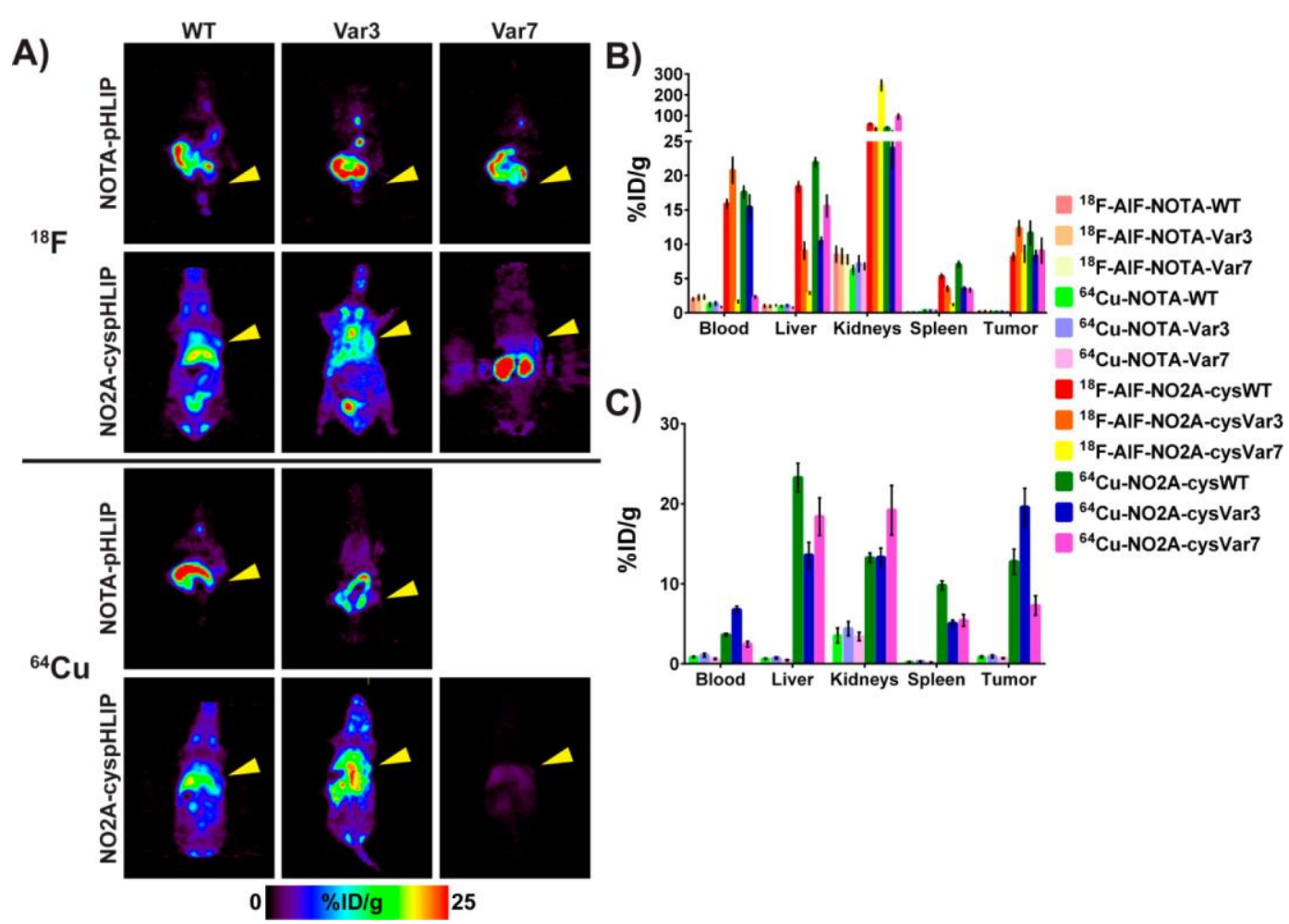

Figure 3. Coronal PET image slices at the level of the tumor at $4 \mathrm{~h}$ p.i. (A) and ex vivo biodistributions in selected organs at $4 \mathrm{~h}$ (B) and $24 \mathrm{~h}$ p.i. (C) showing differences in the tracer distribution in BALB/c female mice bearing 4T1 tumor allografts. The yellow arrowheads in (A) indicate where the tumor is located in the mouse. The selected scale on the PET images does not allow for tumor visualization in the case of the NO2A-cysVar7 constructs. Figure S2 shows the maximum intensity projections (MIPs) for these compounds at $4 \mathrm{~h}$ and the slices at the level of the tumor for the ${ }^{18} \mathrm{~F}-\mathrm{AlF}-\mathrm{NO} 2 \mathrm{~A}$-cyspHLIP constructs with a maximum value of $15 \% \mathrm{ID} / \mathrm{g}$. Only ex vivo biodistribution data was obtained for ${ }^{64} \mathrm{Cu}-\mathrm{NOTA}-\mathrm{Var} 7$. 


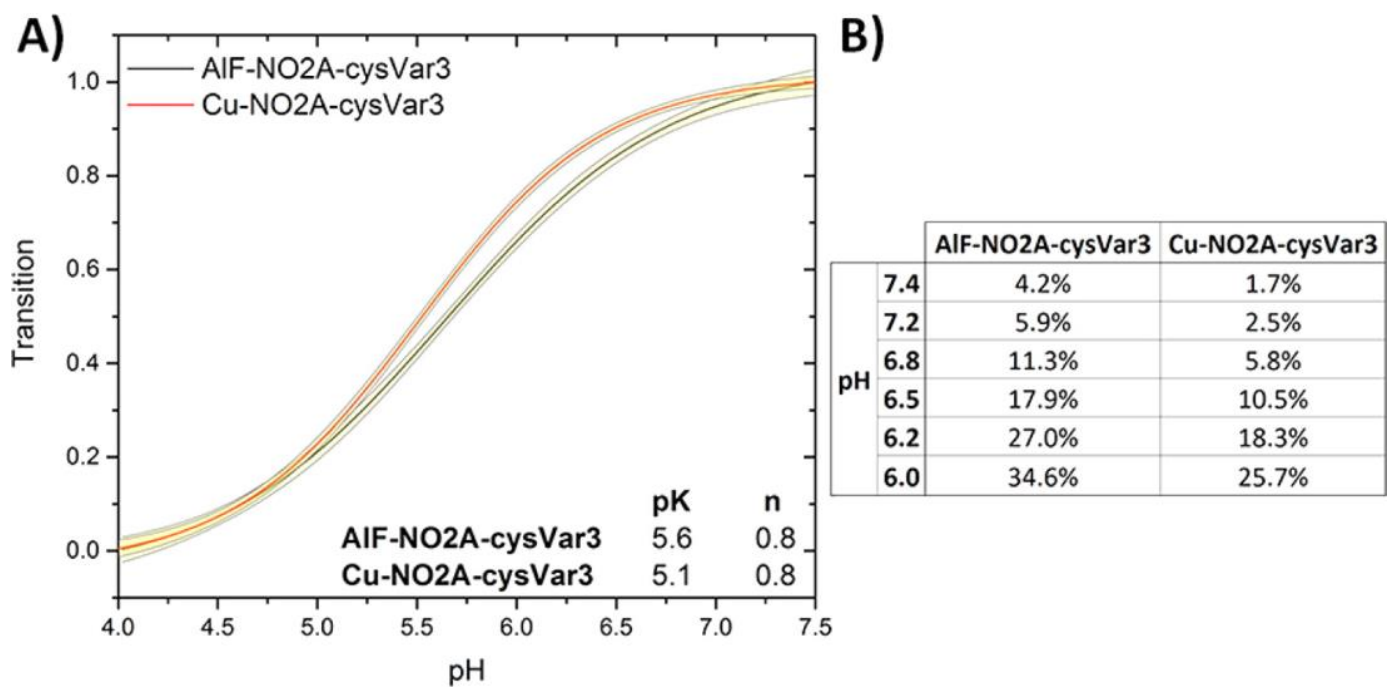

Figure 4. $\mathrm{pH}$ dependent bilayer insertion of NO2A-cysVar3 constructs. Changes in intrinsic construct fluorescence are used to measure the insertion of the construct population as a function of $\mathrm{pH}$ (transition from State II, at high $\mathrm{pH}$, to State III, at low $\mathrm{pH}$ ). Amount of construct population in State II is measured on the y-axis. 95\% confidence intervals are indicated by yellow bands (A). $\mathrm{pH}$-dependence parameters are used to calculate the percent of inserted construct population at various $\mathrm{pH}$ levels (B). These transitions were measured in the presence of physiological levels of free magnesium and calcium ions $(0.65 \mathrm{mM}$ and $1.25 \mathrm{mM}$, respectively). 


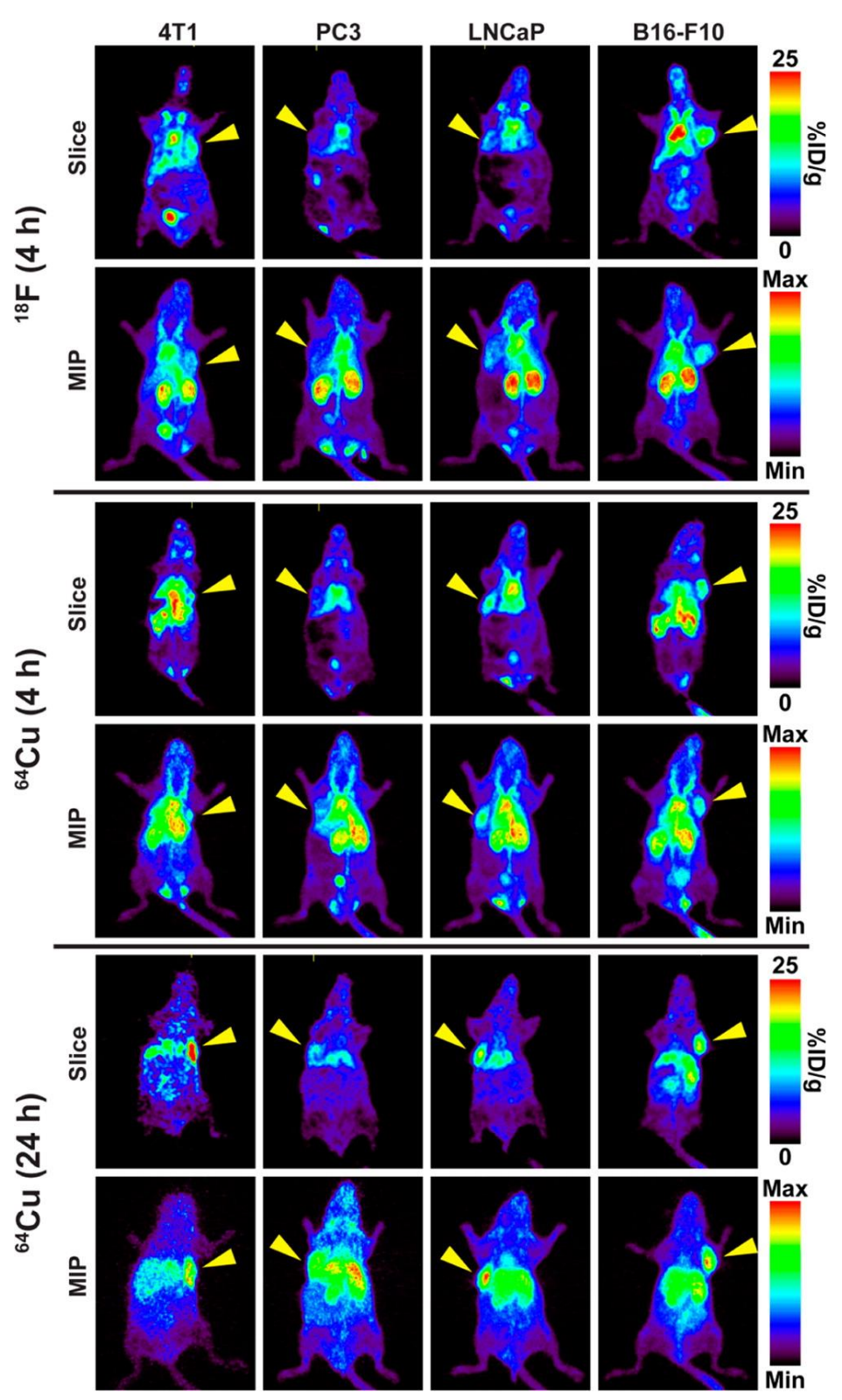

Figure 5. Slices and maximum intensity projections (MIP) from PET images showing differences in the radiolabeled NO2A-cysVar3 distribution at 4 and $24 \mathrm{~h}$ in BALB/c female mice bearing orthotopic 4T1 tumor allografts, nude male mice bearing shoulder PC3 or LNCaP xenografts, and C57Bl/6 female mice bearing orthotopic B16-F10 tumor allografts. The yellow arrowheads indicate where the tumor is located in the mouse. 


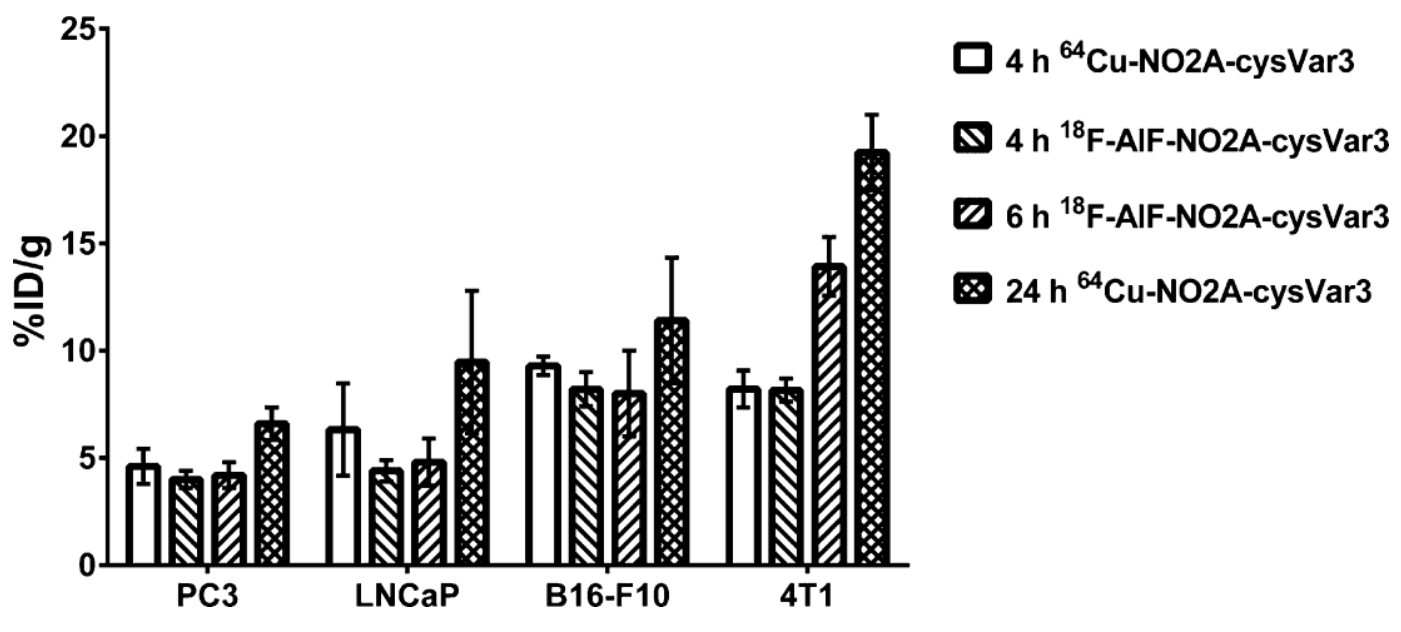

Figure 6. Graph of the ex vivo tumor uptake of the lead radiolabeled NO2A-cysVar3 in various tumor models at 4,6 , and $24 \mathrm{~h}$ p.i. 

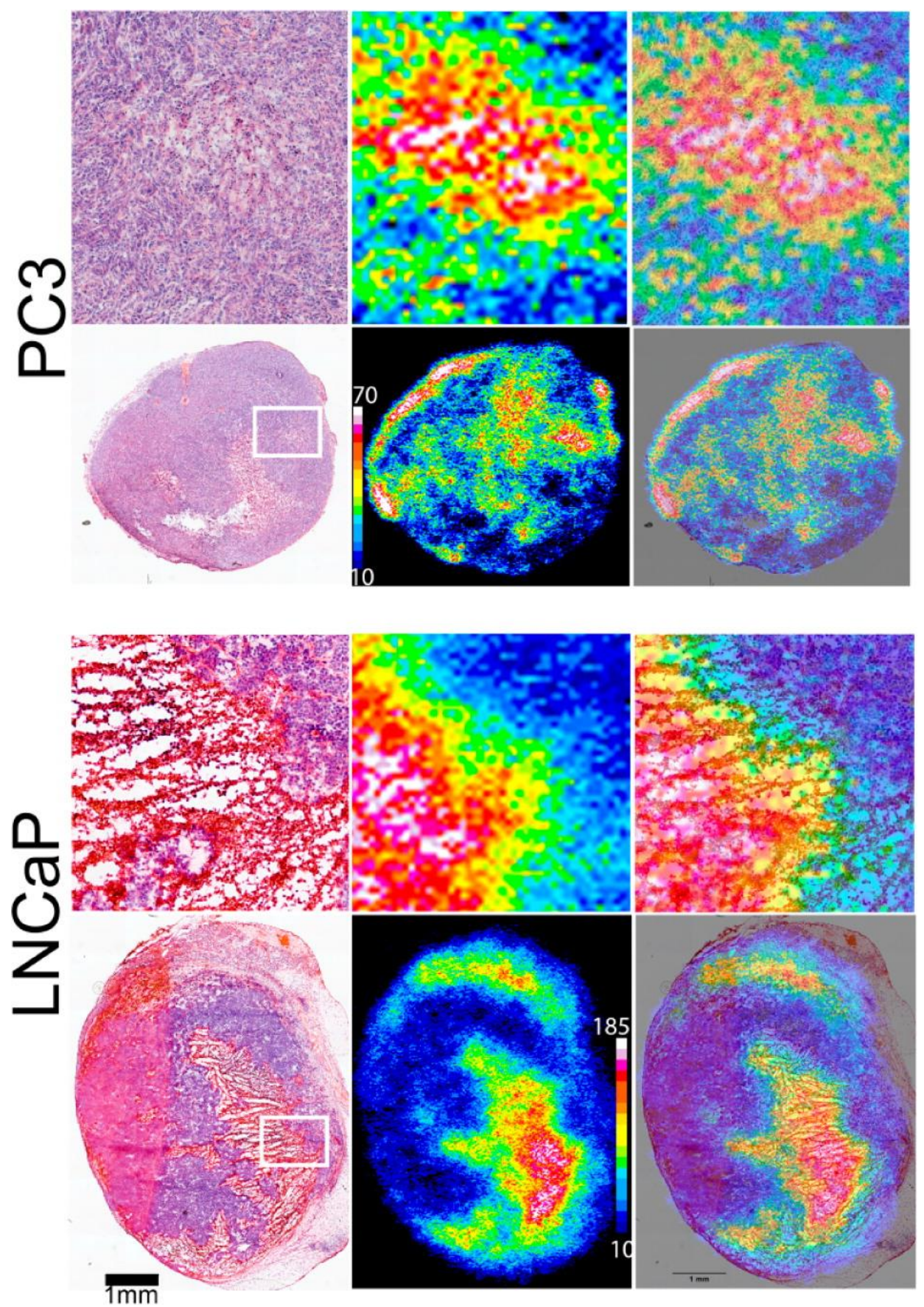

Figure 7. Comparison of uptake in sections of excised tumors from PC3 or LNCaP tumor-bearing male nude mice. The top panels are expansions of the whole tumor sections shown below. The left-most panels are H\&E stained, the middle panels are the autoradiography, and the right-most panels are overlays. 


\section{TABLES}

\begin{tabular}{|c|c|c|c|c|c|c|}
\hline tissue & $\begin{array}{c}{ }^{64} \mathrm{Cu}-\mathrm{NOTA}- \\
\text { WT }(n=4)\end{array}$ & $\begin{array}{l}{ }^{64} \mathrm{Cu}-\mathrm{NOTA}- \\
\operatorname{Var} 3(n=5)\end{array}$ & $\begin{array}{l}{ }^{64} \mathrm{Cu}-\mathrm{NOTA}- \\
\operatorname{Var7}(n=4)\end{array}$ & $\begin{array}{c}{ }^{18} \mathrm{~F}-A I F- \\
\text { NOTA-WT } \\
(n=5)\end{array}$ & $\begin{array}{c}{ }^{18} \mathrm{~F}-A \mathrm{AIF}- \\
\text { NOTA-Var3 } \\
(n=4)\end{array}$ & $\begin{array}{c}{ }^{18} \mathrm{~F}-A \mathrm{AIF}- \\
\text { NOTA-Var7 } \\
(n=5)\end{array}$ \\
\hline lood & 1. & & 8 & & & \\
\hline & 0.50 & 0.49 & 0.35 & $0.66 \pm$ & $0.78 \pm$ & 0.78 \\
\hline & & 31.4 & & & & \\
\hline & & & & & & \\
\hline & & & & & & \\
\hline & & & & & & 00. \\
\hline & & & & & 20 & 30.5 \\
\hline $\begin{array}{l}\text { s. } \\
\text { intest }\end{array}$ & 0. & & & 20 & 927 & 20.77 \\
\hline I. & 9.9 & 7.4 & 7.7 & 35 & & 22. \\
\hline & 6.2 & & & & & \\
\hline & 0.16 & & & & & \\
\hline & 0.27 & & & & & \\
\hline & 0.7 & & & & & \\
\hline & & & & & & \\
\hline & & & & & & \\
\hline
\end{tabular}

Table 1. Biodistribution $(\% \mathrm{ID} / \mathrm{g})$ of the Six Radiolabeled N-Terminus Derivatized

NOTA-pHLIP Derivatives in 4T1 Allografted Female BALB/c Mice at 4 h p.i. 


\begin{tabular}{|c|c|c|c|c|c|c|}
\hline tissue & $\begin{array}{l}{ }^{64} \mathrm{Cu}- \\
\text { NO2A- } \\
\text { cysWT } \\
(n=4)\end{array}$ & $\begin{array}{c}{ }^{64} \mathrm{Cu}- \\
\text { NO2A- } \\
\text { cysVar3 } \\
(n=5)\end{array}$ & $\begin{array}{c}{ }^{64} \mathrm{Cu}- \\
\text { NO2A- } \\
\text { cysVar7 } \\
(n=4)\end{array}$ & $\begin{array}{l}{ }^{18} \text { F-AIF- } \\
\text { NO2A- } \\
\text { cysWT } \\
(n=4)\end{array}$ & $\begin{array}{l}{ }^{18} \text { F-AIF- } \\
\text { NO2A- } \\
\text { cysVar3 } \\
(n=10)\end{array}$ & $\begin{array}{c}{ }^{18} \mathrm{~F}-\mathrm{AlF}- \\
\text { NO2A- } \\
\text { cysVar7 } \\
(n=4)\end{array}$ \\
\hline blood & $17.6 \pm 0.86$ & $15.4 \pm 1.76$ & $2.31 \pm 0.18$ & $15.9 \pm 0.63$ & $20.8 \pm 1.88$ & $1.64 \pm 0.18$ \\
\hline heart & $5.52 \pm$ & $5.12+0.63$ & 2.68 & $4.78 \pm 0.53$ & $6.29 \pm 1.03$ & $0.89 \pm 0.12$ \\
\hline lungs & $12.3 \pm$ & $.7 \pm$ & $4.21 \pm$ & $8.73 \pm 0.84$ & $11.8 \pm 3.79$ & $1.66 \pm 0.07$ \\
\hline liver & $21.9 \pm$ & $10.4 \pm 0$ & $15.6 \pm$ & $12.6 \pm 11.4$ & $9.06 \pm 1.24$ & $2.90 \pm 0.18$ \\
\hline spleen & $7.07 \pm c$ & $3.45 \pm 0$ & $3.31 \pm 0.24$ & $5.31 \pm 0.34$ & $3.73 \pm 0.66$ & $1.19 \pm 0.10$ \\
\hline pancreas & $3.00 \pm$ & $2.31 \pm 0$ & 1.8 & $2.10 \pm 0.43$ & $2.44 \pm 0.20$ & $0.49 \pm 0.03$ \\
\hline stom & $1.82 \pm$ & & 3.33 & $1.31 \pm 0.96$ & $1.29 \pm 0.62$ & $0.68 \pm 0.10$ \\
\hline s. inte & $4.56 \pm$ & م & $6.37 \pm$ & $2.85 \pm 0.31$ & $2.06 \pm 0.28$ & $0.91 \pm 0.03$ \\
\hline I. intestine & $4.09 \pm$ & $109+0$ & $8.19 \pm 0.85$ & $1.47 \pm 0.14$ & $2.07 \pm 0.63$ & $0.91 \pm 0.12$ \\
\hline kidneys & $40.6 \pm 4.0$ & $24.0 \pm 2.7$ & $95.5 \pm 11.9$ & $59.1 \pm 3.63$ & $34.3 \pm 6.91$ & $246 \pm 25.6$ \\
\hline muscle & $1.15 \pm$ & . & 0.6 & $1.23 \pm 0.17$ & $1.55 \pm 0.27$ & $0.35 \pm 0.06$ \\
\hline bone & $3.04 \pm c$ & \pm 0 & \pm 0 & $1.94 \pm 0.45$ & $1.66 \pm 0.61$ & $0.60 \pm 0.12$ \\
\hline skin & $3.23 \pm 0.12$ & $3.43 \pm 0.34$ & $2.59 \pm 0.05$ & $2.85 \pm 0.22$ & $2.92 \pm 0.71$ & $1.59 \pm 0.10$ \\
\hline brain & $0.62 \pm 0$ & $0.35 \pm 0$ & $0.25 \pm 0.02$ & $0.34 \pm 0.06$ & $0.52 \pm 0.14$ & $0.05 \pm 0.01$ \\
\hline tumor & $11.7 \pm 1.7$ & $8.21 \pm 0$ & $9.07 \pm 1.81$ & $8.16 \pm 0.53$ & $10.6 \pm 2.26$ & $8.61 \pm 1.21$ \\
\hline
\end{tabular}

Table 2. Biodistribution (\% ID/g) of the Six Radiolabeled Cysteine Derivatized NO2A-cyspHLIP Derivatives in 4T1 Allografted Female BALB/c Mice at 4 h p.i. 


\section{SUPPORTING INFORMATION}

\section{METHODS \& MATERIALS}

\section{Radiolabeling}

Labeling methods for ${ }^{64} \mathrm{Cu}$-NOTA-pHLIP. Following a preparation similar to Zeglis et al., ${ }^{1} 3.13-11.26 \mathrm{mCi}(116-417 \mathrm{MBq})$ of ${ }^{64} \mathrm{Cu}$ in $0.1 \mathrm{M} \mathrm{HCl}(2.5-6.0 \mu \mathrm{L}, 1.86 \times 104-$ $1.25 \times 104 \mathrm{mCi} / \mu \mathrm{mol}$, Washington University, St. Louis, MO) was added to $150 \mu \mathrm{L}$ of $100 \mathrm{mM} \mathrm{NH} \mathrm{NHAc}_{4}$ buffer $(\mathrm{pH} \sim 5.5)$. An aliquot $(25-37 \mu \mathrm{L})$ of NOTA-pHLIP derivative (0.46-0.63 mM) in DMSO was added to the $\mathrm{pH}$ adjusted ${ }^{64} \mathrm{Cu}$ solution. The solution was placed on a thermomixer (Eppendorf, Hamberg, Germany) at $80{ }^{\circ} \mathrm{C}$ for $15 \mathrm{~min}$ at $1100 \mathrm{rpm}$. An aliquot of the crude product mixture was HPLC-analyzed (5$95 \%$ acetonitrile in water (with $0.1 \%$ trifluoroacetic acid) over $15 \mathrm{~min}$, Jupiter C-18 column, Phenomenex, $250 \mathrm{~mm} \times 4.6 \mathrm{~mm}, 5 \mu \mathrm{m}, 300 \AA$ ) to ensure that product was present $\left({ }^{64} \mathrm{Cu}-\mathrm{NOTA}-\mathrm{pHLIP}\right.$ eluted at $10.3 \mathrm{~min} ;{ }^{64} \mathrm{Cu}-\mathrm{NO} 2 \mathrm{~A}-\mathrm{cyspHLIP}$ eluted at 13.1 min). The remainder of the reaction mixture was separated using a pretreated Oasis HLB Plus Light or C18 Sep-Pak Light cartridge (Waters, Milford, MA). Free and unbound ${ }^{64} \mathrm{Cu}$ was removed by washing the cartridge with $5 \mathrm{~mL}$ of water. The pure ${ }^{64} \mathrm{Cu}$-labeled NOTA-pHLIP derivatives were then eluted with $1 \mathrm{~mL}$ ethanol in $0.1 \mathrm{~mL}$ fractions. The ethanolic fractions with the highest activities were combined and diluted with sterile phosphate buffered saline (PBS) to provide $<10 \%$ ethanol in the solution before HPLC analysis (Jupiter C-18, $5 \mu \mathrm{m}, 300 \AA ⿻, 250 \times 4.6 \mathrm{~mm}$, Phenomenex; 5-95\% AcN in water with $0.1 \%$ TFA over $15 \mathrm{~min}$ at $1 \mathrm{~mL} / \mathrm{min}$ ) to

ensure that no free ${ }^{64} \mathrm{Cu}$ was present. The radiochemical purity of the species was always $>93 \%$ and the specific activity (using the original amount of peptide added to 
the reaction mixture) was estimated to be $56.0-318.4 \mu \mathrm{Ci} / \mathrm{nmol}(3.05-11.8 \mathrm{GBq} / \mu \mathrm{mol})$. The reaction yielded 1.29-9.54 mCi (47.7-353 MBq) of product with an isolated yield of $40.7-84.7 \%$.

Labeling methods for ${ }^{18}$ F-AlF-NOTA-pHLIP. Following a modified labeling method described by Dijkgraaf et al., ${ }^{2} 52.0-60.8 \mathrm{mCi}(1.92-2.25 \mathrm{GBq})$ of ${ }^{18} \mathrm{~F}$ target water (produced from ${ }^{18} \mathrm{O}$ enriched target water using a GEMS PETtrace-800 cyclotron, Memorial Sloan Kettering Cancer Center, New York, NY) was obtained. The target water was loaded onto a preconditioned chromafix cartridge (30-PS-HCO3, Advanced Biochemical Compounds (ABX), Germany). [All of the preconditioning solvents and the elution solution were prepared with metal-free water and trace-metal free reagents.] The ${ }^{18} \mathrm{~F}$ was eluted from the cartridge with approximately $100 \mu \mathrm{L}$ of $0.4 \mathrm{M}$ KHCO3 in metal-free water into a $1.5 \mathrm{~mL}$ tube (ThermoFisher Scientific, Waltham, MA). The $\mathrm{pH}$ was adjusted to 4.1 with metal-free acetic acid (Sigma Aldrich). To the $\mathrm{pH}$ adjusted solution, $100 \mu \mathrm{L}$ of acetonitrile was added. If the total activity exceeded $50 \mathrm{mCi}(1.85 \mathrm{GBq})$, the mixture was split between multiple $1.5 \mathrm{~mL}$ tubes prior to continuing ( $\sim 10 \mathrm{mCi} / 40 \mathrm{nmol}$ reaction). To the aliquotted mixture, $20 \mu \mathrm{L}(40 \mathrm{nmol})$ of $2 \mathrm{mM} \mathrm{AlCl}_{3}$ in $0.1 \mathrm{M} \mathrm{NH4OAc}$ buffer $(\mathrm{pH} \sim 4.1)$ was added to each tube. The solution was allowed to react for $5 \mathrm{~min}$ at room temperature before $14-17 \mu \mathrm{L}$ of $4-5 \mathrm{mM}$ NOTA-peptide in DMSO (68-75 nmol) was added, the tube capped, and the reaction mixture reacted at $75{ }^{\circ} \mathrm{C}$ for 15 minutes in a thermomixer. After reaction, the mixture was diluted with $2 \mathrm{~mL}$ of metal-free water. An aliquot of the reaction mixture was HPLC-analyzed (20-95\% acetonitrile in water (with $0.1 \%$ trifluoroacetic acid) over 15 min with an Atlantis T3, Waters, $250 \mathrm{~mm} \times 4.6 \mathrm{~mm}, 5 \mu \mathrm{m}$; Jupiter, Phenomenex, 
$250 \mathrm{~mm} \times 4.6 \mathrm{~mm}, 5 \mu \mathrm{m}, 300 \AA$, column $)$ to ensure that product was formed $\left({ }^{18} \mathrm{~F}\right.$-AlFNOTA-WT eluted between 10.1 and $12.1 \mathrm{~min} ;{ }^{18} \mathrm{~F}$-AlF-NOTA-var3 eluted between 10.2 and $11.4 \mathrm{~min} ;{ }^{18} \mathrm{~F}$-AlF-NOTA-var7 eluted between 8.2 and $10.3 \mathrm{~min} ;{ }^{18} \mathrm{~F}$-AlFNOTA-cysWT eluted between 13 and $16 \mathrm{~min} ;{ }^{18} \mathrm{~F}$-AlF-NOTA-cysVar3 eluted between 12 and $15 \mathrm{~min} ;{ }^{18}$ F-AlF-NOTA-cysVar7 eluted between 12 and $14 \mathrm{~min}$ ). While the HPLC analysis was being performed, the remainder of the reaction mixture was separated using a pretreated Oasis HLB Plus Light or C18 Sep-Pak Light cartridge (Waters, Milford, MA) eluting first with water $(5 \mathrm{~mL})$ to remove any unbound ${ }^{18} \mathrm{~F}$ species and then ethanol $(0.1 \mathrm{~mL}$ fractions for 4 fractions and then 0.6 $\mathrm{mL})$. The ${ }^{18}$ F-AlF-NOTA-pHLIP derivatives eluted within the first three fractions in ethanol. The ethanolic solution was diluted with sterile PBS to provide $<10 \%$ ethanol in the solution before HPLC analysis (Jupiter C-18, $5 \mu \mathrm{m}, 300 \AA, 250 \times 4.6 \mathrm{~mm}$, Phenomenex; 5-95\% AcN in water with $0.1 \%$ TFA over $15 \mathrm{~min}$ at $1 \mathrm{~mL} / \mathrm{min})$. The radiochemical purity of the species was always $\geq 95 \%$ and the specific activity (using the original amount of peptide added to the reaction mixture) was estimated to be 79.9-178.1 $\mu \mathrm{Ci} / \mu \mathrm{mol}(2.96-6.60 \mathrm{GBq} / \mu \mathrm{mol})$. The reaction yielded $6.02-9.24 \mathrm{mCi}$ (223-342 MBq) with an overall, decay-corrected (to beginning of labeling procedure) isolated yield of $2.33-48.6 \%$.

\section{Nonradioactive standards}

General labeling methods for Cu-NOTA-pHLIP. Approximately $200 \mu \mathrm{L}$ of $36.4 \mathrm{M}$ $\mathrm{Cu}(\mathrm{OAc})_{2}$ in $0.1 \mathrm{M} \mathrm{NH}_{4} \mathrm{OAc}(\mathrm{pH} \sim 5)$ in metal-free water was added to 5-6 $\mathrm{mg}$ of peptide in approximately $200 \mu \mathrm{L}$ of DMSO. The mixtures were reacted in a 
thermomixer at $50{ }^{\circ} \mathrm{C}$ for $1 \mathrm{~h}$, and then filtered through a $0.2 \mu \mathrm{m}$ filter. The filter was washed with an additional $1 \mathrm{~mL}$ of $50 \% \mathrm{AcN}$ in water and $2 \mathrm{~mL}$ of water, sequentially. The combined filtrate was HPLC purified (10-95\% AcN in water over 30 min with a Jupiter C-18 column, $5 \mu \mathrm{m}, 300 \AA, 250 \times 4.6 \mathrm{~mm}$, Phenomenex). The collected fraction (14.5-15.5 min for Cu-NOTA-WT, 13.9-15 min for Cu-NOTAVar3, 13.2-14.1 min for Cu-NOTA-Var7, 12.2-13.5 min for Cu-NO2A-cysWT, 1315.3 min for $\mathrm{Cu}-\mathrm{NO} 2 \mathrm{~A}-\mathrm{cys} V a r 3$, and 12.5-16 min $\mathrm{Cu}-\mathrm{NO} 2 \mathrm{~A}-\mathrm{cys} V \operatorname{ar} 7$ ) was lyophilized.

General labeling methods for AlF-NOTA-pHLIP. Sodium fluoride (19.8 mg, 0.47 mmol) was dissolved in $150 \mu \mathrm{L}$ of $0.1 \mathrm{M} \mathrm{NH}_{4} \mathrm{OAc}$ in metal-free water $(\mathrm{pH} \sim 4.1)$. The resulting solution was added to $13.7 \mathrm{mg} \mathrm{AlCl}_{3}(0.057 \mathrm{mmol})$ with $50 \mu \mathrm{L}$ of $0.1 \mathrm{M}$ $\mathrm{NH}_{4} \mathrm{OAc}$ buffer $(\mathrm{pH} \sim 4.1)$. The $\mathrm{AlF}_{3}$ solution $(60 \mu \mathrm{L})$ was added to approximately 7 mg of peptide dissolved in $60 \mu \mathrm{L}$ of $\mathrm{EtOH}$. The reaction mixtures were reacted at 50 ${ }^{\circ} \mathrm{C}$ on a thermomixer for $1 \mathrm{~h}$ before being HPLC purified (10-95\% AcN in water over 30 min with a Jupiter column, $5 \mu \mathrm{m}, 300 \AA, 250 \times 4.6 \mathrm{~mm}$, Phenomenex). The collected fraction (14.7-16.5 min for AlF-NOTA-WT, 13.8-15.4 min for AlF-NOTAVar3, 13-14.6 min for AlF-NOTA-Var7, 12.5-17.5 min for AlF-NO2A-cysWT and AlF-NO2A-cysVar3, and 12-16 min AlF-NO2A-cysVar7) was lyophilized.

\section{Cell culture methods and in vivo models}

$4 T 1$ cell culture. The $4 \mathrm{~T} 1$ cells, derived from spontaneous breast tumor in a BALB/c mouse, were provided by Fred Miller (Karmanos Cancer Institute, Detroit, MI) and cultured in Dulbecco's modified Eagle's high glucose media with 10 \% FCS, 2 mM L- 
glutamine, penicillin, and streptomycin or from ATCC (Manassas, VA) and cultured in RPMI-1640 medium modified to contain $2 \mathrm{mM}$ L-glutamine, $10 \mathrm{mM}$ HEPES, 1 $\mathrm{mM}$ sodium pyruvate, $4.5 \mathrm{~g} / \mathrm{L}$ glucose, $1.5 \mathrm{~g} / \mathrm{L} \mathrm{NaHCO3}$, penicillin, and streptomycin.

$4 T 1$ orthotopic BALB/c mouse model. The cells were removed from the flasks, concentrated, and resuspended in minimal media for cell counting. The cells were then diluted to contain approximately 1 million cells in $30 \mu \mathrm{L}$ of media (for orthotopic allografts). Following an MSKCC Institutional Animal Care and Use Committee approved protocol, 8-10-week-old BALB/c mice (Charles River Laboratories, Wilmington, MA) were surgically implanted with one million 4T1 cancer cells into the first or the last mammary fat pad of the right side of the animal using aseptic surgical techniques and sterile staple closures while the mice were under anesthetic. Additionally, mice were given injections of meloxicam ( $24 \mathrm{~h}$ pain killer) in the scruff and bupivacaine intradermally prior to surgical incisions. One day post-surgery, the mice were again given meloxicam and checked to ensure that the closure was healing. Two and three days post-surgery, the mice were checked to ensure that the animals were healthy and recovering well from the surgery. Five to seven days post-surgery, the staples were removed. Five to nine days post-surgery, the mice were injected with 50-75 $\mu \mathrm{Ci}(0.9-0.4 \mathrm{nmol})$ of the respective radiopharmaceutical for in vivo biodistribution or 500-600 $\mu \mathrm{Ci}(9-11 \mathrm{nmol})$ for in vivo imaging. The mice receiving ${ }^{64} \mathrm{Cu}$-NOTA-pHLIP were sacrificed at $1,2,4,12,24,36$, and $48 \mathrm{~h}$; the mice receiving ${ }^{18}$ F-AlF-NOTA-pHLIP were sacrificed at $0.5,1,2,4,6$ and $8 \mathrm{~h}$. 
PC3 cell culture. The PC3 cells, prostate cancer derived from a human bone metastasis, were purchased from ATCC (Manassas, VA) and cultured in F-12K Medium (Kaighn's Modification of Ham's F-12 Medium) with, 10\% FCS, 2 mM Lglutamine, $1.5 \mathrm{~g} / \mathrm{L} \mathrm{NaHCO} 3$, penicillin, and streptomycin.

PC3 shoulder nude mouse xenografts. The cells were removed from the flasks, concentrated, and resuspended in minimal media for cell counting. The cells were then diluted to contain approximately 3 million cells in $150 \mu \mathrm{L}$ of 1:1 media and matrigel (for shoulder xenografts). Following an MSKCC Institutional Animal Care and Use Committee approved protocol, $150 \mu \mathrm{L}$ of the cell suspension was injected subcutaneously in 6-9-week-old nude male mice (CRL). Mice were used in studies at 3 weeks post inoculation. The mice were injected with $50-75 \mu \mathrm{Ci}(0.9-0.4 \mathrm{nmol})$ of the respective radiopharmaceutical for in vivo biodistribution or 500-600 $\mu \mathrm{Ci}(9-11 \mathrm{nmol})$ for in vivo imaging. The mice receiving ${ }^{64} \mathrm{Cu}-\mathrm{NOTA}$-cys Var3 were sacrificed at 1,4 , 12, 24, and $48 \mathrm{~h}$; the mice receiving ${ }^{18} \mathrm{~F}$-AlF-NOTA-cysVar3 were sacrificed at $1,4,6$ and $8 \mathrm{~h}$.

LNCaP cell culture. The $\mathrm{LNCaP}$ cells, prostate cancer derived from a human supraclavicular lymph node metastasis, were purchased from ATCC (Manassas, VA) and cultured in RPMI-1640 medium modified to contain $2 \mathrm{mM}$ L-glutamine, $10 \mathrm{mM}$ HEPES, $1 \mathrm{mM}$ sodium pyruvate, $4.5 \mathrm{~g} / \mathrm{L}$ glucose, and $1.5 \mathrm{~g} / \mathrm{L} \mathrm{NaHCO}_{3}$, penicillin, and streptomycin.

LNCaP shoulder nude mouse xenografts. The cells were removed from the flasks, concentrated, and resuspended in minimal media for cell counting. The cells were then diluted to contain approximately 3-6 million cells in $150 \mu \mathrm{L}$ of $1: 1$ media and matrigel 
(or shoulder xenografts). Following an MSKCC Institutional Animal Care and Use Committee approved protocol, $150 \mu \mathrm{L}$ of the cell suspension was injected subcutaneously in 6-8-week-old nude male mice (CRL). Mice were used in studies at 3-8 weeks post inoculation. The mice were injected with $50-75 \mu \mathrm{Ci}(0.9-0.4 \mathrm{nmol})$ of the respective radiopharmaceutical for in vivo biodistribution or $500-600 \mu \mathrm{Ci}(9-11$ nmol) for in vivo imaging. The mice receiving ${ }^{64} \mathrm{Cu}-\mathrm{NOTA}-\mathrm{cys} V$ ar 3 were sacrificed at $1,4,12,24$, and $48 \mathrm{~h}$; the mice receiving ${ }^{18} \mathrm{~F}$-AlF-NOTA-cys Var3 were sacrificed at 1 , 4, 6 and $8 \mathrm{~h}$.

B16-F10 cell culture. The B16-F10 cells, derived from spontaneous melanoma in a C57Bl/6 mouse, were purchased from ATCC (Manassas, VA) and cultured in Dubelco's modified Eagle's high glucose media with 10 \% FCS, 4 mM L-glutamine, 1 $\mathrm{mM}$ sodium pyruvate, $1.5 \mathrm{~g} / \mathrm{L} \mathrm{NaHCO}_{3}$, penicillin, and streptomycin.

Orthotopic B16-F10 shoulder allografts. The cells were removed from the flasks, concentrated, and resuspended in minimal media for cell counting. The cells were then diluted to contain approximately 100,000 cells in $100 \mu \mathrm{L}$ of media (for shoulder allografts). Following an MSKCC Institutional Animal Care and Use Committee approved protocol, $\mathrm{C} 57 \mathrm{Bl} / 6$ female mice $(\mathrm{CRL})$ and $\mathrm{BALB} / \mathrm{c}$ female mice $(\mathrm{CRL})$ were injected with $100 \mu \mathrm{L}$ of the cell suspension intradermally as per a media substituted protocol from Overwijk \& Restifo (Current Protocols in Immunology, 2000: 20.1.120.1.29). The mice were used in studies at approximately 9-14 days post inoculation. The mice were injected with $50-75 \mu \mathrm{Ci}(0.9-0.4 \mathrm{nmol})$ of the respective radiopharmaceutical for in vivo biodistribution or 500-600 $\mu \mathrm{Ci}(9-11 \mathrm{nmol})$ for in vivo imaging. The mice receiving ${ }^{64} \mathrm{Cu}$-NOTA-cysVar3 were sacrificed at $1,4,12,14.5$, 
and $24 \mathrm{~h}$; the mice receiving ${ }^{18} \mathrm{~F}$-AIF-NOTA-cysVar3 were sacrificed at 1, 4, 6 and 8 h.

U-87 MG cell culture. The human glioblastoma cell line, U-87 MG, was purchased from American Type Culture Collection (ATCC, Manassas, VA) and cultured in Dulbecco's Modified Eagle's Medium with $10 \%$ fetal bovine serum, $2 \mathrm{mM}$ LGlutamine, $1500 \mathrm{mg} / \mathrm{L}$ Sodium Bicarbonate, 100 units $/ \mathrm{mL}$ penicillin $\mathrm{G}$ and $100 \mu \mathrm{g} / \mathrm{mL}$ streptomycin (prepared by the MSKCC Media Preparation Core).

U-87 MG shoulder xenografts. The cells were removed from the flasks, concentrated, and resuspended in minimal media for cell counting. The cells were then diluted to contain approximately 1 million cells in $150 \mu \mathrm{L}$ of $1: 1$ media and matrigel (or shoulder xenografts). Following an MSKCC Institutional Animal Care and Use Committee approved protocol, $150 \mu \mathrm{L}$ of the cell suspension was injected subcutaneously in 12-week-old nude male mice (CRL). Mice were used in studies at 2-3 weeks post inoculation. The mice were injected with $400-500 \mu \mathrm{Ci}(7-10 \mathrm{nmol})$ for in vivo imaging. The mice were sacrificed at $6 \mathrm{~h}$ after imaging $(\mathrm{n}=6)$. 


\section{SUPPORTING INFORMATION FIGURES}

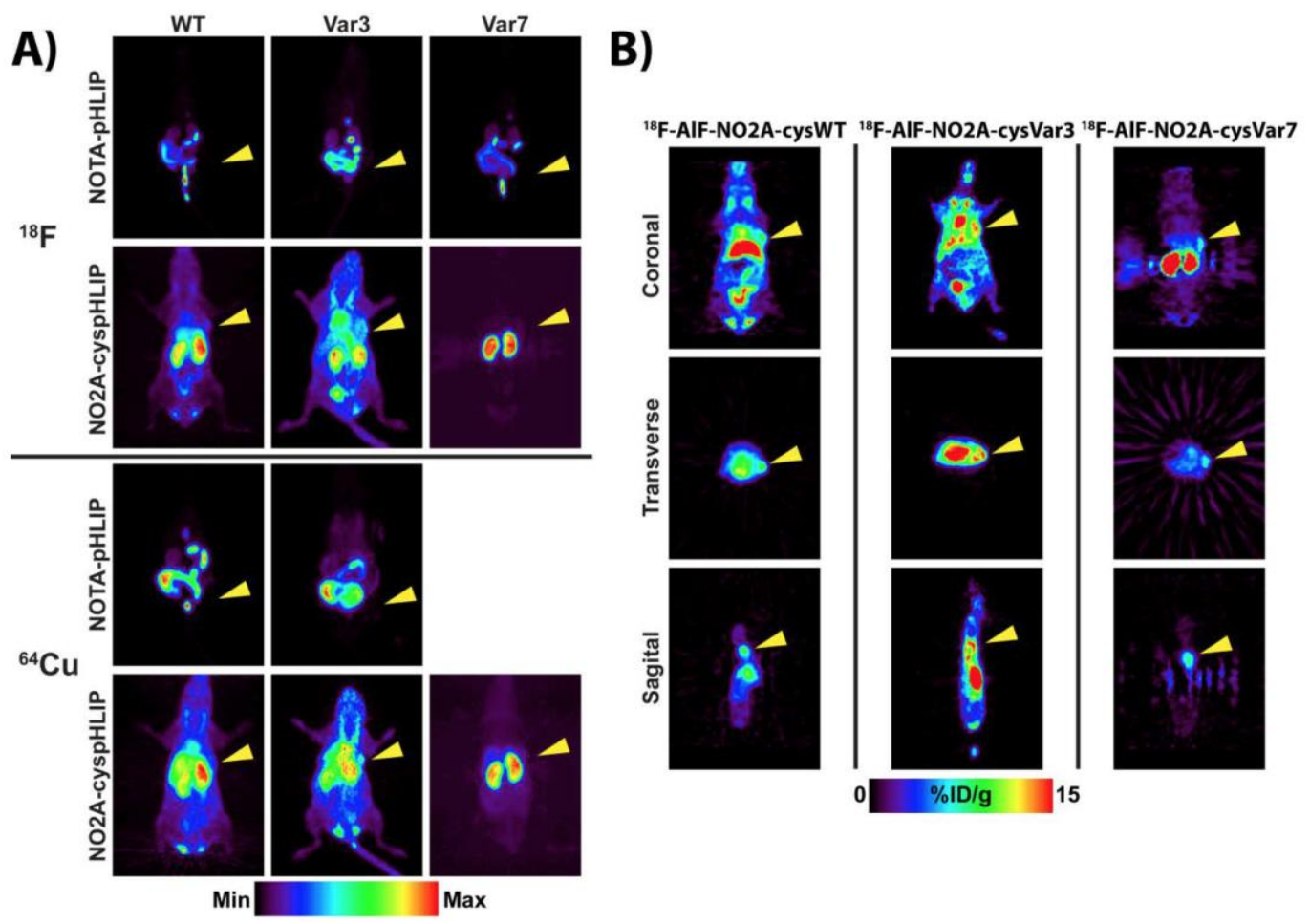

Figure S1. PET Maximum intensity projection (MIP) showing differences in the tracer distribution at $4 \mathrm{~h}$ p.i. for all 12 tracers (A) and the PET imaging slices at 15 $\% \mathrm{ID} / \mathrm{g}$ at $4 \mathrm{~h}$ p.i. for the ${ }^{18} \mathrm{~F}-\mathrm{AlF}$-cyspHLIP constructs (V) in BALB/c female mice bearing 4T1 tumor allografts. The yellow arrowheads indicate where the tumor is located in the mouse. The MIPs correspond to the same images shown in Figure 3. The slices are the same as those shown in Figure 3, just at $15 \% \mathrm{ID} / \mathrm{g}$. Only ex vivo biodistribution data was obtained for ${ }^{64} \mathrm{Cu}-\mathrm{NOTA}-\mathrm{Var} 7$. 

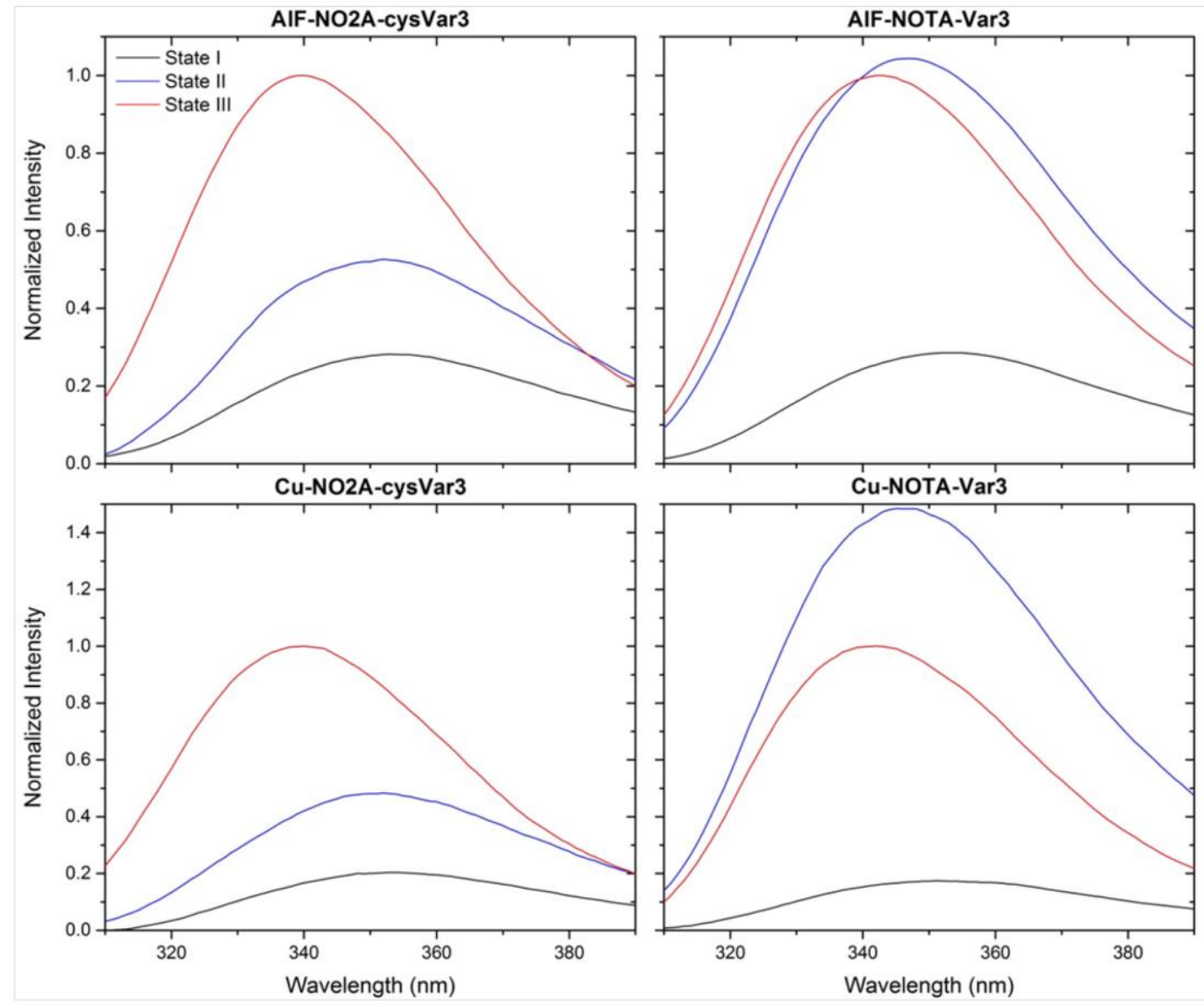

Figure S2. Three states of NO2A-cysVar3 and NOTA-Var3 constructs. The constructs were examined under varying conditions for the presence of the three states of $\mathrm{pHLIP} \circledast$ peptide: State I is the construct in solution at $\mathrm{pH} 8$ (black lines), State II is the construct in the presence of POPC liposomes at $\mathrm{pH} 8$ (blue lines), and State III is the construct folded and inserted in the lipid membrane when the $\mathrm{pH}$ is dropped from $\mathrm{pH} 8$ to $\mathrm{pH} 4$ by the addition of an aliquot of $\mathrm{HCl}$ (red lines). The concentrations of construct and lipids were $5 \mu \mathrm{M}$ and $1 \mathrm{mM}$, respectively. 


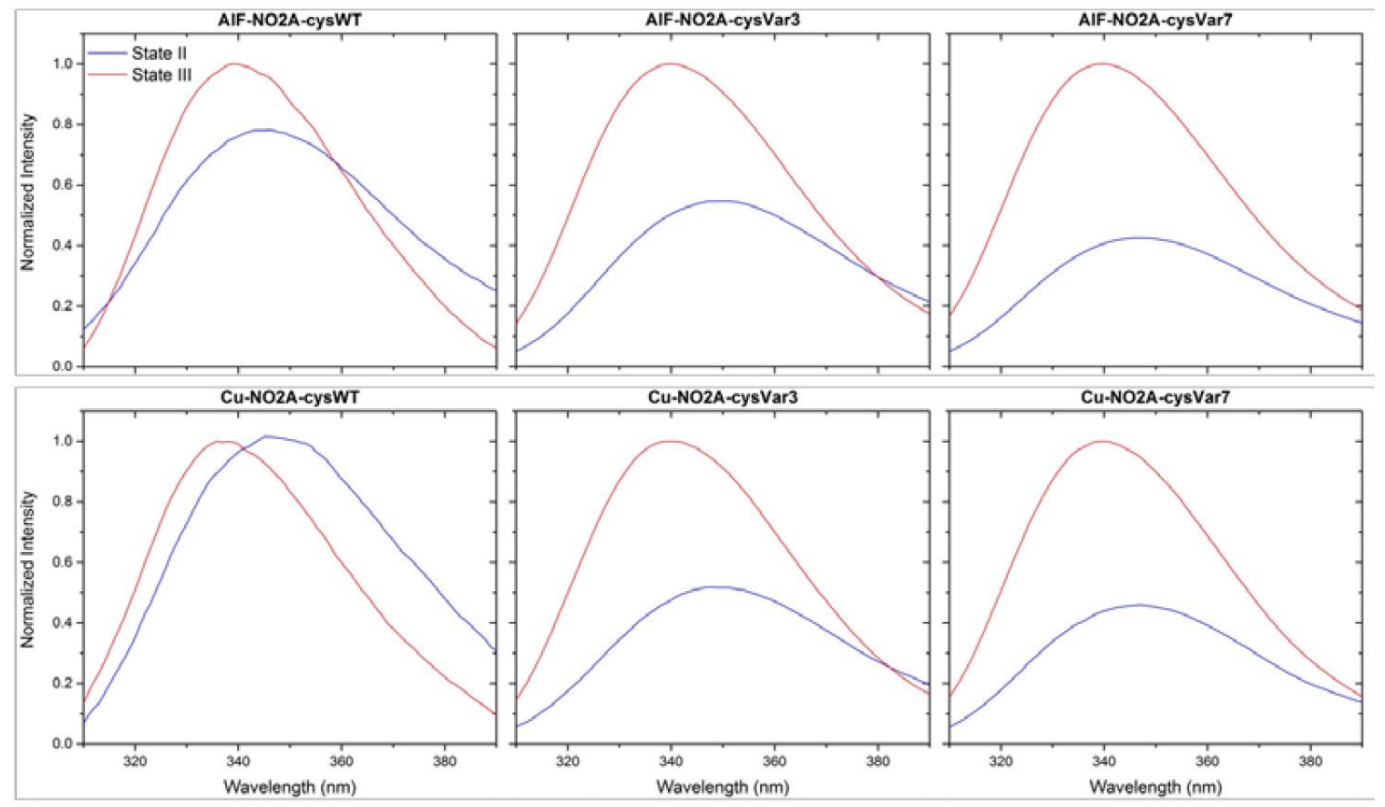

Figure S3. State II and State III of NO2A-cyspHLIP constructs. The constructs were examined in the presence of liposomes under varying $\mathrm{pH}$ levels: State II is the construct in the presence of POPC liposomes at $\mathrm{pH} 8$ (blue lines), State III is the construct folded and inserted in the lipid membrane when the $\mathrm{pH}$ is dropped from $\mathrm{pH}$ 8 to $\mathrm{pH} 4$ by the addition of an aliquot of $\mathrm{HCl}$ (red lines). The concentrations of construct and lipids were $5 \mu \mathrm{M}$ and $1 \mathrm{mM}$, respectively, with physiological levels of free magnesium and calcium ions $(0.65 \mathrm{mM}$ and $1.25 \mathrm{mM}$, respectively). 

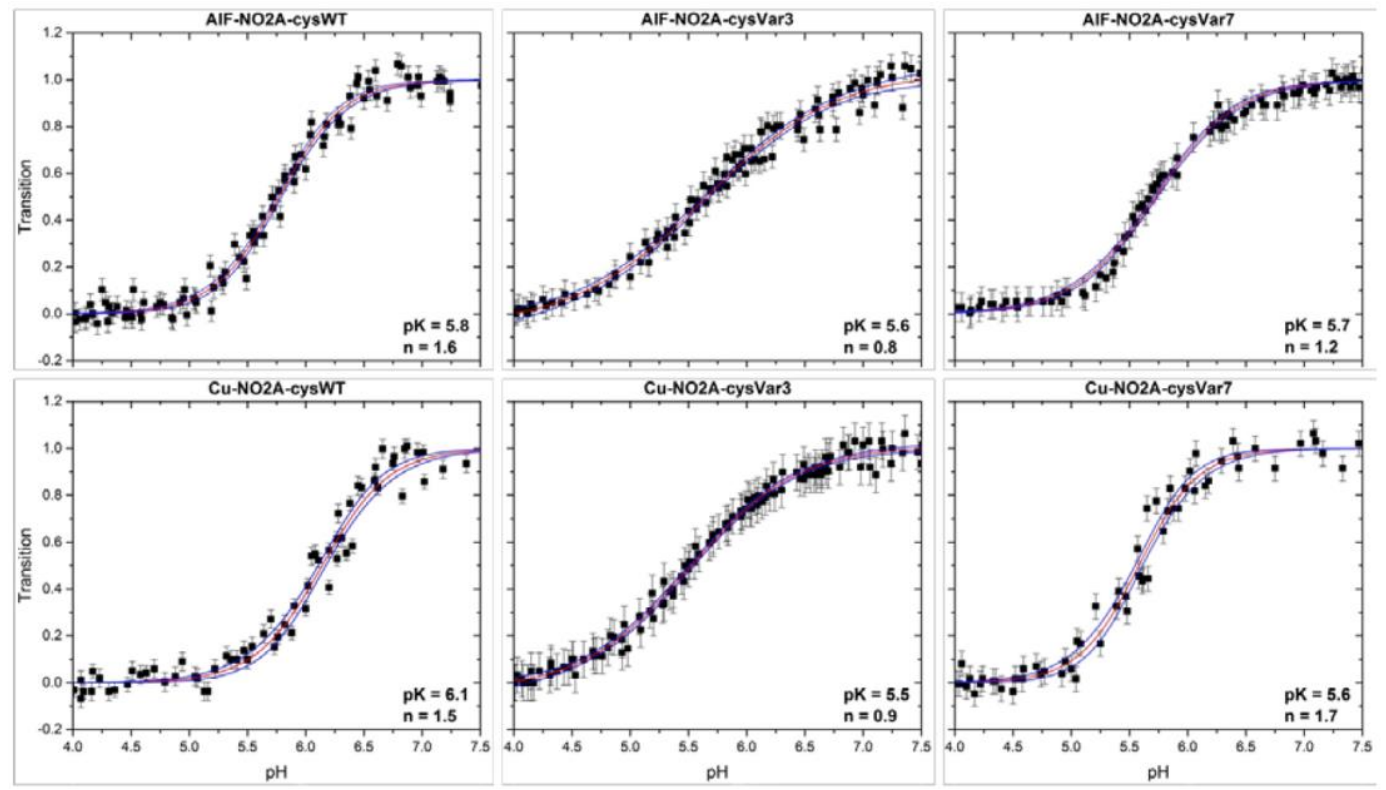

Figure S4. pH dependent bilayer insertion of constructs. Changes in intrinsic construct fluorescence are used to measure the insertion of the construct population as a function of $\mathrm{pH}$ (transition from State II, at high $\mathrm{pH}$, to State III, at low $\mathrm{pH}$ ). Amount of construct population in State II is measured on the y-axis. The red and blue lines are fitting curves and 95\% confidence intervals, respectively. These experiments were carried out with physiological levels of free magnesium and calcium ions $(0.65 \mathrm{mM}$ and $1.25 \mathrm{mM}$, respectively). 


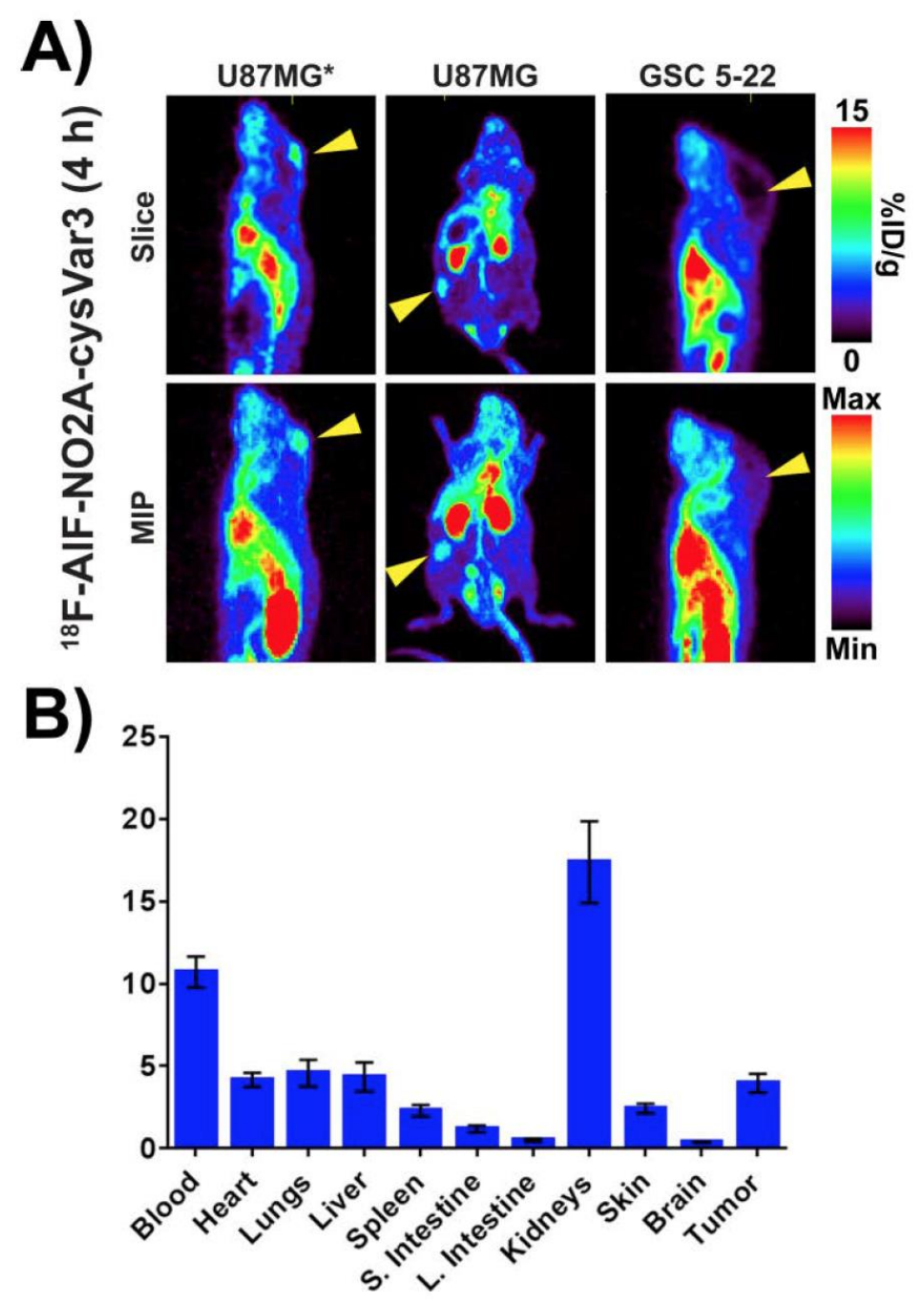

Figure S5. Initial results from brain tumor uptake study. Images of the uptake of U87MG tumors xenografted in the skull (U87MG*) or flank (U87MG) on male nude mice and GSC 5-22 tumors orthotopically xenografted behind an intact BBB in IRCSCID male mice at $4 \mathrm{~h}$ post injection (A). The U87MG* tumors did not infiltrate the brain and only the portion of the tumor that was in the skull bone and protruding from the skull showed uptake. The construct biodistribution of six male nude mice with flank U87MG xenografted tumors (B). The yellow arrowheads in (A) indicate where the tumor is located in the mouse. 


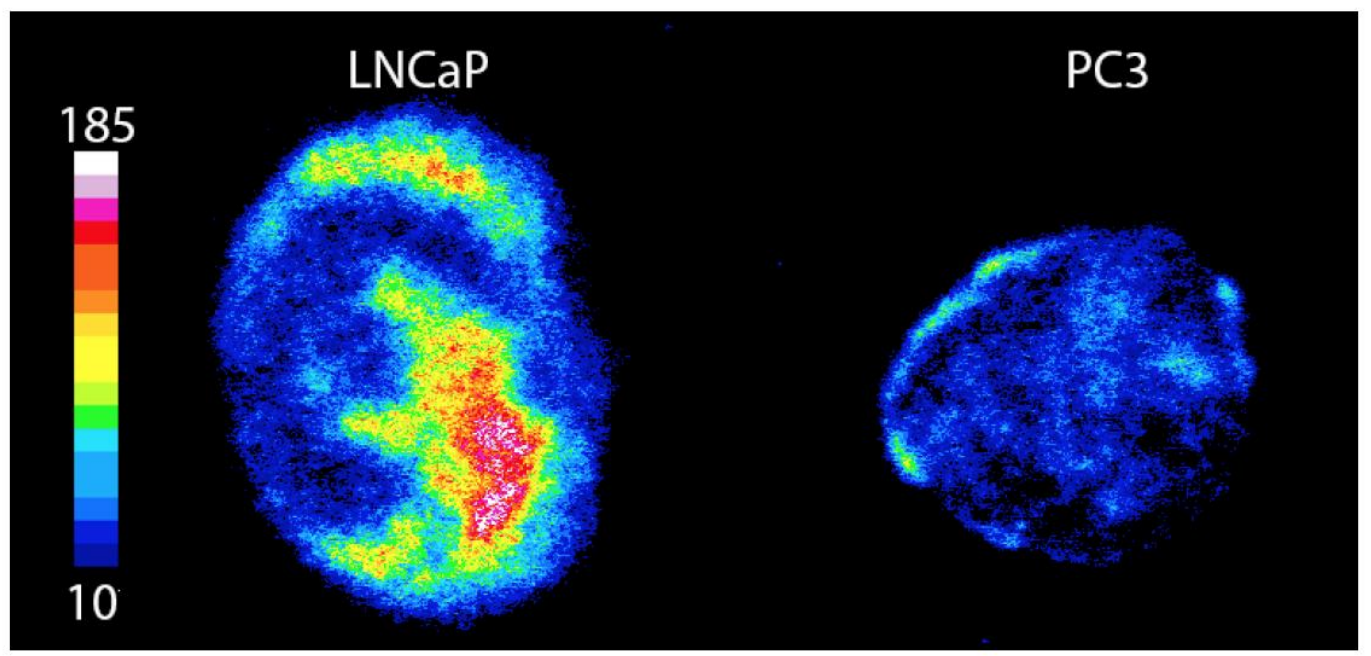

Figure S6. Relative autoradiography of tumor slices from text Figure 6. The slices show the overall difference in uptake of the tracer at $24 \mathrm{~h}$ p.i. Both of the slices were on the same autoradiography plate and, therefore, are relative to each other. 
A)

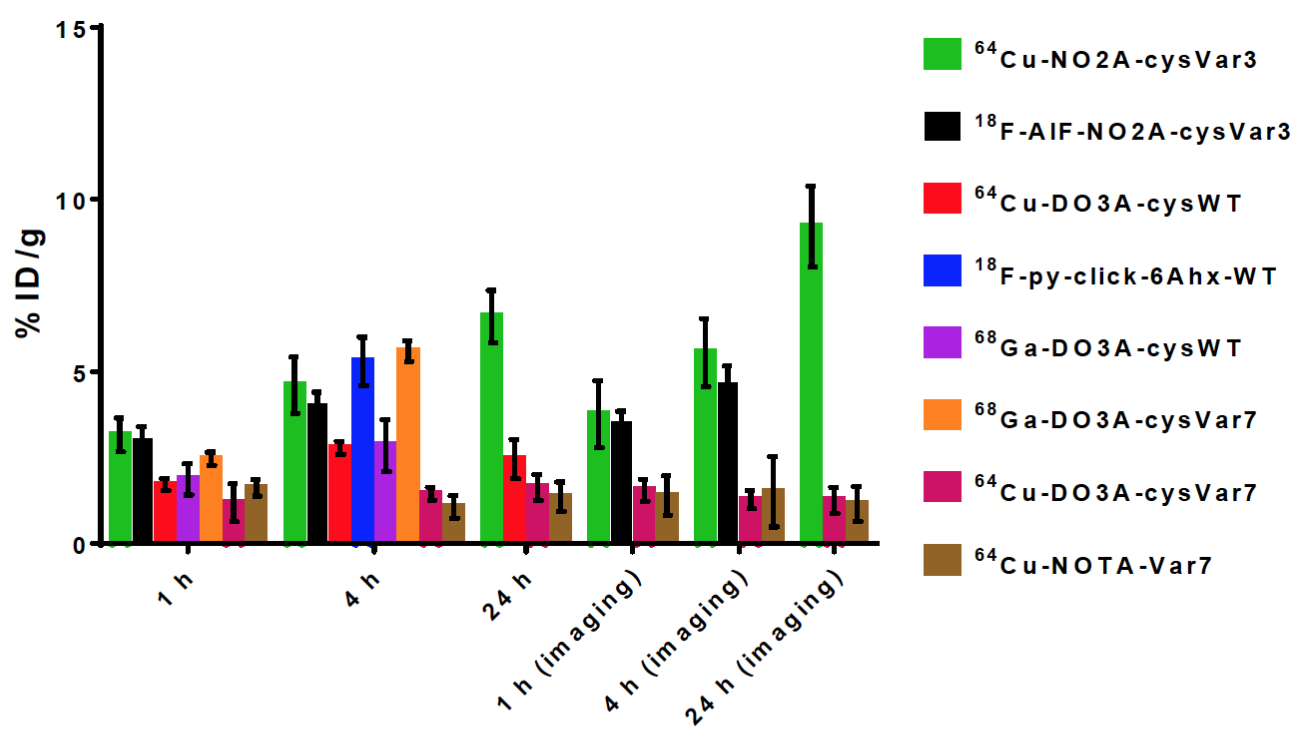

B)

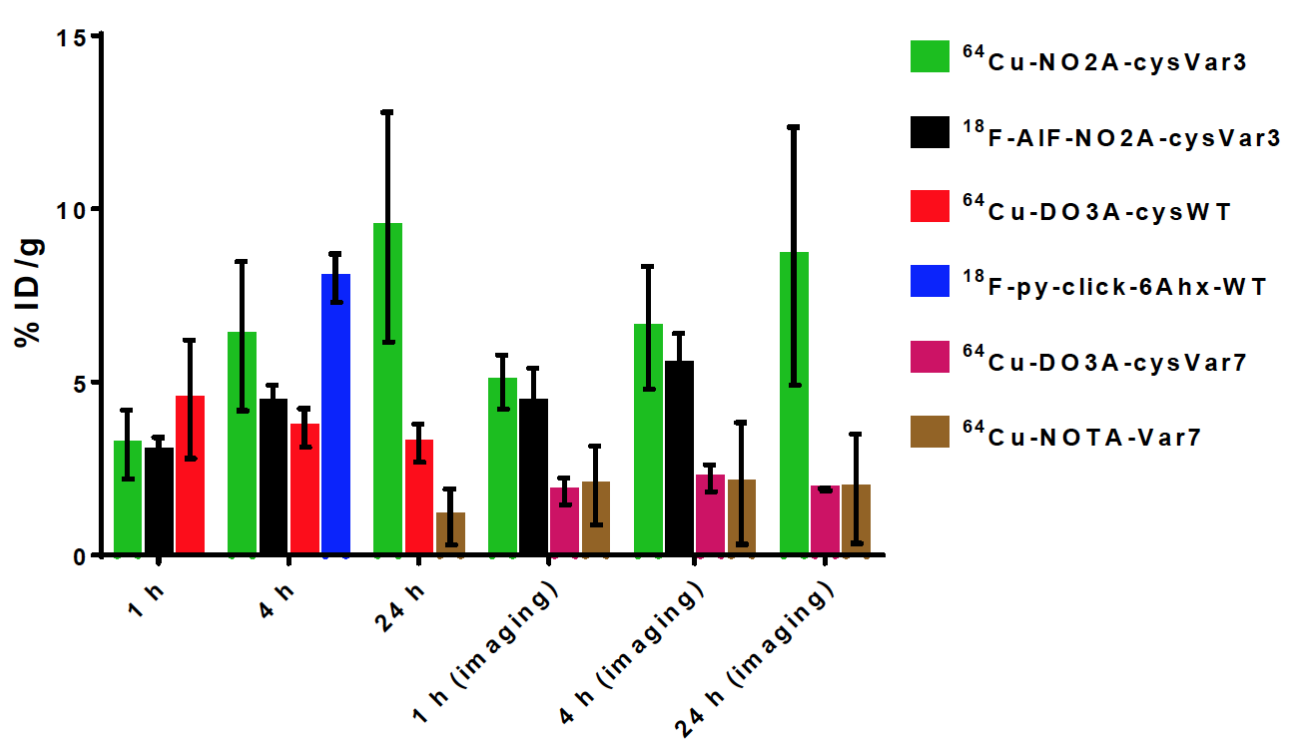

Figure S7. Comparison of tumor uptake to previously reported studies for A) PC3 and B) LNCaP tumor bearing nude male mice at 1,4 , and $24 \mathrm{~h}$ with ex vivo biodistribution and in vivo imaging values. For imaging time points, ROIs were drawn on the coronal, sagittal, and transverse slices and the middle median value was tabulated for each mouse, the average of the four mice are shown with the standard deviation. Values from previous studies were used as reported. 
A)

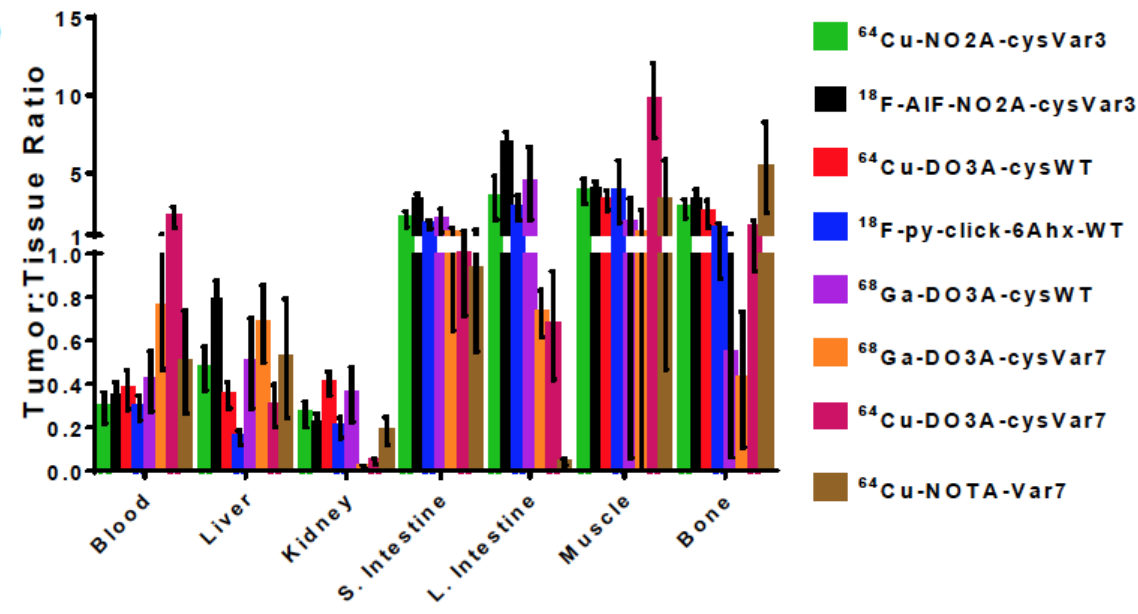

B)

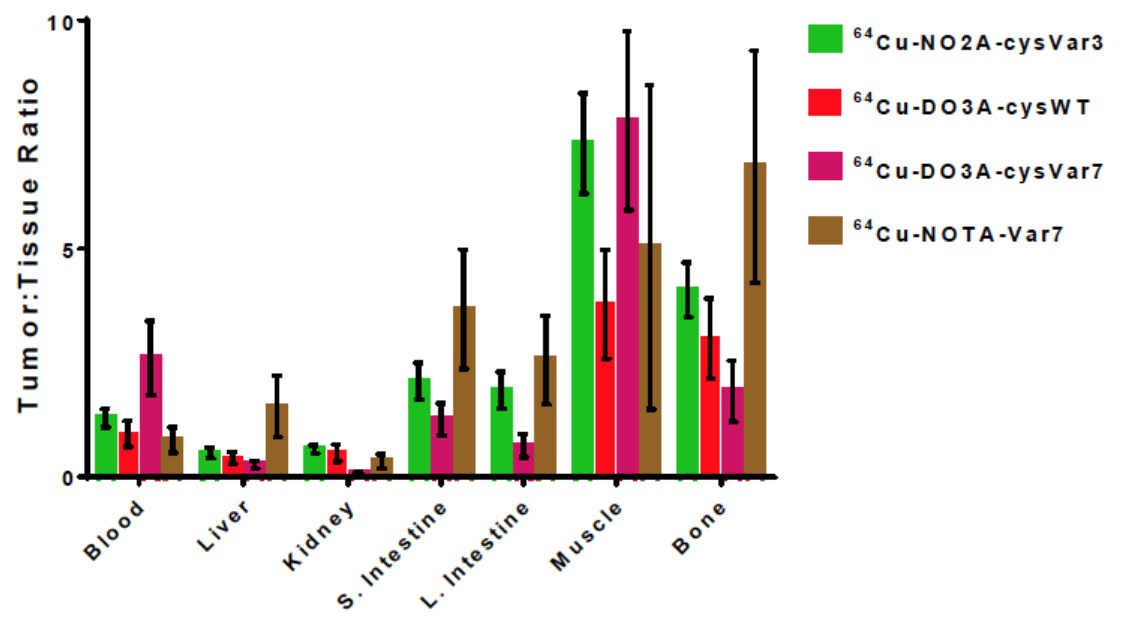

C)

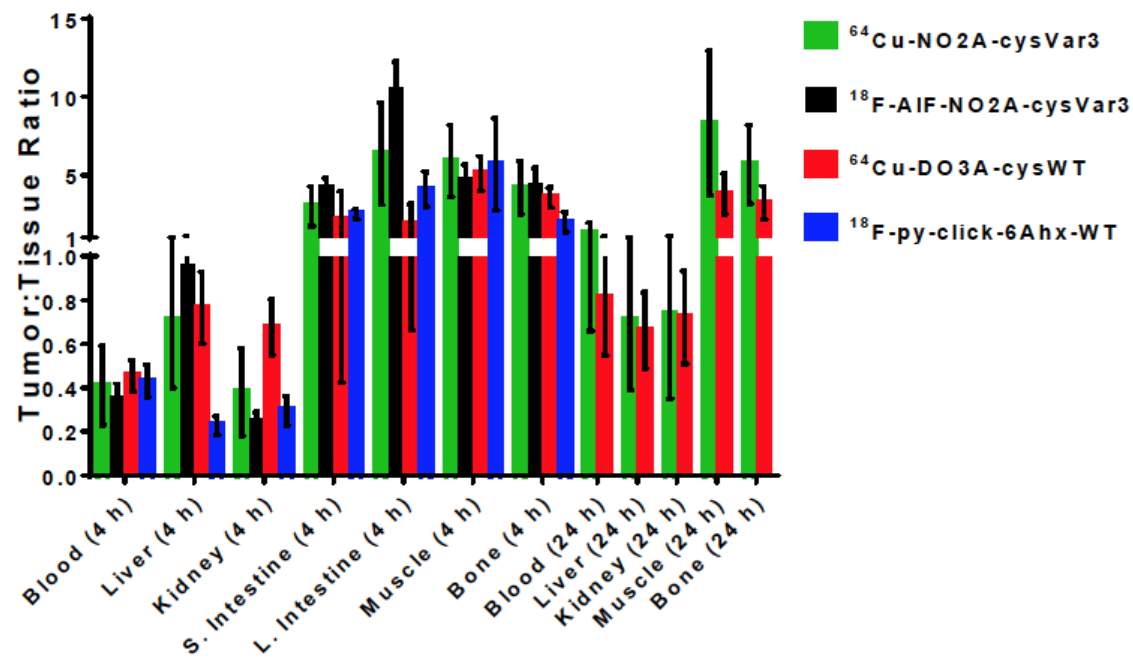

Figure S8. Comparison of tumor:tissue ratios at $4 \mathrm{~h}$ and $24 \mathrm{~h}$ to previous studies: A) PC3 at $4 \mathrm{~h}$; B) PC3 at $24 \mathrm{~h}$; C) LNCaP at 4 and $24 \mathrm{~h}$. 


\section{SUPPORTING INFORMATION TABLES}

\begin{tabular}{|c|c|c|}
\hline Imaging Agent & $\begin{array}{c}\text { Specific Activity } \\
(\mu \mathrm{Ci} / \mathrm{nmol})^{\mathrm{a}}\end{array}$ & $\begin{array}{c}\text { Specific Activity } \\
(\mu \mathrm{Ci} / \mathrm{nmol})^{b}\end{array}$ \\
\hline${ }^{64} \mathrm{Cu}-\mathrm{NOTA-Var} 7$ & 82.4 & $\mathrm{~N} / \mathrm{A}$ \\
\hline${ }^{18}$ F-AlF-NOTA-Var7 & 79.9 & $\mathrm{~N} / \mathrm{A}$ \\
\hline${ }^{64} \mathrm{Cu}-\mathrm{NOTA}$-Var3 & $132.8^{\mathrm{c}}$ & $\mathrm{N} / \mathrm{A}$ \\
\hline${ }^{18}$ F-AIF-NOTA-Var3 & 101.3 & $\mathrm{~N} / \mathrm{A}$ \\
\hline${ }^{64} \mathrm{Cu}-\mathrm{NOTA-WT}$ & 116.4 & $\mathrm{~N} / \mathrm{A}$ \\
\hline${ }^{18}$ F-AIF-NOTA-WT & 135.9 & $\mathrm{~N} / \mathrm{A}$ \\
\hline${ }^{64} \mathrm{Cu}-\mathrm{NO} 2 \mathrm{~A}-\mathrm{cys} V$ ar7 & 113.7 & 219.6 \\
\hline${ }^{18}$ F-AIF-NO2A-cysVar7 & 55.9 & 178.1 \\
\hline${ }^{64} \mathrm{Cu}-\mathrm{NO2A-cysVar3}$ & $45.5-523.3$ & $47.5-340.2$ \\
\hline${ }^{18}$ F-AIF-NO2A-cysVar3 & $31.2-84.2$ & $38.9-351.4$ \\
\hline${ }^{64} \mathrm{Cu}-\mathrm{NO2A}$-cysWT & 215.6 & 99.1 \\
\hline${ }^{18}$ F-AIF-NO2A-cysWT & 19.6 & $\mathrm{~N} / \mathrm{A}$ \\
\hline
\end{tabular}

Table S1. General specific activities of tracers produced for this study. ${ }^{\text {a Approximated }}$ from the amount of total peptide added. ${ }^{\mathrm{b}}$ Calculated from the UV/vis standard concentration curve. ${ }^{\mathrm{C}}$ In a separate experiment, a specific activity of $1656 \mu \mathrm{Ci} / \mathrm{nmol}$ was attained, but the lower specific activity material was used in the studies in order to be comparable throughout. 
DATA FOR EACH TRACER FROM THIS STUDY IN FEMALE, BALB/C MICE WITH ORTHOTOPIC 4T1 BREAST CANCER ALLOGRAFTS ${ }^{64} \mathrm{Cu}$-NOTA-WT

\begin{tabular}{lccccccccccccccc}
\hline Tissue & \multicolumn{1}{c}{$\mathbf{1} \mathbf{h}(\mathbf{n}=\mathbf{4})$} & \multicolumn{2}{c}{$\mathbf{2} \mathbf{h}(\mathbf{n}=\mathbf{4})$} & \multicolumn{2}{c}{$\mathbf{4} \mathbf{h}(\mathbf{n}=\mathbf{4})$} & \multicolumn{1}{c}{$\mathbf{1 2} \mathbf{h}(\mathbf{n}=\mathbf{4})$} & $\mathbf{2 4} \mathbf{h}(\mathbf{n}=\mathbf{3})$ \\
\hline Blood & 0.585 & \pm & 0.291 & 0.413 & \pm & 0.265 & 0.172 & \pm & 0.047 & 0.533 & \pm & 0.118 & 0.288 & \pm & 0.119 \\
Heart & 0.057 & \pm & 0.006 & 0.046 & \pm & 0.006 & 0.048 & \pm & 0.009 & 0.059 & \pm & 0.009 & 0.040 & \pm & 0.005 \\
Lungs & 0.359 & \pm & 0.043 & 0.364 & \pm & 0.049 & 0.314 & \pm & 0.041 & 0.340 & \pm & 0.074 & 0.236 & \pm & 0.006 \\
Liver & 1.185 & \pm & 0.298 & 0.991 & \pm & 0.219 & 0.822 & \pm & 0.150 & 0.858 & \pm & 0.166 & 0.645 & \pm & 0.024 \\
Spleen & 0.049 & \pm & 0.009 & 0.039 & \pm & 0.005 & 0.047 & \pm & 0.007 & 0.052 & \pm & 0.008 & 0.038 & \pm & 0.004 \\
Pancreas & 0.041 & \pm & 0.015 & 0.032 & \pm & 0.005 & 0.044 & \pm & 0.019 & 0.037 & \pm & 0.006 & 0.031 & \pm & 0.005 \\
Stomach & 0.282 & \pm & 0.244 & 0.104 & \pm & 0.028 & 0.121 & \pm & 0.043 & 0.109 & \pm & 0.019 & 0.074 & \pm & 0.003 \\
S. intestine & 12.043 & \pm & 2.341 & 1.684 & \pm & 0.580 & 0.444 & \pm & 0.050 & 0.376 & \pm & 0.021 & 0.280 & \pm & 0.010 \\
L. intestine & 2.486 & \pm & 2.911 & 14.250 & \pm & 1.086 & 8.510 & \pm & 3.356 & 0.540 & \pm & 0.138 & 0.291 & \pm & 0.054 \\
Kidneys & 2.213 & \pm & 0.110 & 1.849 & \pm & \pm .136 & 1.739 & \pm & 0.183 & 1.434 & \pm & 0.050 & 1.107 & \pm & 0.094 \\
Muscle & 0.020 & \pm & 0.005 & 0.017 & \pm & \pm .004 & 0.017 & \pm & 0.004 & 0.018 & \pm & 0.004 & 0.012 & \pm & 0.004 \\
Bone & 0.012 & \pm & 0.008 & 0.012 & \pm & 0.004 & 0.014 & \pm & 0.004 & 0.026 & \pm & 0.015 & 0.012 & \pm & 0.003 \\
Skin & 0.070 & \pm & 0.023 & 0.080 & \pm & 0.022 & 0.054 & \pm & 0.007 & 0.095 & \pm & 0.013 & 0.074 & \pm & 0.012 \\
Brain & 0.033 & \pm & 0.008 & 0.026 & \pm & 0.005 & 0.022 & \pm & 0.003 & 0.023 & \pm & 0.001 & 0.017 & \pm & 0.001 \\
Tumor & 0.101 & \pm & 0.019 & 0.101 & \pm & 0.012 & 0.136 & \pm & 0.027 & 0.192 & \pm & 0.071 & 0.198 & \pm & 0.025 \\
\hline
\end{tabular}

Table S1-1. Tissue uptake (mean \%ID \pm SD) of ${ }^{64} \mathrm{Cu}-\mathrm{NOTA}-\mathrm{WT}$ administered via the lateral tail vein in female, BALB/c mice with orthotopic $4 \mathrm{~T} 1$ breast cancer allografts implanted into the mammary fat pad. 


\begin{tabular}{|c|c|c|c|c|c|c|c|c|c|c|c|c|c|c|c|}
\hline \multirow{2}{*}{$\frac{\text { Tissue }}{\text { Blood }}$} & \multicolumn{3}{|c|}{$1 \mathrm{~h}(\mathrm{n}=4)$} & \multicolumn{3}{|c|}{$2 h(n=4)$} & \multicolumn{3}{|c|}{$4 h(n=4)$} & \multicolumn{3}{|c|}{$12 h(n=4)$} & \multicolumn{3}{|c|}{24 h $(n=3)$} \\
\hline & 1.529 & \pm & 0.164 & 1.223 & \pm & 0.255 & 1.192 & \pm & 0.263 & 1.241 & \pm & 0.225 & 0.862 & \pm & 0.097 \\
\hline Heart & 0.589 & \pm & 0.061 & 0.476 & \pm & 0.051 & 0.495 & \pm & 0.042 & 0.519 & \pm & 0.099 & 0.364 & \pm & 0.036 \\
\hline Lungs & 1.773 & \pm & 0.161 & 1.470 & \pm & 0.090 & 1.414 & \pm & 0.083 & 1.252 & \pm & 0.231 & 0.886 & \pm & 0.106 \\
\hline Liver & 1.496 & \pm & 0.379 & 1.161 & \pm & 0.231 & 0.904 & \pm & 0.097 & 0.816 & \pm & 0.180 & 0.637 & \pm & 0.063 \\
\hline Spleen & 0.348 & \pm & 0.045 & 0.308 & \pm & 0.021 & 0.318 & \pm & 0.023 & 0.342 & \pm & 0.050 & 0.272 & \pm & 0.017 \\
\hline Pancreas & 0.267 & \pm & 0.082 & 0.223 & \pm & 0.027 & 0.261 & \pm & 0.066 & 0.266 & \pm & 0.091 & 0.188 & \pm & 0.031 \\
\hline Stomach & 0.551 & \pm & 0.264 & 0.352 & \pm & 0.085 & 0.282 & \pm & 0.080 & 0.302 & \pm & 0.055 & 0.170 & \pm & 0.023 \\
\hline S. intestine & 11.075 & \pm & 3.605 & 1.410 & \pm & 0.447 & 0.391 & \pm & 0.082 & 0.301 & \pm & 0.026 & 0.198 & \pm & 0.023 \\
\hline L. intestine & 4.140 & \pm & 4.791 & 20.733 & \pm & 1.782 & 9.956 & \pm & 3.415 & 0.648 & \pm & 0.192 & 0.291 & \pm & 0.063 \\
\hline Kidneys & 8.326 & \pm & 1.158 & 7.156 & \pm & 0.725 & 6.232 & \pm & 0.613 & 4.974 & \pm & 0.766 & 3.527 & \pm & 0.943 \\
\hline Muscle & 0.191 & \pm & 0.026 & 0.164 & \pm & 0.026 & 0.166 & \pm & 0.008 & 0.160 & \pm & 0.019 & 0.124 & \pm & 0.028 \\
\hline Bone & 0.251 & \pm & 0.063 & 0.234 & \pm & 0.089 & 0.274 & \pm & 0.051 & 0.348 & \pm & 0.063 & 0.269 & \pm & 0.042 \\
\hline Skin & 0.700 & \pm & 0.072 & 0.679 & \pm & 0.102 & 0.715 & \pm & 0.109 & 0.766 & \pm & 0.116 & 0.512 & \pm & 0.062 \\
\hline Brain & 0.092 & \pm & 0.012 & 0.069 & \pm & 0.008 & 0.054 & \pm & 0.002 & 0.057 & \pm & 0.007 & 0.046 & \pm & 0.003 \\
\hline Tumor & 0.577 & \pm & 0.074 & 0.574 & \pm & 0.108 & 0.679 & \pm & 0.025 & 0.904 & \pm & 0.144 & 0.845 & \pm & 0.127 \\
\hline \multicolumn{16}{|c|}{ Tumor-to-tissue ratios (rel. u.) } \\
\hline Tumor/blood & 0.377 & \pm & 0.063 & 0.470 & \pm & 0.132 & 0.569 & \pm & 0.127 & 0.729 & \pm & 0.176 & 0.980 & \pm & 0.184 \\
\hline Tumor/liver & 0.386 & \pm & 0.110 & 0.494 & \pm & 0.135 & 0.751 & \pm & 0.085 & 1.108 & \pm & 0.301 & 1.327 & \pm & 0.239 \\
\hline Tumor/S. I. & 0.052 & \pm & 0.018 & 0.407 & \pm & 0.150 & 1.737 & \pm & 0.370 & 3.008 & \pm & 0.543 & 4.264 & \pm & 0.804 \\
\hline Tumor/L. I. & 0.139 & \pm & 0.162 & 0.028 & \pm & 0.006 & 0.068 & \pm & 0.024 & 1.395 & \pm & 0.469 & 2.904 & \pm & 0.767 \\
\hline Tumor/kidney & 0.069 & \pm & 0.013 & 0.080 & \pm & 0.017 & 0.109 & \pm & 0.011 & 0.182 & \pm & 0.040 & 0.240 & \pm & 0.073 \\
\hline Tumor/muscle & 3.029 & \pm & 0.565 & 3.509 & \pm & 0.869 & 4.099 & \pm & 0.255 & 5.665 & \pm & 1.130 & 6.794 & \pm & 1.832 \\
\hline Tumor/bone & 2.301 & \pm & 0.651 & 2.457 & \pm & 1.048 & 2.473 & \pm & 0.471 & 2.601 & \pm & 0.626 & 3.146 & \pm & 0.682 \\
\hline
\end{tabular}

Table S1-2. Tissue uptake (mean $\% \mathrm{ID} / \mathrm{g} \pm \mathrm{SD}$ ) of ${ }^{64} \mathrm{Cu}-\mathrm{NOTA}-\mathrm{WT}$ administered via the lateral tail vein in female, BALB/c mice with orthotopic 4T1 breast cancer allografts implanted into the mammary fat pad. 


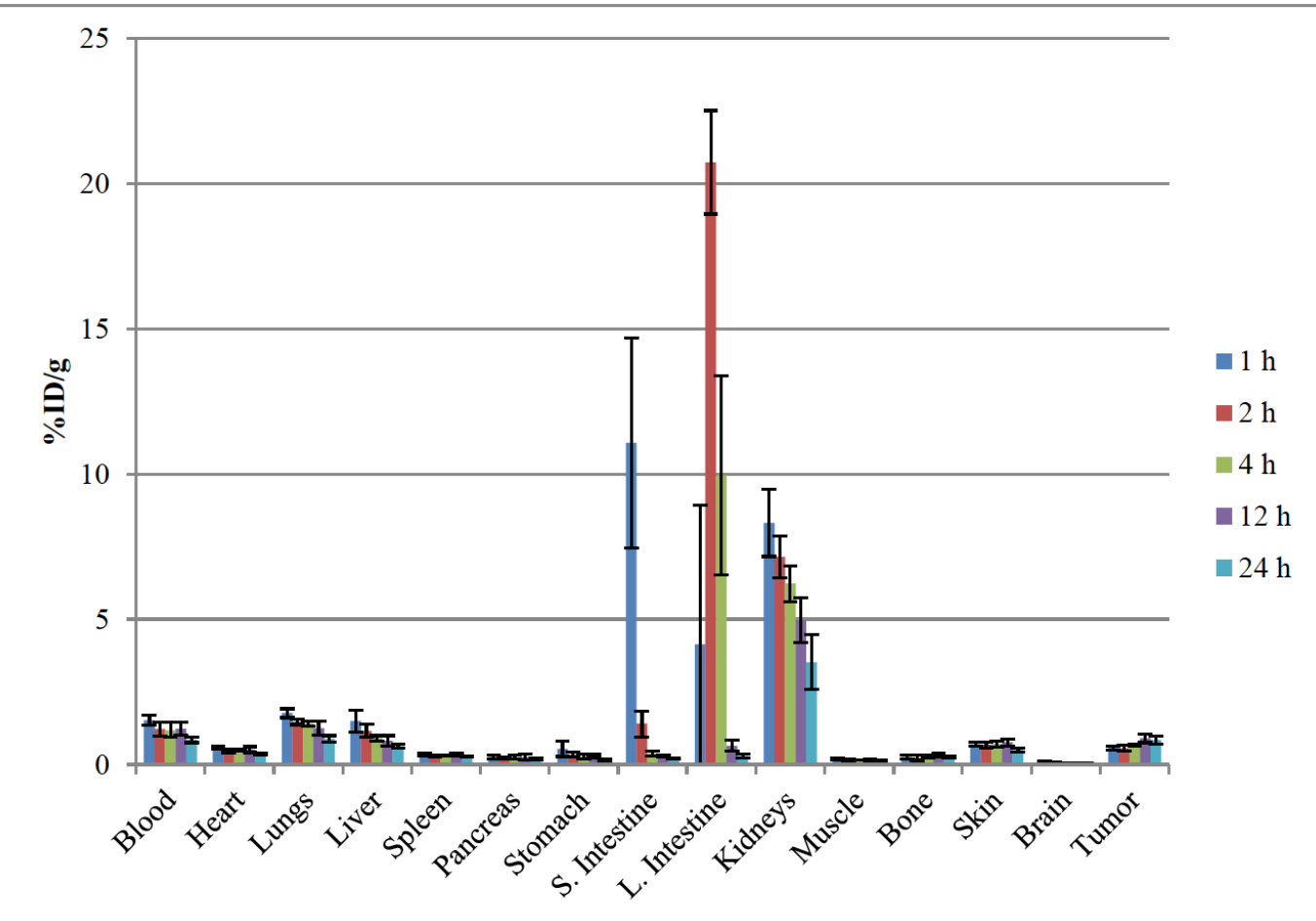

Fig. S1-1. Tissue uptake (mean $\% \mathrm{ID} / \mathrm{g} \pm \mathrm{SD})$ of ${ }^{64} \mathrm{Cu}-\mathrm{NOTA}-\mathrm{WT}(\mathrm{n}=4(3$ at $24 \mathrm{~h})$ ) administered via the lateral tail vein in female, BALB/c mice with orthotopic 4T1 breast cancer allografts implanted into the mammary fat pad full graph from text. 


\section{BIOSCAN}

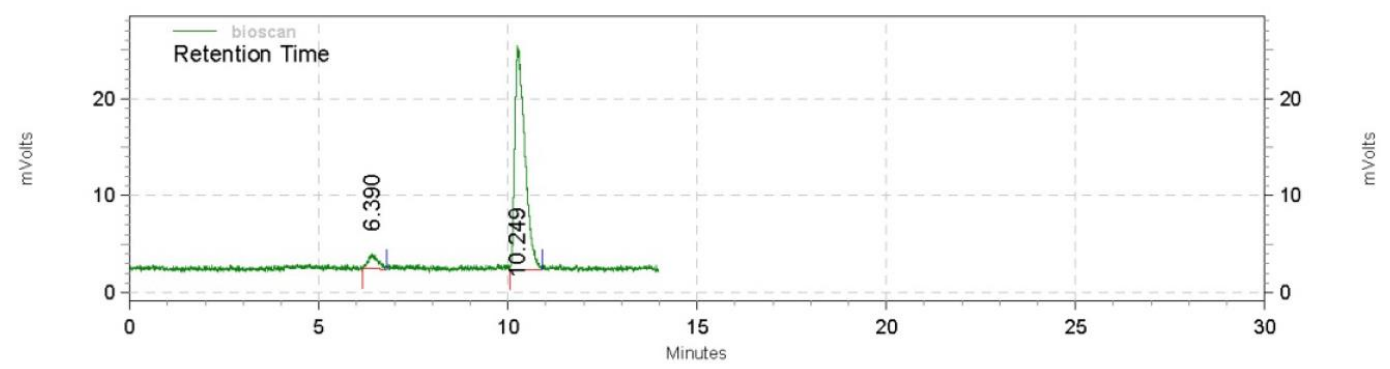

\begin{tabular}{rrrrrr|}
$\begin{array}{c}\text { bioscan Results } \\
\text { Retention Time }\end{array}$ & Area & Area \% & Height & Height \% \\
\hline 6.390 & 25927 & 5.77 & 1475 & 6.01 \\
10.249 & 423382 & 94.23 & 23073 & 93.99 \\
\hline Totals & 449309 & 100.00 & 24548 & 100.00 \\
\hline
\end{tabular}

Fig. S1-2. HPLC Chromatogram of the Reconstituted ${ }^{64} \mathrm{Cu}-\mathrm{NOTA}-W T$ Injectate 
${ }^{64} \mathrm{Cu}-\mathrm{NOTA}-\mathrm{Var} 3$

\begin{tabular}{lccccccccccccccc}
\hline Tissue & \multicolumn{2}{c}{$\mathbf{1} \mathbf{h}(\mathbf{n}=\mathbf{5})$} & \multicolumn{2}{c}{$\mathbf{2} \mathbf{h}(\mathbf{n}=\mathbf{5})$} & \multicolumn{2}{c}{$\mathbf{4} \mathbf{h}(\mathbf{n}=\mathbf{5})$} & \multicolumn{1}{c}{$\mathbf{1 2} \mathbf{h}(\mathbf{n}=\mathbf{5})$} & \multicolumn{2}{c}{$\mathbf{2 4} \mathbf{h}(\mathbf{n}=\mathbf{4})$} \\
\hline Blood & 0.288 & \pm & 0.059 & 0.271 & \pm & 0.185 & 0.225 & \pm & 0.122 & 0.232 & \pm & 0.048 & 0.479 & \pm & 0.214 \\
Heart & 0.057 & \pm & 0.014 & 0.052 & \pm & 0.007 & 0.047 & \pm & 0.009 & 0.039 & \pm & 0.004 & 0.041 & \pm & 0.012 \\
Lungs & 0.420 & \pm & 0.047 & 0.458 & \pm & 0.058 & 0.388 & \pm & 0.073 & 0.303 & \pm & 0.067 & 0.276 & \pm & 0.044 \\
Liver & 1.093 & \pm & 0.224 & 0.892 & \pm & 0.188 & 0.808 & \pm & 0.081 & 0.713 & \pm & 0.042 & 0.647 & \pm & 0.140 \\
Spleen & 0.047 & \pm & 0.008 & 0.048 & \pm & 0.005 & 0.045 & \pm & 0.002 & 0.050 & \pm & 0.010 & 0.045 & \pm & 0.006 \\
Pancreas & 0.052 & \pm & 0.015 & 0.044 & \pm & 0.021 & 0.045 & \pm & 0.011 & 0.037 & \pm & 0.009 & 0.041 & \pm & 0.023 \\
Stomach & 0.092 & \pm & 0.018 & 0.119 & \pm & 0.041 & 0.111 & \pm & 0.013 & 0.115 & \pm & 0.025 & 0.096 & \pm & 0.046 \\
S. intestine & 17.226 & \pm & \pm .329 & 1.364 & \pm & 0.327 & 0.394 & \pm & 0.075 & 0.335 & \pm & 0.029 & 0.322 & \pm & 0.063 \\
L. intestine & 1.859 & \pm & \pm .941 & 18.364 & \pm & 3.419 & 5.350 & \pm & 3.021 & 0.514 & \pm & 0.128 & 0.342 & \pm & 0.026 \\
Kidneys & 2.591 & \pm & 0.170 & 2.141 & \pm & 0.203 & 1.955 & \pm & 0.280 & 1.454 & \pm & 0.097 & 1.266 & \pm & 0.178 \\
Muscle & 0.017 & \pm & 0.007 & 0.020 & \pm & 0.004 & 0.018 & \pm & 0.001 & 0.014 & \pm & 0.003 & 0.016 & \pm & 0.006 \\
Bone & 0.016 & \pm & 0.015 & 0.011 & \pm & 0.006 & 0.022 & \pm & 0.009 & 0.020 & \pm & 0.008 & 0.008 & \pm & 0.006 \\
Skin & 0.055 & \pm & 0.016 & 0.062 & \pm & 0.009 & 0.060 & \pm & 0.013 & 0.056 & \pm & 0.018 & 0.062 & \pm & 0.016 \\
Brain & 0.033 & \pm & 0.004 & 0.030 & \pm & 0.007 & 0.022 & \pm & 0.004 & 0.018 & \pm & 0.002 & 0.017 & \pm & 0.002 \\
Tumor & 0.110 & \pm & 0.029 & 0.124 & \pm & 0.024 & 0.145 & \pm & 0.048 & 0.165 & \pm & 0.029 & 0.196 & \pm & 0.026 \\
\hline
\end{tabular}

Table S2-1. Tissue uptake (mean \% ID \pm SD) of ${ }^{64} \mathrm{Cu}$-NOTA-var3 administered via

the lateral tail vein in female, BALB/c mice with orthotopic $4 \mathrm{~T} 1$ breast cancer allografts implanted into the mammary fat pad. 


\begin{tabular}{|c|c|c|c|c|c|c|c|c|c|c|c|c|c|c|c|}
\hline \multirow{2}{*}{$\begin{array}{l}\text { Tissue } \\
\text { Blood }\end{array}$} & \multicolumn{3}{|c|}{$1 \mathrm{~h}(\mathrm{n}=5)$} & \multicolumn{3}{|c|}{$2 \mathrm{~h}(\mathrm{n}=5)$} & \multicolumn{3}{|c|}{$4 h(n=5)$} & \multicolumn{3}{|c|}{$12 h(n=5)$} & \multicolumn{3}{|c|}{$24 h(n=4)$} \\
\hline & 1.215 & \pm & 0.223 & 1.226 & \pm & 0.361 & 1.371 & \pm & 0.244 & 1.113 & \pm & 0.136 & 1.073 & \pm & 0.223 \\
\hline Heart & 0.509 & \pm & 0.103 & 0.490 & \pm & 0.067 & 0.489 & \pm & 0.047 & 0.401 & \pm & 0.052 & 0.404 & \pm & 0.104 \\
\hline Lungs & 1.516 & \pm & 0.212 & 1.499 & \pm & 0.231 & 1.420 & \pm & 0.117 & 1.048 & \pm & 0.180 & 1.079 & \pm & 0.179 \\
\hline Liver & 1.273 & \pm & 0.109 & 1.073 & \pm & 0.197 & 1.034 & \pm & 0.150 & 0.631 & \pm & 0.067 & 0.719 & \pm & 0.125 \\
\hline Spleen & 0.305 & \pm & 0.047 & 0.333 & \pm & 0.029 & 0.340 & \pm & 0.032 & 0.289 & \pm & 0.019 & 0.308 & \pm & 0.055 \\
\hline Pancreas & 0.291 & \pm & 0.070 & 0.261 & \pm & 0.057 & 0.279 & \pm & 0.041 & 0.219 & \pm & 0.029 & 0.203 & \pm & 0.045 \\
\hline Stomach & 0.267 & \pm & 0.091 & 0.240 & \pm & 0.054 & 0.194 & \pm & 0.050 & 0.227 & \pm & 0.074 & 0.250 & \pm & 0.038 \\
\hline S. intestine & 13.092 & \pm & 2.311 & 1.034 & \pm & 0.352 & 0.320 & \pm & 0.042 & 0.263 & \pm & 0.024 & 0.259 & \pm & 0.043 \\
\hline L. intestine & 1.988 & \pm & 1.539 & 25.768 & \pm & 5.740 & 7.449 & \pm & 2.726 & 0.507 & \pm & 0.065 & 0.395 & \pm & 0.058 \\
\hline Kidneys & 9.335 & \pm & 0.826 & 7.715 & \pm & 0.750 & 7.165 & \pm & 1.126 & 4.735 & \pm & 0.413 & 4.397 & \pm & 0.874 \\
\hline Muscle & 0.187 & \pm & 0.019 & 0.198 & \pm & 0.052 & 0.171 & \pm & 0.017 & 0.143 & \pm & 0.031 & 0.152 & \pm & 0.051 \\
\hline Bone & 0.216 & \pm & 0.113 & 0.269 & \pm & 0.078 & 0.339 & \pm & 0.018 & 0.320 & \pm & 0.070 & 0.220 & \pm & 0.136 \\
\hline Skin & 0.728 & \pm & 0.205 & 0.720 & \pm & 0.099 & 0.775 & \pm & 0.102 & 0.699 & \pm & 0.192 & 0.672 & \pm & 0.187 \\
\hline Brain & 0.087 & \pm & 0.013 & 0.076 & \pm & 0.018 & 0.063 & \pm & 0.011 & 0.046 & \pm & 0.004 & 0.042 & \pm & 0.010 \\
\hline Tumor & 0.515 & \pm & 0.083 & 0.663 & \pm & 0.167 & 0.649 & \pm & 0.091 & 0.755 & \pm & 0.056 & 0.926 & \pm & 0.175 \\
\hline \multicolumn{16}{|c|}{ Tumor-to-tissue ratios (rel. u.) } \\
\hline Tumor/Blood & 0.42 & \pm & 0.10 & 0.54 & \pm & 0.21 & 0.47 & \pm & 0.11 & 0.68 & \pm & 0.10 & 0.86 & \pm & 0.24 \\
\hline Tumor/Liver & 0.40 & \pm & 0.07 & 0.62 & \pm & 0.19 & 0.63 & \pm & 0.13 & 1.20 & \pm & 0.15 & 1.29 & \pm & 0.33 \\
\hline Tumor/S. I. & 0.04 & \pm & 0.01 & 0.64 & \pm & 0.27 & 2.03 & \pm & 0.39 & 2.87 & \pm & 0.34 & 3.57 & \pm & 0.90 \\
\hline Tumor/L. I. & 0.26 & \pm & 0.20 & 0.03 & \pm & 0.01 & 0.09 & \pm & 0.03 & 1.49 & \pm & 0.22 & 2.35 & \pm & 0.56 \\
\hline Tumor/Kidney & 0.06 & \pm & 0.01 & 0.09 & \pm & 0.02 & 0.09 & \pm & 0.02 & 0.16 & \pm & 0.02 & 0.21 & \pm & 0.06 \\
\hline Tumor/Muscle & 2.75 & \pm & 0.52 & 3.34 & \pm & 1.22 & 3.80 & \pm & 0.65 & 5.27 & \pm & 1.22 & 6.11 & \pm & 2.35 \\
\hline Tumor/Bone & 2.39 & \pm & 1.31 & 2.46 & \pm & 0.94 & 1.92 & \pm & 0.29 & 2.36 & \pm & 0.55 & 4.21 & \pm & 2.72 \\
\hline
\end{tabular}

Table S2-2. Tissue uptake (mean $\% \mathrm{ID} / \mathrm{g} \pm \mathrm{SD}$ ) of ${ }^{64} \mathrm{Cu}-\mathrm{NOTA}-\mathrm{V}$ ar3 administered via the lateral tail vein in female, BALB/c mice with orthotopic 4T1 breast cancer allografts implanted into the mammary fat pad. 


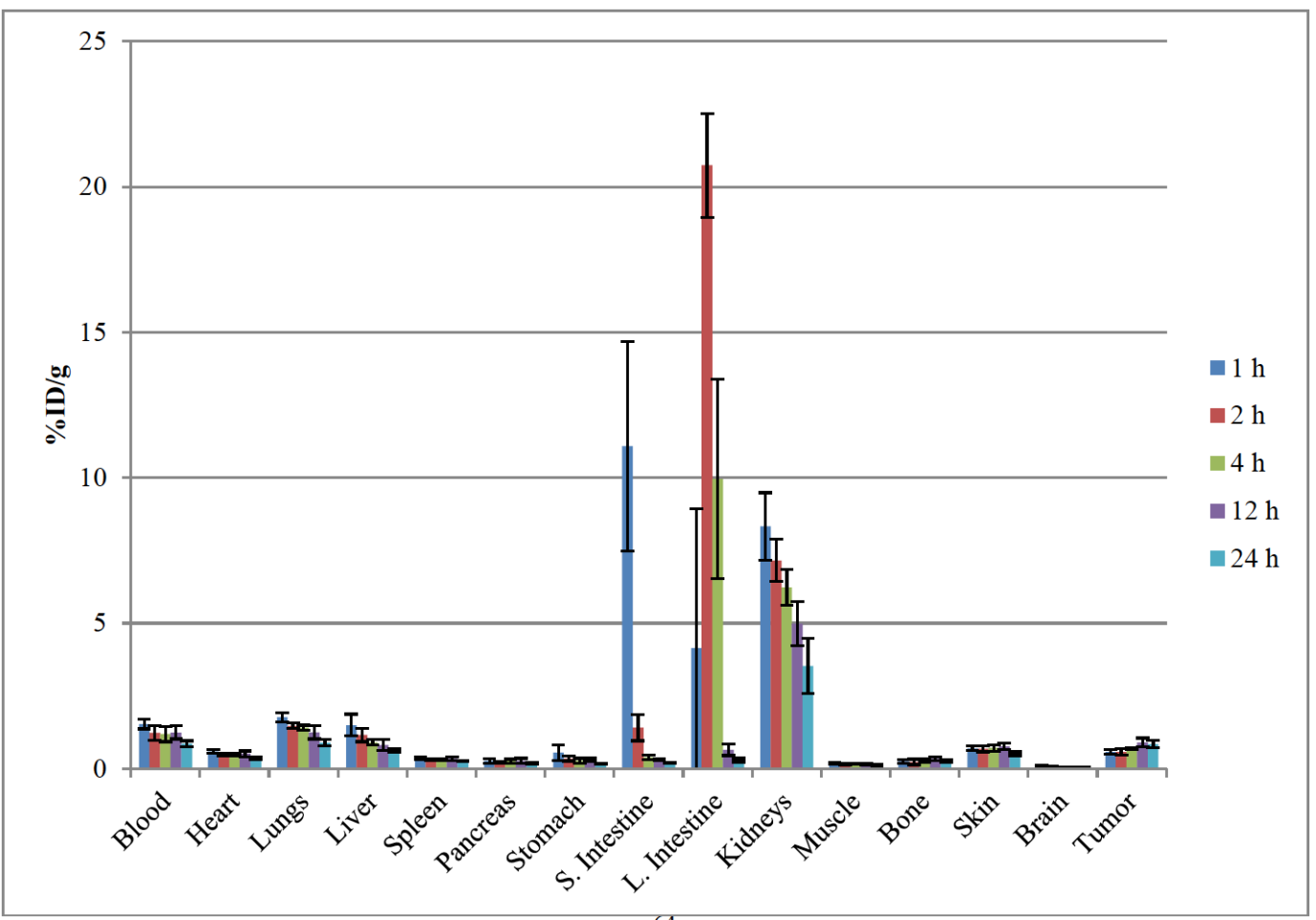

Fig. S2-1. Tissue uptake (mean $\% \mathrm{ID} / \mathrm{g} \pm \mathrm{SD}$ ) of ${ }^{64} \mathrm{Cu}-\mathrm{NOTA}-\mathrm{Var} 3(\mathrm{n}=5(4$ at $4 \mathrm{~h})$ ) administered via the lateral tail vein in female, BALB/c mice with orthotopic 4T1 breast cancer allografts implanted into the mammary fat pad full graph from text. 


\section{BIOSCAN}

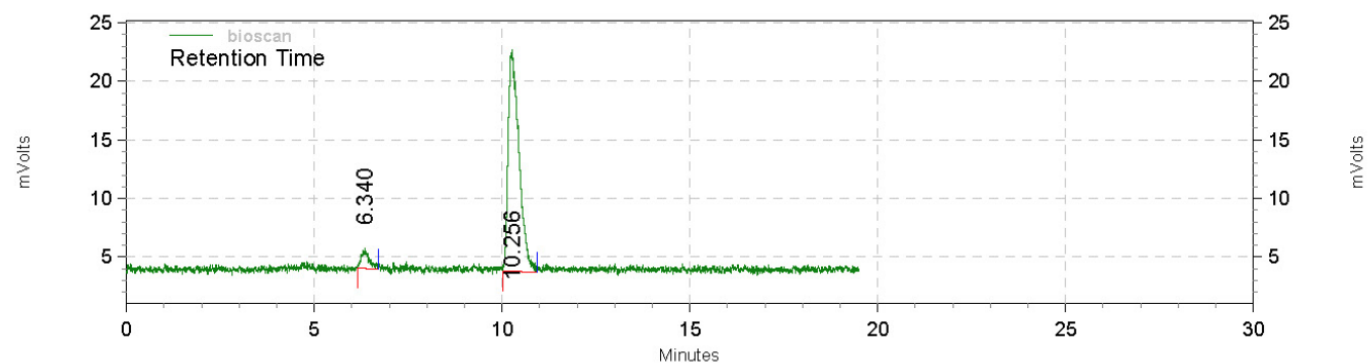

\begin{tabular}{rrrrrr}
$\begin{array}{l}\text { bioscan Results } \\
\text { Retention Time }\end{array}$ & Area & Area \% & Height & Height \% \\
\hline 6.340 & 23906 & 5.96 & 1735 & 8.39 \\
10.256 & 377018 & 94.04 & 18950 & 91.61 \\
\hline Totals & 400924 & 100.00 & 20685 & 100.00 \\
\hline
\end{tabular}

Fig. S2-2. HPLC Chromatogram of the Reconstituted ${ }^{64} \mathrm{Cu}-\mathrm{NOTA}-$ Var3 Injectate 
${ }^{64} \mathrm{Cu}-\mathrm{NOTA}-$ Var7

\begin{tabular}{|c|c|c|c|c|c|c|c|c|c|c|c|c|c|c|c|}
\hline \multirow{2}{*}{$\begin{array}{l}\text { Tissue } \\
\text { Blood }\end{array}$} & \multicolumn{3}{|c|}{$1 \mathrm{~h}(\mathrm{n}=4)$} & \multicolumn{3}{|c|}{$2 \mathrm{~h}(\mathrm{n}=4)$} & \multicolumn{3}{|c|}{$4 h(n=4)$} & \multicolumn{3}{|c|}{$12 \mathrm{~h}(\mathrm{n}=4)$} & \multicolumn{3}{|c|}{$24 \mathrm{~h}(\mathrm{n}=4)$} \\
\hline & 0.258 & \pm & 0.212 & 0.228 & \pm & 0.118 & 0.070 & \pm & 0.033 & 0.074 & \pm & 0.028 & 0.163 & \pm & 0.116 \\
\hline Heart & 0.037 & \pm & 0.006 & 0.030 & \pm & 0.004 & 0.035 & \pm & 0.004 & 0.031 & \pm & 0.009 & 0.028 & \pm & 0.005 \\
\hline Lungs & 0.252 & \pm & 0.010 & 0.289 & \pm & 0.074 & 0.267 & \pm & 0.059 & 0.206 & \pm & 0.059 & 0.142 & \pm & 0.020 \\
\hline Liver & 0.923 & \pm & 0.229 & 0.640 & \pm & 0.246 & 0.673 & \pm & 0.038 & 0.396 & \pm & 0.028 & 0.298 & + & 0.050 \\
\hline Spleen & 0.032 & \pm & 0.004 & 0.024 & \pm & 0.001 & 0.034 & \pm & 0.004 & 0.034 & \pm & 0.005 & 0.025 & \pm & 0.002 \\
\hline Pancreas & 0.023 & \pm & 0.005 & 0.018 & \pm & 0.006 & 0.032 & \pm & 0.014 & 0.026 & \pm & 0.009 & 0.018 & \pm & 0.004 \\
\hline Stomach & 0.081 & \pm & 0.017 & 0.070 & \pm & 0.024 & 0.133 & \pm & 0.026 & 0.085 & \pm & 0.006 & 0.052 & \pm & 0.016 \\
\hline S. intestine & 9.532 & \pm & 1.902 & 0.832 & \pm & 0.367 & 0.374 & \pm & 0.103 & 0.233 & \pm & 0.030 & 0.153 & \pm & 0.026 \\
\hline L. intestine & 0.300 & \pm & 0.072 & 12.452 & \pm & 1.188 & 5.608 & \pm & 0.658 & 0.201 & \pm & 0.026 & 0.265 & \pm & 0.103 \\
\hline Kidneys & 1.875 & \pm & 0.173 & 1.679 & \pm & 0.078 & 1.764 & \pm & 0.059 & 1.067 & \pm & 0.094 & 0.867 & \pm & 0.124 \\
\hline Muscle & 0.010 & \pm & 0.005 & 0.015 & \pm & 0.001 & 0.012 & \pm & 0.006 & 0.008 & \pm & 0.003 & 0.007 & \pm & 0.002 \\
\hline Bone & 0.003 & \pm & 0.002 & 0.002 & \pm & 0.001 & 0.011 & \pm & 0.002 & 0.008 & \pm & 0.003 & 0.004 & \pm & 0.002 \\
\hline Skin & 0.028 & \pm & 0.014 & 0.041 & \pm & 0.011 & 0.039 & \pm & 0.004 & 0.043 & \pm & 0.014 & 0.038 & \pm & 0.005 \\
\hline Brain & 0.030 & \pm & 0.007 & 0.019 & \pm & 0.002 & 0.016 & \pm & 0.002 & 0.010 & \pm & 0.004 & 0.011 & \pm & 0.003 \\
\hline Tumor & 0.074 & \pm & 0.029 & 0.063 & \pm & 0.017 & 0.094 & \pm & 0.017 & 0.158 & \pm & 0.066 & 0.161 & \pm & 0.036 \\
\hline
\end{tabular}

Table S3-1. Tissue uptake (mean $\%$ ID \pm SD) of ${ }^{64} \mathrm{Cu}-\mathrm{NOTA}-\mathrm{var} 7$ administered via

the lateral tail vein in female, BALB/c mice with orthotopic 4T1 breast cancer allografts implanted into the mammary fat pad. 


\begin{tabular}{|c|c|c|c|c|c|c|c|c|c|c|c|c|c|c|c|}
\hline \multirow{2}{*}{$\begin{array}{l}\text { Tissue } \\
\text { Blood }\end{array}$} & \multicolumn{3}{|c|}{$1 \mathrm{~h}(\mathrm{n}=4)$} & \multicolumn{3}{|c|}{$2 h(n=4)$} & \multicolumn{3}{|c|}{$4 h(n=4)$} & \multicolumn{3}{|c|}{$12 h(n=4)$} & \multicolumn{3}{|c|}{$24 \mathrm{~h}(\mathrm{n}=4)$} \\
\hline & 0.988 & \pm & 0.172 & 0.804 & \pm & 0.078 & 0.833 & \pm & 0.068 & 0.759 & \pm & 0.113 & 0.615 & \pm & 0.083 \\
\hline Heart & 0.333 & \pm & 0.055 & 0.316 & \pm & 0.042 & 0.353 & \pm & 0.041 & 0.307 & \pm & 0.036 & 0.282 & \pm & 0.075 \\
\hline Lungs & 1.094 & \pm & 0.153 & 0.979 & \pm & 0.104 & 0.996 & \pm & 0.066 & 0.904 & \pm & 0.088 & 0.666 & \pm & 0.107 \\
\hline Liver & 1.011 & \pm & 0.090 & 0.915 & \pm & 0.152 & 0.739 & \pm & 0.041 & 0.479 & \pm & 0.038 & 0.449 & \pm & 0.091 \\
\hline Spleen & 0.217 & \pm & 0.036 & 0.215 & \pm & 0.020 & 0.279 & \pm & 0.016 & 0.213 & \pm & 0.031 & 0.175 & \pm & 0.030 \\
\hline Pancreas & 0.182 & \pm & 0.040 & 0.150 & \pm & 0.032 & 0.198 & \pm & 0.043 & 0.182 & \pm & 0.029 & 0.122 & \pm & 0.019 \\
\hline Stomach & 0.324 & \pm & 0.062 & 0.299 & \pm & 0.107 & 0.300 & \pm & 0.076 & 0.078 & \pm & 0.015 & 0.200 & \pm & 0.070 \\
\hline S. intestine & 9.167 & \pm & 2.676 & 0.935 & \pm & 0.329 & 0.307 & \pm & 0.077 & 0.158 & \pm & 0.012 & 0.179 & \pm & 0.044 \\
\hline L. intestine & 0.461 & \pm & 0.081 & 18.811 & \pm & 2.211 & 7.782 & \pm & 0.609 & 0.255 & \pm & 0.043 & 0.368 & \pm & 0.098 \\
\hline Kidneys & 6.760 & \pm & 0.950 & 6.065 & \pm & 0.619 & 6.790 & \pm & 0.461 & 4.143 & \pm & 0.512 & 3.410 & \pm & 0.525 \\
\hline Muscle & 0.123 & \pm & 0.018 & 0.141 & \pm & 0.018 & 0.118 & \pm & 0.025 & 0.104 & \pm & 0.016 & 0.101 & \pm & 0.020 \\
\hline Bone & 0.141 & \pm & 0.055 & 0.099 & \pm & 0.053 & 0.229 & \pm & 0.093 & 0.247 & \pm & 0.039 & 0.136 & \pm & 0.064 \\
\hline Skin & 0.490 & \pm & 0.053 & 0.497 & \pm & 0.060 & 0.563 & \pm & 0.145 & 0.566 & \pm & 0.092 & 0.510 & \pm & 0.120 \\
\hline Brain & 0.071 & \pm & 0.016 & 0.053 & \pm & 0.003 & 0.042 & \pm & 0.004 & 0.032 & \pm & 0.007 & 0.032 & \pm & 0.005 \\
\hline Tumor & 0.366 & \pm & 0.034 & 0.414 & \pm & 0.037 & 0.546 & \pm & 0.075 & 0.673 & \pm & 0.078 & 0.693 & \pm & 0.040 \\
\hline \multicolumn{16}{|c|}{ Tumor-to-tissue ratios (rel. u.) } \\
\hline Tumor/Blood & 0.371 & \pm & 0.073 & 0.515 & \pm & 0.068 & 0.655 & \pm & 0.105 & 0.886 & \pm & 0.168 & 1.126 & \pm & 0.165 \\
\hline Tumor/Liver & 0.362 & \pm & 0.047 & 0.452 & \pm & 0.086 & 0.738 & \pm & 0.110 & 1.403 & \pm & 0.198 & 1.542 & \pm & 0.325 \\
\hline Tumor/S. I. & 0.040 & \pm & 0.012 & 0.443 & \pm & 0.161 & 1.780 & \pm & 0.510 & 4.259 & \pm & 0.599 & 3.878 & \pm & 0.972 \\
\hline Tumor/L. I. & 0.794 & \pm & 0.158 & 0.022 & \pm & 0.003 & 0.070 & \pm & 0.011 & 2.642 & \pm & 0.540 & 1.883 & \pm & 0.514 \\
\hline Tumor/Kidney & 0.054 & \pm & 0.009 & 0.068 & \pm & 0.009 & 0.080 & \pm & 0.012 & 0.162 & \pm & 0.028 & 0.203 & \pm & 0.033 \\
\hline Tumor/Muscle & 2.966 & \pm & 0.518 & 2.947 & \pm & 0.465 & 4.618 & \pm & 1.167 & 6.444 & \pm & 1.231 & 6.878 & \pm & 1.430 \\
\hline Tumor/Bone & 2.598 & \pm & 1.040 & 4.193 & \pm & 2.268 & 2.388 & \pm & 1.030 & 2.727 & \pm & 0.536 & 5.087 & \pm & 2.404 \\
\hline
\end{tabular}

Table S3-2. Tissue uptake (mean $\% \mathrm{ID} / \mathrm{g} \pm \mathrm{SD}$ ) of ${ }^{64} \mathrm{Cu}-\mathrm{NOTA}-\mathrm{Var} 7$ administered via the lateral tail vein in female, BALB/c mice with orthotopic 4T1 breast cancer allografts implanted into the mammary fat pad. 


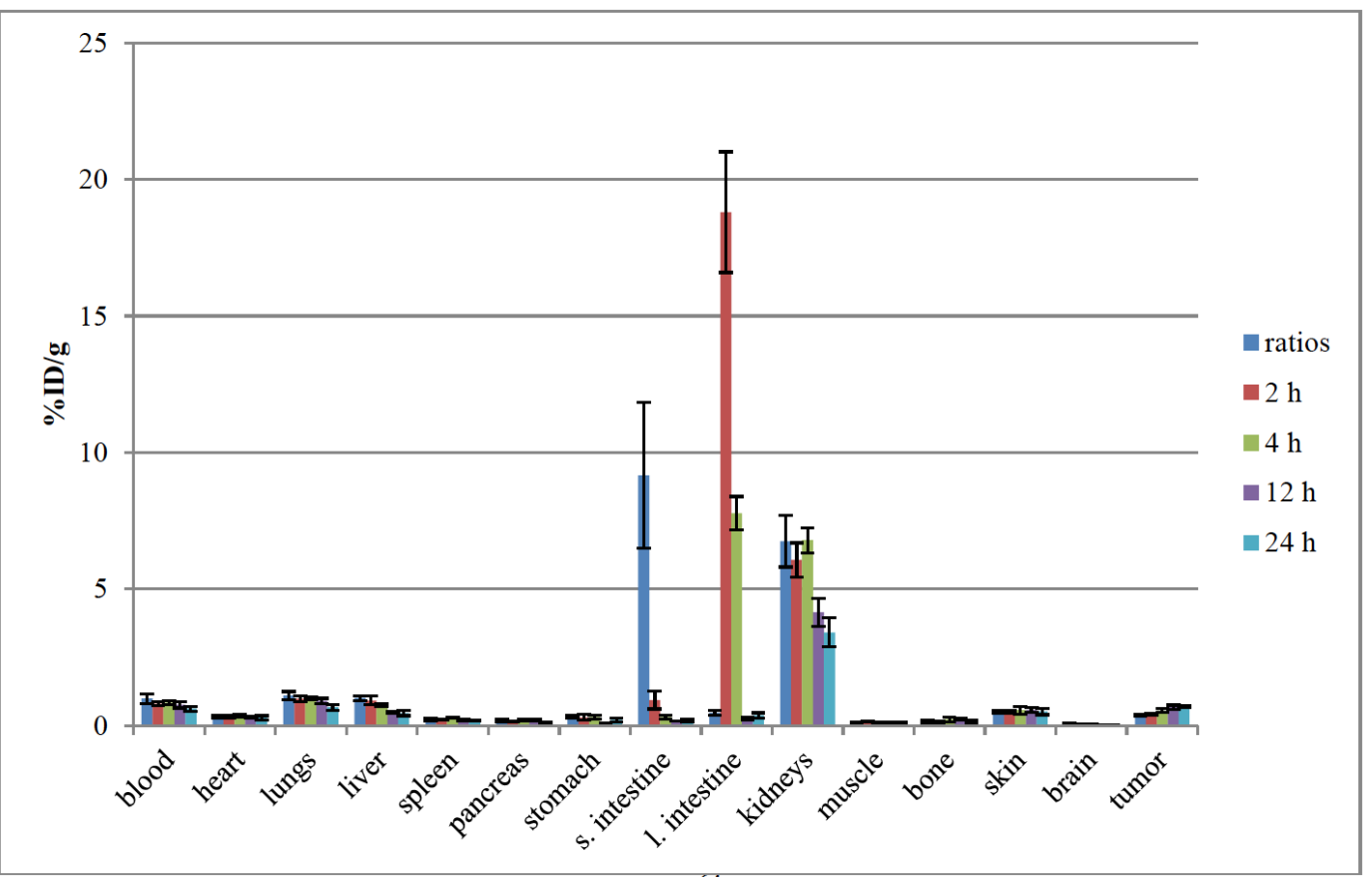

Fig. S3-1. Tissue uptake (mean $\% \mathrm{ID} / \mathrm{g} \pm \mathrm{SD})$ of ${ }^{64} \mathrm{Cu}-\mathrm{NOTA}-\mathrm{var} 7(\mathrm{n}=4)$ administered via the lateral tail vein in female, BALB/c mice with orthotopic $4 \mathrm{~T} 1$ breast cancer allografts implanted into the mammary fat pad full graph from text. 


\section{BIOSCAN}

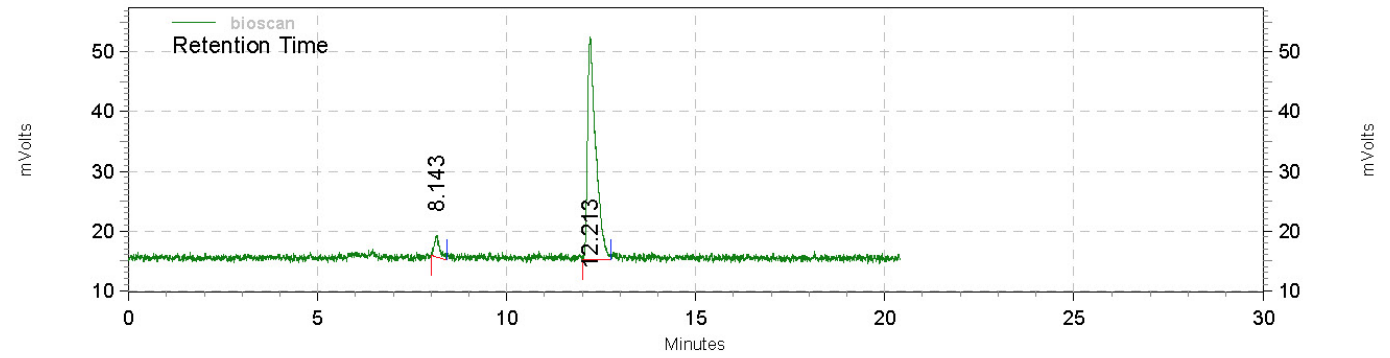

\begin{tabular}{rrrrrr}
$\begin{array}{r}\text { bioscan Results } \\
\text { Retention Time }\end{array}$ & Area & Area \% & Height & Height \% \\
\hline 8.143 & 36720 & 6.07 & 3580 & 8.72 \\
12.213 & 567879 & 93.93 & 37462 & 91.28 \\
\hline \multicolumn{7}{|c|}{} & & & & \\
\hline Totals & 604599 & 100.00 & 41042 & 100.00 \\
\hline
\end{tabular}

Fig. S3-2. HPLC Chromatogram of the Reconstituted ${ }^{64} \mathrm{Cu}-\mathrm{NOTA}-\mathrm{Var} 7$ Injectate 
${ }^{18}$ F-AIF-NOTA-WT

\begin{tabular}{lcccccccccccc}
\hline Tissue & \multicolumn{2}{c}{$\mathbf{0 . 5} \mathbf{~ h}(\mathbf{n}=\mathbf{5})$} & \multicolumn{2}{c}{$\mathbf{1} \mathbf{h}(\mathbf{n}=\mathbf{5})$} & \multicolumn{2}{c}{$\mathbf{2} \mathbf{~ h}(\mathbf{n}=\mathbf{5})$} & \multicolumn{3}{c}{$\mathbf{4} \mathbf{h}(\mathbf{n}=\mathbf{5})$} \\
\hline Blood & 1.319 & \pm & 0.188 & 1.112 & \pm & 0.131 & 0.625 & \pm & 0.423 & 0.637 & \pm & 0.462 \\
Heart & 0.093 & \pm & 0.015 & 0.103 & \pm & 0.031 & 0.092 & \pm & 0.025 & 0.061 & \pm & 0.014 \\
Lungs & 0.525 & \pm & 0.082 & 0.533 & \pm & 0.125 & 0.468 & \pm & 0.114 & 0.485 & \pm & 0.062 \\
Liver & 1.681 & \pm & 0.216 & 1.218 & \pm & 0.260 & 1.238 & \pm & 0.281 & 0.794 & \pm & 0.142 \\
Spleen & 0.069 & \pm & 0.009 & 0.074 & \pm & 0.010 & 0.078 & \pm & 0.006 & 0.068 & \pm & 0.014 \\
Pancreas & 0.049 & \pm & 0.018 & 0.043 & \pm & 0.008 & 0.049 & \pm & 0.009 & 0.044 & \pm & 0.009 \\
Stomach & 0.108 & \pm & 0.006 & 0.129 & \pm & 0.033 & 0.118 & \pm & 0.009 & 0.141 & \pm & 0.027 \\
S. intestine & 12.837 & \pm & 0.988 & 13.737 & \pm & 1.399 & 1.120 & \pm & 0.148 & 0.738 & \pm & 0.243 \\
L. intestine & 0.197 & \pm & 0.039 & 0.266 & \pm & 0.079 & 16.243 & \pm & 1.989 & 16.258 & \pm & 1.370 \\
Kidneys & 5.518 & \pm & 0.849 & 3.471 & \pm & 0.123 & 2.861 & \pm & 0.052 & 2.289 & \pm & 0.250 \\
Muscle & 0.040 & \pm & 0.007 & 0.038 & \pm & 0.005 & 0.036 & \pm & 0.008 & 0.031 & \pm & 0.011 \\
Bone & 0.025 & \pm & 0.011 & 0.013 & \pm & 0.003 & 0.034 & \pm & 0.018 & 0.046 & \pm & 0.004 \\
Skin & 0.114 & \pm & 0.051 & 0.129 & \pm & 0.035 & 0.099 & \pm & 0.029 & 0.105 & \pm & 0.003 \\
Brain & 0.087 & \pm & 0.007 & 0.063 & \pm & 0.009 & 0.052 & \pm & 0.006 & 0.039 & \pm & 0.008 \\
Tumor & 0.159 & \pm & 0.045 & 0.163 & \pm & 0.042 & 0.175 & \pm & 0.028 & 0.187 & \pm & 0.060 \\
\hline
\end{tabular}

Table S4-1. Tissue uptake (mean $\%$ ID \pm SD) of ${ }^{18} \mathrm{~F}-\mathrm{AlF}-\mathrm{NOTA}-\mathrm{WT}$ administered via the lateral tail vein in female, BALB/c mice with orthotopic 4T1 breast cancer allografts implanted into the mammary fat pad. 


\begin{tabular}{|c|c|c|c|c|c|c|c|c|c|c|c|c|}
\hline \multirow{2}{*}{$\begin{array}{l}\text { Tissue } \\
\text { Blood }\end{array}$} & \multicolumn{3}{|c|}{$0.5 h(n=5)$} & \multicolumn{3}{|c|}{$1 \mathrm{~h}(\mathrm{n}=5)$} & \multicolumn{3}{|c|}{$2 h(n=5)$} & \multicolumn{3}{|c|}{$4 h(n=5)$} \\
\hline & 2.305 & \pm & 0.308 & 2.034 & \pm & 0.288 & 2.096 & \pm & 0.250 & 1.978 & \pm & 0.192 \\
\hline Heart & 0.919 & \pm & 0.105 & 0.884 & \pm & 0.167 & 0.856 & \pm & 0.091 & 0.664 & \pm & 0.119 \\
\hline Lungs & 2.353 & \pm & 0.250 & 2.288 & \pm & 0.072 & 2.186 & \pm & 0.273 & 2.049 & \pm & 0.211 \\
\hline Liver & 1.964 & \pm & 0.473 & 1.163 & \pm & 0.166 & 1.158 & \pm & 0.189 & 0.973 & \pm & 0.213 \\
\hline Spleen & 0.482 & \pm & 0.080 & 0.423 & \pm & 0.042 & 0.418 & \pm & 0.046 & 0.434 & \pm & 0.058 \\
\hline Pancreas & 0.359 & \pm & 0.089 & 0.318 & \pm & 0.018 & 0.355 & \pm & 0.062 & 0.324 & \pm & 0.059 \\
\hline Stomach & 0.406 & \pm & 0.114 & 0.364 & \pm & 0.087 & 0.442 & \pm & 0.077 & 0.518 & \pm & 0.152 \\
\hline S. intestine & 12.969 & \pm & 1.568 & 13.510 & \pm & 0.999 & 1.080 & \pm & 0.198 & 0.820 & \pm & 0.245 \\
\hline L. intestine & 0.264 & \pm & 0.037 & 0.368 & \pm & 0.126 & 24.375 & \pm & 2.182 & 24.064 & \pm & 2.848 \\
\hline Kidneys & 18.931 & \pm & 3.456 & 12.308 & \pm & 0.760 & 10.247 & \pm & 0.914 & 8.550 & \pm & 1.138 \\
\hline Muscle & 0.360 & \pm & 0.070 & 0.315 & \pm & 0.034 & 0.349 & \pm & 0.073 & 0.283 & \pm & 0.041 \\
\hline Bone & 0.635 & \pm & 0.181 & 0.627 & \pm & 0.185 & 0.702 & \pm & 0.166 & 0.671 & \pm & 0.305 \\
\hline Skin & 1.256 & \pm & 0.240 & 1.138 & \pm & 0.093 & 1.027 & \pm & 0.178 & 1.032 & \pm & 0.133 \\
\hline Brain & 0.218 & \pm & 0.018 & 0.165 & \pm & 0.015 & 0.135 & \pm & 0.009 & 0.100 & \pm & 0.015 \\
\hline Tumor & 0.995 & \pm & 0.242 & 0.930 & \pm & 0.129 & 0.944 & \pm & 0.142 & 1.005 & \pm & 0.232 \\
\hline \multicolumn{13}{|c|}{ Tumor-to-tissue ratios (rel. u.) } \\
\hline Tumor/Blood & 0.43 & \pm & 0.12 & 0.46 & \pm & 0.09 & 0.45 & \pm & 0.09 & 0.51 & \pm & 0.13 \\
\hline Tumor/Liver & 0.51 & \pm & 0.17 & 0.80 & \pm & 0.16 & 0.82 & \pm & 0.18 & 1.03 & \pm & 0.33 \\
\hline Tumor/S. I. & 0.08 & \pm & 0.02 & 0.07 & \pm & 0.01 & 0.87 & \pm & 0.21 & 1.22 & \pm & 0.46 \\
\hline Tumor/L. I. & 3.77 & \pm & 1.06 & 2.52 & \pm & 0.93 & 0.04 & \pm & 0.01 & 0.04 & \pm & 0.01 \\
\hline Tumor/Kidney & 0.05 & \pm & 0.02 & 0.08 & \pm & 0.01 & 0.092 & \pm & 0.016 & 0.12 & \pm & 0.03 \\
\hline Tumor/Muscle & 2.76 & \pm & 0.86 & 2.96 & \pm & 0.52 & 2.70 & \pm & 0.70 & 3.54 & \pm & 0.97 \\
\hline Tumor/Bone & 1.57 & \pm & 0.59 & 1.48 & \pm & 0.48 & 1.345 & \pm & 0.377 & 1.50 & \pm & 0.76 \\
\hline
\end{tabular}

Table S4-2. Tissue uptake (mean $\% \mathrm{ID} / \mathrm{g} \pm \mathrm{SD}$ ) of ${ }^{18} \mathrm{~F}-\mathrm{AlF}-\mathrm{NOTA}-\mathrm{WT}$ administered via the lateral tail vein in female, BALB/c mice with orthotopic 4T1 breast cancer allografts implanted into the mammary fat pad. 


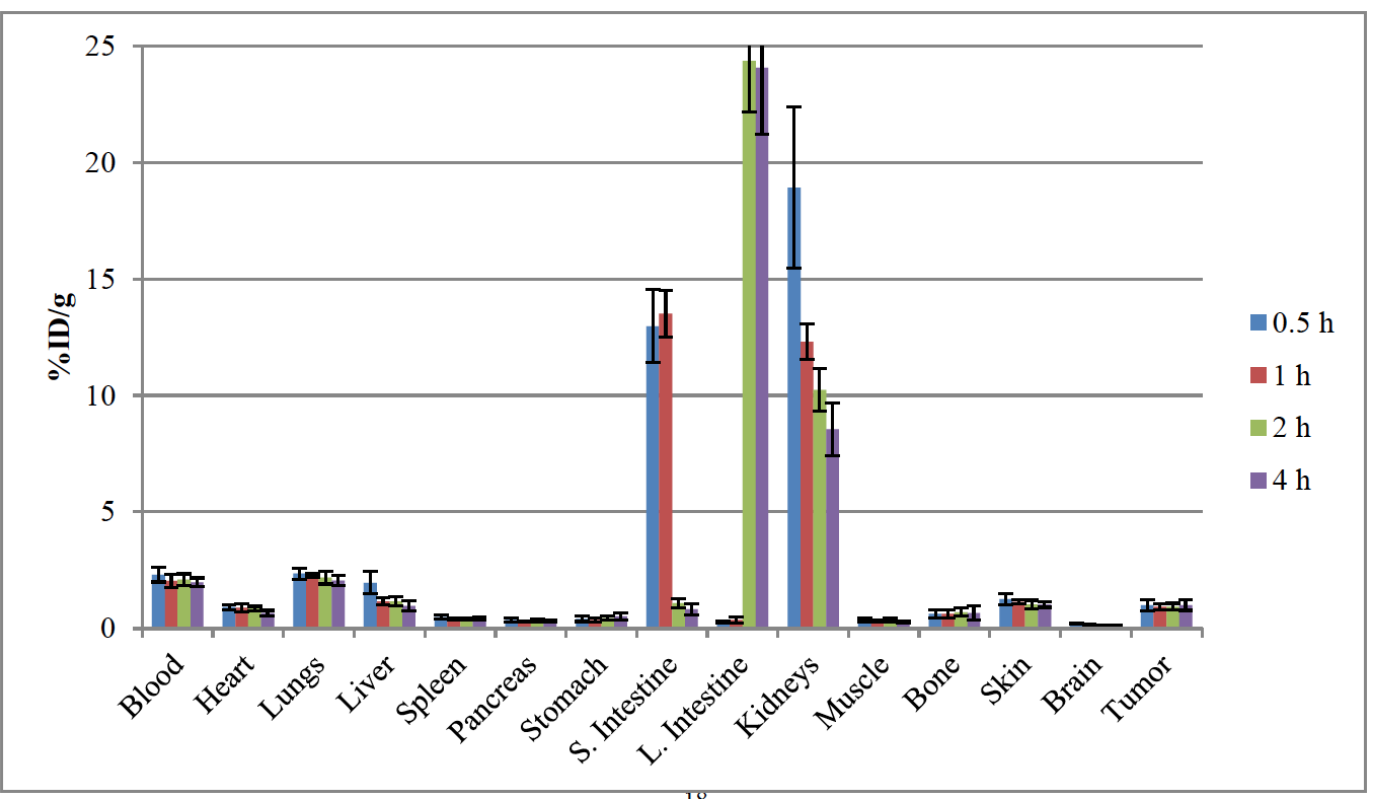

Fig. S4-1. Tissue uptake (mean $\% \mathrm{ID} / \mathrm{g} \pm \mathrm{SD}$ ) of ${ }^{18} \mathrm{~F}-\mathrm{AlF}-\mathrm{NOTA}-\mathrm{WT}(\mathrm{n}=5)$ administered via the lateral tail vein in female, BALB/c mice with orthotopic 4T1 breast cancer allografts implanted into the mammary fat pad full graph from text. 


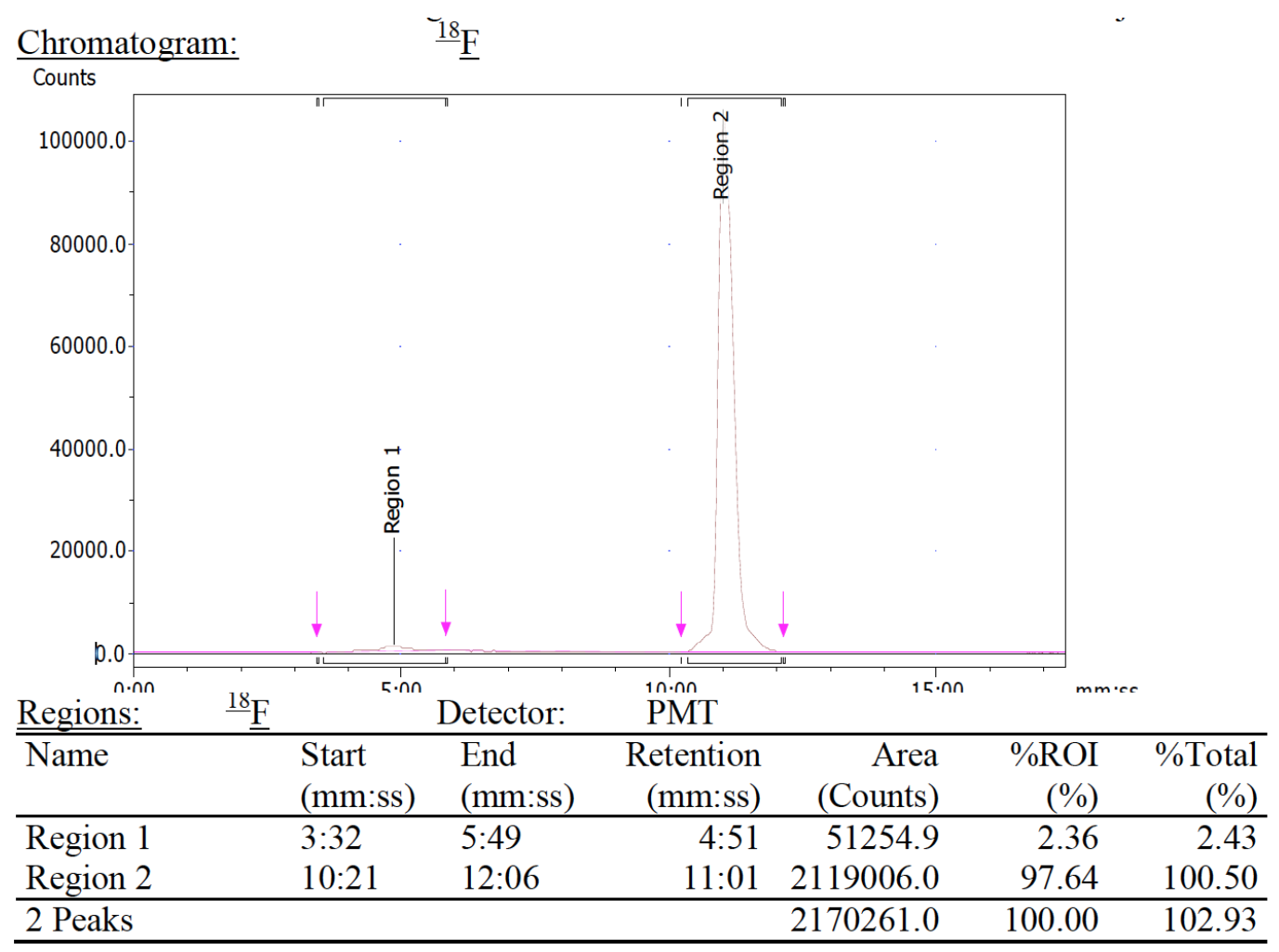

Fig. S4-2. HPLC Chromatogram of the Reconstituted ${ }^{18}$ F-AIF-NOTA-WT Injectate 


\section{${ }^{18}$ F-AIF-NOTA-Var3}

\begin{tabular}{|c|c|c|c|c|c|c|c|c|c|c|c|c|}
\hline \multirow{2}{*}{$\begin{array}{l}\text { Tissue } \\
\text { Blood }\end{array}$} & \multicolumn{3}{|c|}{$0.5 \mathrm{~h}(\mathrm{n}=5)$} & \multicolumn{3}{|c|}{$1 \mathrm{~h}(\mathrm{n}=5)$} & \multicolumn{3}{|c|}{$2 h(n=5)$} & \multicolumn{3}{|c|}{$4 h(n=4)$} \\
\hline & 0.866 & \pm & 0.319 & 0.481 & \pm & 0.473 & 0.337 & \pm & 0.229 & 1.083 & \pm & 0.234 \\
\hline Heart & 0.117 & \pm & 0.020 & 0.095 & \pm & 0.019 & 0.093 & \pm & 0.022 & 0.078 & \pm & 0.006 \\
\hline Lungs & 0.815 & \pm & 0.339 & 0.573 & \pm & 0.157 & 0.588 & \pm & 0.197 & 0.543 & \pm & 0.107 \\
\hline Liver & 2.398 & \pm & 1.056 & 1.627 & \pm & 0.382 & 1.091 & \pm & 0.215 & 0.779 & \pm & 0.050 \\
\hline Spleen & 0.096 & \pm & 0.009 & 0.076 & \pm & 0.007 & 0.055 & \pm & 0.014 & 0.070 & \pm & 0.008 \\
\hline Pancreas & 0.116 & \pm & 0.115 & 0.047 & \pm & 0.008 & 0.047 & \pm & 0.014 & 0.061 & \pm & 0.030 \\
\hline Stom & 0.235 & \pm & 0.049 & 0.149 & \pm & 0.029 & 0.132 & \pm & 0.015 & 0.130 & \pm & 0.018 \\
\hline S. inte & 12.015 & \pm & 1.355 & 13.855 & \pm & 1.696 & 2.964 & \pm & 0.687 & 1.078 & \pm & 0.208 \\
\hline L. intestine & 0.326 & \pm & 0.101 & 1.350 & \pm & 1.253 & 13.535 & \pm & 0.993 & 14.054 & \pm & 1.192 \\
\hline Kidneys & 6.242 & \pm & 0.722 & 3.442 & \pm & 0.594 & 2.748 & \pm & 0.105 & 2.236 & \pm & 0.141 \\
\hline Muscle & 0.041 & \pm & 0.014 & 0.044 & \pm & 0.012 & 0.033 & \pm & 0.011 & 0.028 & \pm & 0.004 \\
\hline Bone & 0.027 & \pm & 0.019 & 0.022 & \pm & 0.014 & 0.017 & \pm & 0.014 & 0.016 & \pm & 0.009 \\
\hline Skin & 0.171 & \pm & 0.030 & 0.180 & \pm & 0.018 & 0.161 & \pm & 0.040 & 0.106 & \pm & 0.041 \\
\hline Brain & 0.080 & \pm & 0.014 & 0.063 & \pm & 0.006 & 0.054 & \pm & 0.007 & 0.041 & \pm & 0.011 \\
\hline Tumor & 0.188 & \pm & 0.033 & 0.164 & \pm & 0.025 & 0.134 & \pm & 0.035 & 0.216 & \pm & 0.047 \\
\hline
\end{tabular}

Table S5-1. Tissue uptake (mean $\%$ ID \pm SD) of ${ }^{18}$ F-AlF-NOTA-Var3 administered via the lateral tail vein in female, BALB/c mice with orthotopic $4 \mathrm{~T} 1$ breast cancer allografts implanted into the mammary fat pad. 


\begin{tabular}{|c|c|c|c|c|c|c|c|c|c|c|c|c|}
\hline \multirow{2}{*}{$\begin{array}{l}\text { Tissue } \\
\text { Blood }\end{array}$} & \multicolumn{3}{|c|}{$0.5 \mathrm{~h}(\mathrm{n}=5)$} & \multicolumn{3}{|c|}{$1 \mathrm{~h}(\mathrm{n}=5)$} & \multicolumn{3}{|c|}{$2 h(n=5)$} & \multicolumn{3}{|c|}{$4 h(n=4)$} \\
\hline & 5.778 & \pm & 2.950 & 2.851 & \pm & 0.208 & 1.998 & \pm & 0.319 & 2.201 & \pm & 0.374 \\
\hline Heart & 1.128 & \pm & 0.154 & 1.024 & \pm & 0.112 & 0.879 & \pm & 0.098 & 0.775 & \pm & 0.079 \\
\hline Lungs & 3.470 & \pm & 0.434 & 2.567 & \pm & 0.497 & 2.375 & \pm & 0.279 & 2.217 & \pm & 0.380 \\
\hline Liver & 2.960 & \pm & 0.390 & 1.605 & \pm & 0.396 & 1.302 & \pm & 0.332 & 0.923 & \pm & 0.153 \\
\hline Spleen & 0.706 & \pm & 0.106 & 0.554 & \pm & 0.053 & 0.440 & \pm & 0.148 & 0.469 & \pm & 0.093 \\
\hline Pancreas & 0.754 & \pm & 0.414 & 0.405 & \pm & 0.070 & 0.380 & \pm & 0.140 & 0.332 & \pm & 0.020 \\
\hline Stomach & 1.034 & \pm & 0.138 & 0.648 & \pm & 0.300 & 0.586 & \pm & 0.096 & 0.482 & \pm & 0.223 \\
\hline S. intestine & 10.125 & \pm & 1.224 & 12.675 & \pm & 2.221 & 2.671 & \pm & 0.830 & 0.927 & \pm & 0.152 \\
\hline L. intestine & 0.434 & \pm & 0.077 & 1.879 & \pm & 1.637 & 19.737 & \pm & 2.432 & 22.047 & \pm & 2.461 \\
\hline Kidneys & 21.180 & \pm & 3.755 & 11.398 & \pm & 1.054 & 10.166 & \pm & 1.290 & 8.237 & \pm & 1.106 \\
\hline Muscle & 0.413 & \pm & 0.040 & 0.391 & \pm & 0.105 & 0.316 & \pm & 0.040 & 0.282 & \pm & 0.055 \\
\hline Bone & 0.626 & \pm & 0.177 & 0.547 & \pm & 0.127 & 0.516 & \pm & 0.063 & 0.540 & \pm & 0.119 \\
\hline Skin & 1.926 & \pm & 0.399 & 1.557 & \pm & 0.270 & 1.411 & \pm & 0.163 & 1.126 & \pm & 0.115 \\
\hline Brain & 0.222 & \pm & 0.033 & 0.162 & \pm & 0.012 & 0.133 & \pm & 0.017 & 0.107 & \pm & 0.023 \\
\hline Tumor & 1.142 & \pm & 0.244 & 0.913 & \pm & 0.046 & 0.802 & \pm & 0.129 & 0.923 & \pm & 0.119 \\
\hline \multicolumn{13}{|c|}{ Tumor-to-tissue ratios (rel. u.) } \\
\hline Tumor/Blood & 0.198 & \pm & 0.109 & 0.320 & \pm & 0.028 & 0.401 & \pm & 0.091 & 0.419 & \pm & 0.090 \\
\hline Tumor/Liver & 0.386 & \pm & 0.097 & 0.569 & \pm & 0.143 & 0.615 & \pm & 0.186 & 1.000 & \pm & 0.211 \\
\hline Tumor/S. I. & 0.113 & \pm & 0.028 & 0.072 & \pm & 0.013 & 0.300 & \pm & 0.105 & 0.996 & \pm & 0.208 \\
\hline Tumor/L. I. & 2.631 & \pm & 0.733 & 0.486 & \pm & 0.424 & 0.041 & \pm & 0.008 & 0.042 & \pm & 0.007 \\
\hline Tumor/Kidney & 0.054 & \pm & 0.015 & 0.080 & \pm & 0.008 & 0.079 & \pm & 0.016 & 0.112 & \pm & 0.021 \\
\hline Tumor/Muscle & 2.763 & \pm & 0.648 & 2.334 & \pm & 0.635 & 2.535 & \pm & 0.520 & 3.277 & \pm & 0.765 \\
\hline Tumor/Bone & 1.823 & \pm & 0.646 & 1.668 & \pm & 0.397 & 1.554 & \pm & 0.315 & 1.709 & \pm & 0.436 \\
\hline
\end{tabular}

Table S5-2. Tissue uptake (mean $\% \mathrm{ID} / \mathrm{g} \pm \mathrm{SD}$ ) of ${ }^{18} \mathrm{~F}-\mathrm{AlF}-\mathrm{NOTA}-\mathrm{V}$ ar3 administered via the lateral tail vein in female, BALB/c mice with orthotopic $4 \mathrm{~T} 1$ breast cancer allografts implanted into the mammary fat pad. 


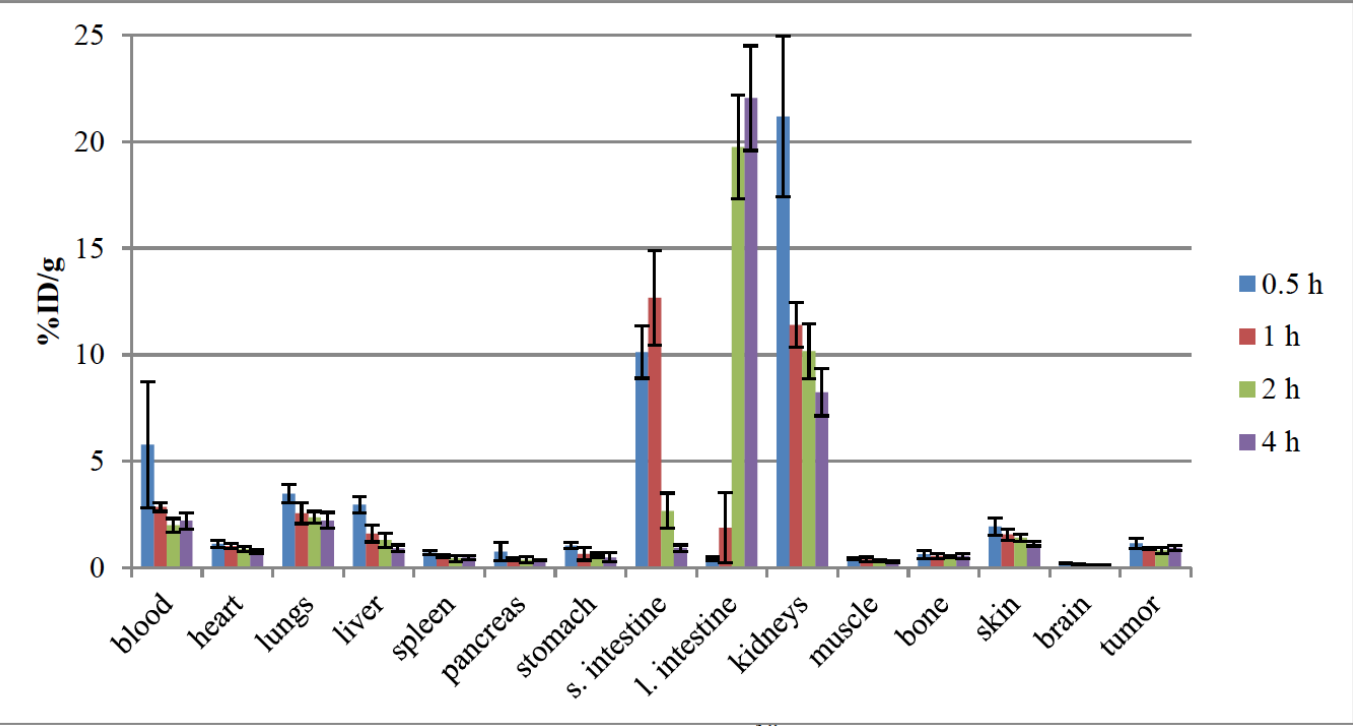

Fig. S5-1. Tissue uptake (mean $\% \mathrm{ID} / \mathrm{g} \pm \mathrm{SD}$ ) of ${ }^{18} \mathrm{~F}-\mathrm{AlF}-\mathrm{NOTA}-\operatorname{Var} 3(\mathrm{n}=5$ (4 at 4 h)) administered via the lateral tail vein in female, BALB/c mice with orthotopic 4T1 breast cancer allografts implanted into the mammary fat pad full graph from text. 


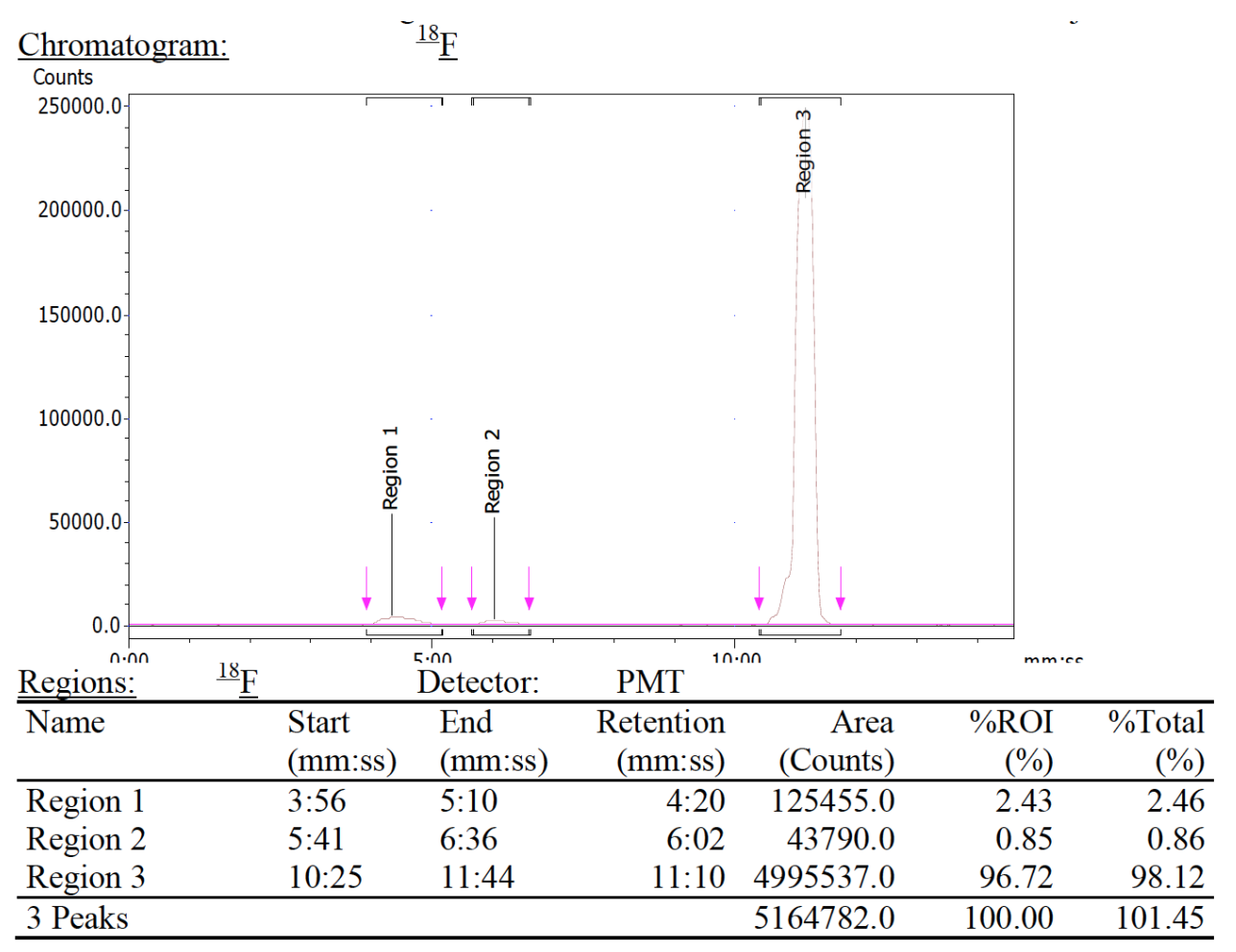

Fig. S5-2. HPLC Chromatogram of the Reconstituted ${ }^{18}$ F-AlF-NOTA-Var3 Injectate 


\section{${ }^{18}$ F-AIF-NOTA-Var7}

\begin{tabular}{|c|c|c|c|c|c|c|c|c|c|c|c|c|}
\hline Tissue & \multicolumn{3}{|c|}{$0.5 \mathrm{~h}(\mathrm{n}=5)$} & \multicolumn{3}{|c|}{$1 \mathrm{~h}(\mathrm{n}=5)$} & \multicolumn{3}{|c|}{$2 h(n=5)$} & \multicolumn{3}{|c|}{$4 h(n=5)$} \\
\hline Blood & 0.840 & \pm & 0.323 & 0.523 & \pm & 0.262 & 0.206 & \pm & 0.126 & 0.149 & \pm & 0.111 \\
\hline Heart & 0.105 & \pm & 0.015 & 0.101 & \pm & 0.038 & 0.091 & \pm & 0.022 & 0.076 & \pm & 0.012 \\
\hline Lungs & 0.625 & \pm & 0.122 & 0.555 & \pm & 0.304 & 0.485 & \pm & 0.196 & 0.593 & \pm & 0.054 \\
\hline Liver & 1.258 & \pm & 0.785 & 1.083 & \pm & 0.384 & 1.043 & \pm & 0.440 & 0.863 & \pm & 0.132 \\
\hline Spleen & 0.078 & \pm & 0.015 & 0.070 & \pm & 0.014 & 0.070 & \pm & 0.025 & 0.077 & \pm & 0.014 \\
\hline Pancreas & 0.053 & \pm & 0.013 & 0.042 & \pm & 0.008 & 0.055 & \pm & 0.014 & 0.054 & \pm & 0.015 \\
\hline Stomach & 0.163 & \pm & 0.036 & 0.127 & \pm & 0.039 & 0.136 & \pm & 0.013 & 0.167 & \pm & 0.052 \\
\hline S. intestine & 13.273 & \pm & 1.269 & 11.429 & \pm & 5.124 & 4.863 & \pm & 2.873 & 0.905 & \pm & 0.293 \\
\hline L. intestine & 0.207 & \pm & 0.050 & 1.006 & \pm & 1.208 & 10.930 & \pm & 2.777 & 13.783 & \pm & 4.870 \\
\hline Kidneys & 4.639 & \pm & 0.460 & 2.934 & \pm & 0.361 & 2.360 & \pm & 0.178 & 2.147 & \pm & 0.138 \\
\hline Muscle & 0.035 & \pm & 0.008 & 0.024 & \pm & 0.004 & 0.031 & \pm & 0.012 & 0.031 & \pm & 0.007 \\
\hline Bone & 0.011 & \pm & 0.004 & 0.011 & \pm & 0.006 & 0.014 & \pm & 0.007 & 0.022 & \pm & 0.021 \\
\hline Skin & 0.106 & \pm & 0.024 & 0.091 & \pm & 0.026 & 0.121 & \pm & 0.037 & 0.128 & \pm & 0.027 \\
\hline Brain & 0.122 & \pm & 0.098 & 0.066 & \pm & 0.021 & 0.053 & \pm & 0.006 & 0.047 & \pm & 0.006 \\
\hline Tumor & 0.174 & \pm & 0.057 & 0.163 & \pm & 0.055 & 0.163 & \pm & 0.036 & 0.209 & \pm & 0.070 \\
\hline
\end{tabular}

Table S6-1. Tissue uptake (mean $\%$ ID \pm SD) of ${ }^{18}$ F-AlF-NOTA-Var7 administered via the lateral tail vein in female, BALB/c mice with orthotopic $4 \mathrm{~T} 1$ breast cancer allografts implanted into the mammary fat pad. 


\begin{tabular}{|c|c|c|c|c|c|c|c|c|c|c|c|c|}
\hline \multirow{2}{*}{$\begin{array}{l}\text { Tissue } \\
\text { Blood }\end{array}$} & \multicolumn{3}{|c|}{$0.5 \mathrm{~h}(\mathrm{n}=5)$} & \multicolumn{3}{|c|}{$1 \mathrm{~h}(\mathrm{n}=5)$} & \multicolumn{3}{|c|}{$2 h(n=5)$} & \multicolumn{3}{|c|}{$4 h(n=5)$} \\
\hline & 2.961 & \pm & 0.684 & 2.263 & \pm & 0.412 & 2.547 & \pm & 0.642 & 2.292 & \pm & 0.277 \\
\hline Heart & 0.994 & \pm & 0.167 & 0.885 & \pm & 0.247 & 0.918 & \pm & 0.195 & 0.775 & \pm & 0.056 \\
\hline Lungs & 2.440 & \pm & 0.351 & 2.508 & \pm & 0.683 & 2.532 & \pm & 0.420 & 2.325 & \pm & 0.212 \\
\hline Liver & 1.946 & \pm & 0.408 & 1.433 & \pm & 0.605 & 1.545 & \pm & 0.635 & 1.089 & \pm & 0.066 \\
\hline Spleen & 0.578 & \pm & 0.156 & 0.419 & \pm & 0.048 & 0.489 & \pm & 0.096 & 0.485 & \pm & 0.029 \\
\hline Pancreas & 0.406 & \pm & 0.047 & 0.340 & \pm & 0.054 & 0.422 & \pm & 0.083 & 0.373 & \pm & 0.051 \\
\hline Stomach & 0.727 & \pm & 0.373 & 0.444 & \pm & 0.097 & 0.603 & \pm & 0.154 & 0.593 & \pm & 0.153 \\
\hline S. intestine & 13.049 & \pm & 3.020 & 12.437 & \pm & 6.386 & 4.931 & \pm & 2.811 & 0.776 & \pm & 0.196 \\
\hline L. intestine & 0.332 & \pm & 0.078 & 4.535 & \pm & 6.803 & 18.246 & \pm & 4.799 & 22.081 & \pm & 6.021 \\
\hline Kidneys & 17.053 & \pm & 2.372 & 10.443 & \pm & 0.757 & 9.250 & \pm & 1.538 & 7.759 & \pm & 0.658 \\
\hline Muscle & 0.351 & \pm & 0.046 & 0.296 & \pm & 0.117 & 0.327 & \pm & 0.088 & 0.325 & \pm & 0.073 \\
\hline Bone & 0.441 & \pm & 0.086 & 0.355 & \pm & 0.093 & 0.402 & \pm & 0.063 & 0.571 & \pm & 0.202 \\
\hline Skin & 1.439 & \pm & 0.207 & 1.217 & \pm & 0.184 & 1.482 & \pm & 0.329 & 1.377 & \pm & 0.148 \\
\hline Brain & 0.434 & \pm & 0.495 & 0.170 & \pm & 0.034 & 0.128 & \pm & 0.020 & 0.118 & \pm & 0.011 \\
\hline Tumor & 0.864 & \pm & 0.070 & 0.794 & \pm & 0.230 & 0.915 & \pm & 0.096 & 1.105 & \pm & 0.125 \\
\hline \multicolumn{13}{|c|}{ Tumor-to-tissue ratios (rel. u.) } \\
\hline Tumor/Blood & 0.29 & \pm & 0.07 & 0.35 & \pm & 0.12 & 0.36 & \pm & 0.10 & 0.48 & \pm & 0.08 \\
\hline Tumor/Liver & 0.44 & \pm & 0.10 & 0.6 & \pm & 0.3 & 0.6 & \pm & 0.3 & 1.01 & \pm & 0.13 \\
\hline Tumor/S. I. & 0.066 & \pm & 0.016 & 0.06 & \pm & 0.04 & 0.19 & \pm & 0.11 & 1.4 & \pm & 0.4 \\
\hline Tumor/L. I. & 2.60 & \pm & 0.64 & 0.17 & \pm & 0.27 & 0.050 & \pm & 0.014 & 0.050 & \pm & 0.015 \\
\hline Tumor/Kidney & 0.051 & \pm & 0.008 & 0.08 & \pm & 0.02 & 0.099 & \pm & 0.019 & 0.14 & \pm & 0.02 \\
\hline Tumor/Muscle & 2.5 & \pm & 0.4 & 2.7 & \pm & 1.3 & 2.8 & \pm & 0.8 & 3.4 & \pm & 0.9 \\
\hline Tumor/Bone & 2.0 & \pm & 0.4 & 2.2 & \pm & 0.9 & 2.3 & \pm & 0.4 & 1.9 & \pm & 0.7 \\
\hline
\end{tabular}

Table S6-2. Tissue uptake (mean $\% \mathrm{ID} / \mathrm{g} \pm \mathrm{SD}$ ) of ${ }^{18} \mathrm{~F}-\mathrm{AlF}-\mathrm{NOTA}-\mathrm{Var} 7$ administered via the lateral tail vein in female, BALB/c mice with orthotopic 4T1 breast cancer allografts implanted into the mammary fat pad. 


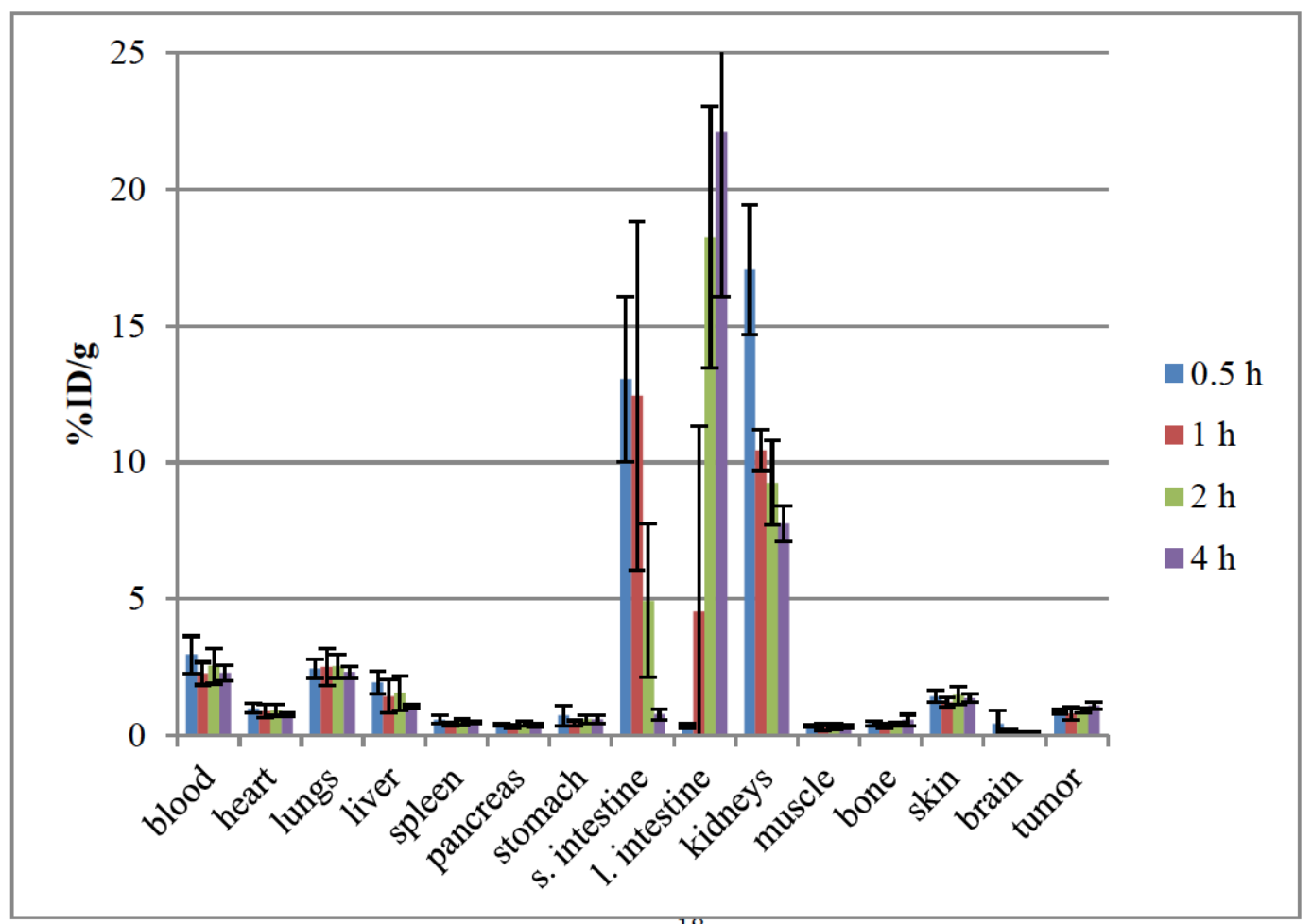

Fig. S6-1. Tissue uptake (mean $\% \mathrm{ID} / \mathrm{g} \pm \mathrm{SD})$ of ${ }^{18} \mathrm{~F}-\mathrm{AlF}-\mathrm{NOTA}-\mathrm{Var} 7(\mathrm{n}=5)$ administered via the lateral tail vein in female, BALB/c mice with orthotopic $4 \mathrm{~T} 1$ breast cancer allografts implanted into the mammary fat pad full graph from text. 


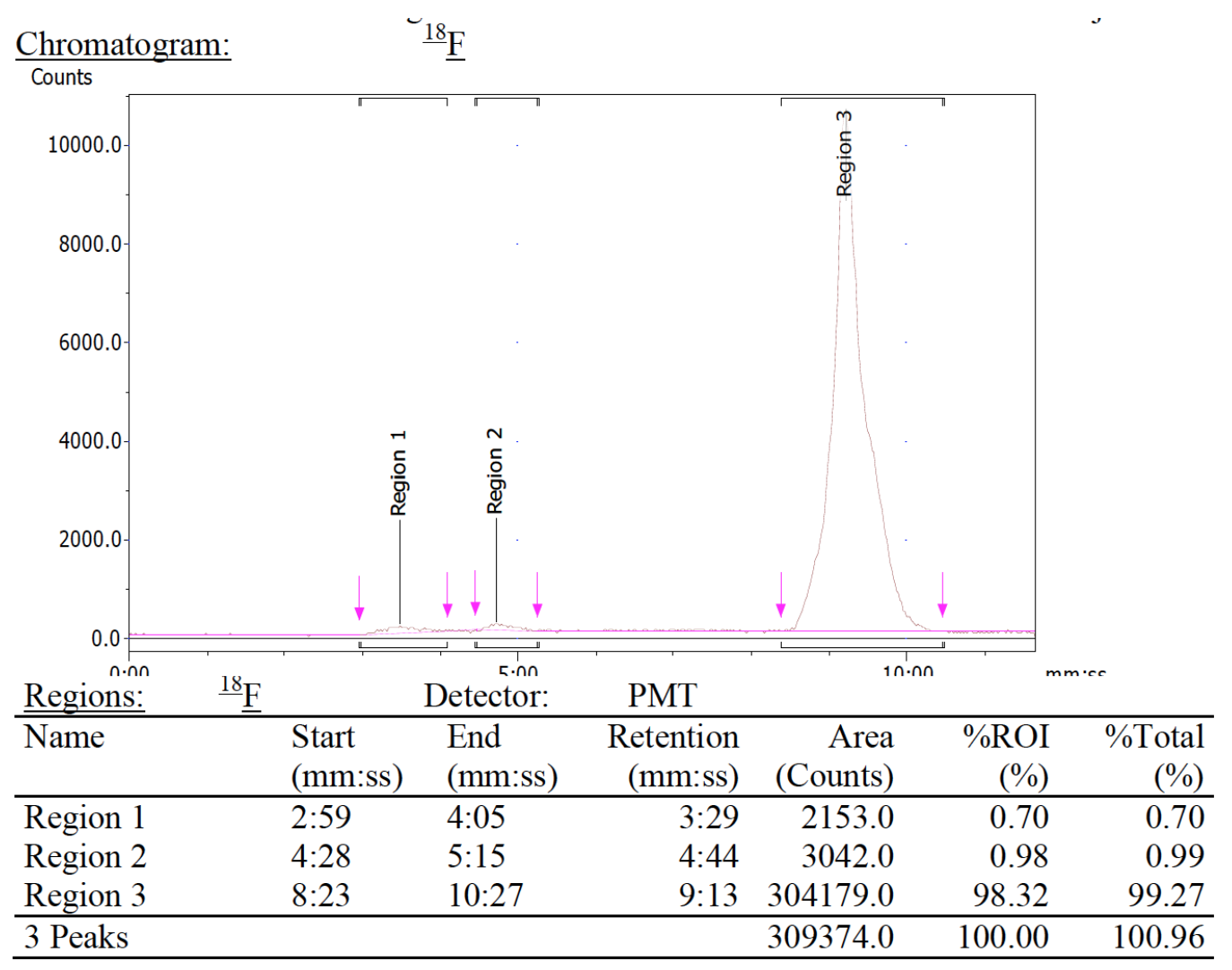

Fig. S6-2. HPLC Chromatogram of the Reconstituted ${ }^{18}$ F-AlF-NOTA-Var7 Injectate 


\section{${ }^{64} \mathrm{Cu}-\mathrm{NO2A-cysWT}$}

\begin{tabular}{|c|c|c|c|c|c|c|c|c|c|c|c|c|c|c|c|c|c|c|c|c|c|}
\hline \multirow{2}{*}{$\begin{array}{l}\text { Tissue } \\
\text { Blood }\end{array}$} & \multicolumn{3}{|c|}{$1 \mathrm{~h}(\mathrm{n}=4)$} & \multicolumn{3}{|c|}{$2 \mathrm{~h}(\mathrm{n}=4)$} & \multicolumn{3}{|c|}{$4 h(n=4)$} & \multicolumn{3}{|c|}{$4 h+$ glucose $(n=3)$} & \multicolumn{3}{|c|}{$12 \mathrm{~h}(\mathrm{n}=4)$} & \multicolumn{3}{|c|}{$24 \mathrm{~h}(\mathrm{n}=5)$} & \multicolumn{3}{|c|}{$48 \mathrm{~h}(\mathrm{n}=5)$} \\
\hline & 2.92 & \pm & 0.76 & 5.56 & \pm & 1.52 & 2.72 & \pm & 0.44 & 2.85 & \pm & 0.87 & 1.46 & \pm & 0.47 & 0.67 & \pm & 0.18 & 0.56 & \pm & 0.15 \\
\hline Heart & 0.70 & \pm & 0.12 & 0.73 & \pm & 0.17 & 0.51 & \pm & 0.04 & 0.45 & \pm & 0.03 & 0.45 & \pm & 0.07 & 0.47 & \pm & 0.04 & 0.48 & \pm & 0.07 \\
\hline Lungs & 5.27 & \pm & 0.71 & 3.06 & \pm & 0.52 & 2.88 & \pm & 0.46 & 2.57 & \pm & 0.09 & 1.79 & \pm & 0.34 & 1.72 & \pm & 0.10 & 1.08 & \pm & 0.19 \\
\hline Liver & 16.7 & \pm & 3.19 & 17.3 & \pm & 1.58 & 22.9 & \pm & 1.00 & 21.9 & \pm & 1.04 & 23.3 & \pm & 3.93 & 23.5 & \pm & 1.99 & 17.3 & \pm & 1.13 \\
\hline Spleen & 0.51 & \pm & 0.01 & 0.62 & \pm & 0.08 & 0.72 & \pm & 0.02 & 0.68 & \pm & 0.09 & 1.01 & \pm & 0.16 & 1.09 & \pm & 0.11 & 0.63 & \pm & 0.05 \\
\hline Pancreas & 0.26 & \pm & 0.05 & 0.44 & \pm & 0.18 & 0.40 & \pm & 0.04 & 0.28 & \pm & 0.00 & 0.40 & \pm & 0.05 & 0.46 & \pm & 0.06 & 0.41 & \pm & 0.04 \\
\hline Stomach & 0.65 & \pm & 0.07 & 0.69 & \pm & 0.04 & 1.06 & \pm & 0.02 & 0.93 & \pm & 0.27 & 1.33 & \pm & 0.24 & 1.65 & \pm & 0.28 & 1.16 & \pm & 0.10 \\
\hline S. intestine & 3.54 & \pm & 0.26 & 4.33 & \pm & 0.59 & 6.76 & \pm & 0.61 & 5.03 & \pm & 0.37 & 8.92 & \pm & 0.65 & 8.94 & \pm & 0.76 & 6.69 & \pm & 0.65 \\
\hline L. intestine & 0.78 & \pm & 0.12 & 1.73 & \pm & 0.13 & 3.19 & \pm & 0.29 & 2.49 & \pm & 0.17 & 6.17 & \pm & 0.50 & 6.26 & \pm & 0.78 & 5.28 & \pm & 0.90 \\
\hline Kidneys & 7.95 & \pm & 0.84 & 9.93 & \pm & 1.21 & 12.1 & \pm & 1.16 & 8.30 & \pm & 0.24 & 6.01 & \pm & 0.65 & 4.09 & \pm & 0.24 & 2.92 & \pm & 0.18 \\
\hline Muscle & 0.15 & \pm & 0.04 & 0.15 & \pm & 0.03 & 0.13 & \pm & 0.04 & 0.08 & \pm & 0.01 & 0.20 & \pm & 0.06 & 0.14 & \pm & 0.05 & 0.12 & \pm & 0.03 \\
\hline Bone & 0.14 & \pm & 0.07 & 0.10 & \pm & 0.02 & 0.12 & \pm & 0.02 & 0.12 & \pm & 0.02 & 0.13 & \pm & 0.01 & 0.14 & \pm & 0.03 & 0.10 & \pm & 0.01 \\
\hline Skin & 0.31 & \pm & 0.12 & 0.30 & \pm & 0.13 & 0.34 & \pm & 0.05 & 0.32 & \pm & 0.15 & 0.35 & \pm & 0.08 & 0.32 & \pm & 0.06 & 0.21 & \pm & 0.06 \\
\hline Brain & 0.30 & \pm & 0.03 & 0.22 & \pm & 0.05 & 0.25 & \pm & 0.04 & 0.16 & \pm & 0.05 & 0.13 & \pm & 0.02 & 0.19 & \pm & 0.00 & 0.20 & \pm & 0.01 \\
\hline Tumor & 0.36 & \pm & 0.10 & 0.48 & \pm & 0.15 & 0.80 & \pm & 0.13 & 0.49 & \pm & 0.10 & 1.12 & \pm & 0.28 & 1.29 & \pm & 0.45 & 0.82 & \pm & 0.22 \\
\hline
\end{tabular}

Table S7-1. Tissue uptake (mean $\%$ ID \pm SD) of ${ }^{64} \mathrm{Cu}-\mathrm{NO} 2 \mathrm{~A}$-cysWT administered via

the lateral tail vein in female, BALB/c mice with orthotopic 4T1 breast cancer allografts implanted into the mammary fat pad. 


\begin{tabular}{|c|c|c|c|c|c|c|c|c|c|c|c|c|c|c|c|c|c|c|c|c|c|}
\hline \multirow{2}{*}{$\begin{array}{l}\text { Tissue } \\
\text { Blood }\end{array}$} & \multicolumn{3}{|c|}{$1 \mathrm{~h}(\mathrm{n}=4)$} & \multicolumn{3}{|c|}{$2 h(n=4)$} & \multicolumn{3}{|c|}{$4 \mathrm{~h}(\mathrm{n}=4)$} & \multicolumn{3}{|c|}{$4 \mathrm{~h}+$ glucose $(n=3)$} & \multicolumn{3}{|c|}{$12 \mathrm{~h}(\mathrm{n}=4)$} & \multicolumn{3}{|c|}{24 h $(n=5)$} & \multicolumn{3}{|c|}{$48 \mathrm{~h}(\mathrm{n}=5)$} \\
\hline & 26.5 & \pm & 1.12 & 24.6 & \pm & 2.61 & 17.6 & \pm & 0.86 & 13.8 & \pm & 0.82 & 6.40 & \pm & 0.65 & 3.64 & \pm & 0.15 & 1.90 & \pm & 0.11 \\
\hline Heart & 6.87 & \pm & 1.05 & 7.08 & \pm & 1.66 & 5.52 & \pm & 0.15 & 4.11 & \pm & 0.35 & 4.01 & \pm & 0.67 & 4.36 & \pm & 0.21 & 5.01 & \pm & 0.49 \\
\hline Lungs & 19.2 & \pm & 2.29 & 15.4 & \pm & 1.78 & 12.3 & \pm & 1.55 & 10.1 & \pm & 1.23 & 8.18 & \pm & 1.63 & 6.73 & \pm & 0.40 & 6.38 & \pm & 0.25 \\
\hline Liver & 17.5 & \pm & 1.14 & 20.8 & \pm & 2.62 & 21.9 & \pm & 0.64 & 19.5 & \pm & 2.13 & 19.2 & \pm & 3.33 & 23.3 & \pm & 1.79 & 17.9 & \pm & 1.74 \\
\hline Spleen & 4.64 & \pm & 0.23 & 5.65 & \pm & 0.80 & 7.07 & \pm & 0.44 & 6.50 & \pm & 0.90 & 8.79 & \pm & 1.60 & 9.80 & \pm & 0.56 & 6.07 & \pm & 0.72 \\
\hline Pancreas & 2.27 & \pm & 0.14 & 2.83 & \pm & 0.51 & 3.00 & \pm & 0.08 & 2.15 & \pm & 0.25 & 2.71 & \pm & 0.38 & 3.34 & \pm & 0.40 & 2.87 & \pm & 0.08 \\
\hline Stomach & 1.77 & \pm & 0.19 & 1.83 & \pm & 0.24 & 1.82 & \pm & 0.13 & 2.32 & \pm & 0.15 & 2.81 & \pm & 0.45 & 4.30 & \pm & 0.90 & 3.93 & \pm & 0.89 \\
\hline S. intestine & 2.74 & \pm & 0.15 & 3.53 & \pm & 0.44 & 4.56 & \pm & 0.26 & 3.43 & \pm & 0.33 & 6.47 & \pm & 0.83 & 6.33 & \pm & 0.45 & 5.36 & \pm & 0.65 \\
\hline L. intestine & 1.03 & \pm & 0.08 & 2.16 & \pm & 0.27 & 4.09 & \pm & 0.34 & 3.07 & \pm & 0.13 & 6.52 & \pm & 0.86 & 7.21 & \pm & 1.05 & 5.97 & \pm & 0.98 \\
\hline Kidneys & 27.3 & \pm & 2.15 & 34.3 & \pm & 3.96 & 40.6 & \pm & 4.04 & 26.3 & \pm & 0.92 & 18.2 & \pm & 3.39 & 13.3 & \pm & 0.58 & 9.86 & \pm & 0.77 \\
\hline Muscle & 1.04 & \pm & 0.12 & 1.37 & \pm & 0.46 & 1.15 & \pm & 0.08 & 0.82 & \pm & 0.07 & 1.25 & \pm & 0.13 & 1.06 & \pm & 0.11 & 0.94 & \pm & 0.12 \\
\hline Bone & 2.31 & \pm & 0.19 & 2.58 & \pm & 0.29 & 3.04 & \pm & 0.11 & 2.13 & \pm & 0.38 & 3.04 & \pm & 0.28 & 3.11 & \pm & 0.36 & 1.99 & \pm & 0.15 \\
\hline Skin & 2.53 & \pm & 0.53 & 2.92 & \pm & 0.59 & 3.23 & \pm & 0.12 & 2.36 & \pm & 0.79 & 3.30 & \pm & 0.51 & 3.22 & \pm & 0.15 & 2.14 & \pm & 0.13 \\
\hline Brain & 0.75 & \pm & 0.05 & 0.58 & \pm & 0.12 & 0.62 & \pm & 0.06 & 0.39 & \pm & 0.09 & 0.38 & \pm & 0.06 & 0.48 & \pm & 0.03 & 0.51 & \pm & 0.02 \\
\hline Tumor & 5.03 & \pm & 1.48 & 7.55 & \pm & 2.15 & 11.7 & \pm & 1.71 & 7.10 & \pm & 0.81 & 14.3 & \pm & 2.72 & 12.8 & \pm & 1.55 & 6.16 & \pm & 0.48 \\
\hline \multicolumn{22}{|c|}{ Tumor-to-tissue ratios (rel. u.) } \\
\hline Tumor/Blood & 0.19 & \pm & 0.06 & 0.31 & \pm & 0.09 & 0.66 & \pm & 0.10 & 0.52 & \pm & 0.07 & 2.2 & \pm & 0.5 & 3.5 & \pm & 0.5 & 3.2 & \pm & 0.3 \\
\hline Tumor/Liver & 0.29 & \pm & 0.09 & 0.36 & \pm & 0.11 & 0.53 & \pm & 0.08 & 0.36 & \pm & 0.06 & 0.75 & \pm & 0.19 & 0.55 & \pm & 0.08 & 0.34 & \pm & 0.04 \\
\hline Tumor/S. I. & 1.8 & \pm & 0.5 & 2.1 & \pm & 0.7 & 2.6 & \pm & 0.4 & 2.1 & \pm & 0.3 & 2.2 & \pm & 0.5 & 2.0 & \pm & 0.3 & 1.15 & \pm & 0.17 \\
\hline Tumor/L. I. & 4.9 & \pm & 1.5 & 3.5 & \pm & 1.1 & 2.9 & \pm & 0.5 & 2.3 & \pm & 0.3 & 2.2 & \pm & 0.5 & 1.8 & \pm & 0.3 & 1.03 & \pm & 0.19 \\
\hline Tumor/Kidney & 0.18 & \pm & 0.06 & 0.22 & \pm & 0.07 & 0.29 & \pm & 0.05 & 0.27 & \pm & 0.03 & 0.8 & \pm & 0.2 & 0.96 & \pm & 0.12 & 0.62 & \pm & 0.07 \\
\hline Tumor/Muscle & 4.8 & \pm & 1.5 & 5.5 & \pm & 2.4 & 10.1 & \pm & 1.7 & 8.6 & \pm & 1.2 & 11 & \pm & 2 & 12 & \pm & 2 & 6.6 & \pm & 1.0 \\
\hline Tumor/Bone & 2.2 & \pm & 0.7 & 2.9 & \pm & 0.9 & 3.8 & \pm & 0.6 & 3.3 & \pm & 0.7 & 4.7 & \pm & 1.0 & 4.1 & \pm & 0.7 & 3.1 & \pm & 0.3 \\
\hline
\end{tabular}

Table S7-2. Tissue uptake (mean $\% \mathrm{ID} / \mathrm{g} \pm \mathrm{SD}$ ) of ${ }^{64} \mathrm{Cu}-\mathrm{NO} 2 \mathrm{~A}-\mathrm{cys} \mathrm{WT}$ administered via the lateral tail vein in female, BALB/c mice with orthotopic $4 \mathrm{~T} 1$ breast cancer allografts implanted into the mammary fat pad. 


\section{${ }^{64} \mathrm{C}$ u-N O 2 A - cys W T}

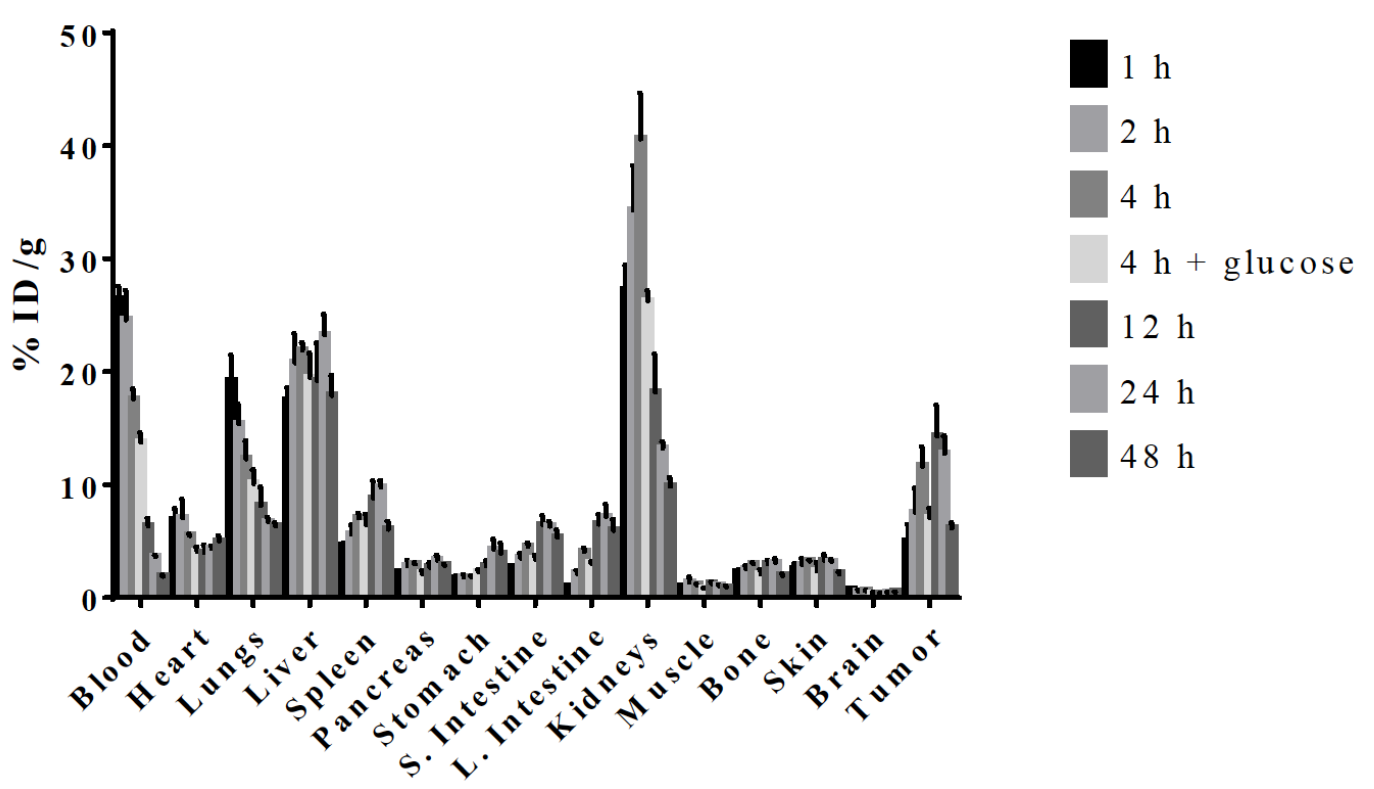

Fig. S7-1. Tissue uptake (mean $\% \mathrm{ID} / \mathrm{g} \pm \mathrm{SD})$ of ${ }^{64} \mathrm{Cu}-\mathrm{NO} 2 \mathrm{~A}-\mathrm{cysWT}(\mathrm{n}=3-5)$ administered via the lateral tail vein in female, BALB/c mice with orthotopic 4T1 breast cancer allografts implanted into the mammary fat pad full graph from text. Glucose was administered i.p. 30 min prior to tracer injection. 


\section{BIOSCAN}

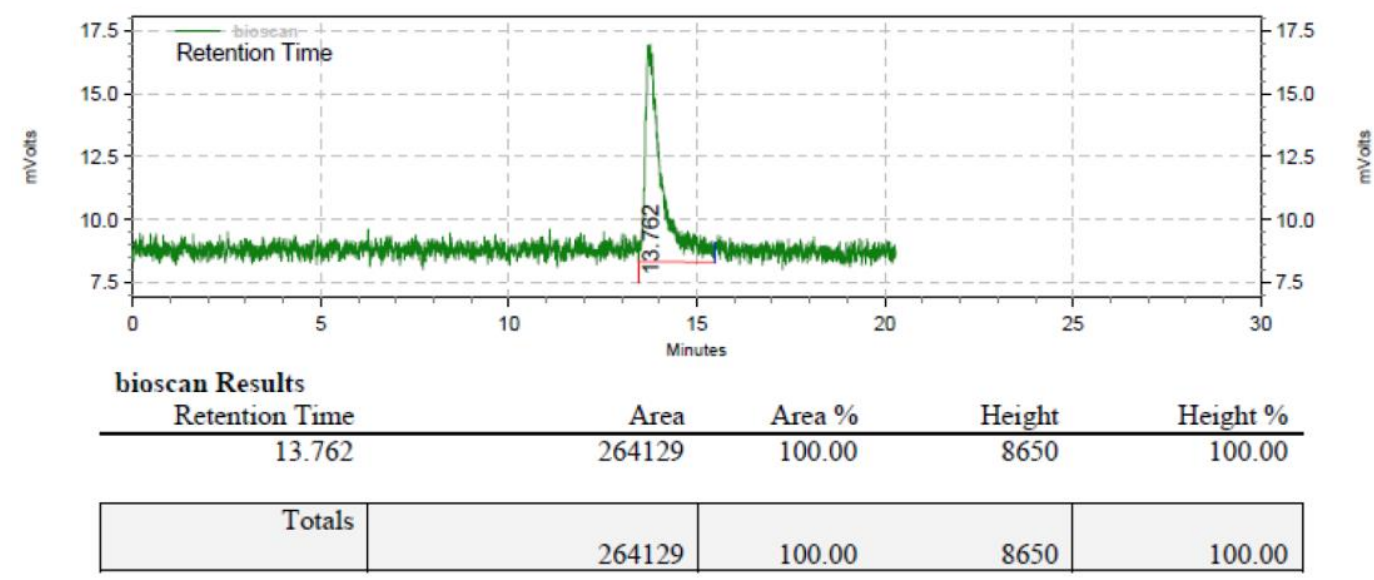

Fig. S7-2. HPLC Chromatogram of the Reconstituted ${ }^{64} \mathrm{Cu}-\mathrm{NO} 2 \mathrm{~A}-\mathrm{cys}$ WT Injectate 


\section{${ }^{64} \mathrm{Cu}-\mathrm{NO2A-cysVar7}$}

\begin{tabular}{|c|c|c|c|c|c|c|c|c|c|c|c|c|c|c|c|c|c|c|c|c|c|}
\hline \multirow{2}{*}{$\begin{array}{l}\text { Tissue } \\
\text { Blood }\end{array}$} & \multicolumn{3}{|c|}{$1 \mathrm{~h}(\mathrm{n}=4)$} & \multicolumn{3}{|c|}{$2 \mathrm{~h}(\mathrm{n}=4)$} & \multicolumn{3}{|c|}{$4 h(n=4)$} & \multicolumn{3}{|c|}{$4 \mathrm{~h}+$ glucose $(n=4)$} & \multicolumn{3}{|c|}{$12 h(n=4)$} & \multicolumn{3}{|c|}{$24 \mathrm{~h}(\mathrm{n}=5)$} & \multicolumn{3}{|c|}{$48 h(n=5)$} \\
\hline & 1.96 & \pm & 1.25 & 0.87 & \pm & 0.41 & 0.45 & \pm & 0.09 & 0.39 & \pm & 0.15 & 0.74 & \pm & 0.38 & 0.47 & \pm & 0.10 & 0.28 & \pm & 0.12 \\
\hline Heart & 0.24 & \pm & 0.04 & 0.21 & \pm & 0.01 & 0.28 & \pm & 0.03 & 0.25 & \pm & 0.02 & 0.49 & \pm & 0.11 & 0.46 & \pm & 0.08 & 0.36 & \pm & 0.04 \\
\hline Lungs & 1.52 & \pm & 0.27 & 1.02 & \pm & 0.19 & 1.04 & \pm & 0.06 & 0.86 & \pm & 0.12 & 1.56 & \pm & 0.13 & 1.36 & \pm & 0.16 & 0.79 & \pm & 0.07 \\
\hline Liver & 6.14 & \pm & 0.70 & 9.31 & \pm & 0.68 & 15.7 & \pm & 1.3 & 13.7 & \pm & 0.9 & 18.8 & \pm & 2.8 & 16.4 & \pm & 1.7 & 10.3 & \pm & 1.1 \\
\hline Spleen & 0.22 & \pm & 0.02 & 0.26 & \pm & 0.02 & 0.35 & \pm & 0.04 & 0.35 & \pm & 0.02 & 0.68 & \pm & 0.20 & 0.55 & \pm & 0.11 & 0.23 & \pm & 0.02 \\
\hline Pancreas & 0.14 & \pm & 0.01 & 0.18 & \pm & 0.02 & 0.28 & \pm & 0.05 & 0.24 & \pm & 0.03 & 0.38 & \pm & 0.05 & 0.40 & \pm & 0.04 & 0.24 & \pm & 0.07 \\
\hline Stomach & 0.44 & \pm & 0.10 & 0.65 & \pm & 0.04 & 1.48 & \pm & 0.03 & 1.10 & \pm & 0.36 & 1.83 & \pm & 0.30 & 1.33 & \pm & 0.16 & 1.02 & \pm & 0.20 \\
\hline S. intestine & 2.66 & \pm & 0.29 & 4.74 & \pm & 0.92 & 9.04 & \pm & 0.95 & 5.91 & \pm & 0.44 & 9.73 & \pm & 0.76 & 8.21 & \pm & 0.34 & 4.70 & \pm & 0.27 \\
\hline L. intestine & 0.85 & \pm & 0.17 & 2.07 & \pm & 0.26 & 6.39 & \pm & 0.68 & 4.30 & \pm & 0.87 & 8.64 & \pm & 1.32 & 5.79 & \pm & 0.27 & 4.52 & \pm & 0.51 \\
\hline Kidneys & 35.5 & \pm & 3.92 & 40.5 & \pm & 1.57 & 29.7 & \pm & 3.4 & 25.1 & \pm & 2.0 & 8.14 & \pm & 0.52 & 5.23 & \pm & 0.47 & 2.97 & \pm & 0.19 \\
\hline Muscle & 0.10 & \pm & 0.02 & 0.08 & \pm & 0.01 & 0.09 & \pm & 0.02 & 0.09 & \pm & 0.03 & 0.10 & \pm & 0.02 & 0.11 & \pm & 0.03 & 0.08 & \pm & 0.01 \\
\hline Bone & 0.03 & \pm & 0.01 & 0.03 & \pm & 0.02 & 0.14 & \pm & 0.05 & 0.07 & \pm & 0.00 & 0.10 & \pm & 0.02 & 0.08 & \pm & 0.01 & 0.05 & \pm & 0.01 \\
\hline Skin & 0.39 & \pm & 0.04 & 0.32 & \pm & 0.10 & 0.29 & \pm & 0.02 & 0.31 & \pm & 0.04 & 0.33 & \pm & 0.03 & 0.24 & \pm & 0.05 & 0.14 & \pm & 0.01 \\
\hline Brain & 0.08 & \pm & 0.02 & 0.06 & \pm & 0.01 & 0.10 & \pm & 0.01 & 0.08 & \pm & 0.01 & 0.18 & \pm & 0.04 & 0.25 & \pm & 0.02 & 0.21 & \pm & 0.03 \\
\hline Tumor & 0.38 & \pm & 0.10 & 0.48 & \pm & 0.09 & 0.71 & \pm & 0.13 & 0.63 & \pm & 0.11 & 0.78 & \pm & 0.24 & 0.57 & \pm & 0.24 & 0.37 & \pm & 0.03 \\
\hline
\end{tabular}

Table S8-1. Tissue uptake (mean \%ID $\pm \mathrm{SD}$ ) of ${ }^{64} \mathrm{Cu}-\mathrm{NO} 2 \mathrm{~A}$-cysVar7 administered via the lateral tail vein in female, BALB/c mice with orthotopic $4 \mathrm{~T} 1$ breast cancer allografts implanted into the mammary fat pad. 


\begin{tabular}{|c|c|c|c|c|c|c|c|c|c|c|c|c|c|c|c|c|c|c|c|c|c|}
\hline \multirow{2}{*}{$\begin{array}{l}\text { Tissue } \\
\text { Blood }\end{array}$} & \multicolumn{3}{|c|}{$1 \mathrm{~h}(\mathrm{n}=4)$} & \multicolumn{3}{|c|}{$2 \mathrm{~h}(\mathrm{n}=4)$} & \multicolumn{3}{|c|}{$4 h(n=4)$} & \multicolumn{3}{|c|}{$4 h+$ glucose $(n=4)$} & \multicolumn{3}{|c|}{$12 h(n=4)$} & \multicolumn{3}{|c|}{$24 h(n=5)$} & \multicolumn{3}{|c|}{$48 h(n=5)$} \\
\hline & 7.21 & \pm & 0.72 & 3.78 & \pm & 1.22 & 2.31 & \pm & 0.18 & 2.36 & \pm & 0.23 & 2.62 & \pm & 0.61 & 2.46 & \pm & 0.35 & 1.43 & \pm & 0.07 \\
\hline Heart & 2.65 & \pm & 0.33 & 2.14 & \pm & 0.28 & 2.68 & \pm & 0.33 & 2.46 & \pm & 0.25 & 4.41 & \pm & 0.88 & 4.46 & \pm & 0.60 & 4.01 & \pm & 0.18 \\
\hline Lungs & 6.26 & \pm & 0.72 & 4.01 & \pm & 0.46 & 4.21 & \pm & 0.23 & 3.85 & \pm & 0.23 & 5.79 & \pm & 0.57 & 6.04 & \pm & 0.81 & 5.08 & \pm & 0.30 \\
\hline Liver & 7.52 & \pm & 0.37 & 10.2 & \pm & 0.70 & 15.6 & \pm & 1.58 & 16.7 & \pm & 2.00 & 17.1 & \pm & 2.93 & 18.4 & \pm & 2.35 & 11.9 & \pm & 0.68 \\
\hline Spleen & 2.00 & \pm & 0.12 & 2.29 & \pm & 0.25 & 3.31 & \pm & 0.24 & 3.47 & \pm & 0.28 & 5.59 & \pm & 0.88 & 5.43 & \pm & 0.72 & 2.54 & \pm & 0.18 \\
\hline Pancreas & 1.12 & \pm & 0.08 & 1.23 & \pm & 0.14 & 1.84 & \pm & 0.10 & 1.98 & \pm & 0.14 & 2.52 & \pm & 0.24 & 2.83 & \pm & 0.29 & 2.33 & \pm & 0.26 \\
\hline Stomach & 1.74 & \pm & 0.62 & 2.02 & \pm & 0.42 & 3.33 & \pm & 0.31 & 3.27 & \pm & 0.83 & 4.09 & \pm & 1.39 & 4.68 & \pm & 0.76 & 2.67 & \pm & 0.81 \\
\hline S. intestine & 2.32 & \pm & 0.30 & 4.02 & \pm & 0.55 & 6.37 & \pm & 0.11 & 4.93 & \pm & 0.57 & 6.96 & \pm & 0.73 & 6.52 & \pm & 0.56 & 4.08 & \pm & 0.29 \\
\hline L. intestine & 1.24 & \pm & 0.21 & 2.82 & \pm & 0.21 & 8.19 & \pm & 0.85 & 6.63 & \pm & 1.37 & 9.88 & \pm & 1.28 & 7.42 & \pm & 0.89 & 6.20 & \pm & 0.96 \\
\hline Kidneys & 145.5 & \pm & 12.80 & 139.0 & \pm & 13.4 & 95.5 & \pm & 11.9 & 93.8 & \pm & 5.16 & 27.6 & \pm & 4.46 & 19.2 & \pm & 3.08 & 9.73 & \pm & 0.33 \\
\hline Muscle & 0.98 & \pm & 0.29 & 0.60 & \pm & 0.06 & 0.64 & \pm & 0.07 & 0.73 & \pm & 0.08 & 0.82 & \pm & 0.16 & 0.90 & \pm & 0.15 & 0.71 & \pm & 0.05 \\
\hline Bone & 0.93 & \pm & 0.19 & 0.95 & \pm & 0.24 & 1.57 & \pm & 0.18 & 1.40 & \pm & 0.14 & 2.12 & \pm & 0.26 & 1.91 & \pm & 0.22 & 1.11 & \pm & 0.05 \\
\hline Skin & 2.53 & \pm & 0.28 & 2.12 & \pm & 0.11 & 2.59 & \pm & 0.05 & 2.63 & \pm & 0.13 & 2.79 & \pm & 0.46 & 2.47 & \pm & 0.20 & 1.51 & \pm & 0.15 \\
\hline Brain & 0.20 & \pm & 0.03 & 0.16 & \pm & 0.02 & 0.25 & \pm & 0.02 & 0.22 & \pm & 0.03 & 0.48 & \pm & 0.06 & 0.62 & \pm & 0.07 & 0.53 & \pm & 0.04 \\
\hline Tumor & 6.54 & \pm & 0.35 & 7.02 & \pm & 0.82 & 9.07 & \pm & 1.81 & 11.2 & \pm & 0.55 & 9.60 & \pm & 0.90 & 7.28 & \pm & 1.21 & 3.90 & \pm & 0.58 \\
\hline \multicolumn{22}{|c|}{ Tumor-to-tissue ratios (rel. u.) } \\
\hline Tumor/Blood & 0.91 & \pm & 0.10 & 1.9 & \pm & 0.6 & 3.9 & \pm & 0.8 & 4.7 & \pm & 0.5 & 3.7 & \pm & 0.9 & 3.0 & \pm & 0.6 & 2.7 & \pm & 0.4 \\
\hline Tumor/Liver & 0.87 & \pm & 0.06 & 0.69 & \pm & 0.09 & 0.58 & \pm & 0.13 & 0.67 & \pm & 0.09 & 0.56 & \pm & 0.11 & 0.40 & \pm & 0.08 & 0.33 & \pm & 0.05 \\
\hline Tumor/S. I. & 2.8 & \pm & 0.4 & 1.7 & \pm & 0.3 & 1.4 & \pm & 0.3 & 2.3 & \pm & 0.3 & 1.4 & \pm & 0.2 & 1.1 & \pm & 0.2 & 0.96 & \pm & 0.16 \\
\hline Tumor/L. I. & 5.3 & \pm & 0.9 & 2.5 & \pm & 0.3 & 1.1 & \pm & 0.2 & 1.7 & \pm & 0.4 & 1.0 & \pm & 0.2 & 1.0 & \pm & 0.2 & 0.63 & \pm & 0.14 \\
\hline Tumor/Kidney & 0.045 & \pm & 0.005 & 0.050 & \pm & 0.008 & 0.09 & \pm & 0.02 & 0.119 & \pm & 0.009 & 0.35 & \pm & 0.06 & 0.38 & \pm & 0.09 & 0.40 & \pm & 0.06 \\
\hline Tumor/Muscle & 7 & \pm & 2 & 11.7 & \pm & 1.8 & 14 & \pm & & 15.4 & \pm & 1.9 & 12 & \pm & 2 & 8 & \pm & 2 & 5.5 & \pm & 0.9 \\
\hline Tumor/Bone & 7.1 & \pm & 1.5 & 7 & \pm & 2 & 5.8 & \pm & 1.3 & 7.9 & \pm & 0.9 & 4.5 & \pm & 0.7 & 3.8 & \pm & 0.8 & 3.5 & \pm & 0.5 \\
\hline
\end{tabular}

Table S8-2. Tissue uptake (mean $\% \mathrm{ID} / \mathrm{g} \pm \mathrm{SD}$ ) of ${ }^{64} \mathrm{Cu}-\mathrm{NO} 2 \mathrm{~A}-\mathrm{cys}$ Var7 administered via the lateral tail vein in female, $\mathrm{BALB} / \mathrm{c}$ mice with orthotopic $4 \mathrm{~T} 1$ breast cancer allografts implanted into the mammary fat pad. 


$$
{ }^{64} \mathrm{Cu}-\mathrm{N} O 2 \mathrm{~A}-\mathrm{cys} \mathrm{V} \text { a r } 7
$$

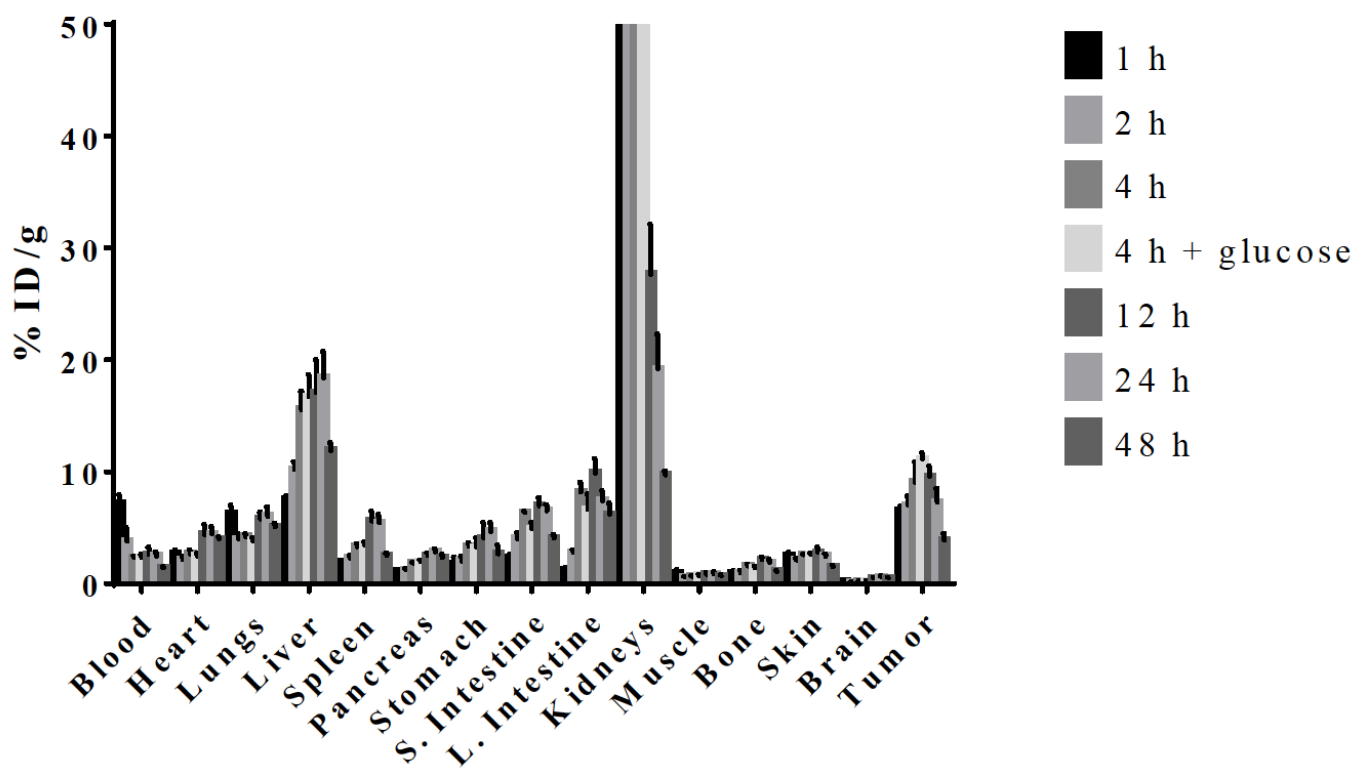

Fig. S8-1. Tissue uptake (mean \%ID/g \pm SD) of ${ }^{64} \mathrm{Cu}-\mathrm{NO} 2 \mathrm{~A}-\mathrm{cys} V a r 7(\mathrm{n}=4-5)$ administered via the lateral tail vein in female, BALB/c mice with orthotopic 4T1 breast cancer allografts implanted into the mammary fat pad full graph from text. Glucose was administered i.p. 30 min prior to tracer injection. 


\section{BIOSCAN}

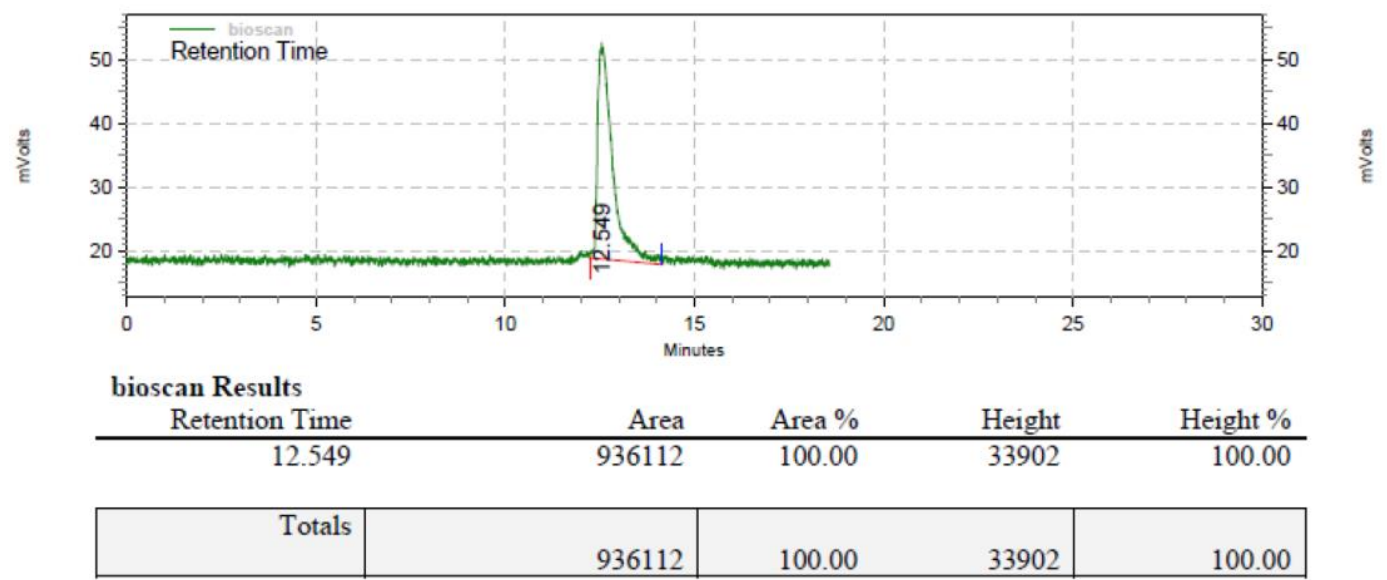

Fig. S8-2. HPLC Chromatogram of the Reconstituted ${ }^{64} \mathrm{Cu}-\mathrm{NO} 2 \mathrm{~A}-\mathrm{cys}$ Var7 Injectate 


\section{${ }^{18}$ F-AIF-NO2A-cysWT}

\begin{tabular}{|c|c|c|c|c|c|c|c|c|c|c|c|c|c|c|c|c|c|}
\hline \multirow{2}{*}{$\begin{array}{l}\text { Tissue } \\
\text { Blood }\end{array}$} & \multicolumn{3}{|c|}{$0.5 \mathrm{~h}(\mathrm{n}=4)$} & \multicolumn{3}{|c|}{$1 h(n=4)$} & \multicolumn{3}{|c|}{$2 h(n=4)$} & \multicolumn{3}{|c|}{$4 h(n=4)$} & \multicolumn{2}{|c|}{$4 h+$ glucose $(n=4)$} & \multicolumn{3}{|c|}{$6 h(n=4)$} \\
\hline & 12.3 & \pm & 5.05 & 5.71 & \pm & 0.45 & 6.72 & & 3.43 & 6.20 & \pm & 3.06 & 4.16 & \pm 1.89 & 2.39 & \pm & 0.99 \\
\hline Heart & 0.59 & \pm & 0.07 & 0.66 & \pm & 0.25 & 0.51 & \pm & 0.08 & 0.48 & \pm & 0.15 & 0.35 & \pm 0.05 & 0.66 & \pm & 0.18 \\
\hline Lungs & 3.72 & \pm & 2.15 & 4.09 & \pm & 0.32 & 2.84 & \pm & 0.29 & 1.74 & \pm & 0.23 & 1.99 & \pm 0.13 & 0.98 & \pm & 0.21 \\
\hline Liver & 13.2 & \pm & 0.74 & 12.9 & \pm & 0.61 & 12.2 & \pm & 0.79 & 14.4 & \pm & 1.53 & 17.9 & \pm 1.03 & 13.1 & \pm & 1.32 \\
\hline Spleen & 0.43 & \pm & 0.04 & 0.40 & \pm & 0.08 & 0.43 & \pm & 0.02 & 0.60 & \pm & 0.03 & 0.63 & \pm 0.08 & 0.93 & \pm & 0.26 \\
\hline Pancreas & 0.29 & \pm & 0.04 & 0.33 & \pm & 0.08 & 0.27 & \pm & 0.04 & 0.27 & \pm & 0.07 & 0.26 & \pm 0.08 & 0.57 & \pm & 0.25 \\
\hline Stomach & 0.50 & \pm & 0.04 & 0.42 & \pm & 0.05 & 0.50 & \pm & 0.12 & 0.38 & \pm & 0.01 & 0.60 & \pm 0.15 & 0.83 & \pm & 0.23 \\
\hline S. intestine & 2.62 & \pm & 0.29 & 2.55 & \pm & 0.38 & 3.14 & \pm & 0.53 & 3.16 & \pm & 0.18 & 2.82 & \pm 0.35 & 3.58 & \pm & 0.30 \\
\hline L. intestine & 0.60 & \pm & 0.21 & 0.68 & \pm & 0.24 & 0.78 & \pm & 0.03 & 1.17 & \pm & 0.32 & 1.09 & \pm 0.26 & 1.64 & \pm & 0.28 \\
\hline Kidneys & 4.95 & \pm & 0.53 & 6.85 & \pm & 0.74 & 9.95 & \pm & 1.17 & 15.0 & \pm & 0.72 & 11.4 & \pm 1.58 & 17.7 & \pm & 2.34 \\
\hline Muscle & 0.12 & \pm & 0.02 & 0.12 & \pm & 0.01 & 0.14 & \pm & 0.02 & 0.15 & \pm & 0.03 & 0.10 & \pm 0.02 & 0.45 & \pm & 0.31 \\
\hline Bone & 0.07 & \pm & 0.02 & 0.05 & \pm & 0.01 & 0.05 & \pm & 0.02 & 0.05 & \pm & 0.02 & 0.16 & \pm 0.07 & 0.47 & \pm & 0.26 \\
\hline Skin & 0.28 & \pm & 0.08 & 0.35 & \pm & 0.06 & 0.51 & \pm & 0.07 & 0.50 & \pm & 0.08 & 0.20 & \pm 0.09 & 0.64 & \pm & 0.24 \\
\hline Brain & 0.26 & \pm & 0.04 & 0.18 & \pm & 0.03 & 0.14 & \pm & 0.02 & 0.13 & \pm & 0.03 & 0.13 & \pm 0.02 & 0.47 & \pm & 0.24 \\
\hline Tumor & 0.22 & \pm & 0.06 & 0.25 & \pm & 0.08 & 0.35 & \pm & 0.10 & 0.55 & \pm & 0.12 & 0.48 & \pm 0.15 & 0.89 & \pm & 0.32 \\
\hline
\end{tabular}

Table S9-1. Tissue uptake (mean $\%$ ID \pm SD) of ${ }^{18} \mathrm{~F}$-AlF-NO2A-cysWT administered via the lateral tail vein in female, BALB/c mice with orthotopic 4T1 breast cancer allografts implanted into the mammary fat pad. 


\begin{tabular}{|c|c|c|c|c|c|c|c|c|c|c|c|c|c|c|c|c|c|}
\hline \multirow{2}{*}{$\begin{array}{l}\text { Tissue } \\
\text { Blood }\end{array}$} & \multicolumn{2}{|c|}{$0.5 \mathrm{~h}(\mathrm{n}=4)$} & \multicolumn{3}{|c|}{$1 \mathrm{~h}(\mathrm{n}=4)$} & \multicolumn{3}{|c|}{$2 h(n=4)$} & \multicolumn{3}{|c|}{$4 h(n=4)$} & \multicolumn{3}{|c|}{$4 h+$ glucose $(n=4)$} & \multicolumn{3}{|c|}{$6 h(n=4)$} \\
\hline & 35.8 & \pm 3.22 & 24.3 & \pm & 1.66 & 21.6 & \pm & 1.75 & 15.9 & \pm & 0.63 & 14.0 & \pm & 0.73 & 10.0 & \pm & 1.91 \\
\hline Heart & 6.33 & \pm 0.75 & 6.18 & \pm & 1.69 & 5.40 & \pm & 0.59 & 4.78 & \pm & 0.53 & 3.79 & \pm & 0.48 & 7.48 & \pm & 2.47 \\
\hline Lungs & 17.0 & \pm 5.66 & 15.1 & \pm & 1.58 & 12.4 & \pm & 0.44 & 8.73 & \pm & 0.84 & 7.27 & \pm & 0.26 & 6.34 & \pm & 1.53 \\
\hline Liver & 16.3 & \pm 1.39 & 13.7 & \pm & 1.52 & 15.4 & \pm & 1.23 & 12.6 & \pm & 11.4 & 18.4 & \pm & 1.31 & 14.5 & \pm & 1.60 \\
\hline Spleen & 4.06 & \pm 0.43 & 3.64 & \pm & 0.31 & 4.12 & \pm & 0.15 & 5.31 & \pm & 0.34 & 5.89 & \pm & 0.45 & 8.25 & \pm & 2.44 \\
\hline Pancreas & 2.09 & \pm 0.09 & 1.97 & \pm & 0.29 & 1.86 & \pm & 0.18 & 2.10 & \pm & 0.43 & 2.01 & \pm & 0.58 & 5.00 & \pm & 2.53 \\
\hline Stomach & 2.47 & \pm 0.70 & 1.35 & \pm & 0.15 & 2.01 & \pm & 0.34 & 1.31 & \pm & 0.96 & 1.96 & \pm & 0.16 & 2.05 & \pm & 1.11 \\
\hline S. intestine & 2.58 & \pm 0.39 & 2.12 & \pm & 0.19 & 2.83 & \pm & 0.27 & 2.85 & \pm & 0.31 & 2.34 & \pm & 0.20 & 3.15 & \pm & 0.54 \\
\hline L. intestine & 0.93 & \pm 0.21 & 0.92 & \pm & 0.21 & 1.19 & \pm & 0.08 & 1.47 & \pm & 0.14 & 1.61 & \pm & 0.26 & 2.54 & \pm & 0.34 \\
\hline Kidneys & 18.8 & \pm 1.96 & 23.4 & \pm & 2.15 & 36.1 & \pm & 6.63 & 59.1 & \pm & 3.63 & 42.6 & \pm & 10.9 & 64.8 & \pm & 14.3 \\
\hline Muscle & 0.96 & \pm 0.10 & 0.93 & \pm & 0.05 & 1.18 & \pm & 0.04 & 1.23 & \pm & 0.17 & 0.81 & \pm & 0.12 & 3.71 & \pm & 2.52 \\
\hline Bone & 2.43 & \pm 0.39 & 1.82 & \pm & 0.19 & 1.99 & \pm & 0.30 & 1.94 & \pm & 0.45 & 2.70 & \pm & 0.15 & 11.2 & \pm & 6.44 \\
\hline Skin & 1.97 & \pm 0.11 & 1.93 & \pm & 0.11 & 2.87 & \pm & 0.13 & 2.85 & \pm & 0.22 & 1.95 & \pm & 0.34 & 6.39 & \pm & 2.36 \\
\hline Brain & 0.70 & \pm 0.12 & 0.49 & \pm & 0.08 & 0.41 & \pm & 0.03 & 0.34 & \pm & 0.06 & 0.32 & \pm & 0.05 & 1.39 & \pm & 0.35 \\
\hline Tumor & 3.56 & $\pm \quad 0.92$ & 4.20 & \pm & 1.02 & 6.52 & \pm & 0.92 & 8.16 & \pm & 0.53 & 6.66 & \pm & 0.91 & 15.6 & \pm & 3.49 \\
\hline \multicolumn{18}{|c|}{ Tumor-to-tissue ratios (rel. u.) } \\
\hline Tumor/Blood & 0.10 & \pm 0.03 & 0.17 & \pm & 0.04 & 0.30 & \pm & 0.05 & 0.51 & \pm & 0.04 & 0.48 & \pm & 0.07 & 1.6 & \pm & 0.5 \\
\hline Tumor/Liver & 0.22 & \pm 0.06 & 0.31 & \pm & 0.08 & 0.42 & \pm & 0.07 & 0.6 & \pm & 0.6 & 0.36 & \pm & 0.06 & 1.1 & \pm & 0.3 \\
\hline Tumor/S. I. & 1.4 & \pm 0.4 & 2.0 & \pm & 0.5 & 2.3 & \pm & 0.4 & 2.9 & \pm & 0.4 & 2.8 & \pm & 0.5 & 4.9 & \pm & 1.4 \\
\hline Tumor/L. I. & 3.8 & \pm 1.3 & 4.6 & \pm & 1.5 & 5.5 & \pm & 0.9 & 5.5 & \pm & 0.6 & 4.1 & \pm & 0.9 & 6.1 & \pm & 1.6 \\
\hline Tumor/Kidney & 0.19 & \pm 0.05 & 0.18 & \pm & 0.05 & 0.18 & \pm & 0.04 & 0.138 & \pm & 0.012 & 0.16 & \pm & 0.05 & 0.24 & \pm & 0.08 \\
\hline Tumor/Muscle & 3.7 & \pm 1.0 & 4.5 & \pm & 1.1 & 5.5 & \pm & 0.8 & 6.6 & \pm & 1.0 & 8 & \pm & 2 & 4.2 & \pm & 3.0 \\
\hline Tumor/Bone & 1.5 & \pm 0.4 & 2.3 & \pm & 0.6 & 3.3 & \pm & 0.7 & 4.2 & \pm & 1.0 & 2.5 & \pm & 0.4 & 1.4 & \pm & 0.9 \\
\hline
\end{tabular}

Table S9-2. Tissue uptake (mean $\% \mathrm{ID} / \mathrm{g} \pm \mathrm{SD}$ ) of ${ }^{18} \mathrm{~F}-\mathrm{AlF}-\mathrm{NO} 2 \mathrm{~A}-\mathrm{cys} \mathrm{WT}$ administered via the lateral tail vein in female, BALB/c mice with orthotopic 4T1 breast cancer allografts implanted into the mammary fat pad. 


\section{F - A IF - N O 2 A - cys W T}

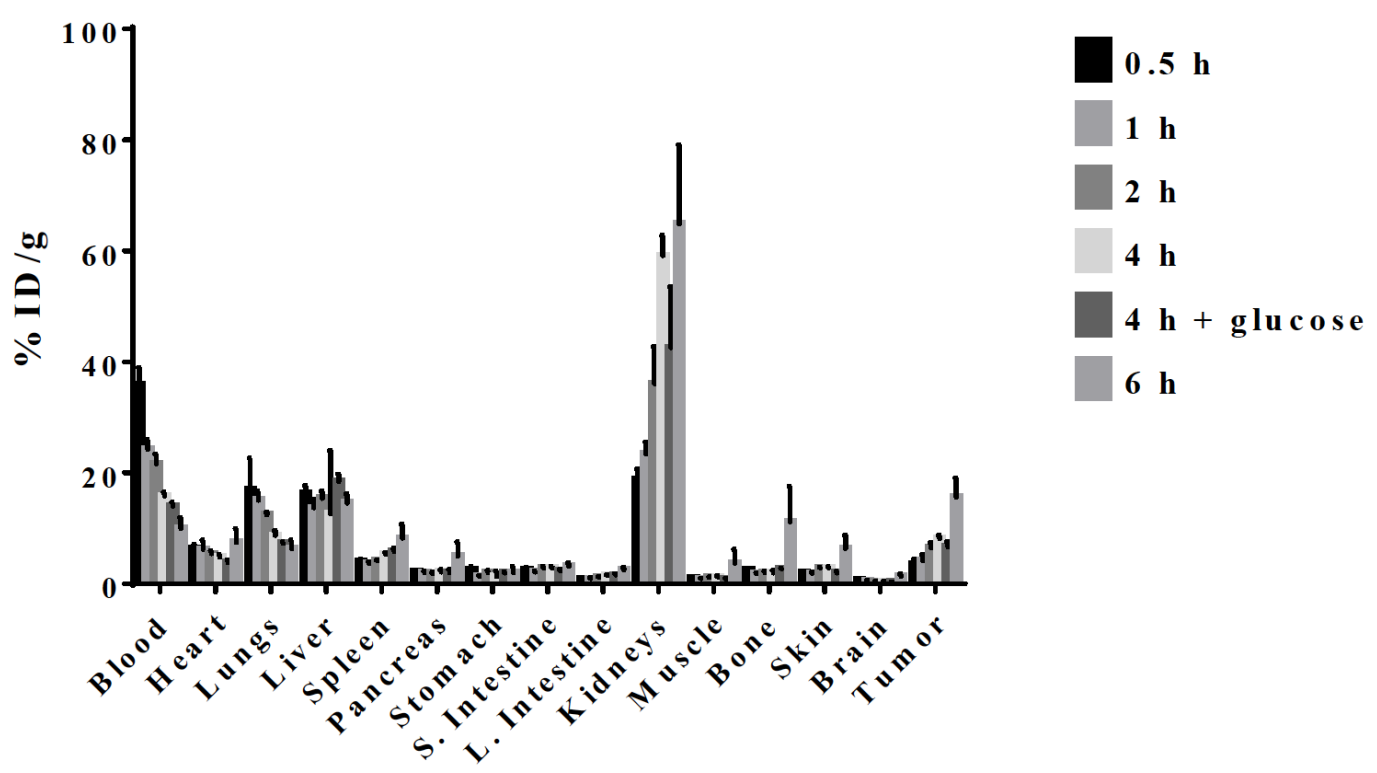

Fig. S9-1. Tissue uptake (mean $\% \mathrm{ID} / \mathrm{g} \pm \mathrm{SD}$ ) of ${ }^{18} \mathrm{~F}-\mathrm{AlF}-\mathrm{NO} 2 \mathrm{~A}-\mathrm{cys} \mathrm{WT}(\mathrm{n}=4)$ administered via the lateral tail vein in female, BALB/c mice with orthotopic 4T1 breast cancer allografts implanted into the mammary fat pad full graph from text. Glucose was administered i.p. 30 min prior to tracer injection. 


\section{BIOSCAN}

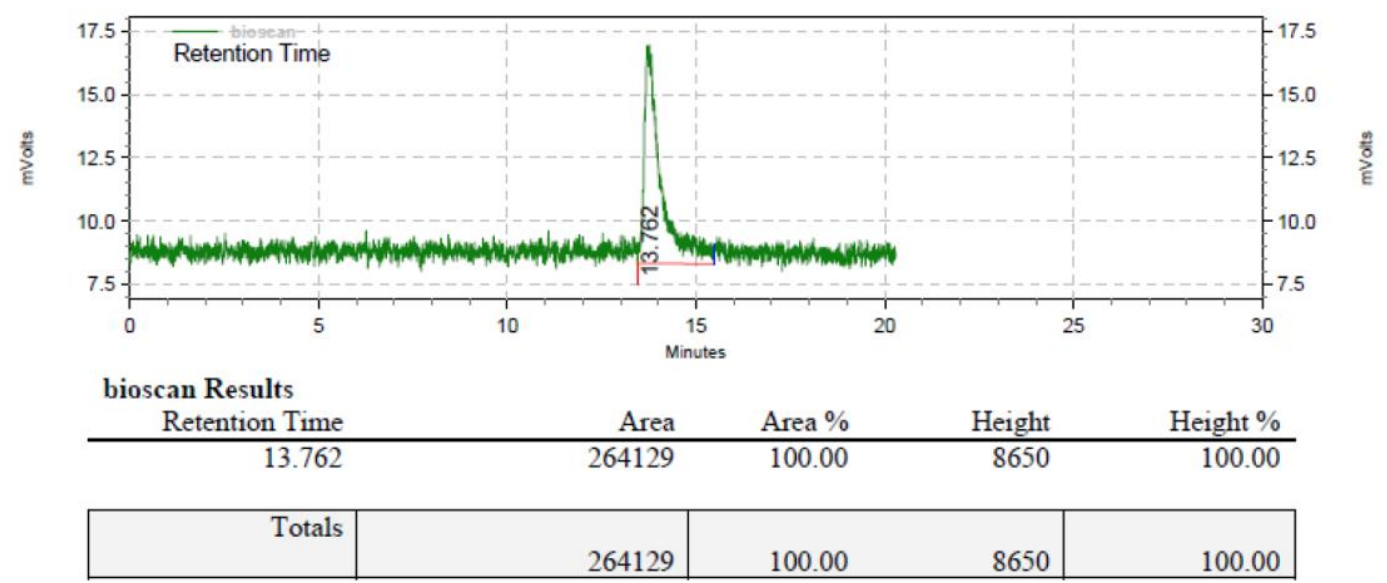

Fig. S9-2. HPLC Chromatogram of the Reconstituted ${ }^{18}$ F-AlF-NO2A-cysWT Injectate 
${ }^{18}$ F-AIF-NO2A-cysVar7

\begin{tabular}{|c|c|c|c|c|c|c|c|c|c|c|c|c|c|c|c|c|c|c|}
\hline \multirow{2}{*}{$\begin{array}{l}\text { Tissue } \\
\text { Blood }\end{array}$} & \multicolumn{3}{|c|}{$0.5 h(n=4)$} & \multicolumn{3}{|c|}{$1 h(n=4)$} & \multicolumn{3}{|c|}{$2 h(n=3)$} & \multicolumn{3}{|c|}{$4 h(n=4)$} & \multicolumn{3}{|c|}{$4 h+$ glucose $(n=3)$} & \multicolumn{3}{|c|}{$6 h(n=5)$} \\
\hline & 3.89 & \pm & 2.41 & 1.92 & \pm & 1.54 & 3.21 & \pm & 2.40 & 0.71 & \pm & 0.37 & 3.12 & \pm & 0.52 & 0.12 & \pm & 0.09 \\
\hline Heart & 0.36 & \pm & 0.05 & 0.24 & \pm & 0.04 & 0.33 & \pm & 0.31 & 0.08 & \pm & 0.02 & 0.13 & \pm & 0.01 & 0.063 & \pm & 0.005 \\
\hline Lungs & 1.83 & \pm & 0.54 & 1.40 & \pm & 0.32 & 1.05 & \pm & 0.44 & 0.40 & \pm & 0.09 & 0.08 & \pm & 0.04 & 0.17 & \pm & 0.03 \\
\hline Liver & 4.97 & \pm & 0.36 & 3.55 & \pm & 0.18 & 4.26 & \pm & 2.00 & 2.15 & \pm & 0.35 & 0.18 & \pm & 0.03 & 2.17 & \pm & 0.22 \\
\hline Spleen & 0.19 & \pm & 0.02 & 0.15 & \pm & 0.01 & 0.18 & \pm & 0.12 & 0.12 & \pm & 0.01 & 1.04 & \pm & 0.12 & 0.11 & \pm & 0.02 \\
\hline Pancreas & 0.17 & \pm & 0.03 & 0.14 & \pm & 0.03 & 0.14 & \pm & 0.08 & 0.07 & \pm & 0.01 & 0.61 & \pm & 0.05 & 0.05 & \pm & 0.01 \\
\hline Stomach & 0.40 & \pm & 0.10 & 0.22 & \pm & 0.01 & 0.25 & \pm & 0.12 & 0.15 & \pm & 0.01 & 52.4 & \pm & 9.57 & 0.17 & \pm & 0.02 \\
\hline S. intestine & 1.63 & \pm & 0.34 & 1.34 & \pm & 0.26 & 1.96 & \pm & 1.54 & 0.96 & \pm & 0.08 & 0.04 & \pm & 0.01 & 0.67 & \pm & 0.08 \\
\hline L. intestine & 0.70 & \pm & 0.05 & 0.51 & \pm & 0.07 & 0.69 & \pm & 0.36 & 0.59 & \pm & 0.06 & 0.02 & \pm & 0.00 & 0.53 & \pm & 0.03 \\
\hline Kidneys & 23.8 & \pm & 4.50 & 40.1 & \pm & 4.20 & 42.2 & \pm & 21.6 & 62.9 & \pm & 3.68 & 0.30 & & 0.04 & 70.5 & \pm & 5.72 \\
\hline Muscle & 0.13 & \pm & 0.03 & 0.06 & \pm & 0.01 & 0.07 & \pm & 0.03 & 0.03 & \pm & 0.01 & 0.03 & \pm & 0.00 & 0.043 & \pm & 0.003 \\
\hline Bone & 0.04 & \pm & 0.02 & 0.02 & \pm & 0.01 & 0.03 & \pm & 0.02 & 0.02 & \pm & 0.01 & 0.40 & \pm & 0.11 & 0.05 & \pm & 0.02 \\
\hline Skin & 0.54 & \pm & 0.06 & 0.35 & \pm & 0.05 & 0.38 & \pm & 0.16 & 0.27 & \pm & 0.03 & 0.81 & \pm & 0.23 & 0.15 & \pm & 0.02 \\
\hline Brain & 0.10 & \pm & 0.03 & 0.04 & \pm & 0.01 & 0.09 & \pm & 0.11 & 0.020 & \pm & 0.003 & 1.04 & \pm & 0.91 & 0.012 & \pm & 0.004 \\
\hline Tumor & 0.21 & \pm & 0.05 & 0.30 & \pm & 0.09 & 0.27 & \pm & 0.09 & 0.53 & \pm & 0.11 & 0.11 & \pm & 0.03 & 0.51 & \pm & 0.10 \\
\hline
\end{tabular}

Table S10-1. Tissue uptake (mean \%ID \pm SD) of ${ }^{18}$ F-AlF-NO2A-cysVar7 administered via the lateral tail vein in female, BALB/c mice with orthotopic 4T1 breast cancer allografts implanted into the mammary fat pad. 


\begin{tabular}{|c|c|c|c|c|c|c|c|c|c|c|c|c|c|c|c|c|c|c|}
\hline \multirow{2}{*}{$\begin{array}{l}\text { Tissue } \\
\text { Blood }\end{array}$} & \multicolumn{3}{|c|}{$0.5 h(n=4)$} & \multicolumn{3}{|c|}{$1 h(n=4)$} & \multicolumn{3}{|c|}{$2 h(n=3)$} & \multicolumn{3}{|c|}{$4 h(n=4)$} & \multicolumn{3}{|c|}{$4 h+$ glucose $(n=3)$} & \multicolumn{3}{|c|}{$6 h(n=5)$} \\
\hline & 14.9 & \pm & 2.24 & 8.51 & \pm & 2.65 & 4.87 & \pm & 0.42 & 1.64 & \pm & 0.18 & 2.37 & \pm & 0.35 & 0.52 & \pm & 0.17 \\
\hline Heart & 3.91 & \pm & 0.56 & 2.34 & \pm & 0.19 & 1.85 & \pm & 0.26 & 0.89 & \pm & 0.12 & 1.21 & \pm & 0.19 & 0.70 & \pm & 0.07 \\
\hline Lungs & 9.45 & \pm & 1.91 & 5.72 & \pm & 0.87 & 3.96 & \pm & 0.21 & 1.66 & \pm & 0.07 & 2.22 & \pm & 0.27 & 0.97 & \pm & 0.20 \\
\hline Liver & 5.89 & \pm & 0.65 & 3.84 & \pm & 0.29 & 3.86 & \pm & 0.33 & 2.90 & \pm & 0.18 & 4.02 & \pm & 0.14 & 2.49 & \pm & 0.13 \\
\hline Spleen & 2.06 & \pm & 0.14 & 1.40 & \pm & 0.17 & 1.38 & \pm & 0.07 & 1.19 & \pm & 0.10 & 1.61 & \pm & 0.21 & 1.05 & \pm & 0.06 \\
\hline Pancreas & 1.29 & \pm & 0.13 & 0.90 & \pm & 0.19 & 0.71 & \pm & 0.03 & 0.49 & \pm & 0.03 & 0.64 & \pm & 0.24 & 0.41 & \pm & 0.05 \\
\hline Stomach & 1.90 & \pm & 0.61 & 0.87 & \pm & 0.09 & 0.94 & \pm & 0.12 & 0.68 & \pm & 0.10 & 0.80 & \pm & 0.09 & 0.39 & \pm & 0.11 \\
\hline S. intestine & 1.73 & \pm & 0.30 & 1.26 & \pm & 0.11 & 1.11 & \pm & 0.09 & 0.91 & \pm & 0.03 & 1.03 & \pm & 0.12 & 0.62 & \pm & 0.08 \\
\hline L. intestine & 1.11 & \pm & 0.06 & 0.77 & \pm & 0.11 & 0.79 & \pm & 0.06 & 0.91 & \pm & 0.12 & 1.05 & \pm & 0.17 & 0.75 & \pm & 0.15 \\
\hline Kidneys & 91.0 & \pm & 20.4 & 139 & \pm & 11.9 & 196 & \pm & 20.3 & 246 & \pm & 25.6 & 198 & \pm & 23.7 & 254 & \pm & 24.4 \\
\hline Muscle & 1.22 & \pm & 0.06 & 0.63 & \pm & 0.06 & 0.59 & \pm & 0.12 & 0.35 & \pm & 0.06 & 0.44 & \pm & 0.06 & 0.32 & \pm & 0.05 \\
\hline Bone & 1.03 & \pm & 0.13 & 0.79 & \pm & 0.17 & 0.78 & \pm & 0.37 & 0.60 & \pm & 0.12 & 0.86 & \pm & 0.14 & 0.79 & \pm & 0.08 \\
\hline Skin & 3.23 & \pm & 0.40 & 2.19 & \pm & 0.23 & 1.93 & \pm & 0.13 & 1.59 & \pm & 0.10 & 1.82 & \pm & 0.12 & 1.45 & \pm & 0.16 \\
\hline Brain & 0.29 & \pm & 0.04 & 0.14 & \pm & 0.03 & 0.10 & \pm & 0.01 & 0.05 & \pm & 0.01 & 0.08 & \pm & 0.00 & 0.03 & \pm & 0.01 \\
\hline Tumor & 4.52 & \pm & 0.54 & 5.04 & \pm & 0.94 & 6.60 & \pm & 1.22 & 8.61 & \pm & 1.21 & 6.20 & \pm & 1.55 & 7.86 & \pm & 1.40 \\
\hline \multicolumn{19}{|c|}{ Tumor-to-tissue ratios (rel. u.) } \\
\hline Tumor/Blood & 0.30 & \pm & 0.06 & 0.6 & \pm & 0.2 & 1.4 & \pm & 0.3 & 5.3 & \pm & 0.9 & 2.6 & \pm & 0.8 & 15 & \pm & 6 \\
\hline Tumor/Liver & 0.77 & \pm & 0.12 & 1.3 & \pm & 0.3 & 1.7 & \pm & 0.3 & 3.0 & \pm & 0.5 & 1.5 & \pm & 0.4 & 3.2 & \pm & 0.6 \\
\hline Tumor/S. I. & 2.6 & \pm & 0.6 & 4.0 & \pm & 0.8 & 6.0 & \pm & 1.2 & 9.5 & \pm & 1.4 & 6.0 & \pm & 1.7 & 13 & \pm & 3 \\
\hline Tumor/L. I. & 4.1 & \pm & 0.5 & 6.5 & \pm & 1.5 & 8.3 & \pm & 1.6 & 9.5 & \pm & 1.8 & 5.9 & \pm & 1.8 & 10 & \pm & 3 \\
\hline Tumor/Kidney & 0.050 & \pm & 0.013 & 0.036 & \pm & 0.007 & 0.034 & \pm & 0.007 & 0.035 & \pm & 0.006 & 0.031 & \pm & 0.009 & 0.031 & \pm & 0.006 \\
\hline Tumor/Muscle & 3.7 & \pm & 0.5 & 8.0 & \pm & 1.7 & 11 & \pm & 3 & 24 & \pm & 5 & 14 & \pm & 4 & 24 & \pm & 6 \\
\hline Tumor/Bone & 4.4 & \pm & 0.8 & 6.4 & \pm & 1.8 & 8 & \pm & 4 & 14 & \pm & 4 & 7 & \pm & 2 & 10 & \pm & 2 \\
\hline
\end{tabular}

Table S10-2. Tissue uptake (mean \% ID/g \pm SD) of ${ }^{18} \mathrm{~F}-\mathrm{AlF}-\mathrm{NO} 2 \mathrm{~A}-\mathrm{cys}$ ar7 administered via the lateral tail vein in female, BALB/c mice with orthotopic 4T1 breast cancer allografts implanted into the mammary fat pad. 


\section{${ }^{18}$ F - A IF - N O 2 A - cy s V a r 7}

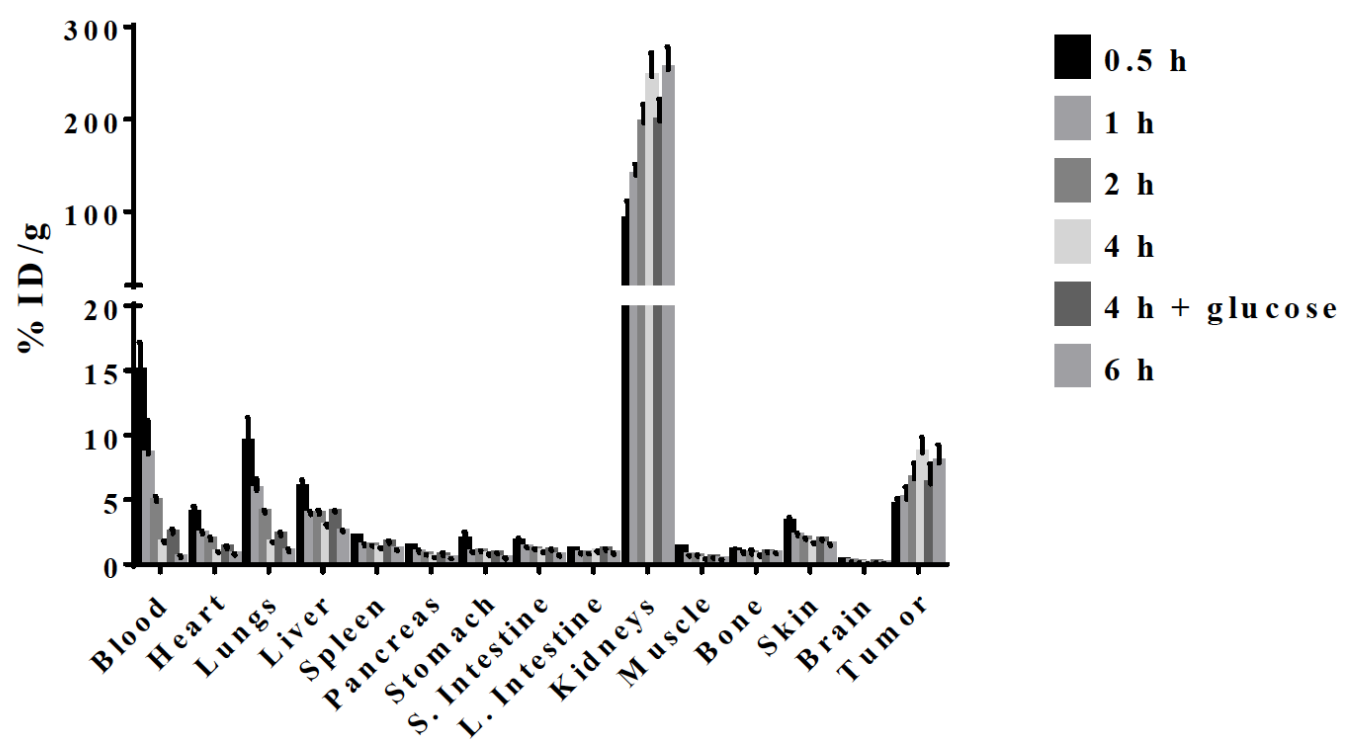

Fig. S10-1. Tissue uptake (mean $\% \mathrm{ID} / \mathrm{g} \pm \mathrm{SD}$ ) of ${ }^{18} \mathrm{~F}-\mathrm{AlF}-\mathrm{NO} 2 \mathrm{~A}-\mathrm{cys} \operatorname{Var} 7(\mathrm{n}=3-5)$ administered via the lateral tail vein in female, BALB/c mice with orthotopic 4T1 breast cancer allografts implanted into the mammary fat pad full graph from text. Glucose was administered i.p. 30 min prior to tracer injection. 


\section{BIOSCAN}

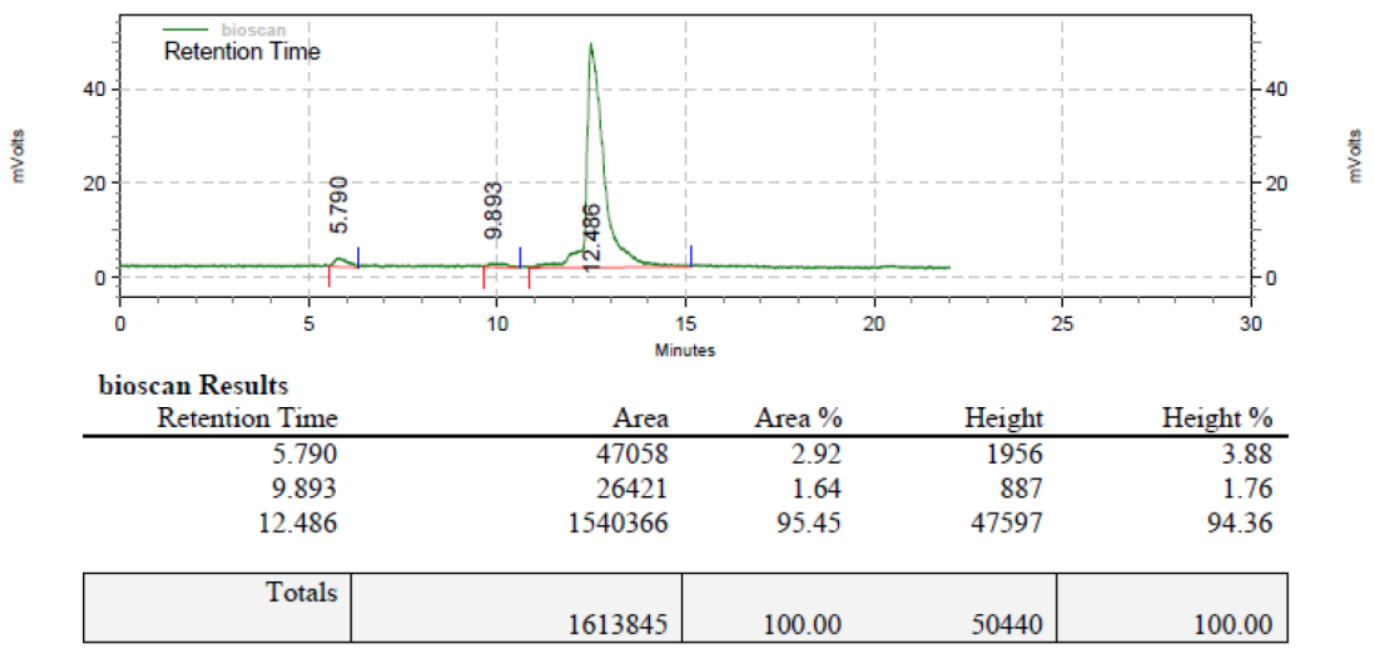

Fig. S10-2. HPLC Chromatogram of the Reconstituted ${ }^{18}$ F-AlF-NO2A-cysVar7 Injectate 


\section{${ }^{64} \mathrm{Cu}-\mathrm{NO2A}-\mathrm{cys} \operatorname{Var} 3$}

\begin{tabular}{|c|c|c|c|c|c|c|c|c|c|c|c|c|c|c|c|c|c|c|c|c|c|c|c|c|}
\hline \multirow{2}{*}{$\begin{array}{l}\text { Tissue } \\
\text { Blood }\end{array}$} & \multicolumn{3}{|c|}{$1 \mathrm{~h}(\mathrm{n}=5)$} & \multicolumn{3}{|c|}{$2 \mathrm{~h}(\mathrm{n}=5)$} & \multicolumn{3}{|c|}{$4 \mathrm{~h}(\mathrm{n}=5)$} & \multicolumn{3}{|c|}{$\begin{array}{c}4 \mathrm{~h}+\text { glucose } \\
(\mathrm{n}=5)\end{array}$} & \multicolumn{3}{|c|}{12 h $(n=5)$} & \multicolumn{3}{|c|}{$24 \mathrm{~h}(\mathrm{n}=8)$} & \multicolumn{3}{|c|}{$36 \mathrm{~h}(\mathrm{n}=3)$} & \multicolumn{3}{|c|}{$48 h(n=4)$} \\
\hline & 9.25 & \pm & 4.66 & 4.14 & \pm & 3.47 & 3.61 & $\overline{ \pm 3}$ & 3.04 & 2.67 & \pm & 1.31 & 1.56 & \pm & 0.41 & 82 & \pm & 0.85 & 1.10 & \pm & 0.24 & 0.64 & \pm 0 & 0.07 \\
\hline Heart & 0.76 & \pm & 0.11 & 0.70 & \pm & 0.13 & 0.59 & \pm 0 & 0.07 & 0.70 & \pm & 0.09 & 0.48 & \pm & 0.06 & 0.49 & \pm & 0.06 & 0.45 & \pm & 0.02 & 0.48 & \pm 0 & 0.04 \\
\hline Lungs & 6.41 & \pm & 1.03 & 4.28 & \pm & 1.18 & 4.44 & \pm 0 & 0.18 & 3.74 & \pm & 1.28 & 1.90 & \pm( & 0.50 & 1.39 & \pm( & 0.17 & 1.51 & \pm & 0.11 & 1.12 & \pm 0 & 0.10 \\
\hline Liver & 11.9 & \pm & 0.47 & 10.3 & \pm & 1.55 & 10.5 & \pm 1 & 1.03 & 12.5 & \pm & 2.43 & 10.6 & \pm & 0.66 & 12.3 & \pm & 1.75 & 14.5 & \pm & 0.14 & 13.5 & \pm 0 & 0.35 \\
\hline Spleen & 0.37 & \pm & 0.02 & 0.40 & \pm & 0.03 & 0.41 & \pm 0 & 0.05 & 0.45 & \pm & 0.08 & 0.42 & \pm( & 0.05 & 0.55 & \pm( & 0.08 & 0.73 & \pm & 0.17 & 0.68 & \pm 0 & 0.13 \\
\hline Pancreas & 0.46 & \pm & 0.04 & 0.38 & \pm & 0.05 & 0.37 & \pm 0 & 0.07 & 0.36 & \pm & 0.03 & 0.29 & \pm & 0.01 & 0.40 & \pm & 0.07 & 0.42 & \pm & 0.03 & 0.43 & \pm 0 & 0.06 \\
\hline Stomach & 0.56 & \pm & 0.07 & 0.55 & \pm & 0.07 & 0.61 & \pm 0 & 0.08 & 0.54 & \pm & 0.11 & 0.86 & \pm( & 0.15 & 0.97 & \pm( & 0.20 & 1.01 & \pm & 0.02 & 1.17 & \pm 0 & 0.13 \\
\hline S. intestine & 4.32 & \pm & 0.35 & 3.65 & \pm & 0.52 & 4.31 & \pm 0 & 0.40 & 3.78 & \pm & 0.79 & 5.44 & \pm & 0.38 & 5.47 & \pm & 0.75 & 5.26 & \pm & 0.21 & 4.90 & \pm 0 & 0.31 \\
\hline L. intestine & 1.32 & \pm & 0.19 & 1.77 & \pm & 0.16 & 2.45 & \pm 0 & 0.16 & 2.29 & \pm & 0.36 & 3.66 & \pm & 0.20 & 5.44 & \pm & 0.87 & 4.11 & \pm & 0.04 & 5.50 & \pm 0 & 0.43 \\
\hline Kidneys & 6.92 & \pm & 0.17 & 7.45 & \pm & 0.44 & 8.52 & \pm 0 & 0.69 & 8.13 & \pm & 0.80 & 6.05 & \pm & 0.60 & 3.98 & \pm & 0.52 & 3.94 & \pm & 0.20 & 3.15 & \pm 0 & 0.21 \\
\hline Muscle & 0.11 & \pm & 0.04 & 0.13 & \pm & 0.02 & 0.15 & \pm 0 & 0.01 & 0.14 & \pm & 0.0 & 0.12 & \pm( & 0.04 & 0.11 & \pm( & 0.04 & 0.12 & \pm & 0.03 & 0.09 & \pm 0 & 0.03 \\
\hline Bone & 0.07 & \pm & 0.03 & 0.05 & \pm & 0.02 & 0.04 & \pm 0 & 0.01 & 0.05 & \pm & 0.03 & 0.13 & \pm & 0.06 & 0.08 & \pm & 0.05 & 0.09 & \pm & 0.00 & 0.09 & \pm 0 & 0.04 \\
\hline Skin & 0.37 & \pm & 0.04 & 0.46 & \pm & 0.11 & 0.64 & \pm 0 & 0.21 & 0.44 & \pm & 0.13 & 0.43 & \pm & 0.08 & 0.33 & \pm & 0.12 & 0.28 & \pm & 0.05 & 0.30 & \pm 0 & 0.06 \\
\hline Brain & 0.20 & \pm & 0.04 & 0.18 & \pm & 0.04 & 0.13 & \pm 0 & 0.03 & 0.17 & \pm & 0.02 & 0.17 & \pm & 0.03 & 0.16 & \pm & 0.02 & 0.20 & \pm & 0.01 & 0.22 & \pm 0 & 0.02 \\
\hline Tumor & 0.19 & \pm & 0.05 & 0.32 & \pm & 0.09 & 0.53 & \pm 0 & 0.15 & 0.56 & \pm & 0.12 & 1.12 & \pm & 0.41 & 1.67 & \pm & 0.87 & 2.37 & \pm & 0.49 & 2.18 & & 0.33 \\
\hline
\end{tabular}

Table S11-1. Tissue uptake (mean $\%$ ID $\pm \mathrm{SD}$ ) of ${ }^{64} \mathrm{Cu}-\mathrm{NO} 2 \mathrm{~A}-\mathrm{cys}$ Var3 administered via the lateral tail vein in female, BALB/c mice with orthotopic 4T1 breast cancer allografts implanted into the mammary fat pad. 


\begin{tabular}{|c|c|c|c|c|c|c|c|c|c|c|c|c|c|c|c|c|c|c|c|c|c|c|c|c|}
\hline \multirow{2}{*}{$\begin{array}{l}\text { Tissue } \\
\text { Blood }\end{array}$} & \multicolumn{3}{|c|}{$1 \mathrm{~h}(\mathrm{n}=5)$} & \multicolumn{3}{|c|}{$2 \mathrm{~h}(\mathrm{n}=5)$} & \multicolumn{3}{|c|}{$4 \mathrm{~h}(\mathrm{n}=5)$} & \multicolumn{3}{|c|}{$\begin{array}{c}4 \mathrm{~h}+\text { glucose } \\
(\mathrm{n}=5)\end{array}$} & \multicolumn{3}{|c|}{$12 \mathrm{~h}(\mathrm{n}=5)$} & \multicolumn{3}{|c|}{$24 \mathrm{~h}(\mathrm{n}=8)$} & \multicolumn{3}{|c|}{$36 \mathrm{~h}(\mathrm{n}=3)$} & \multicolumn{3}{|c|}{$48 \mathrm{~h}(\mathrm{n}=4)$} \\
\hline & 27.8 & \pm & 2.54 & 20.0 & \pm & 2.25 & 15.4 & \pm & 1.76 & 23.2 & \pm & 4.10 & 9.88 & \pm & 1.30 & 6.77 & \pm & 0.41 & 4.13 & & 0.51 & 3.34 & \pm & 0.80 \\
\hline Heart & 8.24 & \pm & 1.29 & 6.48 & \pm & 1.03 & 5.12 & \pm & 0.63 & 7.26 & \pm & 1.26 & 4.42 & \pm & 0.54 & 4.80 & \pm & 0.50 & 4.37 & \pm & 0.29 & 5.10 & \pm & 0.72 \\
\hline Lungs & 24.6 & \pm & 3.62 & 16.0 & \pm & 2.56 & 14.7 & \pm & 0.93 & 17.7 & \pm & 4.09 & 8.17 & \pm & 1.08 & 7.74 & \pm & 0.95 & 6.19 & \pm & 0.41 & 7.51 & \pm & 1.45 \\
\hline Liver & 14.1 & \pm & 0.86 & 11.4 & \pm & 1.57 & 10.4 & \pm & 0.63 & 14.3 & \pm & 2.63 & 10.3 & \pm & 1.66 & 13.6 & \pm & 1.58 & 13.0 & \pm & 0.40 & 16.2 & \pm & 2.35 \\
\hline Spleen & 4.09 & \pm & 0.45 & 3.39 & \pm & 0.22 & 3.45 & \pm & 0.35 & 5.19 & \pm & 0.86 & 4.32 & \pm & 0.62 & 5.07 & \pm & 0.40 & 5.85 & \pm & 0.37 & 4.90 & \pm & 0.29 \\
\hline Pancreas & 3.27 & \pm & 0.39 & 2.36 & \pm & 0.15 & 2.31 & \pm & 0.25 & 3.04 & \pm & 0.28 & 2.46 & \pm & 0.34 & 2.68 & \pm & 0.25 & 2.92 & \pm & 0.11 & 3.24 & \pm & 0.26 \\
\hline Stomach & 2.24 & \pm & 0.41 & 2.04 & \pm & 0.35 & 1.27 & \pm & 0.35 & 1.84 & \pm & 0.35 & 1.09 & \pm & 0.25 & 3.09 & \pm & 1.19 & 1.56 & \pm & 0.06 & 3.39 & \pm & 1.03 \\
\hline S. intestine & 3.48 & \pm & 0.29 & 2.98 & \pm & 0.38 & 2.68 & \pm & 0.24 & 3.49 & \pm & 0.61 & 3.92 & \pm & 0.36 & 4.91 & \pm & 0.64 & 4.14 & \pm & 0.17 & 5.37 & \pm & 0.15 \\
\hline L. intestine & 2.02 & \pm & 0.29 & 2.08 & \pm & 0.12 & 2.99 & \pm & 0.20 & 3.68 & \pm & 0.62 & 4.31 & \pm & 0.62 & 6.69 & \pm & 1.33 & 4.77 & \pm & 0.07 & 7.52 & \pm & 0.67 \\
\hline Kidneys & 25.3 & \pm & 1.99 & 23.1 & \pm & 1.42 & 24.0 & \pm & 2.78 & 33.1 & \pm & 6.29 & 20.5 & \pm & 2.44 & 13.3 & \pm & 1.14 & 12.4 & \pm & 0.63 & 10.6 & \pm & 1.84 \\
\hline Muscle & 1.19 & \pm & 0.10 & 1.03 & \pm & 0.10 & 1.10 & \pm & 0.15 & 1.39 & \pm & 0.28 & 1.30 & \pm & 0.18 & 1.20 & \pm & 0.13 & 1.06 & \pm & 0.18 & 1.01 & \pm & 0.23 \\
\hline Bone & 2.26 & \pm & 0.29 & 1.18 & \pm & 0.22 & 1.10 & \pm & 0.14 & 1.88 & \pm & 0.42 & 2.36 & \pm & 0.31 & 1.78 & \pm & 0.23 & 2.10 & \pm & 0.10 & 1.88 & \pm & 0.30 \\
\hline Skin & 2.62 & \pm & 0.14 & 2.63 & \pm & 0.49 & 3.43 & \pm & 0.34 & 3.27 & \pm & 0.62 & 3.45 & \pm & 0.54 & 3.17 & \pm & 0.25 & 2.83 & \pm & 0.11 & 2.93 & \pm & 0.58 \\
\hline Brain & 0.63 & \pm & 0.09 & 0.48 & \pm & 0.09 & 0.35 & \pm & 0.07 & 0.54 & \pm & 0.07 & 0.42 & \pm & 0.08 & 0.42 & \pm & 0.0 & 0.49 & \pm & 0.03 & 0.57 & \pm & 0.08 \\
\hline Tumor & 4.19 & \pm & 0.65 & 5.43 & \pm & 0.93 & 8.21 & \pm & 0.86 & 8.81 & \pm & 1.78 & 14.5 & \pm & 2.45 & 19.6 & \pm & 2.34 & 19.2 & \pm & 1.88 & 16.2 & \pm & 1.90 \\
\hline \multicolumn{25}{|c|}{ Tumor-to-tissue ratios (rel. u.) } \\
\hline Tumor/Blood & 0.15 & \pm & 0.03 & 0.27 & \pm & 0.06 & 0.53 & \pm & 0.08 & 0.38 & \pm & 0.10 & 1.5 & \pm & 0.3 & 2.9 & \pm & 0.4 & 4.7 & \pm & 0.7 & 4.8 & \pm & 1.3 \\
\hline Tumor/Liver & 0.30 & \pm & 0.05 & 0.47 & \pm & 0.10 & 0.79 & \pm & 0.10 & 0.61 & \pm & 0.17 & 1.4 & \pm & 0.3 & 1.4 & \pm & 0.2 & 1.47 & \pm & 0.15 & 1.00 & \pm & 0.19 \\
\hline Tumor/S. I. & 1.2 & \pm & 0.2 & 1.8 & \pm & 0.4 & 3.1 & \pm & 0.4 & 2.5 & \pm & 0.7 & 3.7 & \pm & 0.7 & 4.0 & \pm & 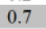 & 4.6 & \pm & 0.5 & 3.0 & $I$ & 0.4 \\
\hline Tumor/L. I. & 2.1 & \pm & 0.4 & 2.6 & \pm & 0.5 & 2.7 & \pm & 0.3 & 2.4 & \pm & 0.6 & 3.4 & \pm & 0.7 & 2.9 & \pm & 0.7 & 4.0 & \pm & 0.4 & 2.2 & \pm & 0.3 \\
\hline Tumor/Kidn & 0.17 & \pm & 0.03 & 0.23 & \pm & 0.04 & 0.34 & \pm & 0.05 & 0.27 & \pm & 0.07 & 0.71 & \pm & 0.15 & 1.5 & \pm & 0.2 & 1.55 & \pm & 0.17 & 1.5 & \pm & 0.3 \\
\hline Tumor/Muscle & 3.5 & \pm & 0.6 & 5.3 & \pm & 1.0 & 7.4 & \pm & 1.3 & 6.3 & \pm & 1.8 & 11 & \pm & 2 & 16 & \pm & 3 & 18 & \pm & 3 & 16 & \pm & 4 \\
\hline Tumor/Bone & 1.9 & \pm & 0.4 & 4.6 & \pm & 1.2 & 7.4 & \pm & 1.2 & 4.7 & \pm & 1.4 & 6.2 & \pm & 1.3 & 11.0 & \pm & 1.9 & 9.1 & \pm & 1.0 & 8.6 & \pm & 1.7 \\
\hline
\end{tabular}

Table S11-2. Tissue uptake (mean $\% \mathrm{ID} / \mathrm{g} \pm \mathrm{SD}$ ) of ${ }^{64} \mathrm{Cu}-\mathrm{NO} 2 \mathrm{~A}-\mathrm{cys}$ Var3 administered via the lateral tail vein in female, $\mathrm{BALB} / \mathrm{c}$ mice with orthotopic $4 \mathrm{~T} 1$ breast cancer allografts implanted into the mammary fat pad. 


\section{${ }^{64} \mathrm{Cu}-\mathrm{N} O 2 \mathrm{~A}-\mathrm{cys} \mathrm{V}$ ar 3}

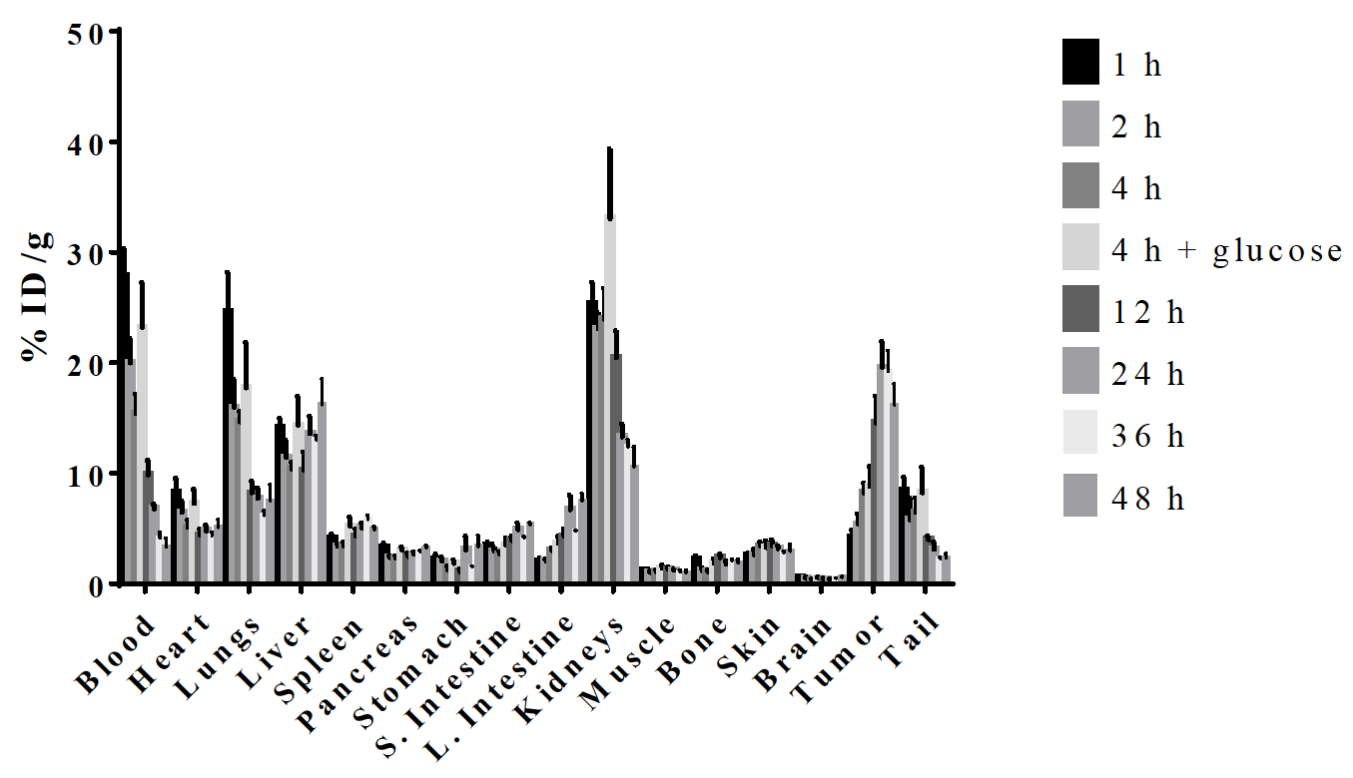

Fig. S11-1. Tissue uptake (mean \%ID/g $\pm \mathrm{SD})$ of ${ }^{64} \mathrm{Cu}-\mathrm{NO} 2 \mathrm{~A}-\mathrm{cys} \operatorname{Var} 3(\mathrm{n}=3-8)$ administered via the lateral tail vein in female, BALB/c mice with orthotopic $4 \mathrm{~T} 1$ breast cancer allografts implanted into the mammary fat pad full graph from text. Glucose was administered i.p. 30 min prior to tracer injection. 


\section{BIOSCAN}

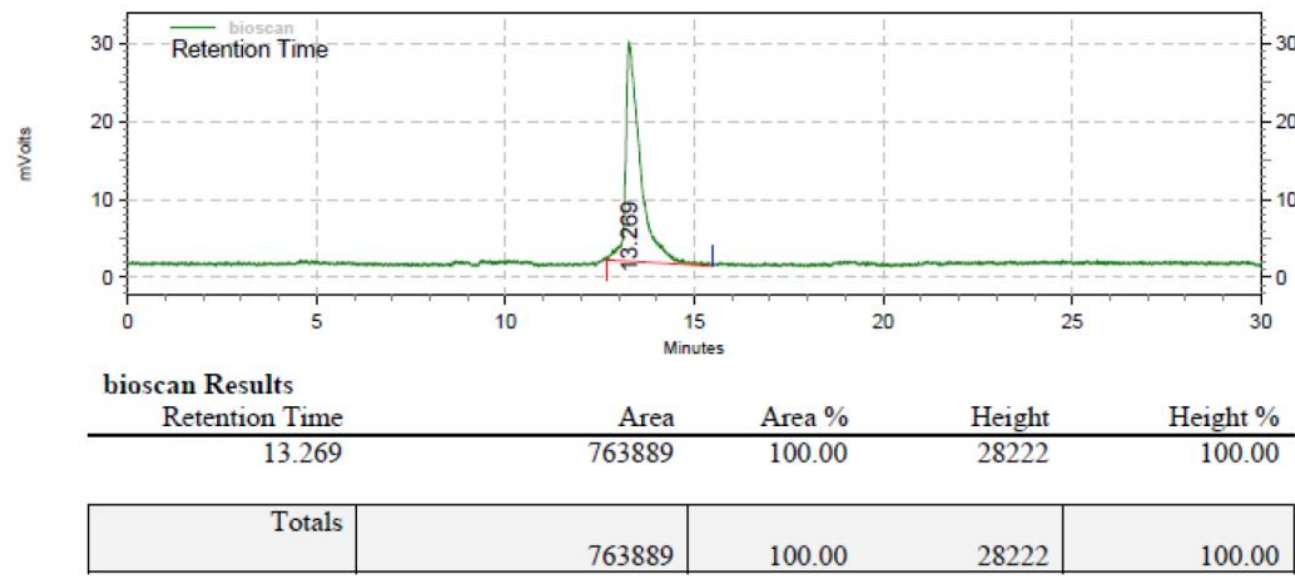

Fig. S11-2. HPLC Chromatogram of the Reconstituted ${ }^{64} \mathrm{Cu}-\mathrm{NO} 2 \mathrm{~A}-\mathrm{cys} V$ ar3 Injectate 
${ }^{18}$ F-AIF-NO2A-cysVar3

\begin{tabular}{|c|c|c|c|c|c|c|c|c|c|c|c|c|c|c|c|c|c|c|c|c|c|}
\hline \multirow{2}{*}{$\begin{array}{l}\text { Tissue } \\
\text { Blood }\end{array}$} & \multicolumn{3}{|c|}{$0.5 \mathrm{~h}(\mathrm{n}=5)$} & \multicolumn{3}{|c|}{$1 \mathrm{~h}(\mathrm{n}=5)$} & \multicolumn{3}{|c|}{$2 h(n=5)$} & \multicolumn{3}{|c|}{$4 h(n=10)$} & \multicolumn{3}{|c|}{$4 h+$ glucose $(n=4)$} & \multicolumn{3}{|c|}{$6 h(n=5)$} & \multicolumn{3}{|c|}{$8 h(n=5)$} \\
\hline & 5.50 & \pm & 3.19 & 9.36 & \pm & 8.75 & 2.96 & \pm & 1.05 & 5.81 & \pm & 2.46 & 3.71 & \pm & 1.51 & 2.46 & \pm & 0.99 & 4.71 & \pm & 2.12 \\
\hline Heart & 1.26 & \pm & 0.16 & 1.08 & \pm & 0.23 & 0.82 & \pm & 0.16 & 0.63 & \pm & 0.12 & 0.66 & \pm & 0.26 & 0.54 & \pm & 0.08 & 0.55 & \pm & 0.06 \\
\hline Lungs & 11.2 & \pm & 0.83 & 8.53 & \pm & 2.35 & 7.02 & \pm & 0.94 & 2.27 & \pm & 1.04 & 3.98 & \pm & 1.98 & 3.55 & \pm & 0.64 & 1.06 & \pm & 0.18 \\
\hline Liver & 11.7 & \pm & 1.93 & 12.1 & \pm & 0.61 & 9.57 & \pm & 1.32 & 8.00 & \pm & 0.96 & 6.79 & \pm & 2.07 & 7.91 & \pm & 0.46 & 7.14 & \pm & 0.67 \\
\hline Spleen & 0.58 & \pm & 0.03 & 0.49 & \pm & 0.03 & 0.41 & \pm & 0.02 & 0.45 & \pm & 0.12 & 0.31 & \pm & 0.09 & 0.36 & \pm & 0.03 & 0.51 & \pm & 0.05 \\
\hline Pancreas & 0.70 & \pm & 0.11 & 0.49 & \pm & 0.18 & 0.41 & \pm & 0.05 & 0.38 & \pm & 0.08 & 0.26 & \pm & 0.05 & 0.32 & \pm & 0.02 & 0.26 & \pm & 0.03 \\
\hline Stomach & 0.91 & \pm & 0.25 & 0.55 & \pm & 0.08 & 0.53 & \pm & 0.08 & 0.52 & \pm & 0.06 & 0.38 & \pm & 0.13 & 0.49 & \pm & 0.09 & 0.44 & \pm & 0.06 \\
\hline S. intestine & 3.33 & \pm & 0.44 & 3.91 & \pm & 0.71 & 3.47 & \pm & 0.69 & 2.09 & \pm & 0.30 & 2.29 & \pm & 0.66 & 1.90 & \pm & 0.12 & 2.28 & \pm & 0.12 \\
\hline L. intestine & 1.57 & \pm & 0.17 & 1.48 & \pm & 0.08 & 1.50 & \pm & 0.28 & 1.67 & \pm & 0.61 & 1.54 & \pm & 0.58 & 1.48 & \pm & 0.31 & 1.10 & \pm & 0.11 \\
\hline Kidneys & 6.69 & \pm & 0.67 & 8.56 & \pm & 0.50 & 11.2 & \pm & 0.61 & 10.1 & \pm & 1.35 & 12.2 & \pm & 2.88 & 10.6 & \pm & 0.51 & 15.3 & \pm & 1.74 \\
\hline Muscle & 0.13 & \pm & 0.05 & 0.16 & \pm & 0.03 & 0.11 & \pm & 0.03 & 0.16 & \pm & 0.04 & 0.09 & \pm & 0.05 & 0.15 & \pm & 0.02 & 0.19 & \pm & 0.07 \\
\hline Bone & 0.14 & \pm & 0.04 & 0.06 & \pm & 0.03 & 0.12 & \pm & 0.08 & 0.08 & \pm & 0.06 & 0.07 & \pm & 0.01 & 0.11 & \pm & 0.01 & 0.11 & \pm & 0.00 \\
\hline Skin & 0.28 & \pm & 0.09 & 0.42 & \pm & 0.08 & 0.61 & \pm & 0.16 & 0.29 & \pm & 0.16 & 0.37 & \pm & 0.12 & 0.36 & \pm & 0.11 & 0.31 & \pm & 0.07 \\
\hline Brain & 0.34 & \pm & 0.05 & 0.22 & \pm & 0.02 & 0.21 & \pm & 0.04 & 0.17 & \pm & 0.05 & 0.12 & \pm & 0.03 & 0.17 & \pm & 0.03 & 0.19 & \pm & 0.05 \\
\hline Tumor & 0.29 & \pm & 0.09 & 0.33 & \pm & 0.06 & 0.47 & \pm & 0.11 & 0.79 & \pm & 0.22 & 0.56 & \pm & 0.20 & 1.13 & \pm & 0.21 & 1.97 & \pm & 0.99 \\
\hline
\end{tabular}

Table S12-1. Tissue uptake (mean \%ID \pm SD) of ${ }^{18}$ F-AlF-NO2A-cysVar3

administered via the lateral tail vein in female, BALB/c mice with orthotopic 4T1 breast cancer allografts implanted into the mammary fat pad. 


\begin{tabular}{|c|c|c|c|c|c|c|c|c|c|c|c|c|c|c|c|c|c|c|c|c|c|}
\hline \multirow{2}{*}{$\begin{array}{l}\text { Tissue } \\
\text { Blood }\end{array}$} & \multicolumn{3}{|c|}{$0.5 \mathrm{~h}(\mathrm{n}=5)$} & \multicolumn{3}{|c|}{$1 \mathrm{~h}(\mathrm{n}=5)$} & \multicolumn{3}{|c|}{$2 h(n=5)$} & \multicolumn{3}{|c|}{$4 h(n=10)$} & \multicolumn{3}{|c|}{$4 h+$ glucose $(n=4)$} & \multicolumn{3}{|c|}{$6 \mathrm{~h}(\mathrm{n}=5)$} & \multicolumn{3}{|c|}{$8 \mathrm{~h}(\mathrm{n}=5)$} \\
\hline & 35.0 & \pm 2 & 2.37 & 32.3 & \pm & 2.37 & 22.3 & \pm & 2.67 & 20.8 & \pm & 1.88 & 15.5 & \pm & 2.06 & 16.5 & \pm & 1.68 & 16.1 & \pm & 1.87 \\
\hline Heart & 10.7 & \pm( & 0.82 & 9.41 & \pm & 1.13 & 7.02 & \pm & 0.87 & 6.29 & \pm & 1.03 & 5.60 & \pm & 0.89 & 5.37 & \pm & 0.62 & 5.91 & \pm & 0.44 \\
\hline Lungs & 36.3 & \pm & 4.37 & 28.3 & \pm & 3.61 & 22.2 & \pm & 2.94 & 11.8 & \pm & 3.79 & 16.6 & \pm & 1.35 & 13.7 & \pm & 1.97 & 7.03 & \pm & 0.53 \\
\hline Liver & 12.6 & \pm( & 0.55 & 12.9 & \pm & 0.55 & 9.58 & \pm & 1.52 & 9.06 & \pm & 1.24 & 8.01 & \pm & 1.18 & 8.85 & \pm & 0.69 & 8.80 & \pm & 0.25 \\
\hline Spleen & 4.99 & \pm( & 0.30 & 4.00 & \pm & 0.12 & 3.56 & \pm & 0.35 & 3.73 & \pm & 0.66 & 2.91 & \pm & 0.35 & 3.36 & \pm & 0.23 & 4.47 & \pm & 0.60 \\
\hline Pancreas & 3.90 & \pm( & 0.35 & 3.35 & \pm & 0.30 & 2.49 & \pm & 0.25 & 2.44 & \pm & 0.20 & 1.87 & \pm & 0.15 & 2.17 & \pm & 0.20 & 2.09 & \pm & 0.27 \\
\hline Stomach & 1.92 & \pm( & 0.44 & 1.68 & \pm & 0.23 & 1.31 & \pm & 0.16 & 1.29 & \pm & 0.62 & 1.11 & \pm & 0.37 & 1.36 & \pm & 0.43 & 0.86 & \pm & 0.23 \\
\hline S. intestine & 2.89 & \pm( & 0.22 & 3.24 & \pm & 0.23 & 2.39 & \pm & 0.25 & 2.06 & \pm & 0.28 & 1.78 & \pm & 0.23 & 1.79 & \pm & 0.12 & 2.19 & \pm & 0.29 \\
\hline L. intestine & 1.76 & \pm( & 0.17 & 2.14 & \pm & 0.10 & 2.05 & \pm & 0.21 & 2.07 & \pm & 0.63 & 2.05 & \pm & 0.40 & 2.04 & \pm & 0.41 & 1.57 & \pm & 0.26 \\
\hline Kidneys & 18.7 & \pm & 1.45 & 26.2 & \pm & 2.11 & 33.7 & \pm & 3.00 & 34.3 & \pm & 6.91 & 41.0 & \pm & 1.54 & 35.2 & \pm & 1.25 & 53.0 & \pm & 6.26 \\
\hline Muscle & 1.01 & \pm( & 0.15 & 1.23 & \pm & 0.09 & 1.07 & \pm & 0.14 & 1.55 & \pm & 0.27 & 0.99 & \pm & 0.39 & 1.47 & \pm & 0.17 & 1.61 & \pm & 0.39 \\
\hline Bone & 2.14 & \pm( & 0.21 & 1.65 & \pm & 0.31 & 1.81 & \pm & 0.54 & 1.66 & \pm & 0.61 & 1.44 & \pm & 0.15 & 2.32 & \pm & 0.12 & 2.37 & \pm & 0.17 \\
\hline Skin & 2.17 & \pm( & 0.21 & 2.63 & \pm & 0.23 & 3.06 & \pm & 0.39 & 2.92 & \pm & 0.71 & 2.70 & \pm & 0.45 & 3.73 & \pm & 0.34 & 3.37 & \pm & 0.20 \\
\hline Brain & 0.85 & \pm( & 0.05 & 0.60 & \pm & 0.05 & 0.51 & \pm & 0.07 & 0.52 & \pm & 0.14 & 0.34 & \pm & 0.06 & 0.41 & \pm & 0.07 & 0.47 & \pm & 0.11 \\
\hline Tumor & 4.35 & \pm 1 & 0.25 & 6.05 & \pm & 0.63 & 7.42 & \pm & 0.69 & 10.6 & \pm & 2.26 & 9.09 & \pm & 1.69 & 13.9 & \pm & 1.37 & 13.9 & \pm & 2.17 \\
\hline \multicolumn{22}{|c|}{ Tumor-to-tissue ratios (rel. u.) } \\
\hline Tumor/Blood & 0.124 & \pm( & 0.011 & 0.19 & \pm & 0.02 & 0.33 & \pm & 0.05 & 0.51 & \pm & 0.12 & 0.59 & \pm & 0.13 & 0.85 & \pm & 0.12 & 0.86 & \pm & 0.17 \\
\hline Tumor/Liver & 0.35 & \pm( & 0.02 & 0.47 & \pm & 0.05 & 0.78 & \pm & 0.14 & 1.2 & \pm & 0.3 & 1.1 & \pm & 0.3 & 1.6 & \pm & 0.2 & 1.6 & \pm & 0.3 \\
\hline Tumor/S. I. & 1.51 & \pm( & 0.14 & 1.9 & \pm & 0.2 & 3.1 & \pm & 0.4 & 5.1 & \pm & 1.3 & 5.1 & \pm & 1.2 & 7.8 & \pm & 0.9 & 6.3 & \pm & 1.3 \\
\hline Tumor/L. I. & 2.5 & \pm( & 0.3 & 2.8 & \pm & 0.3 & 3.6 & \pm & 0.5 & 5.1 & \pm & 1.9 & 4.4 & \pm & 1.2 & 6.8 & \pm & 1.5 & 9 & \pm & 2 \\
\hline Tumor/Kidney & 0.23 & 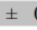 & 0.02 & 0.23 & \pm & 0.03 & 0.22 & \pm & 0.03 & 0.31 & \pm & 0.09 & 0.22 & \pm & 0.04 & 0.40 & \pm & 0.04 & 0.26 & \pm & 0.05 \\
\hline Tumor/Muscle & 4.3 & \pm & 0.7 & 4.9 & \pm & 0.6 & 6.9 & \pm & 1.1 & 6.9 & \pm & 1.9 & 9 & \pm & 4 & 9.5 & \pm & 1.4 & 9 & \pm & 2 \\
\hline Tumor/Bone & 2.0 & \pm & 0.2 & 3.7 & \pm & 0.8 & 4.1 & \pm & 1.3 & 6 & \pm & 3 & 6.3 & \pm & 1.4 & 6.0 & \pm & 0.7 & 5.9 & & 1.0 \\
\hline
\end{tabular}

Table S12-2. Tissue uptake (mean $\% \mathrm{ID} / \mathrm{g} \pm \mathrm{SD}$ ) of ${ }^{18} \mathrm{~F}-\mathrm{AlF}-\mathrm{NO} 2 \mathrm{~A}$-cysVar3 administered via the lateral tail vein in female, BALB/c mice with orthotopic 4T1 breast cancer allografts implanted into the mammary fat pad. 


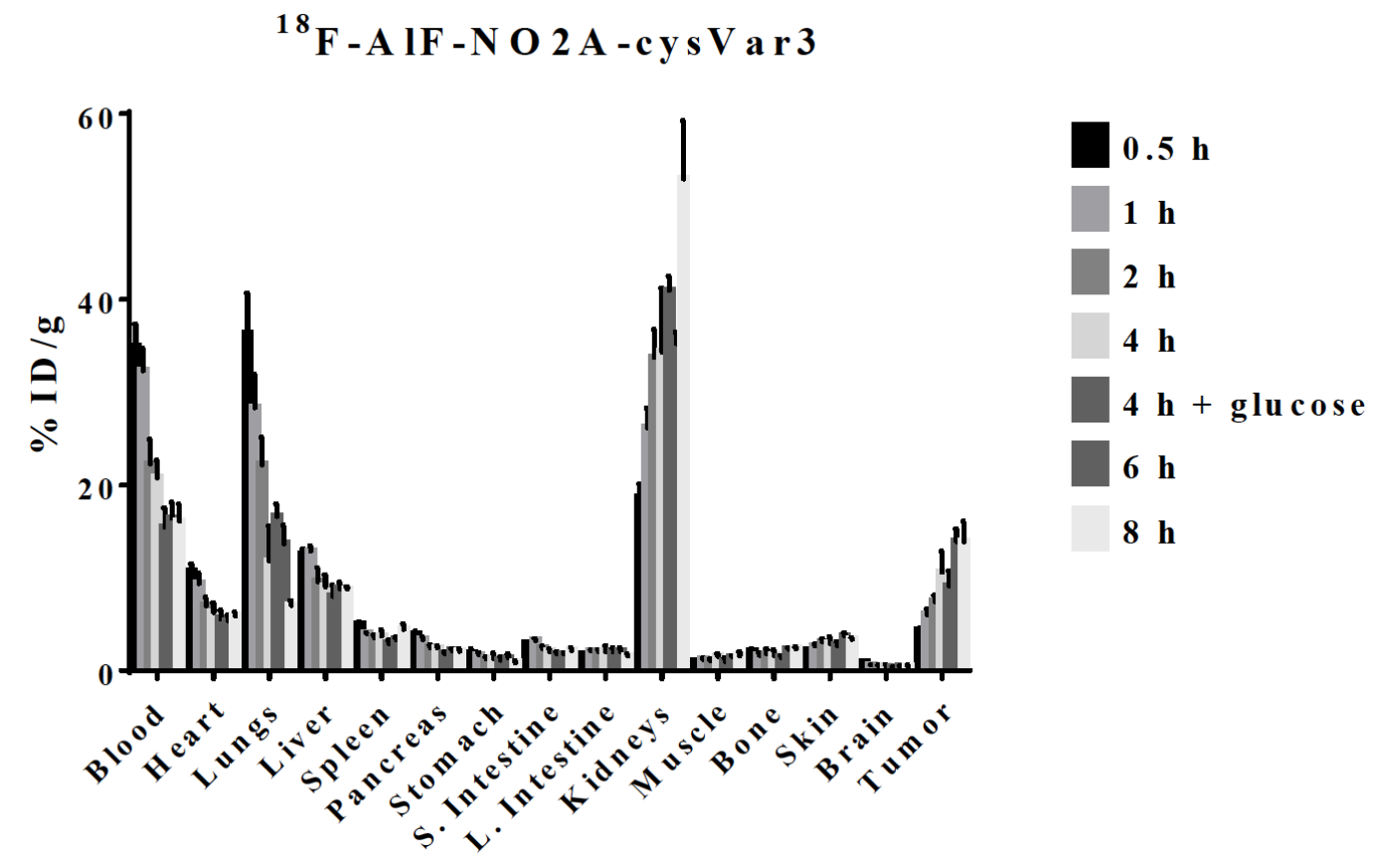

Fig. S12-1. Tissue uptake (mean \%ID/g \pm SD) of ${ }^{18}$ F-AlF-NO2A-cysVar3 (n = 4-10) administered via the lateral tail vein in female, BALB/c mice with orthotopic 4T1 breast cancer allografts implanted into the mammary fat pad full graph from text. Glucose was administered i.p. 30 min prior to tracer injection. 


\section{BIOSCAN}

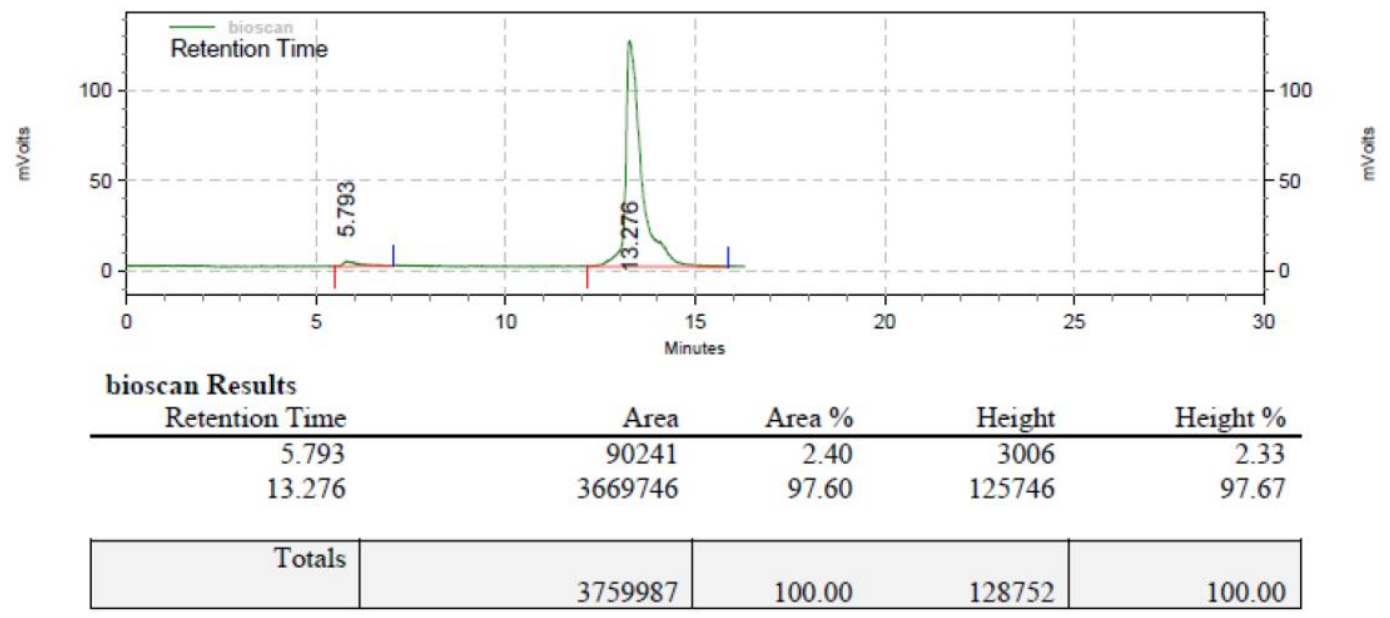

Fig. S12-2. HPLC Chromatogram of the Reconstituted ${ }^{18}$ F-AlF-NO2A-cysVar3 Injectate 


\section{DATA FROM LEAD TRACERS IN OTHER TUMOR MODELS}

\section{${ }^{64} \mathrm{Cu}-\mathrm{NO2A}$-cysVar3 in PC3 tumor-bearing male nude mice}

\begin{tabular}{|c|c|c|c|c|c|c|c|c|c|c|c|c|c|c|c|}
\hline \multirow{2}{*}{$\begin{array}{l}\text { Tissue } \\
\text { Blood }\end{array}$} & \multicolumn{3}{|c|}{$1 \mathrm{~h}(\mathrm{n}=8)$} & \multicolumn{3}{|c|}{$4 h(n=8)$} & \multicolumn{3}{|c|}{$12 \mathrm{~h}(\mathrm{n}=8)$} & \multicolumn{3}{|c|}{$24 \mathrm{~h}(\mathrm{n}=8)$} & \multicolumn{3}{|c|}{$48 \mathrm{~h}(\mathrm{n}=8)$} \\
\hline & 18.3 & \pm & 3.05 & 10.1 & 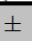 & 3.52 & 3.98 & \pm & 1.12 & 2.30 & \pm & 0.82 & 0.55 & \pm & 0.21 \\
\hline Heart & 0.80 & \pm & 0.12 & 0.81 & $\perp$ & 0.28 & 66 & \pm & 0.10 & 49 & \pm & 0.08 & 0.41 & \pm & 0.04 \\
\hline Lungs & 2.09 & \pm & 0.34 & 1.68 & \pm & 0.36 & 1.29 & \pm & 0.44 & 1.20 & \pm & 0.18 & 0.90 & \pm & 0.10 \\
\hline Liver & 10.1 & + & 1.67 & 11.6 & & 0.97 & 14.0 & \pm & 2.35 & 16.7 & \pm & 3.53 & 12.9 & \pm & 1.90 \\
\hline Spleen & 0.33 & & 0.05 & 0.33 & & 0.04 & 0.26 & \pm & 0.04 & 0.29 & \pm & 0.07 & 0.22 & \pm & 0.03 \\
\hline Pancreas & 0.37 & \pm & 0.05 & 0.30 & $\perp$ & 0.03 & 0.27 & \pm & 0.02 & 0.31 & \pm & 0.04 & 0.25 & \pm & 0.05 \\
\hline Stomach & 0.56 & \pm & 0.12 & 0.72 & \pm & 0.06 & 0.93 & \pm & 0.17 & 1.09 & \pm & 0.29 & 1.12 & \pm & 0.26 \\
\hline S. intestine & 3.20 & 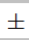 & 1.01 & 3.69 & $=$ & 0.28 & 4.98 & \pm & 0.75 & 6.06 & \pm & 0.90 & 4.52 & \pm & 0.67 \\
\hline L. in & 1.38 & & 0.24 & 2.43 & \pm & 0.66 & 3.96 & \pm & 0.31 & 7.69 & \pm & 1.23 & 7.83 & \pm & 1.28 \\
\hline Kidneys & 7.74 & & 2.26 & 8.61 & & 1.42 & 7.29 & \pm & 0.98 & 5.43 & \pm & 0.62 & 4.05 & \pm & 0.48 \\
\hline Muscle & 0.16 & $\perp$ & 0.03 & 0.15 & \pm & 0.02 & 0.13 & \pm & 0.04 & 0.12 & \pm & 0.03 & 0.10 & & 0.03 \\
\hline Bone & 0.07 & $E$ & 0.02 & 0.10 & \pm & 0.07 & 0.10 & \pm & 0.01 & 0.10 & \pm & 0.04 & 0.08 & \pm & 0.01 \\
\hline Skin & 0.68 & \pm & 0.37 & 0.85 & \pm & 0.28 & 0.63 & \pm & 0.20 & 0.66 & \pm & 0.22 & 0.42 & \pm & 0.11 \\
\hline Tumor & 0.78 & $E$ & 0.30 & 1.04 & \pm & 0.49 & 1.55 & \pm & 0.71 & 2.34 & \pm & 0.86 & 2.42 & \pm & 0.78 \\
\hline
\end{tabular}

Table S13-1. Tissue uptake (mean $\% \mathrm{ID} \pm \mathrm{SD}$ ) of ${ }^{64} \mathrm{Cu}-\mathrm{NO} 2 \mathrm{~A}-\mathrm{cys} V$ ar3 administered via the lateral tail vein in male, nude mice with shoulder PC3 (prostate cancer) xenografts. 


\begin{tabular}{|c|c|c|c|c|c|c|c|c|c|c|c|c|c|c|}
\hline \multirow{2}{*}{$\begin{array}{l}\text { Tissue } \\
\text { Blood }\end{array}$} & \multicolumn{2}{|c|}{$1 \mathrm{~h}(\mathrm{n}=8)$} & \multicolumn{3}{|c|}{$4 h(n=8)$} & \multicolumn{3}{|c|}{$12 \mathrm{~h}(\mathrm{n}=8)$} & \multicolumn{3}{|c|}{$24 \mathrm{~h}(\mathrm{n}=8)$} & \multicolumn{3}{|c|}{$48 h(n=8)$} \\
\hline & 22.0 & \pm 2.88 & 15.6 & \pm & 2.21 & 7.51 & \pm & 1.77 & 5.12 & \pm & 0.77 & 2.48 & \pm & 0.24 \\
\hline Heart & 6.50 & \pm 0.80 & 5.71 & \pm & 1.21 & 3.79 & \pm & 0.82 & 3.32 & \pm & 0.46 & 3.44 & \pm & 0.26 \\
\hline Lungs & 10.7 & \pm 1.66 & 8.63 & \pm & 1.01 & 5.85 & \pm & 0.89 & 5.69 & \pm & 0.51 & 4.37 & \pm & 0.57 \\
\hline Liver & 8.38 & \pm 1.34 & 9.74 & \pm & 1.10 & 9.66 & \pm & 1.35 & 12.4 & \pm & 2.39 & 11.1 & \pm & 1.84 \\
\hline Spleen & 3.42 & \pm 0.25 & 3.17 & \pm & 0.27 & 2.96 & \pm & 0.54 & 3.39 & \pm & 0.25 & 3.06 & \pm & 0.31 \\
\hline Pancreas & 2.38 & \pm 0.40 & 2.02 & \pm & 0.19 & 1.68 & \pm & 0.31 & 1.74 & \pm & 0.17 & 1.62 & \pm & 0.21 \\
\hline Stomach & 1.46 & \pm 0.44 & 1.41 & \pm & 0.50 & 1.30 & \pm & 0.39 & 1.56 & \pm & 0.41 & 2.18 & \pm & 0.35 \\
\hline S. intestine & 2.02 & \pm 0.55 & 2.34 & \pm & 0.38 & 2.22 & \pm & 0.29 & 2.94 & \pm & 0.36 & 3.08 & \pm & 0.17 \\
\hline L. intestine & 0.72 & \pm 0.17 & 1.34 & \pm & 0.48 & 1.80 & \pm & 0.23 & 3.13 & \pm & 0.48 & 3.26 & \pm & 0.18 \\
\hline Kidneys & 16.1 & \pm 4.73 & 17.5 & \pm & 2.72 & 13.9 & \pm & 1.20 & 10.9 & \pm & 1.11 & 8.88 & \pm & 0.68 \\
\hline Muscle & 1.13 & \pm 0.15 & 1.21 & \pm & 0.11 & 1.05 & \pm & 0.20 & 0.90 & \pm & 0.09 & 0.81 & \pm & 0.15 \\
\hline Bone & 1.86 & \pm 0.31 & 1.71 & \pm & 0.26 & 1.54 & \pm & 0.26 & 1.61 & \pm & 0.16 & 1.41 & & 0.15 \\
\hline Skin & 2.80 & \pm 0.73 & 4.39 & \pm & 0.84 & 3.67 & \pm & 0.69 & 3.37 & \pm & 0.37 & 2.60 & \pm & 0.42 \\
\hline Tumor & 3.16 & \pm 0.49 & 4.61 & \pm & 0.82 & 5.58 & \pm & 1.12 & 6.60 & \pm & 0.76 & 6.32 & \pm & 0.81 \\
\hline \multicolumn{15}{|c|}{ Tumor-to-tissue ratios (rel. u.) } \\
\hline Tumor/Blood & 0.14 & \pm 0.03 & 0.29 & \pm & 0.07 & 0.7 & \pm & 0.2 & 1.3 & \pm & 0.2 & 2.6 & \pm & 0.4 \\
\hline Tumor/Liver & 0.38 & \pm 0.08 & 0.47 & \pm & 0.10 & 0.58 & \pm & 0.14 & 0.53 & \pm & 0.12 & 0.57 & \pm & 0.12 \\
\hline Tumor/S. I. & 1.6 & \pm 0.5 & 2.0 & \pm & 0.5 & 2.5 & \pm & 0.6 & 2.2 & \pm & 0.4 & 2.1 & & 0.3 \\
\hline Tumor/L. I. & 4.4 & \pm 1.2 & 3.4 & \pm & 1.4 & 3.1 & \pm & 0.7 & 2.1 & \pm & 0.4 & 1.9 & & 0.3 \\
\hline Tumor/Kidney & 0.20 & \pm 0.07 & 0.26 & \pm & 0.06 & 0.40 & \pm & 0.09 & 0.61 & \pm & 0.09 & 0.71 & & 0.11 \\
\hline Tumor/Muscle & 2.8 & \pm 0.6 & 3.8 & \pm & 0.8 & 5.3 & \pm & 1.5 & 7.3 & \pm & 1.1 & 7.8 & & 1.8 \\
\hline Tumor/Bone & 1.7 & \pm 0.4 & 2.7 & \pm & 0.6 & 3.6 & \pm & 0.9 & 4.1 & \pm & 0.6 & 4.5 & \pm & 0.7 \\
\hline
\end{tabular}

Table S13-2. Tissue uptake (mean $\% \mathrm{ID} / \mathrm{g} \pm \mathrm{SD}$ ) of ${ }^{64} \mathrm{Cu}-\mathrm{NO} 2 \mathrm{~A}-\mathrm{cys}$ Var3 administered via the lateral tail vein in male, nude mice with shoulder PC3 (prostate cancer) xenografts. 
${ }^{64} \mathrm{Cu}-\mathrm{NO} 2 \mathrm{~A}-\mathrm{cys} V \mathrm{ar} 3$ in LNCaP tumor-bearing male nude mice

\begin{tabular}{|c|c|c|c|c|c|c|c|c|c|c|c|c|c|c|c|}
\hline Tissue & \multicolumn{3}{|c|}{$1 \mathrm{~h}(\mathrm{n}=8)$} & \multicolumn{3}{|c|}{$4 h(n=8)$} & \multicolumn{3}{|c|}{$12 h(n=8)$} & \multicolumn{3}{|c|}{$24 \mathrm{~h}(\mathrm{n}=8)$} & \multicolumn{3}{|c|}{$48 \mathrm{~h}(\mathrm{n}=11)$} \\
\hline Blood & 12.3 & \pm & 5.56 & 11.1 & \pm & 3.10 & 3.77 & 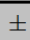 & 1.21 & 4.29 & \pm & 1.48 & 1.42 & \pm & 0.55 \\
\hline Heart & 0.94 & \pm & 0.27 & 0.84 & \pm & 0.29 & 0.56 & & 0.12 & 0.54 & 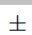 & 0.11 & 0.38 & \pm & 0.06 \\
\hline Lungs & .50 & \pm & 0.60 & 1.94 & \pm & 0.49 & 1.33 & & 0.3 & .25 & \pm & 0.40 & 0.71 & \pm & 0.12 \\
\hline Liver & 10.6 & \pm & 1.85 & 10.1 & \pm & 2.02 & 3.0 & \pm & 2.07 & 4.5 & \pm & .77 & 10.2 & \pm & 3.13 \\
\hline Spleen & .34 & \pm & 0.07 & 0.26 & \pm & 0.04 & 0.30 & \pm & 0.06 & 0.35 & \pm & 0.09 & 0.25 & \pm & 0.09 \\
\hline Pancreas & 0.42 & \pm & 0.05 & 0.32 & \pm & 0.06 & 0.25 & & 0.04 & 0.33 & 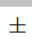 & 0.05 & 0.26 & \pm & 0.05 \\
\hline Ston & 62 & \pm & 0.2 & 0.77 & \pm & 0.1 & 0.86 & & 0.3 & 1.34 & & 0.28 & 0.95 & \pm & 0.26 \\
\hline S. in & 3.29 & \pm & 0.54 & 3.99 & \pm & 1.00 & 4.15 & + & 0.64 & 6.92 & \pm & 1.91 & 4.17 & \pm & 0.55 \\
\hline L. intestir & 1.37 & \pm & 0.30 & 1.94 & \pm & 0.69 & 3.91 & & 0.71 & 7.40 & \pm & 2.22 & 5.95 & \pm & 0.98 \\
\hline Kidneys & 7.67 & \pm & 2.14 & 8.46 & \pm & 2.83 & 7.11 & & 0.65 & 5.94 & \pm & 1.57 & 3.44 & \pm & 0.62 \\
\hline Muscle & 0.16 & \pm & 0.04 & 0.13 & \pm & 0.03 & 0.15 & & $0 .($ & 0.13 & \pm & 0.05 & 0.08 & \pm & 0.02 \\
\hline Bone & 0.07 & \pm & 0.03 & 0.08 & \pm & 0.03 & 0.09 & & 0.02 & 0.08 & \pm & 0.02 & 0.04 & \pm & 0.03 \\
\hline Skin & 0.49 & \pm & 0.17 & 0.78 & \pm & 0.34 & 0.58 & \pm & 0.10 & 0.85 & \pm & 0.31 & 0.41 & \pm & 0.22 \\
\hline Tumor & 0.43 & \pm & 0.48 & 1.13 & \pm & 0.99 & 0.50 & \pm & 0.48 & 2.24 & \pm & 1.05 & 1.53 & \pm & 1.67 \\
\hline
\end{tabular}

Table S13-3. Tissue uptake (mean $\%$ ID $\pm \mathrm{SD}$ ) of ${ }^{64} \mathrm{Cu}-\mathrm{NO} 2 \mathrm{~A}-\mathrm{cys}$ Var3 administered via the lateral tail vein in male, nude mice with shoulder LNCaP (prostate cancer) xenografts. 


\begin{tabular}{|c|c|c|c|c|c|c|c|c|c|c|c|c|c|c|c|}
\hline \multirow{2}{*}{$\frac{\text { Tissue }}{\text { Blood }}$} & \multicolumn{3}{|c|}{$1 \mathrm{~h}(\mathrm{n}=8)$} & \multicolumn{3}{|c|}{$4 h(n=8)$} & \multicolumn{3}{|c|}{$12 h(n=8)$} & \multicolumn{3}{|c|}{$24 \mathrm{~h}(\mathrm{n}=8)$} & \multicolumn{3}{|c|}{$48 \mathrm{~h}(\mathrm{n}=11)$} \\
\hline & 20.8 & \pm & 5.81 & 15.3 & \pm & 3.85 & 6.60 & \pm & 1.47 & 7.21 & \pm & 2.52 & 2.56 & \pm & 0.78 \\
\hline Heart & 6.30 & \pm & 1.68 & 5.49 & \pm & 1.25 & 3.09 & \pm & 0.56 & 4.71 & \pm & 2.50 & 2.94 & \pm & 0.48 \\
\hline Lungs & 11.8 & \pm & 3.01 & 9.27 & \pm & 2.16 & 5.57 & \pm & 0.76 & 7.45 & \pm & 5.42 & 4.17 & \pm & 0.78 \\
\hline Liver & 8.49 & \pm & 2.99 & 8.94 & \pm & 2.51 & 8.22 & \pm & 1.01 & 13.4 & \pm & 3.93 & 8.78 & \pm & 1.16 \\
\hline Spleen & 3.63 & \pm & 1.58 & 2.99 & \pm & 0.79 & 2.65 & \pm & 0.23 & 4.17 & \pm & 1.31 & 3.39 & \pm & 0.87 \\
\hline Pancreas & 2.30 & \pm & 0.55 & 1.86 & \pm & 0.34 & 1.43 & \pm & 0.13 & 1.89 & \pm & 0.36 & 1.54 & \pm & 0.23 \\
\hline Stomach & 1.70 & \pm & 0.81 & 1.32 & \pm & 0.44 & 0.96 & \pm & 0.36 & 1.94 & \pm & 0.64 & 1.61 & \pm & 0.56 \\
\hline S. intestine & 2.07 & \pm & 0.42 & 2.11 & \pm & 0.53 & 1.99 & \pm & 0.37 & 3.08 & \pm & 0.81 & 2.40 & \pm & 0.46 \\
\hline L. intestine & 0.72 & \pm & 0.17 & 0.99 & \pm & 0.38 & 2.11 & \pm & 0.58 & 3.65 & \pm & 0.67 & 3.13 & \pm & 0.66 \\
\hline Kidneys & 14.9 & \pm & 6.43 & 16.7 & \pm & 6.94 & 11.6 & \pm & 0.66 & 12.9 & \pm & 5.07 & 7.49 & \pm & 1.47 \\
\hline Muscle & 1.00 & \pm & 0.31 & 1.07 & \pm & 0.21 & 0.87 & \pm & 0.14 & 1.14 & \pm & 0.49 & 0.74 & \pm & 0.19 \\
\hline Bone & 1.87 & \pm & 1.16 & 1.51 & \pm & 0.33 & 1.32 & \pm & 0.14 & 1.67 & \pm & 0.46 & 1.12 & \pm & 0.27 \\
\hline Skin & 2.33 & \pm & 0.59 & 3.72 & \pm & 0.91 & 3.36 & \pm & 0.52 & 4.32 & \pm & 0.87 & 2.42 & \pm & 0.38 \\
\hline Tumor & 4.09 & \pm & 1.43 & 6.32 & \pm & 2.15 & 5.14 & \pm & 2.65 & 9.47 & \pm & 3.32 & 6.12 & \pm & 1.92 \\
\hline \multicolumn{16}{|c|}{ Tumor-to-tissue ratios (rel. u.) } \\
\hline Tumor/Blood & 0.20 & \pm & 0.09 & 0.41 & \pm & 0.18 & 0.78 & \pm & 0.44 & 1.31 & \pm & 0.65 & 2.40 & \pm & 1.05 \\
\hline Tumor/Liver & 0.48 & \pm & 0.24 & 0.71 & \pm & 0.31 & 0.62 & \pm & 0.33 & 0.71 & \pm & 0.32 & 0.70 & \pm & 0.24 \\
\hline Tumor/S. I. & 1.98 & \pm & 0.80 & 3.00 & \pm & 1.27 & 2.59 & \pm & 1.42 & 3.08 & \pm & 1.35 & 2.55 & \pm & 0.94 \\
\hline Tumor/L. I. & 5.65 & \pm & 2.36 & 6.38 & \pm & 3.27 & 2.43 & \pm & 1.42 & 2.60 & \pm & 1.03 & 1.96 & \pm & 0.74 \\
\hline Tumor/Kidney & 0.28 & \pm & 0.15 & 0.38 & \pm & 0.20 & 0.44 & \pm & 0.23 & 0.74 & \pm & 0.39 & 0.82 & \pm & 0.30 \\
\hline Tumor/Muscle & 4.08 & \pm & 1.91 & 5.89 & \pm & 2.30 & 5.92 & \pm & 3.21 & 8.32 & \pm & 4.60 & 8.28 & \pm & 3.33 \\
\hline Tumor/Bone & 2.19 & \pm & 1.56 & 4.19 & \pm & 1.70 & 3.90 & \pm & 2.05 & 5.68 & \pm & 2.52 & 5.48 & \pm & 2.17 \\
\hline
\end{tabular}

Table S13-4. Tissue uptake (mean $\% \mathrm{ID} / \mathrm{g} \pm \mathrm{SD}$ ) of ${ }^{64} \mathrm{Cu}-\mathrm{NO} 2 \mathrm{~A}-\mathrm{cys}$ Var3 administered via the lateral tail vein in male, nude mice with shoulder LNCaP (prostate cancer) xenografts. 
${ }^{64} \mathrm{Cu}-\mathrm{NO2A}$-cysVar3 in B16-F10 tumor-bearing female C57B1/6 mice

\begin{tabular}{|c|c|c|c|c|c|c|c|c|c|c|c|c|c|c|c|}
\hline Tissue & \multicolumn{3}{|c|}{$1 \mathrm{~h}(\mathrm{n}=4)$} & \multicolumn{3}{|c|}{$4 h(n=3)$} & \multicolumn{3}{|c|}{$12 \mathrm{~h}(\mathrm{n}=4)$} & \multicolumn{3}{|c|}{$14.5 \mathrm{~h}(\mathrm{n}=3)$} & \multicolumn{3}{|c|}{$24 h(n=4)$} \\
\hline Blood & 18.6 & \pm & 1.60 & 6.70 & \pm & 1.64 & 3.95 & \pm & 0.65 & 1.05 & \pm & 0.36 & 1.37 & \pm & 0.67 \\
\hline Heart & 1.28 & \pm & 0.67 & 0.76 & \pm & 0.07 & 0.58 & \pm & 0.08 & 0.63 & \pm & 0.13 & 0.45 & \pm & 0.03 \\
\hline Lungs & 2.55 & \pm & 0.25 & 2.26 & \pm & 0.43 & 2.58 & \pm & 0.53 & 1.93 & \pm & 0.03 & 1.09 & \pm & 0.17 \\
\hline Liver & 9.24 & \pm & 1.45 & 9.54 & \pm & 0.50 & 11.1 & \pm & 1.04 & 9.77 & \pm & 0.63 & 11.3 & \pm & 0.74 \\
\hline Spleen & 0.37 & \pm & 0.09 & 0.32 & \pm & 0.04 & 0.34 & \pm & 0.02 & 0.37 & \pm & 0.09 & 0.35 & \pm & 0.05 \\
\hline Stomach & 0.77 & \pm & 0.20 & 0.76 & \pm & 0.13 & 1.36 & \pm & 0.95 & 0.78 & \pm & 0.17 & 1.27 & \pm & 0.17 \\
\hline S. intestine & 4.38 & \pm & 0.94 & 3.94 & & 0.90 & 5.64 & \pm & 0.68 & 4.52 & \pm & 0.66 & 5.16 & \pm & 0.36 \\
\hline L. intestine & 1.35 & \pm & 0.33 & 2.72 & & 0.42 & 3.44 & \pm & 0.38 & 3.89 & \pm & 0.25 & 6.11 & \pm & 0.27 \\
\hline Kidneys & 4.54 & \pm & 0.78 & 8.92 & \pm & 0.99 & 8.08 & \pm & 0.88 & 6.61 & \pm & 1.23 & 4.82 & \pm & 0.43 \\
\hline Muscle & 0.10 & \pm & 0.03 & 0.15 & \pm & 0.05 & 0.15 & \pm & 0.03 & 0.18 & \pm & 0.07 & 0.13 & \pm & 0.05 \\
\hline Bone & 0.07 & \pm & 0.02 & 0.17 & \pm & 0.02 & 0.13 & \pm & 0.04 & 0.35 & \pm & 0.05 & 0.21 & \pm & 0.08 \\
\hline Skin (ears) & 0.30 & \pm & 0.19 & 0.33 & \pm & 0.08 & 0.28 & \pm & 0.10 & 0.14 & \pm & 0.01 & 0.36 & \pm & 0.10 \\
\hline Skin (shoulder) & 0.26 & \pm & 0.09 & 0.22 & \pm & 0.03 & 0.26 & \pm & 0.10 & 0.21 & \pm & 0.04 & 0.15 & \pm & 0.03 \\
\hline Tumor & 2.15 & \pm & 1.37 & 1.45 & \pm & 2.02 & 3.58 & \pm & 2.10 & 1.27 & \pm & 1.95 & 1.61 & \pm & 0.93 \\
\hline
\end{tabular}

Table S13-5. Tissue uptake (mean \%ID $\pm \mathrm{SD}$ ) of ${ }^{64} \mathrm{Cu}-\mathrm{NO} 2 \mathrm{~A}-\mathrm{cys}$ Var3 administered via the lateral tail vein in female, C57Bl/6 mice with orthotopic B16-F10 (melanoma) allografts. 


\begin{tabular}{|c|c|c|c|c|c|c|c|c|c|c|c|c|c|c|c|}
\hline \multirow{2}{*}{$\begin{array}{l}\text { Tissue } \\
\text { Blood }\end{array}$} & \multicolumn{3}{|c|}{$1 \mathrm{~h}(\mathrm{n}=4)$} & \multicolumn{3}{|c|}{$4 h(n=3)$} & \multicolumn{3}{|c|}{$12 h(n=4)$} & \multicolumn{3}{|c|}{$14.5 \mathrm{~h}(\mathrm{n}=3)$} & \multicolumn{3}{|c|}{$24 h(n=4)$} \\
\hline & 28.4 & \pm & 0.74 & 19.5 & \pm & 1.06 & 11.6 & $\overline{ \pm}$ & 1.52 & 12.0 & \pm & 2.14 & 6.37 & \pm & 0.39 \\
\hline Heart & 8.24 & \pm & 0.28 & 6.86 & \pm & 0.52 & 4.55 & \pm & 0.64 & 4.75 & \pm & 0.72 & 4.32 & \pm & 0.41 \\
\hline Lungs & 12.6 & \pm & 1.32 & 11.8 & \pm & 3.38 & 9.94 & \pm & 1.74 & 8.97 & \pm & 1.30 & 5.81 & \pm & 0.59 \\
\hline Liver & 11.1 & \pm & 1.47 & 10.3 & \pm & 0.38 & 8.97 & \pm & 0.68 & 10.1 & \pm & 1.35 & 11.7 & \pm & 1.42 \\
\hline Spleen & 4.24 & \pm & 0.34 & 4.33 & \pm & 0.10 & 4.22 & \pm & 0.41 & 4.54 & \pm & 0.63 & 4.96 & \pm & 0.29 \\
\hline Stomach & 2.53 & \pm & 0.45 & 1.55 & \pm & 0.07 & 1.55 & \pm & 0.73 & 2.00 & \pm & 0.37 & 3.25 & \pm & 0.54 \\
\hline S. intestine & 3.41 & \pm & 0.30 & 3.14 & \pm & 0.30 & 3.43 & \pm & 0.30 & 4.00 & \pm & 0.29 & 5.44 & \pm & 0.87 \\
\hline L. intestine & 1.94 & \pm & 0.40 & 2.98 & \pm & 0.13 & 3.27 & \pm & 0.37 & 4.35 & \pm & 0.28 & 7.24 & \pm & 0.51 \\
\hline Kidneys & 13.5 & \pm & 2.19 & 28.5 & \pm & 5.17 & 26.0 & \pm & 2.38 & 21.0 & \pm & 1.61 & 16.2 & \pm & 1.31 \\
\hline Muscle & 0.75 & \pm & 0.12 & 1.48 & \pm & 0.31 & 1.23 & \pm & 0.13 & 1.31 & \pm & 0.30 & 1.28 & \pm & 0.28 \\
\hline Bone & 1.86 & \pm & 0.49 & 2.53 & \pm & 0.17 & 2.03 & \pm & 0.39 & 2.66 & \pm & 0.39 & 2.28 & \pm & 0.19 \\
\hline Skin ( & 1.80 & \pm & 0.50 & 3.90 & \pm & 0.71 & 3.35 & \pm & 0.30 & 3.55 & \pm & 0.30 & 3.73 & \pm & 0.56 \\
\hline Skin (sh & 1.98 & & 0.43 & 5.68 & \pm & 0.65 & 3.93 & \pm & 0.73 & 5.49 & \pm & 0.41 & 5.09 & \pm & 0.88 \\
\hline Tumor & 5.42 & \pm & 0.76 & 9.29 & \pm & 0.43 & 11.6 & \pm & 0.51 & 11.2 & \pm & 0.94 & 11.4 & & 2.92 \\
\hline \multicolumn{16}{|c|}{ Tumor-to-tissue ratios (rel. u.) } \\
\hline Tumor/Blood & 0.19 & \pm & 0.03 & 0.48 & \pm & 0.03 & 1.00 & \pm & 0.14 & 0.94 & \pm & 0.19 & 1.8 & & 0.5 \\
\hline Tumor/Liver & 0.49 & \pm & 0.09 & 0.90 & \pm & 0.05 & 1.29 & \pm & 0.11 & 1.11 & \pm & 0.17 & 1.0 & & 0.3 \\
\hline Tumor/S. I. & 1.6 & \pm & 0.3 & 3.0 & \pm & 0.3 & 3.4 & \pm & 0.3 & 2.8 & \pm & 0.3 & 2.1 & & 0.6 \\
\hline Tumor/L. I. & 2.8 & \pm & 0.7 & 3.1 & \pm & 0.2 & 3.5 & \pm & 0.4 & 2.6 & \pm & 0.3 & 1.6 & \pm & 0.4 \\
\hline Tumor/Kidney & 0.40 & \pm & 0.09 & 0.33 & \pm & 0.06 & 0.45 & \pm & 0.05 & 0.53 & \pm & 0.06 & 0.70 & \pm & 0.19 \\
\hline Tumor/Muscle & 7.2 & \pm & 1.5 & 6.3 & \pm & 1.4 & 9.4 & \pm & 1.1 & 9 & \pm & 2 & 9 & & 3 \\
\hline Tumor/Bone & 2.9 & \pm & 0.9 & 3.7 & \pm & 0.3 & 5.7 & \pm & 1.1 & 4.2 & \pm & 0.7 & 5.0 & & 1.3 \\
\hline
\end{tabular}

Table S13-6. Tissue uptake (mean $\% \mathrm{ID} / \mathrm{g} \pm \mathrm{SD}$ ) of ${ }^{64} \mathrm{Cu}-\mathrm{NO} 2 \mathrm{~A}-\mathrm{cys} V \operatorname{Var} 3$ administered via the lateral tail vein in female, C57B1/6 mice with orthotopic B16-F10 (melanoma) allografts. 
${ }^{18}$ F-AIF-NO2A-cysVar3 in PC3 tumor-bearing male nude mice

\begin{tabular}{lcccccccccccc}
\hline Tissue & \multicolumn{3}{c}{$\mathbf{1 ~ h}(\mathbf{n}=\mathbf{3})$} & \multicolumn{3}{c}{$\mathbf{4} \mathbf{~ h}(\mathbf{n}=\mathbf{4})$} & \multicolumn{3}{c}{$\mathbf{6 ~ h}(\mathbf{n}=\mathbf{4})$} & \multicolumn{2}{c}{$\mathbf{8} \mathbf{~ h}(\mathbf{n}=\mathbf{4})$} \\
\hline Blood & 10.7 & \pm & 5.84 & 6.88 & \pm & 1.21 & 6.37 & \pm & 3.17 & 4.62 & \pm & 2.24 \\
Heart & 0.84 & \pm & 0.22 & 0.61 & \pm & 0.07 & 0.82 & \pm 0.19 & 0.68 & \pm & 0.09 \\
Lungs & 2.25 & \pm & 0.84 & 2.06 & \pm & 0.77 & 0.77 & \pm 0.12 & 0.84 & \pm & 0.17 \\
Liver & 9.87 & \pm & 0.55 & 7.09 & \pm & 1.13 & 6.97 & \pm & 1.87 & 6.02 & \pm & 0.51 \\
Spleen & 0.24 & \pm & 0.03 & 0.29 & \pm & 0.06 & 0.21 & \pm 0.05 & 0.35 & \pm & 0.03 \\
Pancreas & 0.37 & \pm & 0.06 & 0.27 & \pm & 0.03 & 0.19 & \pm 0.02 & 0.30 & \pm & 0.09 \\
Stomach & 0.46 & \pm & 0.07 & 0.41 & \pm & 0.09 & 0.35 & \pm 0.04 & 0.42 & \pm & 0.05 \\
S. intestine & 2.66 & \pm & 0.10 & 2.47 & \pm & 0.58 & 1.73 & \pm 0.29 & 2.20 & \pm & 0.22 \\
L. intestine & 1.16 & \pm & 0.06 & 1.32 & \pm & 0.27 & 1.15 & \pm 0.24 & 1.19 & \pm & 0.18 \\
Kidneys & 5.82 & \pm & 0.64 & 10.6 & \pm & 1.97 & 12.3 & \pm & 1.50 & 13.7 & \pm & 0.50 \\
Muscle & 0.10 & \pm & 0.03 & 0.17 & \pm & 0.02 & 0.12 & \pm 0.03 & 0.15 & \pm & 0.01 \\
Bone & 0.05 & \pm & 0.01 & 0.06 & \pm & 0.03 & 0.10 & \pm 0.02 & 0.09 & \pm & 0.01 \\
Skin & 0.59 & \pm & 0.22 & 1.10 & \pm & 0.26 & 0.29 & \pm 0.09 & 0.42 & \pm & 0.09 \\
Tumor & 0.96 & \pm & 0.45 & 1.04 & \pm & 0.55 & 1.11 & \pm 0.53 & 1.79 & \pm & 0.98 \\
\hline
\end{tabular}

Table S13-7. Tissue uptake (mean \%ID \pm SD) of ${ }^{18}$ F-AlF-NO2A-cysVar3 administered via the lateral tail vein in male, nude mice with shoulder PC3 (prostate cancer) xenografts. 


\begin{tabular}{|c|c|c|c|c|c|c|c|c|c|c|c|c|}
\hline \multirow{2}{*}{$\frac{\text { Tissue }}{\text { Blood }}$} & \multicolumn{3}{|c|}{$1 \mathrm{~h}(\mathrm{n}=3)$} & \multicolumn{3}{|c|}{$4 h(n=4)$} & \multicolumn{3}{|c|}{$6 \mathrm{~h}(\mathrm{n}=4)$} & \multicolumn{3}{|c|}{$8 \mathrm{~h}(\mathrm{n}=4)$} \\
\hline & 16.2 & \pm & 2.29 & 11.6 & \pm & 1.83 & 8.66 & \pm & 0.91 & 9.08 & \pm & 0.70 \\
\hline Heart & 5.41 & \pm & 0.99 & 4.04 & \pm & 0.28 & 3.79 & \pm & 1.14 & 4.11 & \pm & 0.14 \\
\hline Lungs & 9.39 & \pm & 3.35 & 7.69 & \pm & 1.72 & 3.93 & \pm & 0.86 & 4.27 & \pm & 0.45 \\
\hline Liver & 6.03 & \pm & 0.68 & 5.12 & \pm & 0.35 & 4.16 & \pm & 0.40 & 4.19 & \pm & 0.45 \\
\hline Spleen & 2.23 & \pm & 0.36 & 2.04 & \pm & 0.11 & 1.83 & \pm & 0.20 & 2.47 & \pm & 0.11 \\
\hline Pancreas & 1.78 & \pm & 0.31 & 1.56 & \pm & 0.20 & 1.13 & \pm & 0.15 & 1.37 & \pm & 0.30 \\
\hline Stomach & 0.65 & \pm & 0.02 & 0.53 & \pm & 0.03 & 0.63 & \pm & 0.10 & 0.44 & \pm & 0.11 \\
\hline S. intestine & 1.28 & \pm & 0.15 & 1.22 & \pm & 0.08 & 0.86 & \pm & 0.14 & 1.38 & \pm & 0.09 \\
\hline L. intestine & 0.58 & \pm & 0.06 & 0.58 & \pm & 0.02 & 0.72 & \pm & 0.19 & 0.74 & \pm & 0.13 \\
\hline Kidneys & 10.7 & \pm & 1.10 & 18.2 & \pm & 3.15 & 19.2 & \pm & 4.25 & 25.8 & \pm & 2.51 \\
\hline Mus & 0.68 & \pm & 0.13 & 1.03 & \pm & 0.11 & 0.90 & \pm & 0.28 & 0.91 & \pm & 0.23 \\
\hline Bone & 1.21 & \pm & 0.29 & 1.24 & \pm & 0.25 & 1.31 & \pm & 0.09 & 1.28 & \pm & 0.26 \\
\hline Skin & 1.96 & \pm & 0.29 & 3.73 & \pm & 0.08 & 2.32 & \pm & 0.46 & 2.53 & \pm & 0.15 \\
\hline Tumor & 3.05 & \pm & 0.36 & 4.02 & \pm & 0.35 & 4.19 & \pm & 0.55 & 3.99 & \pm & 0.27 \\
\hline \multicolumn{13}{|c|}{ Tumor-to-tissue ratios (rel. u.) } \\
\hline Tumor/Blood & 0.19 & \pm & 0.03 & 0.35 & \pm & 0.06 & 0.48 & \pm & 0.08 & 0.44 & & 0.04 \\
\hline Tum & 0.51 & \pm & 0.08 & 0.79 & \pm & 0.09 & 1.01 & \pm & 0.16 & 0.95 & \pm & 0.12 \\
\hline Tum & 2.4 & \pm & 0.4 & 3.3 & \pm & 0.4 & 4.8 & \pm & 1.0 & 2.9 & & 0.3 \\
\hline Tumor/L. & 5.2 & \pm & 0.8 & 7.0 & \pm & 0.7 & 5.8 & \pm & 1.7 & 5.4 & \pm & 1.0 \\
\hline Tumor/ & 0.29 & \pm & 0.04 & 0.22 & \pm & 0.04 & 0.22 & \pm & 0.06 & 0.15 & & 0.02 \\
\hline Tumor/ & 4.5 & \pm & 1.0 & 3.9 & \pm & 0.5 & 4.7 & \pm & 1.6 & 4.4 & \pm & 1.1 \\
\hline Tumor/Bone & 2.5 & \pm & 0.7 & 3.2 & \pm & 0.7 & 3.2 & \pm & 0.5 & 3.1 & \pm & 0.7 \\
\hline
\end{tabular}

Table S13-8. Tissue uptake (mean $\%$ ID/g \pm SD) of ${ }^{18}$ F-AlF-NO2A-cysVar3 administered via the lateral tail vein in male, nude mice with shoulder PC3 (prostate cancer) xenografts. 
${ }^{18}$ F-AIF-NO2A-cysVar3 in LNCaP tumor-bearing male nude mice

\begin{tabular}{|c|c|c|c|c|c|c|c|c|c|c|c|c|}
\hline Tissue & \multicolumn{3}{|c|}{$1 \mathrm{~h}(\mathrm{n}=4)$} & \multicolumn{3}{|c|}{$4 h(n=4)$} & \multicolumn{3}{|c|}{$6 \mathrm{~h}(\mathrm{n}=4)$} & \multicolumn{3}{|c|}{$8 h(n=4)$} \\
\hline Blood & 17.7 & \pm & 4.60 & 11.4 & \pm & 0.78 & 5.43 & \pm & 1.31 & 5.49 & \pm & 1.77 \\
\hline Heart & 0.66 & \pm & 0.12 & 0.62 & \pm & 0.10 & 0.73 & \pm & 0.16 & 0.53 & \pm & 0.08 \\
\hline Lungs & 1.98 & \pm & 0.24 & 1.15 & \pm & 0.14 & 1.33 & \pm & 0.30 & 0.83 & \pm & 0.08 \\
\hline Liver & 7.33 & \pm & 1.08 & 5.00 & \pm & 0.70 & 5.84 & \pm & 0.23 & 5.42 & \pm & 0.28 \\
\hline Spleen & 0.30 & \pm & 0.08 & 0.25 & \pm & 0.03 & 0.25 & \pm & 0.02 & 0.27 & \pm & 0.08 \\
\hline Pancreas & 0.33 & \pm & 0.09 & 0.25 & \pm & 0.05 & 0.23 & \pm & 0.03 & 0.20 & \pm & 0.04 \\
\hline Stomach & 0.47 & \pm & 0.08 & 0.53 & \pm & 0.32 & 0.47 & \pm & 0.03 & 0.43 & \pm & 0.09 \\
\hline S. intestine & 2.16 & \pm & 0.31 & 1.98 & \pm & 0.36 & 1.72 & \pm & 0.08 & 2.57 & \pm & 0.29 \\
\hline L. intestine & 0.97 & \pm & 0.23 & 1.08 & \pm & 0.18 & 1.19 & \pm & 0.09 & 1.38 & \pm & 0.36 \\
\hline Kidneys & 5.61 & \pm & 0.62 & 8.87 & \pm & 1.78 & 11.0 & \pm & 1.50 & 13.9 & \pm & 0.79 \\
\hline Muscle & 0.14 & \pm & 0.03 & 0.16 & \pm & 0.03 & 0.16 & \pm & 0.05 & 0.13 & \pm & 0.02 \\
\hline Bone & 0.05 & \pm & 0.02 & 0.05 & \pm & 0.03 & 0.08 & \pm & 0.01 & 0.08 & \pm & 0.03 \\
\hline Skin & 0.71 & \pm & 0.27 & 0.89 & \pm & 0.17 & 0.42 & \pm & 0.08 & 0.37 & \pm & 0.05 \\
\hline Tumor & 0.39 & \pm & 0.26 & 0.78 & \pm & 0.47 & 3.73 & \pm & 3.95 & 1.81 & \pm & 2.24 \\
\hline
\end{tabular}

Table S13-9. Tissue uptake (mean \%ID \pm SD) of ${ }^{18}$ F-AlF-NO2A-cysVar3 administered via the lateral tail vein in male, nude mice with shoulder LNCaP (prostate cancer) xenografts. 


\begin{tabular}{|c|c|c|c|c|c|c|c|c|c|c|c|c|}
\hline \multirow{2}{*}{$\begin{array}{l}\text { Tissue } \\
\text { Blood }\end{array}$} & \multicolumn{3}{|c|}{$1 \mathrm{~h}(\mathrm{n}=4)$} & \multicolumn{3}{|c|}{$4 \mathrm{~h}(\mathrm{n}=4)$} & \multicolumn{3}{|c|}{$6 h(n=4)$} & \multicolumn{3}{|c|}{$8 h(n=4)$} \\
\hline & 20.0 & \pm & 2.48 & 12.4 & \pm & 1.74 & 12.1 & \pm & 2.16 & 10.2 & \pm & 2.15 \\
\hline Heart & 5.28 & \pm & 0.92 & 4.41 & \pm & 1.11 & 4.39 & \pm & 0.69 & 3.69 & \pm & 0.28 \\
\hline Lungs & 9.19 & \pm & 1.18 & 5.82 & \pm & 0.49 & 6.48 & \pm & 1.05 & 4.42 & \pm & 0.51 \\
\hline Liver & 5.87 & \pm & 0.39 & 4.62 & \pm & 0.51 & 4.56 & \pm & 0.43 & 4.33 & \pm & 0.49 \\
\hline Spleen & 2.79 & \pm & 0.52 & 1.96 & \pm & 0.14 & 1.93 & \pm & 0.25 & 2.60 & \pm & 0.27 \\
\hline Pancreas & 2.01 & \pm & 0.30 & 1.37 & \pm & 0.26 & 1.42 & \pm & 0.24 & 1.42 & \pm & 0.36 \\
\hline Stomach & 1.07 & \pm & 0.21 & 0.70 & \pm & 0.14 & 0.63 & \pm & 0.20 & 0.67 & \pm & 0.13 \\
\hline S. intestine & 1.38 & \pm & 0.26 & 1.03 & \pm & 0.05 & 0.97 & \pm & 0.05 & 1.32 & \pm & 0.29 \\
\hline L. intestine & 0.47 & \pm & 0.14 & 0.42 & \pm & 0.06 & 0.47 & \pm & 0.03 & 0.68 & \pm & 0.34 \\
\hline Kidneys & 10.7 & \pm & 0.68 & 17.3 & \pm & 1.51 & 23.9 & \pm & 3.74 & 28.6 & \pm & 2.36 \\
\hline Muscle & 0.75 & \pm & 0.09 & 0.93 & \pm & 0.16 & 1.14 & \pm & 0.24 & 1.06 & \pm & 0.33 \\
\hline Bone & 1.23 & \pm & 0.22 & 1.02 & \pm & 0.25 & 1.33 & \pm & 0.14 & 1.24 & & 0.27 \\
\hline Skin & 2.59 & \pm & 0.45 & 3.03 & \pm & 0.42 & 2.91 & \pm & 0.48 & 2.64 & \pm & 0.18 \\
\hline Tumor & 3.04 & \pm & 0.37 & 4.41 & \pm & 0.47 & 4.79 & \pm & 1.08 & 4.57 & \pm & 0.75 \\
\hline \multicolumn{13}{|c|}{ Tumor-to-tissue ratios (rel. u.) } \\
\hline Tumor/Blood & 0.15 & \pm & 0.03 & 0.36 & \pm & 0.06 & 0.40 & \pm & 0.11 & 0.45 & \pm & 0.12 \\
\hline Tumor/Liver & 0.52 & \pm & 0.07 & 0.95 & \pm & 0.15 & 1.1 & \pm & 0.3 & 1.1 & \pm & 0.2 \\
\hline Tumor/S. I. & 2.2 & \pm & 0.5 & 4.3 & \pm & 0.5 & 4.9 & \pm & 1.1 & 3.5 & & 0.9 \\
\hline Tumor/L. I. & 7 & \pm & 2 & 10.5 & \pm & 1.8 & 10 & \pm & 2 & 6.7 & & 3.6 \\
\hline Tumor/Kidney & 0.28 & \pm & 0.04 & 0.25 & \pm & 0.03 & 0.20 & \pm & 0.06 & 0.16 & & 0.03 \\
\hline Tumor/Muscle & 4.1 & \pm & 0.7 & 4.7 & \pm & 0.9 & 4.2 & \pm & 1.3 & 4.3 & & 1.5 \\
\hline Tumor/Bone & 2.5 & \pm & 0.5 & 4.3 & \pm & 1.1 & 3.6 & \pm & 0.9 & 3.7 & & \\
\hline
\end{tabular}

Table S13-10. Tissue uptake (mean $\% \mathrm{ID} / \mathrm{g} \pm \mathrm{SD}$ ) of ${ }^{18} \mathrm{~F}$-AlF-NO2A-cysVar3 administered via the lateral tail vein in male, nude mice with shoulder LNCaP (prostate cancer) xenografts. 
${ }^{18}$ F-AIF-NO2A-cysVar3 in B16-F10 tumor-bearing female C57Bl/6 mice

\begin{tabular}{|c|c|c|c|c|c|c|c|c|c|c|c|c|c|c|c|}
\hline \multirow{2}{*}{$\begin{array}{l}\text { Tissue } \\
\text { Blood }\end{array}$} & \multicolumn{3}{|c|}{$1 \mathrm{~h}(\mathrm{n}=4)$} & \multicolumn{3}{|c|}{$4 h(n=3)$} & \multicolumn{3}{|c|}{$6 \mathrm{~h}(\mathrm{n}=4)$} & \multicolumn{3}{|c|}{$8 h(n=3)$} & \multicolumn{3}{|c|}{$6 \mathrm{~h}(\mathrm{n}=3)^{*}$} \\
\hline & 12.7 & \pm & 4.93 & 8.16 & \pm & 1.53 & 4.17 & \pm & 0.88 & 4.17 & \pm & 2.37 & 6.25 & \pm & 2.32 \\
\hline Heart & 0.92 & \pm & 0.13 & 0.58 & \pm & 0.07 & 0.55 & \pm & 0.13 & 0.54 & \pm & 0.08 & 0.67 & \pm & 0.14 \\
\hline Lungs & 2.70 & \pm & 0.89 & 1.80 & \pm & 0.65 & 2.06 & \pm & 0.64 & 1.00 & \pm & 0.10 & 1.53 & \pm & 0.07 \\
\hline Liver & 10.2 & \pm & 0.90 & 6.59 & \pm & 1.19 & 5.94 & \pm & 0.45 & 5.88 & \pm & 0.38 & 6.65 & \pm & 0.57 \\
\hline Spleen & 0.30 & \pm & 0.02 & 0.29 & \pm & 0.07 & 0.28 & \pm & 0.03 & 0.26 & \pm & 0.05 & 0.51 & \pm & 0.08 \\
\hline Stomach & 0.63 & \pm & 0.12 & 0.51 & \pm & 0.05 & 0.42 & \pm & 0.06 & 0.45 & \pm & 0.08 & 0.62 & \pm & 0.09 \\
\hline S. intestine & 3.04 & \pm & 0.45 & 2.20 & \pm & 0.39 & 2.40 & \pm & 0.29 & 2.14 & \pm & 0.19 & 2.79 & \pm & 0.23 \\
\hline L. intestine & 1.04 & \pm & 0.13 & 1.14 & \pm & 0.10 & 1.23 & \pm & 0.05 & 1.28 & \pm & 0.36 & 1.72 & \pm & 0.04 \\
\hline Kidneys & 6.11 & \pm & 0.70 & 8.10 & \pm & 0.58 & 11.8 & \pm & 0.73 & 13.0 & \pm & 1.76 & 10.20 & \pm & 0.11 \\
\hline Muscle & 0.14 & \pm & 0.04 & 0.18 & \pm & 0.06 & 0.15 & \pm & 0.05 & 0.17 & \pm & 0.03 & 0.17 & \pm & 0.04 \\
\hline Bone & 0.07 & \pm & 0.03 & 0.09 & \pm & 0.03 & 0.12 & \pm & 0.04 & 0.14 & \pm & 0.04 & 0.12 & \pm & 0.00 \\
\hline Skin (ears) & 0.33 & \pm & 0.09 & 0.44 & \pm & 0.15 & 0.34 & \pm & 0.06 & 0.24 & \pm & 0.05 & 0.26 & \pm & 0.04 \\
\hline Skin (shoulder) & 0.38 & \pm & 0.11 & 0.58 & \pm & 0.21 & 0.32 & \pm & 0.17 & 0.20 & \pm & 0.08 & 0.16 & \pm & 0.05 \\
\hline Tumor & 0.67 & \pm & 0.16 & 1.56 & \pm & 1.89 & 2.29 & \pm & 1.97 & 1.96 & \pm & 2.18 & 0.11 & \pm & 0.07 \\
\hline
\end{tabular}

Table S13-11. Tissue uptake (mean \%ID \pm SD) of ${ }^{18}$ F-AlF-NO2A-cysVar3 administered via the lateral tail vein in female, C57Bl/6 mice with orthotopic B16-F10 (melanoma) allografts and one group of female, BALB/c mice with orthotopic B16F10 (melanoma) allografts at $6 \mathrm{~h}$. *BALB/c time point. 


\begin{tabular}{|c|c|c|c|c|c|c|c|c|c|c|c|c|c|c|c|}
\hline \multirow{2}{*}{$\begin{array}{l}\text { Tissue } \\
\text { Blood }\end{array}$} & \multicolumn{3}{|c|}{$1 \mathrm{~h}(\mathrm{n}=5)$} & \multicolumn{3}{|c|}{$4 h(n=5)$} & \multicolumn{3}{|c|}{$6 \mathrm{~h}(\mathrm{n}=5)$} & \multicolumn{3}{|c|}{$8 \mathrm{~h}(\mathrm{n}=5)$} & \multicolumn{3}{|c|}{$6 \mathrm{~h}(\mathrm{n}=3)^{*}$} \\
\hline & 29.3 & \pm & 3.06 & 17.2 & \pm & 1.77 & 15.3 & \pm & 0.70 & 14.3 & \pm & 0.80 & 21.1 & \pm & 0.27 \\
\hline Heart & 8.86 & \pm & 1.02 & 5.79 & \pm & 0.63 & 4.86 & \pm & 0.53 & 5.36 & \pm & 0.66 & 6.98 & \pm & 0.81 \\
\hline Lungs & 15.2 & \pm & 1.14 & 10.1 & \pm & 3.12 & 9.70 & \pm & 1.84 & 5.82 & \pm & 0.57 & 8.76 & \pm & 0.47 \\
\hline Liver & 10.1 & \pm & 1.60 & 7.22 & \pm & 0.64 & 7.41 & \pm & 0.20 & 7.01 & \pm & 0.35 & 8.02 & \pm & 0.11 \\
\hline Spleen & 3.79 & \pm & 0.36 & 3.18 & \pm & 0.41 & 3.22 & \pm & 0.19 & 4.00 & \pm & 0.14 & 4.89 & \pm & 0.28 \\
\hline Stomach & 1.90 & \pm & 0.91 & 1.70 & \pm & 0.35 & 1.18 & \pm & 0.27 & 0.82 & \pm & 0.12 & 0.86 & \pm & 0.05 \\
\hline S. intestine & 2.77 & \pm & 0.52 & 2.08 & \pm & 0.28 & 1.89 & \pm & 0.16 & 1.93 & \pm & 0.05 & 2.73 & \pm & 0.09 \\
\hline L. intestine & 1.48 & \pm & 0.30 & 1.84 & \pm & 0.14 & 1.59 & \pm & 0.12 & 1.72 & \pm & 0.23 & 2.05 & \pm & 0.08 \\
\hline Kidneys & 20.1 & \pm & 3.35 & 28.9 & \pm & 3.19 & 40.7 & \pm & 3.41 & 46.0 & \pm & 2.98 & 34.4 & \pm & 0.71 \\
\hline Muscle & 1.09 & \pm & 0.15 & 1.46 & \pm & 0.14 & 1.51 & \pm & 0.23 & 1.46 & \pm & 0.12 & 1.52 & \pm & 0.16 \\
\hline Bone & 2.33 & \pm & 0.34 & 2.22 & \pm & 0.50 & 2.64 & \pm & 0.45 & 2.42 & \pm & 0.13 & 2.75 & \pm & 0.20 \\
\hline Skin (ears) & 2.35 & \pm & 0.34 & 3.61 & \pm & 0.61 & 3.91 & \pm & 0.21 & 2.78 & \pm & 0.17 & 3.00 & \pm & 0.28 \\
\hline Skin (shoulder) & 2.77 & \pm & 0.57 & 4.86 & \pm & 0.74 & 4.93 & \pm & 0.68 & 4.77 & \pm & 0.35 & 5.24 & \pm & 0.44 \\
\hline Tumor & 6.12 & \pm & 0.60 & 8.23 & \pm & 0.84 & 8.02 & \pm & 2.00 & 10.5 & \pm & 1.73 & 8.62 & \pm & 1.53 \\
\hline \multicolumn{16}{|c|}{ Tumor-to-tissue ratios (rel. u.) } \\
\hline Tumor/Blood & 0.21 & \pm & 0.03 & 0.48 & \pm & 0.07 & 0.52 & \pm & 0.13 & 0.73 & \pm & 0.13 & 0.41 & \pm & 0.07 \\
\hline Tumor/Liver & 0.61 & \pm & 0.11 & 1.14 & \pm & 0.15 & 1.1 & \pm & 0.3 & 1.5 & \pm & 0.3 & 1.07 & \pm & 0.19 \\
\hline Tumor/S. I. & 2.2 & \pm & 0.5 & 4.0 & \pm & 0.7 & 4.2 & \pm & 1.1 & 5.4 & \pm & 0.9 & 3.2 & \pm & 0.6 \\
\hline Tumor/L. I. & 4.1 & \pm & 0.9 & 4.5 & \pm & 0.6 & 5.0 & \pm & 1.3 & 6.1 & \pm & 1.3 & 4.2 & \pm & 0.8 \\
\hline Tumor/Kidney & 0.30 & \pm & 0.06 & 0.28 & \pm & 0.04 & 0.20 & \pm & 0.05 & 0.23 & \pm & 0.04 & 0.25 & \pm & 0.04 \\
\hline Tumor/Muscle & 5.6 & \pm & 0.9 & 5.6 & \pm & 0.8 & 5.3 & \pm & 1.6 & 7.2 & \pm & 1.3 & 5.7 & & 1.2 \\
\hline Tumor/Bone & 2.6 & \pm & 0.5 & 3.7 & \pm & 0.9 & 3.0 & \pm & 0.9 & 4.3 & \pm & 0.8 & 3.1 & \pm & 0.6 \\
\hline
\end{tabular}

Table S13-12. Tissue uptake (mean $\% \mathrm{ID} / \mathrm{g} \pm \mathrm{SD}$ ) of ${ }^{18} \mathrm{~F}-\mathrm{AlF}-\mathrm{NO} 2 \mathrm{~A}-\mathrm{cys}$ ar 3 administered via the lateral tail vein in female, C57B1/6 mice with orthotopic B16-F10 (melanoma) allografts and one group of female, BALB/c mice with orthotopic B16F10 (melanoma) allografts at $6 \mathrm{~h}$. *BALB/c time point. 


\section{${ }^{18}$ F-AIF-NO2A-cysVar3 in U87MG tumor-bearing male nude mice}

\begin{tabular}{lrl}
\hline Tissue & \multicolumn{2}{c}{$\mathbf{6 ~ h}(\mathbf{n}=\mathbf{6})$} \\
\hline Blood & 5.34 & \pm 2.40 \\
Heart & 0.73 & \pm 0.15 \\
Lungs & 0.91 & \pm 0.15 \\
Liver & 5.59 & \pm 0.72 \\
Spleen & 0.24 & \pm 0.06 \\
Pancreas & 0.21 & \pm 0.04 \\
Stomach & 0.46 & \pm 0.08 \\
S. Intestine & 2.13 & \pm 0.20 \\
L. Intestine & 1.15 & \pm 0.18 \\
Kidneys & 10.1 & \pm 1.1 \\
Muscle & 0.15 & \pm 0.03 \\
Bone & 0.079 & \pm 0.014 \\
Skin & 0.39 & \pm 0.07 \\
Brain & 0.151 & \pm 0.018 \\
U87MG Tumor & 0.49 & \pm 0.23 \\
\hline
\end{tabular}

Table S13-13. Tissue uptake (mean \%ID \pm SD) of ${ }^{18} \mathrm{~F}-\mathrm{AlF}-\mathrm{NO} 2 \mathrm{~A}$-cysVar3 administered via the lateral tail vein in male, nude mice with U87MG xenografted flank tumors at $6 \mathrm{~h}$. ${ }^{*} \mathrm{BALB} / \mathrm{c}$ time point. 


\begin{tabular}{lccc}
\hline Tissue & \multicolumn{3}{c}{ 6 h } \\
(n=6) \\
\hline Blood & 10.7 & \pm & 0.9 \\
Heart & 4.17 & \pm & 0.42 \\
Lungs & 4.57 & \pm & 0.81 \\
Liver & 4.34 & \pm & 0.88 \\
Spleen & 2.30 & \pm & 0.34 \\
\hline Pancreas & 1.30 & \pm & 0.24 \\
Stomach & 0.70 & \pm & 0.24 \\
S. Intestine & 1.20 & \pm & 0.21 \\
L. Intestine & 0.50 & \pm & 0.09 \\
\hline Kidneys & 17.4 & \pm & 2.5 \\
Muscle & 0.88 & \pm & 0.08 \\
Bone & 1.27 & \pm & 0.11 \\
Skin & 2.43 & \pm & 0.30 \\
Brain & 0.38 & \pm & 0.04 \\
U87MG Tumor & 3.97 & \pm & 0.56 \\
\hline Tumor-to-tissue ratios (rel. u.) \\
Tumor/Blood & 0.37 & \pm & 0.06 \\
\hline Tumor/Liver & 0.9 & \pm & 0.2 \\
Tumor/S. I. & 3.3 & \pm & 0.7 \\
\hline Tumor/L. I. & 7.9 & \pm & 1.8 \\
Tumor/Kidney & 0.23 & \pm & 0.05 \\
\hline Tumor/Muscle & 4.5 & \pm & 0.8 \\
Tumor/Bone & 3.1 & \pm & 0.5 \\
\hline Tumor/Brain & 10.5 & \pm & 1.9 \\
\hline
\end{tabular}

Table S13-14. Tissue uptake (mean $\% \mathrm{ID} / \mathrm{g} \pm \mathrm{SD}$ ) of ${ }^{18} \mathrm{~F}-\mathrm{AlF}-\mathrm{NO} 2 \mathrm{~A}-\mathrm{cys} V$ ar3 administered via the lateral tail vein in male, nude mice with U87MG xenografted flank tumors at $6 \mathrm{~h} . * \mathrm{BALB} / \mathrm{c}$ time point. 


\section{SUPPORTING INFORMATION REFERENCES}

1. Zeglis, B. M.; Sevak, K. K.; Reiner, T.; Mohindra, P.; Carlin, S. D.; Zanzonico, P.; Weissleder, R.; Lewis, J. S. (2013) A Pretargeted PET Imaging Strategy Based on Bioorthogonal Diels-Alder Click Chemistry. J. Nuc. Med. 54 (8), 1389-1396.

2. Dijkgraaf, I.; Franssen, G. M.; McBride, W. J.; D’Souza, C. A.; Laverman, P.; Smith, C. J.; Goldenberg, D. M.; Oyen, W. J. G.; Boerman, O. C. (2012) PET of Tumors Expressing Gastrin-Releasing Peptide Receptor with an ${ }^{18}$ F-Labeled Bombesin Analog. J. Nuc. Med. 53 (6), 947-952.

3. (a) Viola-Villegas, N. T., Carlin, S. D., Ackerstaff, E., Sevak, K. K., Divilov, V., Serganova, I., Kruchevsky, N., Anderson, M., Blasberg, R. G., and Andreev, O. A. (2014) Understanding the pharmacological properties of a metabolic PET tracer in prostate cancer Proc. Natl. Acad. Sci. U. S. A. 111, 7254-7259, DOI: 10.1073/pnas.1405240111; (b) Vavere, A. L., Biddlecombe, G. B., Spees, W. M., Garbow, J. R., Wijesinghe, D., Andreev, O. A., Engelman, D. M., Reshetnyak, Y. K., and Lewis, J. S. (2009) A novel technology for the imaging of acidic prostate tumors by positron emission tomography Cancer Res. 69, 4510-4516, DOI: 10.1158/0008-5472.CAN-08-3781; (c) Daumar, P., Wanger-Baumann, C. A., Pillarsetty, N., Fabrizio, L., Carlin, S. D., Andreev, O. A., Reshetnyak, Y. K., and Lewis, J. S. (2012) Efficient ${ }^{18}$ F-labeling of large 37-amino-acid pHLIP peptide analogues and their biological evaluation Bioconjugate Chem. 23, 15571566, DOI: $10.1021 / \mathrm{bc} 3000222$ 


\title{
CHAPTER 3
}

Submitted for publication

\section{New pHLIPs for the Targeted Intracellular Delivery of Cargo Molecules to Tumors}

\author{
Linden C. Wyatt ${ }^{\mathrm{a}}$, Anna Moshnikova ${ }^{\mathrm{a}}$, Troy Crawford ${ }^{\mathrm{a}}$, \\ Donald M. Engelman ${ }^{\mathrm{b}, 1}$, Oleg A. Andreev ${ }^{\mathrm{a}, 1}$, Yana K. Reshetnyak ${ }^{\mathrm{a}, 1}$ \\ ${ }^{a}$ Physics Department, University of Rhode Island, Kingston, RI 02881; \\ ${ }^{\mathrm{b}}$ Department of Molecular Biophysics and Biochemistry, Yale, New Haven, CT \\ 06511 \\ ${ }^{1}$ To whom correspondence may be addressed. Emails: reshetnyak@uri.edu or \\ andreev@uri.edu or donald.engelman@yale.edu
}

Keywords: membrane-associated folding, tumor acidity, cytoplasmic drug delivery, polar drugs, targeted chemotherapy

Author Contributions: O.A.A., D.M.E., and Y.K.R. designed research; L.C.W., A.M., and T.C. performed research; L.C.W., A.M., and Y.K.R. analyzed data; and L.C.W., D.M.E., O.A.A., and Y.K.R. wrote the paper. 
Conflict of Interest Statement: D.M.E., O.A.A., and Y.K.R. are founders of pHLIP, Inc. They have shares in the company, but the company did not fund any part of the work reported in the paper, which was done in their academic laboratories.

\section{Significance Statement}

Two aspects of contemporary cancer chemotherapy are targeted therapy and targeted delivery. While there are many examples of targeted therapy, targeted delivery has been limited due to its reliance on tumor cell biomarkers, either in the form of antigens or of specific binding sites; because of the dependence on the presence of these suitable biomarkers and because of high tumor heterogeneity, targeted delivery has not shown great success so far. The emergence of pHLIP technology provides an alternative by targeting a metabolic marker: tumor cell surface acidity. While promising, the technology is still developing and advances could have a significant impact on therapy. Here, we report several new pHLIPs, including pHLIP bundles, a new concept, and we thoroughly evaluate these constructs alongside a new generation of pHLIPs. We also discuss challenges inherent to the design and accurate evaluation of pHLIPs. Our research elucidates the strengths and weaknesses of existing pHLIPs, proposes future peptide modifications that could further improve tumor targeting, and discusses the applicability of this new generation of pHLIPs for specific areas of drug delivery. These principles and new constructs promise increasing success in tumor therapy. 


\begin{abstract}
pH (Low) Insertion Peptides (pHLIPs) target acidity at the surfaces of cancer cells and show utility in a wide range of applications including tumor imaging and the intracellular delivery of therapeutic agents. Here we report new pHLIP constructs that significantly improve the targeted delivery of agents into tumor cells. The new constructs include pHLIP bundles (conjugates consisting of two or four pHLIP peptides linked by polyethelyne glycol) and Var3 pHLIPs containing either the nonstandard amino acid $\gamma$-carboxyglutamic acid or a glycine-leucine-leucine motif. The performance of the new constructs in vitro and in vivo was compared with previous pHLIP variants. A wide range of experiments was performed on nine constructs including: i) biophysical measurements using steady-state and kinetic fluorescence spectroscopy, circular dichroism (CD) spectroscopy, and oriented circular dichroism (OCD) spectroscopy to study the $\mathrm{pH}$-dependent insertion of pHLIP variants across the membrane lipid bilayer; ii) cell viability assays to gauge the $\mathrm{pH}$-dependent potency of peptide-toxin constructs by assessing the intracellular delivery of the polar, cellimpermeable cargo molecule amanitin at physiological and low $\mathrm{pH}(\mathrm{pH} 7.4$ and 6.0, respectively); and iii) tumor targeting and biodistribution measurements using fluorophore-peptide conjugates in a breast cancer mouse model. The main principles of the design of pHLIP variants for a range of medical applications are discussed.
\end{abstract}




\section{Introduction}

The targeted delivery of drugs to cancer cells promises to maximize their therapeutic effects while reducing side effects. Although many biomarkers exist that can be exploited to improve tumor targeting and treatment outcomes, such as various receptors overexpressed at the surfaces of some cancer cells, useful markers are not

present in all tumors. Further, the heterogeneity of the cancer cell population in an individual tumor and between tumors of various patients limits the effective use of biomarker targeting technologies, and rapid mutation increases the likelihood of the selection of cancer cell phenotypes that do not express high levels of the targeted biomarker. Thus, biomarker targeting can act as a selection method that may lead to the development of drug resistance and poor patient outcomes (1-3).

It is well known that acidosis is ubiquitous in tumors, including both primary tumors and metastases, as a consequence of their rapid metabolism (4). The acidic microenvironment is generated by the increased use of glycolysis by cancer cells, and by the abundance of carbonic anhydrase proteins on the cancer cell surfaces. Tumor cells stabilize their cytoplasmic $\mathrm{pH}$ by exporting the acidity to the extracellular environment. As a result of the proton flux and resulting membrane potential, the extracellular $\mathrm{pH}$ is lowest at the surfaces of cancer cells, where it is significantly lower than both the normal physiological $\mathrm{pH}$ and the bulk extracellular $\mathrm{pH}$ in tumors (5-7). The low $\mathrm{pH}$ region persists at the cancer cell surface even in well-perfused tumor areas. The acidity on the surfaces of cancer cells is a targetable characteristic that is not subject to clonal selection, and the level of acidity is a predictor of tumor invasion and aggression since more rapidly growing tumor cells are more acidic. 
The emerging technology based on $\mathrm{pH}$ (low) insertion peptides (pHLIPs) comprises a variety of acidity-targeting peptides, each possessing different tumortargeting characteristics. pHLIPs can be used in a wide variety of applications, so it is desirable to have a range of options available for use in specific applications. Some examples of these applications include: i) fluorescence imaging (8-10) and fluorescence image-guided surgery (11); ii) nuclear imaging, including positron emission tomography (PET) and single-photon emission computed tomography (SPECT) $(12,13)$; iii) therapy, including the targeted delivery of polar toxins that cannot cross cell membranes $(14,15)$, drug-like molecules that inherently diffuse across cell membranes $(16,17)$, and gene therapy (18); and iv) nanotechnology, in which pHLIP is used to enhance the delivery of gold nanoparticles $(19,20)$ or liposome-encapsulated payloads to cancer cells (21).

pHLIPs are triggered to insert across the membranes of cancer cells by the acidity at the cancer cell surface. The behavior of peptides in the pHLIP family is typically described in terms of three states: at physiological $\mathrm{pH}(\mathrm{pH} 7.4)$, peptides exist in equilibrium between a solvated state (State I) and a membrane-adsorbed state (State II); a decrease in $\mathrm{pH}$ shifts the equilibrium toward a membrane-inserted state (State III) (22). The mechanism of action of peptides in the pHLIP family is well understood: protonatable residues, which are interspersed throughout the hydrophobic middle region and the $\mathrm{C}$-terminal, membrane-inserting region of the peptides, are negatively charged at physiological $\mathrm{pH}$ but become neutralized by protonation with a decrease in $\mathrm{pH}$. The loss of charge and increase in overall hydrophobicity drives pHLIPs to partition across the hydrophobic core of the membrane bilayer to form a 
transmembrane helix. This helix spans the lipid bilayer, leaving the $\mathrm{N}$-terminus in the extracellular space and placing the C-terminus in the intracellular space where, due to the more alkaline $\mathrm{pH}$ in the cytosol, the $\mathrm{C}$-terminus can again become deprotonated and charged, stably anchoring the peptide in the cell membrane.

Following an extensive characterization of wild-type (WT) pHLIP, a firstgeneration of pHLIP variants was created to examine the effects on targeting due to fairly straightforward changes to the WT primary structure such as sequence truncation, the addition and replacement of some protonatable residues with others, and sequence reversal (23-25). Importantly, a number of the changes that were investigated had adverse effects on pHLIP properties, suggesting that further studies might reveal design principles and give more useful molecules for targeted therapy. Of these first-generation variants, Variant 3 (Var3) appeared to have the most desirable insertion characteristics and much research has been focused on the use of Var3 for various applications $(10,11,26,27)$.

Lately, new variants have emerged that incorporate more innovative changes to the peptide primary structure; these changes include the use of the non-standard amino acids $\gamma$-carboxyglutamic acid (Gla), a residue with two protonatable carboxyl groups, and $\alpha$-aminoadipic acid (Aad), a more hydrophobic version of the glutamic acid residue (28), as well as the creation of a pHLIP peptide de novo, ATRAM (29). Here, we examine several new members of the pHLIP family of peptides, including pHLIP bundles, compare their biophysical properties to some of the previous generation variants, and evaluate the utility of nine pHLIPs in drug delivery and tumor imaging 
applications. These variants significantly expand the useful range of applications in targeted cancer therapy.

\section{Results}

pHLIP constructs. We have designed two new pHLIP variants, Var3/Gla (incorporating the non-standard amino acid Gla) and Var3/GLL (utilizing a glycineleucine-leucine motif), and a novel construct: pHLIP bundles. pHLIP bundles consist of two- or four-armed $2 \mathrm{kDa}$ polyethylene glycol (PEG) spacers conjugated to the cysteine residue at the N-terminus of WT: PEG-2WT (Figure 1a) and PEG-4WT (Figure $1 b$ ), respectively. Our motivation is to increase both the membrane affinity and the cooperativity of the transition from the membrane-surface state to the membrane-inserted state. Enhancement of affinity is expected to improve targeting, and higher cooperativity should narrow the $\mathrm{pH}$ window that produces transmembrane drug delivery. The nine pHLIP variants studied (molecular weights and retention times are provided in Table S2) were as follows, with additional variations from the addition of single $\mathrm{N}$ - or $\mathrm{C}$-terminal cysteine or lysine residues for conjugation purposes (a list of all peptides investigated is provided in Table S1):

WT:

AEQNPIYWARYADWLFTTPLLLLDLALLVDADEGT

PEG-2WT: $\quad$ Two-arm PEG conjugated to two WT pHLIPs

PEG-4WT: $\quad$ Four-arm PEG conjugated to four WT pHLIPs

WT/Gla:

AEQNPIYWARYAGlaWLFTTPLLLLDLALLVDADEGT 
WT/Gla/Aad: $\quad$ AEQNPIYWARYAGlaWLFTTPLLLLAadLALLVDADEGT

Var3:

ADDQNPWRAYLDLLFPTDTLLLDLLW

Var3/Gla:

ADDQNPWRAYLGlaLLFPTDTLLLDLLW

Var3/GLL:

GEEQNPWLGAYLDLLFPLELLGLLELGLWG

ATRAM:

\section{GLAGLAGLLGLEGLLGLPLGLLEGLWLGLELEGN}

These pHLIP variants can be grouped together in various ways by shared characteristics. A WT-like group contains peptides with two protonatable residues (shown in bold) in the underlined putative transmembrane (TM) region, multiple protonatable residues in the membrane-inserting $\mathrm{C}$-terminal region, and two tryptophan residues (residue $\mathrm{W}$ ) located at the beginning of the helix-forming TM region; this group includes WT, PEG-2WT and PEG-4WT, WT/Gla, and WT/Gla/Aad. A Var3-like group is based on Var3 from the first generation of pHLIP variants (25). This group includes Var3, Var3/Gla and Var3/GLL, each of which have three protonatable residues in the TM region and tryptophan residues located at the beginning and end of the TM region. Considering this scheme, ATRAM, with its multiple glycine and leucine residues and single tryptophan located about two-thirds of the way through its TM region, is in a group of its own. Other subgroups can be considered as well: a subgroup of peptides that incorporate the non-standard Gla residue, shown in italics (i.e., WT/Gla, WT/Gla/Aad, and Var3/Gla), and another subgroup that includes peptides containing the GLL motif (Var3/GLL and ATRAM).

When performing analysis of biophysical measurements, analyzing variants with respect to their group-mates becomes important: the very different characteristics 
of peptides from various groups make it difficult to accurately compare the behavior of all peptides at the same time.

Biophysical steady-state and kinetics studies. A variety of spectroscopic techniques were employed to probe the interaction between pHLIP variants and POPC phospholipid bilayers in liposomes; these techniques included steady-state fluorescence spectroscopy, circular dichroism (CD) spectroscopy, oriented circular dichroism (OCD) spectroscopy, and stopped-flow fluorescence measurements. Steadystate fluorescence and $\mathrm{CD}$ experiments were conducted in phosphate buffer titrated with hydrochloric acid to drop the $\mathrm{pH}$ from $\mathrm{pH} 8$ to $\mathrm{pH} 4$ to ensure consistency with previously published data $(25,29,30)$. Steady-state and kinetics fluorescence experiments measuring the $\mathrm{pH}-$ dependent transition from State II to State III were carried out in phosphate buffer containing physiological concentrations of calcium $(1.25 \mathrm{mM})$ and magnesium $(0.65 \mathrm{mM})$ ions (the concentrations at which free calcium and magnesium ions are found in the blood), since we conjectured that some of the studied constructs might interact with these ions, and that this interaction might significantly affect the ultimate in vivo performance of the peptides.

We established that, in solution, PEG-2WT and PEG-4WT most probably exist in compact coil conformations, where tryptophan and other aromatic residues can form stacking structures. The resulting exciton formation was seen as a minimum around $230 \mathrm{~nm}$ in the CD spectra of these pHLIP bundles (Figure $1 e$ and $1 f$ ). At $\mathrm{pH} 8$, changes in tryptophan fluorescence show that both constructs interact with the lipid bilayer and it appears that PEG-4WT exhibits stronger binding than PEG-2WT in 
State II. With a reduction of $\mathrm{pH}$, both $\mathrm{pHLIP}$ bundles inserted into the bilayer to form helices, and the transmembrane orientations of these helices were confirmed by OCD measurements (Figure $1 g$ and $1 \mathrm{~h}$ ). It is important to note that in State III, the membrane-inserted state, the exciton signal generated by $\pi-\pi$ stacking is no longer present, suggesting that the insertion of each pHLIP renders it independent of the other(s). The apparent $\mathrm{p} K$ of the transition from State II to State III was shifted to $\mathrm{pH}$ 6.6 and, as might be expected, the cooperativity of the transition was increased for PEG-4WT compared to PEG-2WT (Figure $1 i$ and $1 j$ ).

We compared the groups consisting of WT, Var3, and ATRAM pHLIP variants to the newly designed Var3/Gla and Var3/GLL pHLIP variants. The retention times of the peptides indicate increasing hydrophobicity within the groups in the following order, from less to more hydrophobic: WT, WT/Gla, WT/Gla/Aad and Var3, Var/Gla, Var3/GLL, and ATRAM, with ATRAM being the most hydrophobic (Table S2). Both new pHLIP variants, Var3/Gla and Var3/GLL, demonstrated a pHdependent interaction with the membrane (Figure S1). Var3/GLL showed a higher percentage of membrane-inserted population at $\mathrm{pH} 8$, which reflects a higher affinity of the peptide for the lipid bilayer both at physiological and high $\mathrm{pH}$ due to the increased hydrophobicity of the peptide.

As seen for previous pHLIP designs, a blue shift (or decrease in Stokes shift) resulting from the environmental changes from State I to State II and State III was observed for all peptides (Table S3), indicating partitioning of the peptides into the lipid bilayer. However, we cannot directly compare the positions of fluorescence spectra maxima for peptides belonging to the different groups, since the locations of 
the tryptophan residues within the peptides varies greatly. With this fact in mind, we can conclude that the peptides had very different conformations in State II at $\mathrm{pH} 8$, and that the highest membrane affinity was exhibited by the PEG-pHLIPs and by the WT/Gla/Aad, Var3/GLL, and ATRAM peptides. The PEG-pHLIPs have multiple binding sites due to the linking of multiple WT peptides within a single construct, which is expected to enhance binding affinity. The WT/Gla/Aad, Var3/GLL, and ATRAM have the most hydrophobic sequences, and thus exhibit strong binding/insertion. We also found that some peptides were especially sensitive to the presence of calcium and magnesium ions, namely WT, variants containing the Gla residue (WT/Gla, WT/Gla/Aad, and Var3/Gla) and ATRAM. This sensitivity was most obviously seen as a decreased Stokes shift (usually 2-3 nm) in State I and/or State II, and might reflect slight increases in the hydrophobicity of the peptides caused by the coordination of divalent cations resulting from the presence of closely spaced protonatable residues, such as those found in the $\mathrm{C}$-terminal region of WT and, to some degree, in ATRAM, or to the presence of the Gla residue, with its two protonatable carboxyl groups, in the WT/Gla, WT/Gla/Aad, and Var3/Gla peptides. It is known that a Gla residue can form a complex with a calcium ion (31-33). The decrease in Stokes shift in State II is likely due to the location of membrane-adsorbed peptides deeper in the lipid membrane (especially for the more hydrophobic pHLIPs: WT/Gla/Aad, Var3/GLL, and ATRAM) and/or a shift in peptide population from the solvated to the membrane-adsorbed state.

In contrast to tryptophan fluorescence changes, which are dependent on the location of tryptophan residues within the peptide sequence, the appearance of helicity 
is a more general parameter which can be compared among all peptides. In Figure $2 a$ (and Table S3), we give the ratio of ellipticity at $205 \mathrm{~nm}$ to $222 \mathrm{~nm}$, an indicator of the degree of helicity (lower ratios indicate higher helicity), obtained for different peptides in different states. In State I, the lowest ratios were observed for pHLIP bundles, which correlate with the appearance of the exciton signal at $230 \mathrm{~nm}$. In State II, the least unstructured peptides (ratio <1.5) were PEG-4WT, WT/Gla/Aad, Var3/GLL, ATRAM, and PEG-2WT, which exhibited a higher affinity to the membrane and an increase in the peptide-inserted population at $\mathrm{pH}$ 8. At low $\mathrm{pH}$, all peptides exhibited similar helical content, as expected from the observed formation of TM helices.

The transitions from State II to State III seen in steady-state and kinetics modes exhibited $\mathrm{pK}$ values in the range of $\mathrm{pH} 5.7$ to 6.6 in the presence of physiological concentrations of calcium and magnesium ions, with the highest cooperativity observed for PEG-4WT, and transition times varying from 0.1 to $37.5 \mathrm{~s}$ (Table 1). There are subtleties that affect the comparison and interpretation of the data: i) the peptides are in different starting conditions in State II at $\mathrm{pH} 8$ due to greatly differing overall peptide hydrophobicity; ii) $\mathrm{p} K$ values and characteristic times report the movement of tryptophan residues into environments inside the membrane; however, since the tryptophan residues are located in different regions of each pHLIP, their propagation into the membrane as measured via changes in fluorescence parameters should be expected to be different; and iii) the cooperativity of the transition is a somewhat unstable parameter in the fitting of experimental $\mathrm{pH}$-dependence data using the Henderson-Hasselbalch equation, especially if slopes are introduced at the initiation and completion of the transition (34). Low values of cooperativity $(n<1)$ 
were observed for the peptides with tryptophan residues located at (Var3 group) or close (ATRAM) to the C-terminus, which must be translocated across the cell membrane. ATRAM and Var3/GLL, which are the most hydrophobic pHLIPs and are therefore likely to be located more deeply than others in the membrane at $\mathrm{pH} 8$, demonstrated the fastest times of insertion. As we showed previously, the removal of protonatable residues from the inserting $\mathrm{C}$-terminus increases the rate of the transition from State II to State III $(24,25)$. Thus, the group of Var3-like peptides exhibited fast insertion times $(\mathrm{t}<1 \mathrm{~s})$. In the group of WT peptides, the time of insertion decreased as the hydrophobicity of the peptide increased, with insertion times listed in the following order (from longest to shortest time of insertion): WT, WT/Gla, WT/Gla/Aad, PEG-2WT, and PEG-4WT.

Intracellular delivery of polar cargo. Before studying the toxicity of pHLIP-toxin conjugates, we investigated whether the pHLIP bundles could cause any acute cytotoxicity by themselves. HeLa cells were treated with either PEG-2WT or PEG4WT at physiological $\mathrm{pH}(\mathrm{pH}$ 7.4) and low $\mathrm{pH}(\mathrm{pH}$ 6.0) for two hours. We did not observe any cytotoxic effect at either $\mathrm{pH}$, even when treating with concentrations up to $10 \mu \mathrm{M}$ (construct concentration is presented as concentration of WT pHLIP).

A proliferation assay was employed to evaluate the ability of pHLIPs to deliver the amanitin toxin, a relatively cell-impermeable, polar cargo molecule $(35,36)$. For amanitin to induce cytotoxicity it must be translocated across the cell membrane, be released from its peptide carrier, and reach its target in the nucleus (RNA polymerase II). Amanitin was conjugated via a cleavable disulfide link to the inserting, C-termini 
of the peptides. The translocation capabilities of the pHLIP-amanitin conjugates were measured as the inhibition of proliferation of HeLa cells due to treatment with increasing concentrations (up to $2 \mu \mathrm{M}$ ) of pHLIP-amanitin at either physiological $\mathrm{pH}$ ( $\mathrm{pH}$ 7.4) or low $\mathrm{pH}(\mathrm{pH}$ 6.0) for two hours, followed by removal of the constructs, transfer of cells to normal cell culture media, and assessment of cell death at 48 hours.

Each of the conjugates demonstrated pH-dependent cytotoxicity (Figure S2). The calculated $E C_{20}, E C_{50}$, and $E C_{80}$ at physiological and low $\mathrm{pH}$ are shown in Table 1. At low $\mathrm{pH}$, the most potent constructs were the pHLIP bundles, which exhibited the highest cooperativity of insertion into the membrane. The least toxic at normal $\mathrm{pH}$ among all constructs was Var3. Figure $2 b$ lists the therapeutic indexes (TI), defined as the ratio of $E C_{50}$ at $\mathrm{pH} 7.4$ to $E C_{50}$ at $\mathrm{pH} 6.0$ for each case. A TI of about 9 was obtained for WT/Gla and Var3, and the TI was around 5.5 for PEG-2WT, Var3/Gla and ATRAM. It is also desirable to have high potency, which is defined as the difference between cell viability at low and physiological $\mathrm{pHs}$ at different concentrations of the construct (Figure 3). All constructs had high potency (60-70\%) at particular concentrations; however, just a few constructs, namely Var3, Var3/Gla, and WT/Gla, had a high, stable potency over a wide range of concentrations. pHLIP bundles displayed the highest potency at the lowest concentrations $(0.1-0.2 \mu \mathrm{M})$. The potency of ATRAM peaked at concentrations around $0.5 \mu \mathrm{M}$ and declined sharply at higher concentrations; this decline is most likely associated with the increased hydrophobicity of ATRAM, which results in a high affinity to the cell membrane at normal and high $\mathrm{pH}$ and promotes the shift in equilibrium toward the membrane- 
inserted form that is associated with the translocation of cargo across the cell membrane.

Tumor targeting. To evaluate the tumor targeting and biodistribution characteristics of the pHLIP variants, we conjugated the fluorescent dye Alexa Fluor 546 (AF546) to the non-inserting, N-termini of seven of the peptides. Our previous data indicate excellent tumor targeting by AF546-pHLIPs $(9,27)$. In the case of pHLIP bundles, AF546 was conjugated to the inserting, C-termini of the PEG-2WT and PEG-4WT pHLIPs, as the N-termini were occupied by PEG polymers. A well-established mouse model, using implanted cells of acidic 4T1 murine breast tumors, was used in the study; this model is targeted well by pHLIPs $(9,27)$. Following the development of breast tumors in the mouse flank, each fluorescent construct was introduced by a single tail vein injection. Animals were euthanized four hours after the injection of the fluorescent conjugates, and the tumor and major organs (kidney, liver, lungs, spleen, and muscle) were collected and imaged. We selected the four-hour post-injection time point based on previous pharmacokinetics data which show that the highest tumor targeting of pHLIPs is observed four hours after the injection of construct $(9,27)$. The mean values of the surface fluorescence intensity of tumors, muscle, and organs are given in Table S4. The normalized tumor fluorescence intensity (normalized by tumor uptake of AF546-WT) for all constructs is shown in Figure $4 a$. The highest tumor targeting was observed for the Var3 construct, as well as for Var3/Gla and ATRAM. The tumor uptakes of the WT and Var3/GLL constructs were significantly reduced, by 1.6- and 2.6-fold, respectively, compared to the uptake of Var3. The uptakes of 
WT/Gla, WT/Gla/Aad, and the pHLIP bundles were reduced even further compared to the uptake of the WT construct. It is possible that the decreased tumor targeting observed in the PEG-pHLIP bundles might be attributed to the fact that the AF546 dye was conjugated to the $\mathrm{C}$-terminus, which is translocated into the cytosol. At the same time, the tumor-to-muscle ratio of the WT-like group was in the range of 5.4-7.5. The highest tumor-to-muscle ratios were observed for $\operatorname{Var} 3(\mathrm{~T} / \mathrm{M}=8.9)$ and PEG-2WT ( $\mathrm{T} / \mathrm{M}=7.5$ ), and the lowest ratio was observed in Var3/GLL (T/M=4.0) (Figure $4 b$ and Table S5). Among all constructs, only Var3/GLL demonstrated a tumor-to-kidney ratio less than 1 (Figure $4 c$ and Table S5). The highest tumor-to-liver ratio was found in Var3 and Var3/Gla (Figure 4d and Table S5).

Because PEG-2WT-AF546 and PEG-4WT-AF546 are several times larger than the other pHLIP variants, we hypothesized that they might have slower pharmacokinetics. Therefore, we also performed imaging at the 24-hour post-injection time point for the two PEG-pHLIP conjugates; however, we did not observe any significant signal increase in tumors at 24 hours post-injection compared to 4 hours post-injection (Table S4).

\section{Discussion}

In order to advance cancer therapy using a range of agents with different properties, we have developed new pHIP variants and pHLIP bundles, and compared their performance to the performance of recently introduced variants with non-standard amino acids (Gla and Aad) and the hydrophobic glycine-leucine-leucine (GLL) motif. Our goal was to correlate the biophysical properties of the membrane interactions of 
different pHLIPs, including physiological concentrations with free calcium and magnesium ions, to the ability of these pHLIPs to move polar cargo across the cell membrane and to target acidic tumors.

The insertion $\mathrm{p} K$ and cooperativity parameters of the steady-state, $\mathrm{pH}$ dependent transition from State II at $\mathrm{pH} 8$ to State III at $\mathrm{pH}<5$ can be taken as predictors of the performance of a pHLIP for drug delivery and tumor targeting (17, 28, 29). While $\mathrm{p} K$ is a rather stable fitting parameter, the cooperativity parameter (Hill coefficient) might vary over a wide range resulting from different fittings which are within the level of accuracy of the experimental measurements. Moreover, if different binding affinities are assumed, the Hill model loses validity. In general, highly cooperative transitions are hard to measure in biological systems with noise, especially when examining relatively short peptides like those that comprise the class of pHLIP peptides (28). Only if the biological system is approximated to be infinite can a phase transition occur (37). Moreover, transition parameters for different peptides can only be truly compared when both peptides have precisely the same starting and ending states; although this condition is met for the membrane-inserted state (State III) of the peptides, which appears very similar among pHLIP variants, the condition that the initial state (State II) of the peptides be identical is not met. As hydrophobicity varies widely among peptides of the pHLIP family due to the difference in numbers of protonatable, polar, and hydrophobic residues and their location within the peptide sequences, the characteristics of the peptide population in the initial state of the transition also varies as these peptides position themselves at different interaction levels with the hydrophobic/hydrophilic boundary region of a bilayer. 
The population percentages of inserted peptide presented in Table S6 were calculated from the $\mathrm{pH}$-dependent transitions of pHLIP variants. The numbers represent the percentage of membrane-inserted peptides at varying $\mathrm{pH}$ assuming that, at the beginning of the transition (State II) (i.e., at physiological $\mathrm{pH}$ and higher), the population of inserted peptides is close to zero. In reality, close consideration of the interaction between a pHLIP variant and the membrane at $\mathrm{pH} 8$, in conditions more alkaline than physiological conditions where the inserted peptide population should be even less than at physiological conditions, indicates that the most hydrophobic sequences, such as ATRAM and Var3/GLL, and bundled pHLIPs with multiple binding sites within a single construct, exhibit a significant inserted peptide population, as shown by the loss of $\mathrm{pH}$-dependent differences in the translocation of the polar, cell-impermeable cargo amanitin with an increase in construct concentration (i.e., a decrease in potency at higher concentrations). Due to patient variability, it is highly desirable that potential therapeutic pHLIP constructs be able to discriminate between healthy and tumor tissue over a wide concentration range, meaning that a constant potency is necessary to avoid targeting normal tissue and the resulting significant side effects, which suggests that these variants (i.e., ATRAM, Var3/GLL, and bundled pHLIPs) may not be well suited for clinical development using agents that require tight targeting.

In addition to the steady-state experiments, it is important to probe tumor targeting and to examine the biodistribution of the constructs when injected into the high-flowrate blood stream, since targeted delivery is always diminished by clearance via the blood. The best tumor targeting was shown by faster-inserting pHLIP 
constructs; thus, in the design of new pHLIP variants, the biophysical kinetics parameters need to be considered in addition to the more traditionally prioritized steady-state properties. These kinetics parameters might be especially critical for the delivery and translocation of a cargo across a membrane, since we have shown that charges and the presence of cargo at the inserting end of a pHLIP can slow the process of insertion (24). Different cargoes linked to a pHLIP alter biodistribution and tumor targeting (27). Less polar pHLIP variants conjugated with hydrophobic cargoes might have a higher tendency toward targeting normal tissue and hepatic clearance. On the other hand, the size of links in pHLIP bundles could be used to tune biodistribution and redirect clearance from renal to hepatic.

Among the pHLIP variants we investigated, Var3 demonstrated excellent performance in vitro (displaying the most stable potency over a wide range of concentrations) and in vivo, displaying high tumor targeting. Variants containing the Gla residue, especially the WT/Gla construct, showed an increase in the cooperativity of the membrane insertion transition as previously reported (28), and showed an improved therapeutic index; however, the tumor targeting of WT/Gla was lower compared to the tumor targeting of WT.

$\gamma$-carboxyglutamic acid is not naturally encoded in the human genome, but is introduced into proteins through the post-translational carboxylation of glutamic acid, resulting in an amino acid with two carboxyl groups. Many proteins are known to have Gla-rich domains, including several coagulation factors which, by coordinating calcium ions, are induced to change conformation and increase their hydrophobicity and affinity to the cell membrane bilayer (38). Calcium complex formation by a 
pHLIP increases the hydrophobicity of the peptide and alters the interaction between peptide and membrane; this fact, along with the fact that the cost of synthesizing a Gla-containing peptide is very high (Gla is one of the most expensive amino acids) might somewhat reduce enthusiasm for using the Gla residue, although if there were sufficient advantages in a specific case the cost might be justified. While considering peptide synthesis, it is worthwhile to note that very hydrophobic pHLIP sequences (like ATRAM), especially when coupled with even moderately hydrophobic cargoes, might be challenging to produce in the large quantities needed for clinical translation.

There is no single recipe for the best pHLIP: the peptide will need to be tailored to each specific medical application. For example, kidney clearance might be preferred to liver clearance for PET-pHLIP imaging constructs (13). High tumor-tonormal tissue fluorescence intensity ratios will be the key in fluorescence-guided surgery applications (11). Delivery of highly toxic molecules, such as amanitin, would require minimal off-targeting, thus high potency and therapeutic index will be critical. However, for the delivery of polar peptide nucleic acids (PNAs) or other highly specific inhibitors of particular pathways in cancer cells, neither of which are associated with toxicity in normal cells, the requirement to reduce off-targeting might be much lower and the emphasis would be shifted toward the efficiency of delivery, the goal being to translocate as much cargo as possible $(18,39)$. pHLIP bundles might yield excellent results in these types of applications, supported by the observation that PEG-4WT was the most efficient at delivering the polar molecule amanitin to the intracellular space. We suspect that bundling multiple Var3 pHLIPs, in the same fashion that we linked two or four WT pHLIPs, might be even more advantageous. 
Var3 demonstrates membrane insertion rates orders of magnitude faster than the insertion rates of WT; with the knowledge that faster insertion rates observed in biophysical experiments correlates to better tumor targeting in vivo, it stands to reason that potential PEG-Var3 constructs might demonstrate better tumor targeting still.

In drug delivery applications, pHLIP peptides are best designed for the delivery of polar, cell-impermeable molecules $(14,35,40,41)$. The intracellular delivery of a polar cargo could be further tuned by altering the link connecting the cargo to pHLIP and/or by attaching modulator molecules to the inserting end of the peptide $(14,15,18,35)$. Additionally, pHLIP could be used for the targeted delivery of cell-permeable, drug-like molecules since it can significantly increase the time of retention in blood, positively alter the biodistribution of drugs that typically rely on passive diffusion, and enhance tumor targeting, all of which would lead to an increase in therapeutic index (16). More polar pHLIP variants are expected to be better suited to applications involving the intracellular delivery of cell-permeable cargoes.

We have now established a set of properties for a number of pHLIPs, which can be selected as starting points for clinical development in different circumstances. This body of work, along with the prior studies, opens pathways for targeted delivery using a range of imaging and therapeutic agents in the fight against cancer.

\section{Materials and Methods}

The details of experimental protocols can be found in Supplementary Materials and Methods. 


\section{Acknowledgements}

We are grateful to Dr. Dhammika Weerakkody for his assistance and helpful discussions. The research reported in this publication was supported in part by the National Institute of General Medical Sciences of the National Institutes of Health under award number R01GM073857 to OAA, YKR, and DME, and in part by the

Institutional Development Award (IDeA) Network for Biomedical Research Excellence from the National Institute of General Medical Sciences of the National Institutes of Health under grant number P20GM103430. 


\section{References}

1. Marusyk A \& Polyak K (2010) Tumor heterogeneity: causes and consequences. Biochim Biophys Acta 1805(1):105.

2. Gillies RJ, Verduzco D, \& Gatenby RA (2012) Evolutionary dynamics of carcinogenesis and why targeted therapy does not work. Nat Rev Cancer 12(7):487-493.

3. Lloyd MC, et al. (2016) Darwinian Dynamics of Intratumoral Heterogeneity: Not Solely Random Mutations but Also Variable Environmental Selection Forces. Cancer Res 76(11):3136-3144.

4. Estrella V, et al. (2013) Acidity generated by the tumor microenvironment drives local invasion. Cancer Res 73(5):1524-1535.

5. Zhang X, Lin Y, \& Gillies RJ (2010) Tumor pH and its measurement. J Nucl Med 51(8):1167-1170.

6. Hashim AI, Zhang X, Wojtkowiak JW, Martinez GV, \& Gillies RJ (2011) Imaging $\mathrm{pH}$ and metastasis. NMR Biomed 24(6):582-591.

7. Anderson M, Moshnikova A, Engelman DM, Reshetnyak YK, \& Andreev OA (2016) Probe for the measurement of cell surface $\mathrm{pH}$ in vivo and ex vivo. Proc Natl Acad Sci USA 113(29):8177-8181.

8. Reshetnyak YK, et al. (2011) Measuring tumor aggressiveness and targeting metastatic lesions with fluorescent pHLIP. Mol Imaging Biol 13(6):1146-1156.

9. Adochite RC, et al. (2014) Targeting breast tumors with $\mathrm{pH}$ (low) insertion peptides. Mol Pharm 11(8):2896-2905. 
10. Tapmeier TT, et al. (2015) The $\mathrm{pH}$ low insertion peptide pHLIP Variant 3 as a novel marker of acidic malignant lesions. Proc Natl Acad Sci USA 112(31):9710-9715.

11. Golijanin J, et al. (2016) Targeted imaging of urothelium carcinoma in human bladders by an ICG pHLIP peptide ex vivo. Proc Natl Acad Sci USA 113(42):11829-11834.

12. Macholl S, et al. (2012) In vivo pH imaging with (99m)Tc-pHLIP. Mol Imaging Biol 14(6):725-734.

13. Demoin DW, et al. (2016) PET imaging of extracellular $\mathrm{pH}$ in tumors with 64Cu- and 18F-labeled pHLIP peptides: A structure-activity optimization study. Bioconjugate Chem 27(9):2014-2023.

14. Andreev OA, et al. (2010) $\mathrm{pH}$ (low) insertion peptide (pHLIP) inserts across a lipid bilayer as a helix and exits by a different path. Proceedings of the National Academy of Sciences 107(9):4081-4086.

15. Wijesinghe D, Engelman DM, Andreev OA, \& Reshetnyak YK (2011) Tuning a polar molecule for selective cytoplasmic delivery by a pH (Low) Insertion Peptide. Biochemistry 50(47):10215-10222.

16. Burns KE, Robinson MK, \& Théveninr D (2015) Inhibition of cancer cell proliferation and breast tumor targeting of pHLIP-monomethyl auristatin E conjugates. Mol Pharm 12(4):1250-1258.

17. Burns KE, Hensley H, Robinson MK, \& Thevenin D (2017) Therapeutic efficacy of a family of pHLIP-MMAF conjugates in cancer cells and mouse models. Mol Pharm 14(2):415-422. 
18. Cheng CJ, et al. (2015) MicroRNA silencing for cancer therapy targeted to the tumor microenvironment. Nature 518(7537):107-110.

19. Yao L, et al. (2013) pHLIP peptide targets nanogold particles to tumors. Proc Natl Acad Sci USA 110(2):465-470.

20. Daniels JL, Crawford TM, Andreev OA, \& Reshetnyak YK (2017) Synthesis and characterization of pHLIP® coated gold nanoparticles. Biochem Biophys Rep 10:62-69.

21. Yao L, Daniels J, Wijesinghe D, Andreev OA, \& Reshetnyak YK (2013) pHLIP(®)-Mediated Delivery of PEGylated Liposomes to Cancer Cells. $J$ Control Release 167(3):228-237.

22. Reshetnyak YK, Segala M, Andreev OA, \& Engelman DM (2007) A Monomeric Membrane Peptide that Lives in Three Worlds: In Solution, Attached to, and Inserted across Lipid Bilayers. Biophys J 93(7):2363-2372.

23. Reshetnyak YK, Andreev OA, Segala M, Markin VS, \& Engelman DM (2008) Energetics of peptide (pHLIP) binding to and folding across a lipid bilayer membrane. Proc Natl Acad Sci USA 105(40):15340-15345.

24. Karabadzhak AG, et al. (2012) Modulation of the pHLIP transmembrane helix insertion pathway. Biophys J 102(8):1846-1855.

25. Weerakkody D, et al. (2013) Family of $\mathrm{pH}$ (low) insertion peptides for tumor targeting. Proc Natl Acad Sci USA 110(15):5834-5839.

26. Cruz-Monserrate Z, et al. (2014) Targeting Pancreatic Ductal Adenocarcinoma Acidic Microenvironment. Sci Rep 4:4410. 
27. Adochite RC, et al. (2016) Comparative study of tumor targeting and biodistribution of $\mathrm{pH}$ (low) insertion peptides (pHLIP peptides) conjugated with different fluorescent dyes. Mol Imaging Biol 113(42):11829-11834.

28. Onyango JO, et al. (2015) Noncanonical amino acids to improve the $\mathrm{pH}$ response of pHLIP insertion at tumor acidity. Angew Chem Int Edit 54(12):3658-3663.

29. Nguyen VP, Alves DS, Scott HL, Davis FL, \& Barrera FN (2015) A novel soluble peptide with $\mathrm{pH}$-responsive membrane insertion. Biochemistry 54(43):6567-6575.

30. Hunt JF, Rath P, Rothschild KJ, \& Engelman DM (1997) Spontaneous, pHdependent membrane insertion of a transbilayer alpha-helix. Biochemistry 36(49):15177-15192.

31. Cabaniss SE, Pugh KC, Pedersen LG, \& Hiskey RG (1991) Proton, calcium, and magnesium binding by peptides containing gamma-carboxyglutamic acid. Int J Pept Prot Res 37(1):33-38.

32. Shikamoto Y, Morita T, Fujimoto Z, \& Mizuno H (2003) Crystal structure of $\mathrm{Mg} 2+-$ and $\mathrm{Ca} 2+-$ bound Gla domain of factor IX complexed with binding protein. J Biol Chem 278(26):24090-24094.

33. Huang M, Furie BC, \& Furie B (2004) Crystal structure of the calciumstabilized human factor IX Gla domain bound to a conformation-specific antifactor IX antibody. J Biol Chem 279(14):14338-14346.

34. Barrera FN, et al. (2011) Roles of carboxyl groups in the transmembrane insertion of peptides. J Mol Biol 413(2):359-371. 
35. Moshnikova A, Moshnikova V, Andreev OA, \& Reshetnyak YK (2013) Antiproliferative effect of pHLIP-amanitin. Biochemistry 52(7):1171-1178.

36. Weerakkody D, et al. (2016) Novel pH-sensitive cyclic peptides. Sci Rep $6: 31322$

37. Sharma GP, Reshetnyak YK, Andreev OA, Karbach M, \& Müller G (2015) Coil-helix transition of polypeptide at water-lipid interface. J Stat MechTheory E P01034.

38. Kalafatis M, Egan JO, van't Veer C, \& Mann KG (1996) Regulation and regulatory role of gamma-carboxyglutamic acid containing clotting factors. Crit Rev Eukaryot Gene Expr 6(1):87-101.

39. Reshetnyak YK, Andreev OA, Lehnert U, \& Engelman DM (2006) Translocation of molecules into cells by $\mathrm{pH}$-dependent insertion of a transmembrane helix. Proc Natl Acad Sci USA 103(17):6460-6465.

40. Burns KE \& Thévenin D (2015) Down-regulation of PAR1 activity with a pHLIP-based allosteric antagonist induces cancer cell death. Biochem J 472(3):287-295.

41. Burns KE, McCleerey TP, \& Thévenin D (2016) pH-selective cytotoxicity of pHLIP-antimicrobial peptide conjugates. Sci Rep 6:28465.

42. Shen C, et al. (2008) The protein fluorescence and structural toolkit: Database and programs for the analysis of protein fluorescence and structural data. Proteins 71(4):1744-1754.

43. Schneider CA, Rasband WS, \& Eliceiri KW (2012) NIH Image to ImageJ: 25 years of image analysis. Nat Methods 9(7):671-675. 


\section{TABLES}

Table 1. The midpoint $(\mathrm{p} K)$, cooperativity $(n)$, and time $(t)$ parameters characterizing the $\mathrm{pH}$-dependent transition of $\mathrm{pHLIP}$ variants in the presence of POPC liposomes are presented. $E C_{20}, E C_{50}$, and $E C_{80}$ values were calculated for each pHLIP-amanitin construct at physiological and low $\mathrm{pH}$ by analyzing the $\mathrm{pH}$ - and concentrationdependent cell viability data (Figure S2).

\begin{tabular}{|c|c|c|c|c|c|c|c|c|c|}
\hline \multirow{2}{*}{ Peptide } & \multirow{2}{*}{$\mathrm{p} K$} & \multirow{2}{*}{$n$} & \multirow{2}{*}{$t(\mathbf{s})$} & \multicolumn{2}{|c|}{$E C_{20}(\mu \mathrm{M})$} & \multicolumn{2}{|c|}{$E C_{50}(\mu \mathrm{M})$} & \multicolumn{2}{|c|}{$E C_{80}(\mu \mathrm{M})$} \\
\hline & & & & pH 7.4 & pH 6.0 & pH 7.4 & pH 6.0 & pH 7.4 & pH 6.0 \\
\hline WT & 6.5 & 1.8 & 36.8 & 1.95 & 1.22 & 1.37 & 0.56 & 0.96 & 0.26 \\
\hline WT/Gla & 6.2 & 1.5 & 37.5 & 6.20 & 0.93 & 2.73 & 0.30 & 1.20 & 0.10 \\
\hline WT/Gla/Aad & 6.6 & 1.4 & 34.8 & 3.01 & 0.66 & 1.39 & 0.37 & 0.64 & 0.21 \\
\hline PEG-2WT & 6.6 & 1.8 & 18.8 & 1.98 & 0.54 & 1.03 & 0.19 & 0.53 & 0.07 \\
\hline PEG-4WT & 6.6 & 2.2 & 13.1 & 0.473 & 0.19 & 0.33 & 0.11 & 0.23 & 0.06 \\
\hline Var3 & 5.7 & 0.9 & 0.9 & 10.63 & 1.30 & 3.95 & 0.43 & 1.47 & 0.14 \\
\hline Var3/Gla & 6.3 & 0.7 & 0.7 & 5.12 & 1.34 & 2.76 & 0.50 & 1.48 & 0.19 \\
\hline Var3/GLL & 6.6 & 0.4 & 0.1 & 1.75 & 0.47 & 0.91 & 0.23 & 0.47 & 0.11 \\
\hline ATRAM & 6.4 & 0.9 & 0.1 & 2.06 & 0.40 & 1.23 & 0.22 & 0.74 & 0.12 \\
\hline
\end{tabular}




\section{FIGURES}

Figure 1. Schematic organization of pHLIP bundles: (a) PEG-2WT with 2 kDa 2-arm PEG and two WT pHLIPs, and (b) PEG-4WT with $2 \mathrm{kDa} 4$-arm PEG and four WT pHLIPs. Transitions between the three states of PEG-2WT and PEG-4WT in phosphate buffer at $\mathrm{pH} 8$ (State I), in the presence of POPC liposomes at $\mathrm{pH} 8$ (State II), and in the presence of liposomes at $\mathrm{pH} 4$ (State III) were monitored by changes of tryptophan fluorescence ( $\boldsymbol{c}$ and $\boldsymbol{d}$ ), circular dichroism $(\boldsymbol{e}$ and $\boldsymbol{f}$ ), and oriented circular dichroism (OCD) ( $g$ and $\boldsymbol{h}$ ) signals. Normalized pH-dependent steady-state transitions from State II to State III were examined by analyzing the shift in position of fluorescence spectrum maximum of PEG-2WT $(\boldsymbol{i})$ and PEG-4WT $(\boldsymbol{j})$ in the presence of physiological concentrations of calcium and magnesium ions. The data were fitted using the Henderson-Hasselbalch equation; the fitting curves and $95 \%$ confidence interval are shown by red and blue lines, respectively. 

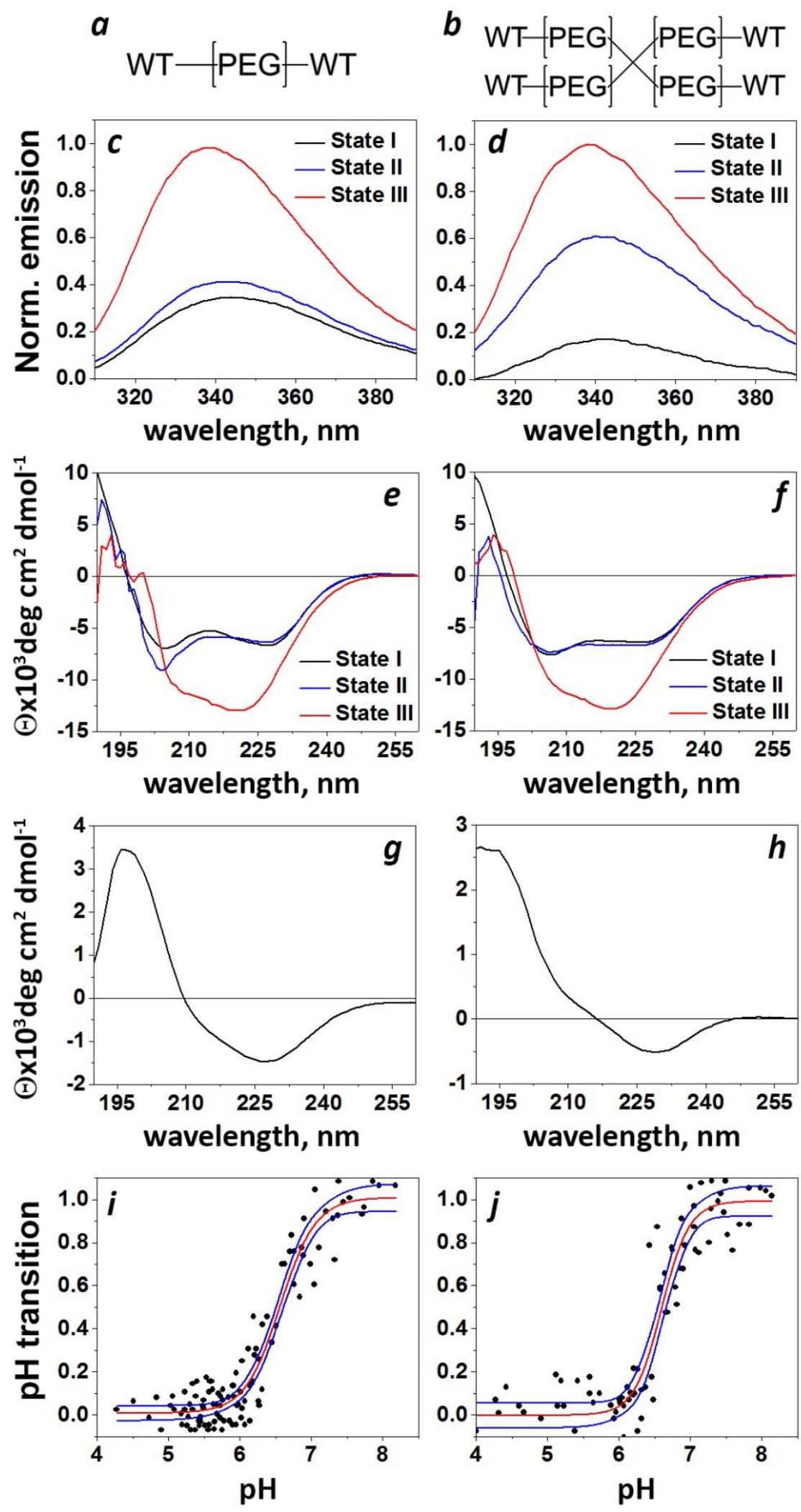
Figure 2. Ellipticity ratios of CD signals at $205 \mathrm{~nm}$ to $222 \mathrm{~nm}$ are shown for pHLIP variants in State I, II, and III (a). The values of ellipticity ratios are given in Table S3. The therapeutic index (TI) was calculated for different pHLIP-amanitin constructs as the ratio of $E C_{50}$ at $\mathrm{pH} 7.4$ to $E C_{50}$ at $\mathrm{pH} 6.0(\boldsymbol{b})$.
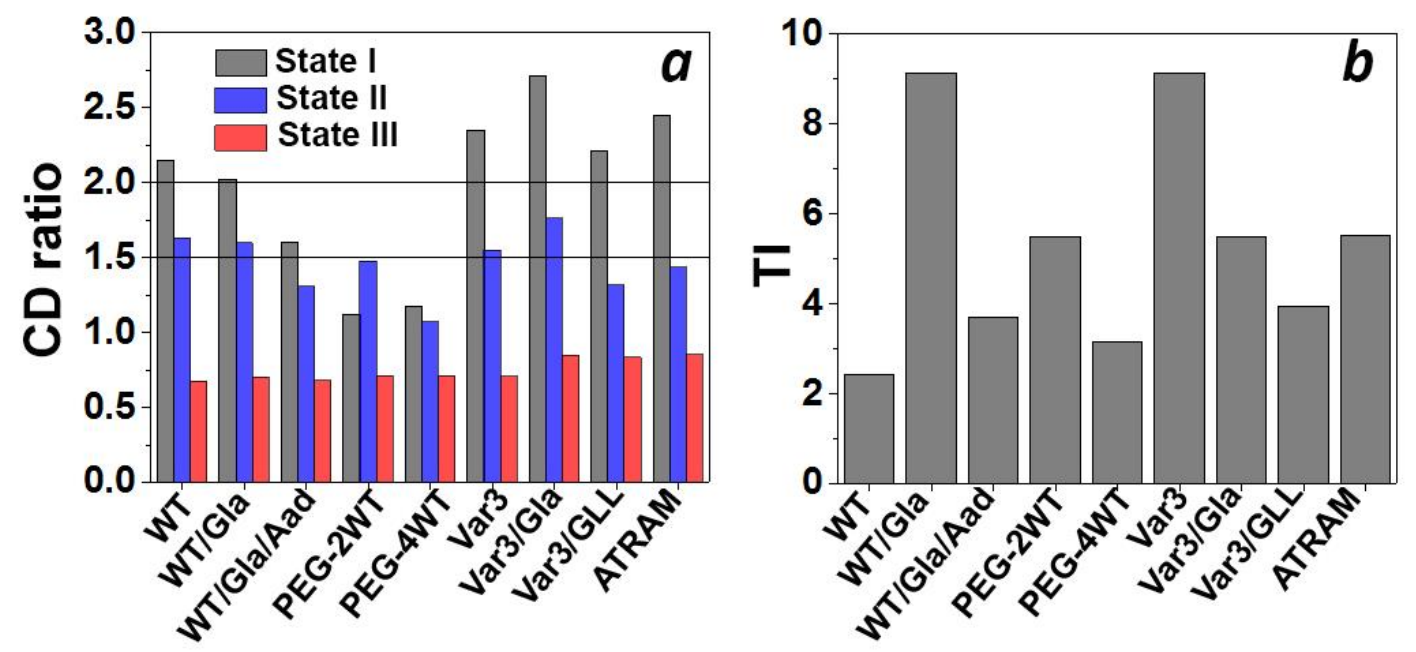
Figure 3. pH-dependent potency was defined as the difference between cancer cell viability when cells were incubated at $\mathrm{pH} 7.4$ and $\mathrm{pH} 6.0$ at varying concentrations of different pHLIP-amanitin constructs. The WT-like group is shown in $(\boldsymbol{a})$; the Var3like group and ATRAM are shown in $(\boldsymbol{b})$.
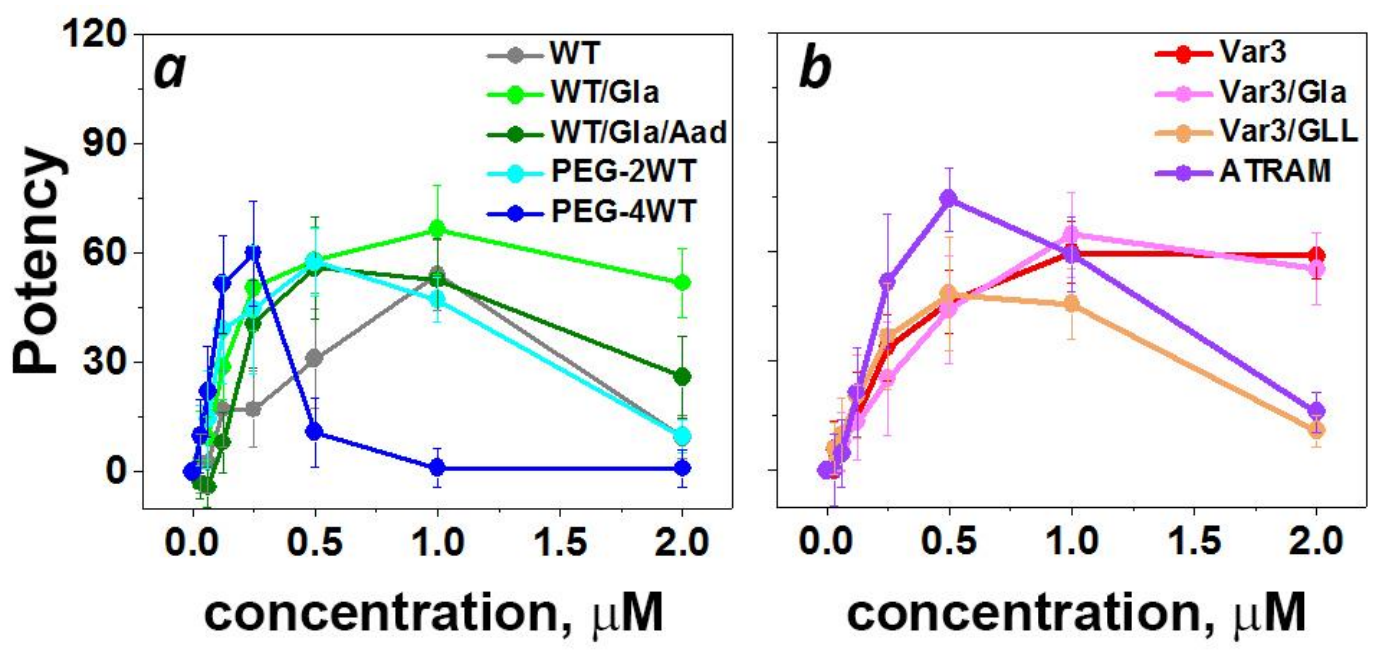
Figure 4. Normalized tumor fluorescence intensities of the AF546-pHLIP constructs are shown; the signals were normalized by the tumor intensity of AF546-WT (a). Tumor-to-muscle (T/M) (b), tumor-to-kidney (T/K) $(\boldsymbol{c})$, and tumor-to-liver (T/L) (d) fluorescence intensity ratios are shown. Statistically significant differences were determined by two-tailed unpaired Student's t-test, where * denotes $p \leq 0.05$ and ** denotes $p \leq 0.005$.
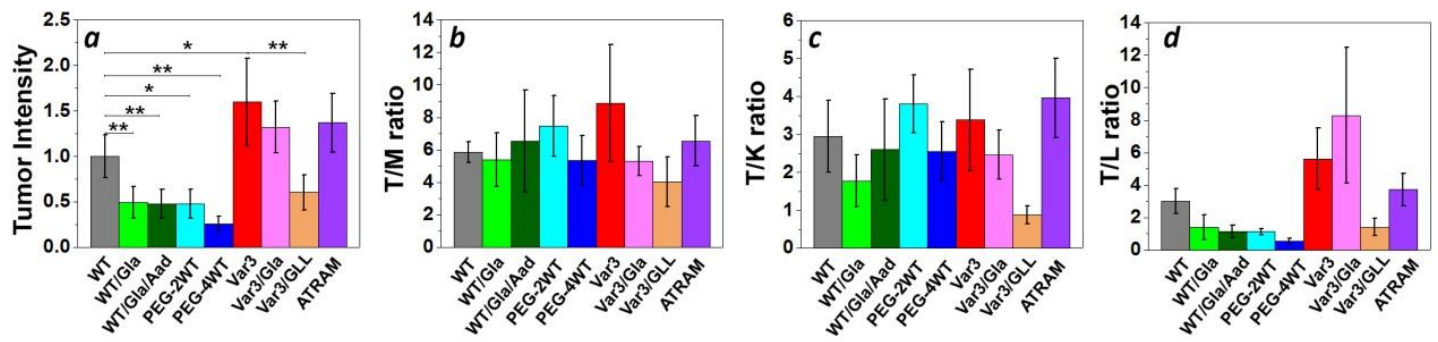


\title{
SUPPLEMENTARY INFORMATION
}

\section{New pHLIPs for the Targeted Intracellular Delivery of Cargo Molecules to Tumors}

\author{
Linden C. Wyatt, Anna Moshnikova, Troy Crawford, \\ Donald M. Engelman, Oleg A. Andreev, Yana K. Reshetnyak
}

\section{Supplementary Materials and Methods}

pHLIP characterization and pHLIP bundle synthesis. All peptides were purchased from CS Bio Co. Peptides were characterized by reversed phase high-performance liquid chromatography (RP-HPLC) using Zorbax SB-C18 and Zorbax SB-C8, $4.6 \times$ $250 \mathrm{~mm}, 5 \mu \mathrm{m}$ columns (Agilent Technology). For biophysical measurements, PEG2WT and PEG-4WT were made by conjugating either $2 \mathrm{kDa}$ bifunctional maleimidePEG-maleimide or $2 \mathrm{kDa}$ 4-arm PEG-maleimide (Creative PEGWorks) to Cys-WT via an N-terminal cysteine residue. Purification of the PEG-pHLIP constructs was conducted using RP-HPLC. Peptide concentration was calculated by absorbance at $280 \mathrm{~nm}$, where, for WT, WT/Gla, and WT/Gla/Aad, $\varepsilon_{280}=13,940 \mathrm{M}^{-1} \mathrm{~cm}^{-1}$; for Var3, Var3/Gla, and Var3/GLL, $\varepsilon_{280}=12,660 \mathrm{M}^{-1} \mathrm{~cm}^{-1}$; and for ATRAM, $\varepsilon_{280}=5,690 \mathrm{M}^{-1}$ $\mathrm{cm}^{-1}$. PEG construct concentration was presented in terms of peptide concentration, not molecular concentration. 
Liposome preparation. Small unilamellar vesicles were used as model membranes and were prepared by extrusion. 1-palmitoyl-2-oleoyl-sn-glycero-3-phosphocholine (POPC; Avanti Polar Lipids) was dissolved in chloroform at a concentration of 12.5 $\mathrm{mg} / \mathrm{mL}$, then desolvated by rotary evaporation for two hours under vacuum. The resulting POPC film was rehydrated in $10 \mathrm{mM}$ phosphate buffer at $\mathrm{pH}$ 8, either with additional calcium and magnesium ions (1.25 $\mathrm{mM}$ calcium and $0.65 \mathrm{mM}$ magnesium), or without additional ions, vortexed, and extruded fifteen times through a membrane with a pore size of $50 \mathrm{~nm}$.

Steady-state fluorescence measurements. Steady-state fluorescence spectra were measured using a PC1 spectrofluorometer (ISS) with temperature control set to 25.0 ${ }^{\circ} \mathrm{C}$. The tryptophan fluorescence was excited using an excitation wavelength of 295 $\mathrm{nm}$. Excitation and emission slits were set to $8 \mathrm{~nm}$, and excitation and emission polarizers were set to $54.7^{\circ}$ and $0.0^{\circ}$, respectively. Sample preparation was conducted 24 hours prior to experiments to allow for State II equilibration. A buffer-only sample was used as a baseline for State I, and a buffer-with-POPC-only sample was used as a baseline for States II and III.

pH dependence measurements. Measurements of $\mathrm{pH}$ dependence were taken with the PC1 spectrofluorometer by using the shift in the position of maximum of peptide fluorescence as an indication of changes of the peptide environment at varying $\mathrm{pH}$. All $\mathrm{pH}$ dependence measurements were conducted using blood physiological concentrations of free calcium and magnesium ions (1.25 and $0.65 \mathrm{mM}$, respectively). 
After the addition of hydrochloric acid, the $\mathrm{pH}$ of solutions containing $5 \mu \mathrm{M}$ peptide and $1 \mathrm{mM}$ POPC were measured using an Orion PerHecT ROSS Combination $\mathrm{pH}$ Micro Electrode and an Orion Dual Star pH and ISE Benchtop Meter (Thermo Fisher Scientific) before and after spectrum measurement to ensure equilibration. The tryptophan fluorescence spectrum at each $\mathrm{pH}$ was recorded, and the spectra were analyzed using the Protein Fluorescence and Structural Toolkit (PFAST) (42) to determine the positions of spectral maxima $\left(\lambda_{\max }\right)$. The position of $\lambda_{\max }$ was plotted as a function of $\mathrm{pH}$ and normalized, such that $\lambda_{\max }^{\text {initial }}-$ position of spectral maximum in State II was set to 1 and $\lambda_{\max }^{\text {final }}$ - position of spectral maximum in State III, was set to 0 . The normalized $\mathrm{pH}$-dependence was fit with the Henderson-Hasselbach equation (using OriginLab software) to determine the cooperativity $(n)$ and transition mid-point $(\mathrm{p} K)$ of transition of the peptide population from State II to State III:

$$
\text { Normalized } p H \text { dependence }=\frac{1}{1+10^{n(\mathrm{pH}-\mathrm{pK})}}
$$

Steady-state circular dichroism and oriented CD measurements. Steady-state CD was measured using an MOS-450 spectrometer (Bio-Logic Science Instruments) in the range of 190 to $260 \mathrm{~nm}$ with a step size of $1 \mathrm{~nm}$, and with temperature control set to $25.0{ }^{\circ} \mathrm{C}$. Samples were prepared 24 hours prior to experiments to allow for State II equilibration. A buffer-only sample was used as baseline for State I, and a buffer-withPOPC-only sample was used as baseline for States II and III.

OCD was measured using supported planar POPC bilayers prepared using a Langmuir-Blodgett system (KSV Nima). Fourteen quartz slides with $0.2 \mathrm{~mm}$ spacers 
were used; after sonicating the slides in 5\% cuvette cleaner (Contrad 70; Decon Labs) in deionized water $\left(\geq 18.2 \mathrm{M} \Omega \mathrm{cm}\right.$ at $25{ }^{\circ} \mathrm{C}$; Milli-Q Type 1 Ultrapure Water System, EMD Millipore) for fifteen minutes and rinsing with deionized water, the slides were immersed and sonicated for ten minutes in 2-propanol, sonicated again for ten minutes in acetone, sonicated a final time in 2-propanol for ten minutes, and rinsed thoroughly with deionized water. Lastly, the slides were immersed in a 3:1 solution of sulfuric acid to hydrogen peroxide for five minutes and rinsed three times in deionized water. The slides were stored in deionized water until they were used. POPC bilayers were deposited on the fourteen slides using a Langmuir-Blodgett minitrough: a $2.5 \mathrm{mg} / \mathrm{mL}$ solution of POPC in chloroform was spread on the subphase (deionized water) and the chloroform was allowed to evaporate for fifteen minutes, after which the POPC monolayer was compressed to $32 \mathrm{mN} / \mathrm{m}$. A lipid monolayer was deposited on the slides by retrieving them from the subphase, after which a solution of $10 \mu \mathrm{M}$ peptide and $500 \mu \mathrm{M}$ of $50 \mathrm{~nm}$ POPC liposomes at $\mathrm{pH} 4$ was added to the slides, resulting in the creation of the supported bilayer by fusion between the monolayer on the slides and the peptide-laden lipid vesicles. After incubation for six hours at $100 \%$ humidity, the slides were rinsed with buffer solution to remove excess liposomes, and the spaces between the cuvettes were filled with buffer at $\mathrm{pH} 4$. Measurements were taken at three points during the experiment: immediately after the addition of the peptide/lipid solution $(0 \mathrm{~h})$, after the slides were rinsed to remove excess liposomes following the six-hour incubation time $(6 \mathrm{~h})$, and after an additional twelve-hour incubation time and rinse with buffer $(18 \mathrm{~h})$; these measurements were recorded on the MOS-450 spectrometer with sampling times of two seconds at each wavelength. Control 
measurements were conducted using a peptide solution between slides without supported bilayers and in the presence of POPC liposomes.

Kinetics measurements. Stopped-flow fluorescence measurements were made using an SFM-300 mixing system (Bio-Logic Science Instruments) in conjunction with the MOS-450 spectrometer. All solutions were degassed for fifteen minutes prior to loading into the stopped-flow system. pHLIP variants were incubated with POPC for 24 hours prior to the experiment to reach State II equilibrium, and insertion was induced by mixing equal volumes of pHLIP/POPC solutions with hydrochloric acid diluted to ensure a $\mathrm{pH}$ drop from $\mathrm{pH} 8$ to $\mathrm{pH} 4$. Kinetics data were fit by one-, two-, three-, or four-state exponential models in OriginLab.

Amanitin pHLIP conjugates. $\alpha$-amanitin (Sigma-Aldrich) was conjugated to succinimidyl 3-(2-piridyldithio)propionate) (SPDP; Thermo Fisher Scientific), followed by purification and conjugation of the SPDP-amanitin to the C-terminal cysteine residues of pHLIP peptides. For synthesis of PEG-2WT-amanitin and PEG4WT-amanitin, Lys-WT-Cys with N-terminal lysine and C-terminal cysteine residues was used, and the Lys-WT-SPDP-amanitin was conjugated to dibenzocyclooctynesulfo-N-hydroxysuccinimidyl ester (DBCO-NHS ester; Sigma-Aldrich), resulting in DBCO-WT-SPDP-amanitin. Finally, 2-arm or 4-arm PEG-azide (Creative PEGWorks) was conjugated to DBCO-WT-SPDP-amanitin, resulting in PEG-DBCOWT-SPDP-amanitin, with a cleavable disulfide bond present in SPDP, between the peptide and amanitin cargo. Construct concentration was calculated by absorbance at 
$310 \mathrm{~nm}$, where, for $\alpha$-amanitin, $\varepsilon_{310}=13,000 \mathrm{M}^{-1} \mathrm{~cm}^{-1}$. Construct concentration was presented in terms of peptide/amanitin concentration. Purification was conducted using RP-HPLC. Zorbax SB-C18 columns $(9.4 \times 250 \mathrm{~mm}, 5 \mu \mathrm{m}$; Agilent Technologies) were used for all peptide-amanitin conjugates other than ATRAMamanitin, PEG-2WT-amanitin, and PEG-4WT-amanitin, for which Zorbax SB-C8 columns $(9.4 \times 250 \mathrm{~mm}, 5 \mu \mathrm{m}$; Agilent Technologies) were used.

Cell proliferation assay. Human cervix adenocarcinoma cells (HeLa; American Type Culture Collection) were authenticated, stored according to the instructions of the supplier, and used within three months of frozen aliquot resuscitation. Cells were cultured in Dulbecco's modified Eagle's medium (DMEM; Sigma-Aldrich) at pH 7.4 with $4.5 \mathrm{~g} / \mathrm{L}$ D-glucose, supplemented with $10 \%$ heat-inactivated fetal bovine serum (FBS; Sigma-Aldrich) and $10 \mu \mathrm{g} / \mathrm{mL}$ ciprofloxacin (Sigma-Aldrich), in a humidified atmosphere of $5 \% \mathrm{CO}_{2}$ and $95 \%$ air at $37{ }^{\circ} \mathrm{C}$. The $\mathrm{pH} 6.0$ medium was prepared by mixing $13.3 \mathrm{~g}$ of dry DMEM in $1 \mathrm{~L}$ of deionized water. HeLa cells were loaded in the wells of 96-well plates (5,000 cells/well) and incubated overnight. The standard growth medium was replaced with medium without FBS, at $\mathrm{pH} 6.0$ or 7.4 , containing increasing amounts of pHLIP-amanitin conjugates (from 0 to $2.0 \mu \mathrm{M}$ ). Treatment with amanitin alone for two hours and at concentrations up to $2 \mu \mathrm{M}$ does not induce cell death (35). After two-hour incubation with the pHLIP-amanitin conjugates, the constructs were removed and replaced with standard growth medium. Cell viability was assessed after 48 hours using the CellTiter $96 \mathrm{AQ}_{\text {ueous }}$ One Solution Cell Proliferation Assay (Promega); the colorimetric reagent was added to cells for one 
hour, followed by absorption measurement at $490 \mathrm{~nm}$. All samples were prepared in triplicate, and each experiment was repeated between 3 and 6 times. All obtained cell proliferation data were normalized by corresponding controls (non-treated cells). There was no difference in the viability of cells incubated with media, without construct, at $\mathrm{pH} 7.4$ and $\mathrm{pH} 6.0$; therefore, the role of $\mathrm{pH}$ was excluded from the consideration. Normalized cell viability data obtained in different experiments were averaged and presented in terms of the logarithm of dose of pHLIP-amanitin constructs. The dose-response function was used for fitting the obtained data (Figure S2) (OriginLab):

Cell Viability $=A_{b}+\frac{A_{t}-A_{b}}{1+10^{p\left(\log x_{0}-x\right)}}$

where $A_{b}$ and $A_{t}$ are the bottom and the top asymptotes, respectively. The top asymptote was set as constant $(100 \%)$ while for the bottom asymptote we allowed small variations in the range of 0 to $10 \% . p$ is the slope (cooperativity parameter) and $\log x_{0}$ is the center of the transition (i.e., the concentration for half response) which is used to calculate the $E C_{20}, E C_{50}, E C_{80}$ values:

$$
\begin{aligned}
& E C_{20}=10^{\left(\log x_{0}+^{\log 0.25} / p\right)} \\
& E C_{50}=10^{\log x_{0}} \\
& E C_{80}=10^{\left(\log x_{0}+{ }^{\log 4} / p\right)}
\end{aligned}
$$


Therapeutic index (TI) was calculated according to the equation:

$T I=\frac{E C_{50}^{\mathrm{pH} 7.4}}{E C_{50}^{\mathrm{p} H 6.0}}$

Additionally, the cytotoxicity of the PEG-2WT and PEG-4WT constructs without amanitin was tested: these experiments demonstrated no cytotoxicity at physiological or low $\mathrm{pH}$ at treatment concentrations up to $10 \mu \mathrm{M}$.

Fluorescent pHLIP conjugates. Alexa Fluor 546 (AF546) $\mathrm{C}_{5}$ maleimide (Thermo Fisher Scientific) was conjugated to the N-terminal cysteine residues of WT, Var3, Var3/Gla, and ATRAM. AF546 NHS Ester (Thermo Fisher Scientific) was conjugated to the N-terminal lysine residues of WT/Gla, WT/Gla/Aad, and Var3/GLL. For PEG2WT and PEG-4WT, Cys-WT-Lys, with N-terminal cysteine and C-terminal lysine residues, was used, and was first conjugated to 2-arm maleimide-PEG-maleimide or 4arm PEG-maleimide resulting in PEG-WT-Lys. Then, AF546 NHS Ester was conjugated to the C-terminal lysine residue, resulting in 2-arm and 4-arm PEG-pHLIP constructs with C-terminal AF546 fluorophores. Construct concentration was calculated by absorbance at $554 \mathrm{~nm}$, where, for AF546, $\varepsilon_{554}=93,000 \mathrm{M}^{-1} \mathrm{~cm}^{-1}$. Construct concentration was presented in terms of AF546/peptide concentration, not molecular concentration. Purification was conducted using RP-HPLC for all peptides other than PEG-4WT-AF546, which was purified via Amicon Ultra MWCO $10 \mathrm{kDa}$ centrifugal filter (Sigma-Aldrich). Zorbax SB-C18 columns $(9.4 \times 250 \mathrm{~mm}, 5 \mu \mathrm{m}$; 
Agilent Technologies) were used for all AF546-peptide conjugates except AF546ATRAM and PEG-2WT-AF546, for which Zorbax SB-C8 columns $(9.4 \times 250 \mathrm{~mm}, 5$ $\mu \mathrm{m}$; Agilent Technologies) were used.

Ex vivo imaging. All animal studies were conducted according to the animal protocol AN04-12-011 approved by the Institutional Animal Care and Use Committee at the University of Rhode Island, in compliance with the principles and procedures outlined by the National Institutes of Health for the care and use of animals. Mouse mammary cells (4T1; American Type Culture Collection) were subcutaneously implanted in the right flank $\left(8 \times 10^{5}\right.$ cells $\left./ 0.1 \mathrm{~mL} / \mathrm{flank}\right)$ of adult female BALB/cAnNHsd mice (Envigo). When tumors reached approximately $5-6 \mathrm{~mm}$ in diameter, single tail vein injections of $100 \mu \mathrm{L}, 40 \mu \mathrm{M}$ fluorophore-pHLIP solutions in PBS were performed. Mice were euthanized 4 or 24 hours after injection, and necropsy was immediately performed. Tumors and major organs were cut in half and imaged using an FX Kodak in-vivo image station connected to an Andor CCD camera. Mean surface fluorescence intensity of tumor, tissue and organs was obtained via analysis of fluorescence images in ImageJ (NIH) (43). The corresponding autofluorescence signal was subtracted to obtain the net fluorescence intensities used in the study. Autofluorescence was calculated after imaging tumors, tissue, and organs collected from mice with no injection of fluorescent pHLIP constructs. 


\section{SUPPLEMENTARY TABLES}

Table S1. List of pHLIP sequences used in the study.

\begin{tabular}{|l|l|}
\hline \multicolumn{1}{|c|}{ Peptide } & \multicolumn{1}{c|}{ Sequence } \\
\hline Cys-WT & ACEQNPIYWARYADWLFTTPLLLLDLALLVDADEGT \\
WT-Cys & AEQNPIYWARYADWLFTTPLLLLDLALLVDADEGCT \\
Lys-WT-Cys & Ac-AKEQNPIYWARYADWLFTTPLLLLDLALLVDADECT \\
Lys-WT/Gla-Cys & Ac-AKEQNPIYWARYAGlaWLFTTPLLLLDLALLVDADECT \\
Lys-WT/Gla/Aad-Cys & Ac-AKEQNPIYWARYAGlaWLFTTPLLLLAadLALLVDADECT \\
Cys-Var3 & ACDDQNPWRAYLDLLFPTDTLLLDLLWA \\
Var3-Cys & ADDQNPWRAYLDLLFPTDTLLLLLWCA \\
Cys-Var3/Gla & ACDDQNPWRAYLGlaLLFPTDTLLLDLLWG \\
Var3/Gla-Cys & ADDQNPWRAYLGlaLLFPTDTLLLDLLWCG \\
Lys-Var3/GLL-Cys & Ac-GKEEQNPWLGAYLDLLFPLELLGLLELGLWCG \\
Cys-ATRAM & ACGLAGLAGLLGLEGLLGLPLGLLEGLWLGLELEGN \\
ATRAM-Cys & GLAGLAGLLGLEGLLGLPLGLLEGLWLGLELEGNCA \\
\hline
\end{tabular}


Table S2. Molecular weights (MW) of peptides, and retention times and corresponding percentages of acetonitrile at elution for 30 min method of $25-80 \%$ gradient of acetonitrile/0.05\% TFA in water/0.05\% TFA used in C18 and C8 columns. ${ }^{\dagger}$ Peptide eluted during washing with acetonitrile, after the completion of the gradient.

\begin{tabular}{|c|c|c|c|c|c|}
\hline \multirow{2}{*}{ Peptide } & \multirow{2}{*}{ MW (Da) } & \multicolumn{2}{|c|}{$\begin{array}{c}\text { Zorbax SB-C18 } \\
(4.6 \times 250 \mathrm{~mm}, 5 \mu \mathrm{m})\end{array}$} & \multicolumn{2}{|c|}{$\begin{array}{c}\text { Zorbax SB-C8 } \\
(4.6 \times 250 \mathrm{~mm}, 5 \mu \mathrm{m})\end{array}$} \\
\hline & & $\begin{array}{l}\text { Retention } \\
\text { Time (min) }\end{array}$ & $\%$ Acetonitrile & $\begin{array}{c}\text { Retention } \\
\text { Time (min) }\end{array}$ & $\%$ Acetonitrile \\
\hline Cys-WT & 4111.7 & 21.6 & $64.6 \%$ & 20.6 & $62.8 \%$ \\
\hline Lys-WT-Cys & 4224.9 & 21.6 & $64.6 \%$ & 20.4 & $62.4 \%$ \\
\hline Lys-WT/Gla-Cys & 4283.1 & 22.3 & $65.9 \%$ & 21.2 & $63.9 \%$ \\
\hline Lys-WT/Gla/Aad-Cys & 4310.9 & 22.8 & $66.8 \%$ & 21.7 & $64.8 \%$ \\
\hline Cys-Var3 & 3292.8 & 19.7 & $61.1 \%$ & 19.6 & $60.9 \%$ \\
\hline Cys-Var3/Gla & 3333.8 & 19.9 & $61.5 \%$ & 19.7 & $61.1 \%$ \\
\hline Lys-Var3/GLL-Cys & 3643.2 & 28.0 & $76.2 \%$ & 26.0 & $72.7 \%$ \\
\hline Cys-ATRAM & 3516.2 & $30.6^{\dagger}$ & $93.0 \%$ & 27.2 & $74.9 \%$ \\
\hline
\end{tabular}


Table S3. Positions of maxima of tryptophan fluorescence spectra $\left(\lambda_{\max }\right)$ and ratios of ellipticity at $205 \mathrm{~nm}$ to $222 \mathrm{~nm}$ of pHLIP constructs in States I, II, and III are given.

\begin{tabular}{|l|c|c|c|c|c|c|}
\hline \multirow{2}{*}{ Construct } & \multicolumn{3}{|c|}{$\lambda_{\max }(\mathbf{n m})$} & \multicolumn{3}{c|}{ Ellipticity at 205/222 $\mathbf{~ n m}$} \\
\cline { 2 - 7 } & State I & State II & State III & State I & State II & State III \\
\hline WT & 351.6 & 347.4 & 340.0 & 1.61 & 2.16 & 0.68 \\
WT/Gla & 348.2 & 347.1 & 338.2 & 2.02 & 1.60 & 0.70 \\
WT/Gla/Aad & 347.5 & 346.7 & 338.3 & 1.60 & 1.31 & 0.68 \\
PEG-2WT & 341.8 & 341.2 & 336.6 & 1.12 & 1.48 & 0.71 \\
PEG-4WT & 344.0 & 343.4 & 338.9 & 1.18 & 1.07 & 0.71 \\
Var3 & 350.2 & 346.8 & 339.9 & 2.35 & 1.55 & 0.71 \\
Var3/Gla & 351.4 & 345.7 & 339.2 & 2.72 & 1.76 & 0.85 \\
Var3/GLL & 349.3 & 343.3 & 341.2 & 2.21 & 1.32 & 0.84 \\
ATRAM & 345.5 & 341.4 & 333.0 & 2.45 & 1.44 & 0.85 \\
\hline
\end{tabular}


Table S4. Fluorescence intensity obtained by ex vivo imaging of tumor, tissue, and organs 4 hours after a single IV administration of the Alexa Fluor 546-pHLIP constructs (data obtained at 24 hours post-injection are shown for PEG-2WT and PEG-4WT). Values of tissue autofluorescence are provided in the last row. Sample size $(n)$ is given in the last column for each construct.

\begin{tabular}{|c|c|c|c|c|c|c|c|}
\hline \multirow{2}{*}{ Construct } & \multicolumn{7}{|c|}{ Total Fluorescence Intensity in tumor, muscle and organs (a.u.) } \\
\hline & Tumor & Muscle & Kidney & Liver & Lungs & Spleen & $n$ \\
\hline WT & $2335.7 \pm 447.1$ & $726.8 \pm 58.5$ & $988.5 \pm 91.0$ & $998.7 \pm 142.0$ & $545.8 \pm 32.6$ & $447.0 \pm 31.7$ & 5 \\
\hline WT/Gla & $1372.1 \pm 331.7$ & $591.2 \pm 73.2$ & $880.2 \pm 126.5$ & $1102.1 \pm 239.6$ & $456.2 \pm 68.6$ & $387.9 \pm 63.3$ & 13 \\
\hline WT/Gla/Aad & $1336.6 \pm 304.5$ & $559.6 \pm 53.5$ & $726.6 \pm 149.9$ & $1156.4 \pm 120.8$ & $448.5 \pm 79.9$ & $374.1 \pm 45.0$ & 13 \\
\hline PEG-2WT & $1443.4 \pm 178.5$ & $545.3 \pm 42.2$ & $602.7 \pm 79.2$ & $1267.1 \pm 146.2$ & $462.0 \pm 28.5$ & $450.7 \pm 17.3$ & 5 \\
\hline PEG-4WT & $912.8 \pm 159.5$ & $495.5 \pm 23.0$ & $523.4 \pm 52.5$ & $1209.7 \pm 100.7$ & $431.0 \pm 54.9$ & $403.7 \pm 30.0$ & 7 \\
\hline Var3 & $3474.3 \pm 924.2$ & $760.7 \pm 68.3$ & $1263.9 \pm 136.5$ & $904.8 \pm 93.4$ & $564.3 \pm 130.1$ & $335.6 \pm 45.0$ & 7 \\
\hline Var3/Gla & $2948.9 \pm 540.6$ & $889.3 \pm 144.7$ & $1390.0 \pm 247.5$ & $702.9 \pm 140.9$ & $784.5 \pm 248.7$ & $379.2 \pm 47.8$ & 5 \\
\hline Var3/GLL & $1577.9 \pm 364.2$ & $694.2 \pm 27.5$ & $1690.8 \pm 431.8$ & $1194.3 \pm 147.1$ & $520.2 \pm 18.6$ & $442.8 \pm 37.4$ & 5 \\
\hline ATRAM & $3039.5 \pm 620.9$ & $813.3 \pm 105.4$ & $1009.8 \pm 183.9$ & $1073.2 \pm 146.3$ & $716.6 \pm 109.0$ & $421.3 \pm 35.3$ & 9 \\
\hline PEG-2WT (24 h) & $894.9 \pm 151.8$ & $486.3 \pm 19.8$ & $525.1 \pm 37.6$ & $753.6 \pm 54.0$ & $547.2 \pm 64.8$ & $346.3 \pm 11.0$ & 5 \\
\hline PEG-4WT (24 h) & $663.5 \pm 121.1$ & $440.1 \pm 7.7$ & $464.2 \pm 35.1$ & $726.7 \pm 69.6$ & $482.8 \pm 48.5$ & $331.8 \pm 4.4$ & 5 \\
\hline Autofluorescence & $426.9 \pm 15.7$ & $402.7 \pm 11.5$ & $325.6 \pm 30.8$ & $354.7 \pm 28.3$ & $392.7 \pm 32.7$ & $296.1 \pm 23.2$ & 12 \\
\hline
\end{tabular}


Table S5. Tumor-to-muscle (T/M), tumor-to-kidney (T/K), and tumor-to-liver (T/L) ratios presented in Figures $4 b, 4 c$, and $4 d$, respectively.

\begin{tabular}{|l|c|c|c|}
\hline \multicolumn{1}{|c|}{ Construct } & T/M & T/K & T/L \\
\hline WT & $5.87 \pm 0.64$ & $2.95 \pm 0.95$ & $3.03 \pm 0.76$ \\
WT/Gla & $5.39 \pm 1.65$ & $1.77 \pm 0.68$ & $1.40 \pm 0.76$ \\
WT/Gla/Aad & $6.54 \pm 3.15$ & $2.59 \pm 1.33$ & $1.14 \pm 0.37$ \\
PEG-2WT & $7.48 \pm 1.86$ & $3.80 \pm 0.77$ & $1.13 \pm 0.19$ \\
PEG-4WT & $5.34 \pm 1.53$ & $2.55 \pm 0.78$ & $0.57 \pm 0.17$ \\
Var3 & $8.86 \pm 3.61$ & $3.38 \pm 1.34$ & $5.62 \pm 1.90$ \\
Var3/Gla & $5.30 \pm 0.89$ & $2.46 \pm 0.64$ & $8.29 \pm 4.19$ \\
Var3/GLL & $4.03 \pm 1.54$ & $0.87 \pm 0.23$ & $1.42 \pm 0.55$ \\
ATRAM & $6.56 \pm 1.55$ & $3.96 \pm 1.05$ & $3.72 \pm 1.01$ \\
\hline
\end{tabular}


Table S6. The membrane-inserted populations of different pHLIP variants were calculated using the $\mathrm{pH}$ dependence parameters $\mathrm{p} K$ and $n$ (Table 1).

\begin{tabular}{|c|c|c|c|c|c|c|c|c|c|}
\hline $\mathbf{p H}$ & $\mathbf{W T}$ & WT/Gla & WT/Gla/Aad & PEG-2WT & PEG-4WT & Var3 & Var3/Gla & Var3/GLL & ATRAM \\
\hline $\mathbf{7 . 4}$ & $2 \%$ & $2 \%$ & $7 \%$ & $4 \%$ & $2 \%$ & $3 \%$ & $15 \%$ & $32 \%$ & $11 \%$ \\
$\mathbf{7 . 2}$ & $5 \%$ & $3 \%$ & $13 \%$ & $8 \%$ & $5 \%$ & $4 \%$ & $19 \%$ & $37 \%$ & $16 \%$ \\
$\mathbf{7 . 0}$ & $11 \%$ & $6 \%$ & $22 \%$ & $16 \%$ & $12 \%$ & $6 \%$ & $24 \%$ & $41 \%$ & $22 \%$ \\
$\mathbf{6 . 8}$ & $22 \%$ & $11 \%$ & $34 \%$ & $30 \%$ & $27 \%$ & $9 \%$ & $31 \%$ & $45 \%$ & $30 \%$ \\
$\mathbf{6 . 5}$ & $50 \%$ & $26 \%$ & $58 \%$ & $60 \%$ & $62 \%$ & $16 \%$ & $42 \%$ & $52 \%$ & $45 \%$ \\
$\mathbf{6 . 2}$ & $78 \%$ & $50 \%$ & $78 \%$ & $84 \%$ & $88 \%$ & $26 \%$ & $54 \%$ & $59 \%$ & $60 \%$ \\
$\mathbf{6 . 0}$ & $89 \%$ & $67 \%$ & $87 \%$ & $92 \%$ & $95 \%$ & $35 \%$ & $62 \%$ & $63 \%$ & $70 \%$ \\
\hline
\end{tabular}


Fig. S1. Three-state tryptophan fluorescence ( $A$ and $D)$, circular dichroism $(B$ and $E)$, and $\mathrm{pH}$ dependence plots $(C$ and $F)$ are shown for Var3/Gla $(A-C)$ and Var3/GLL ( $D$ F) pHLIP variants. Transitions between the three folding states of Var3/Gla and Var3/GLL pHLIP variants in phosphate buffer at $\mathrm{pH} 8$ (State I), in the presence of POPC liposomes at pH 8 (State II), and in the presence of liposomes at pH 4 (State III) were monitored by changes of tryptophan fluorescence $(A$ and $D)$, and circular dichroism ( $B$ and $E$ ) signals. pH-dependent steady-state transitions from State II to State III were examined by analyzing the shift of the position of tryptophan fluorescence spectrum maxima of Var3/Gla $(C)$ and $\operatorname{Var} 3 / \mathrm{GLL}(F)$ constructs in the presence of physiological concentrations of calcium and magnesium ions. The data were fitted using the Henderson-Hasselbalch equation; the fitting curves and 95\% confidence interval are shown by red and blue lines, respectively.
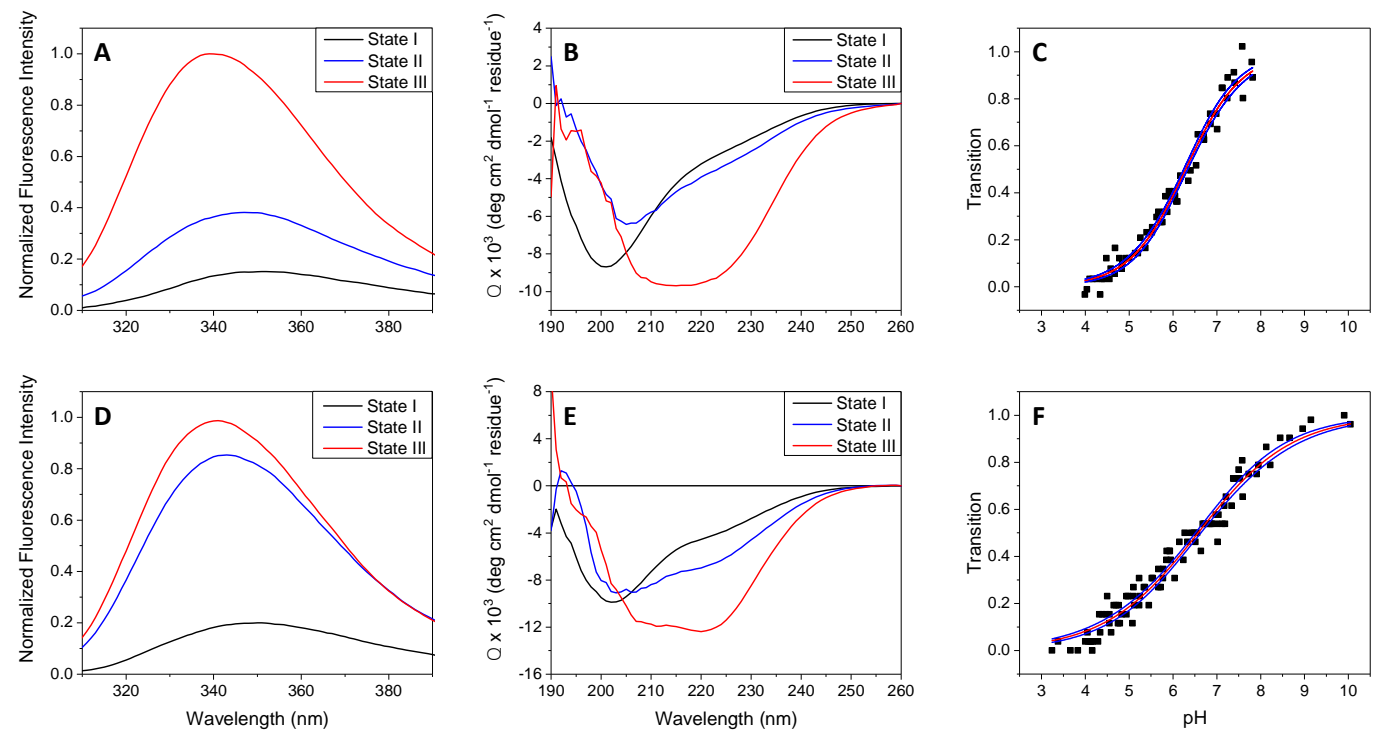
Fig. S2. Cell viability data were obtained after treatment of HeLa cells with pHLIPamanitin constructs for 2 hours at $\mathrm{pH} 7.4$ and $\mathrm{pH}$ 6.0, followed by removal of the constructs, transferal of cells to normal cell culture media, and assessment of cell death at 48 hours by MTS assay.
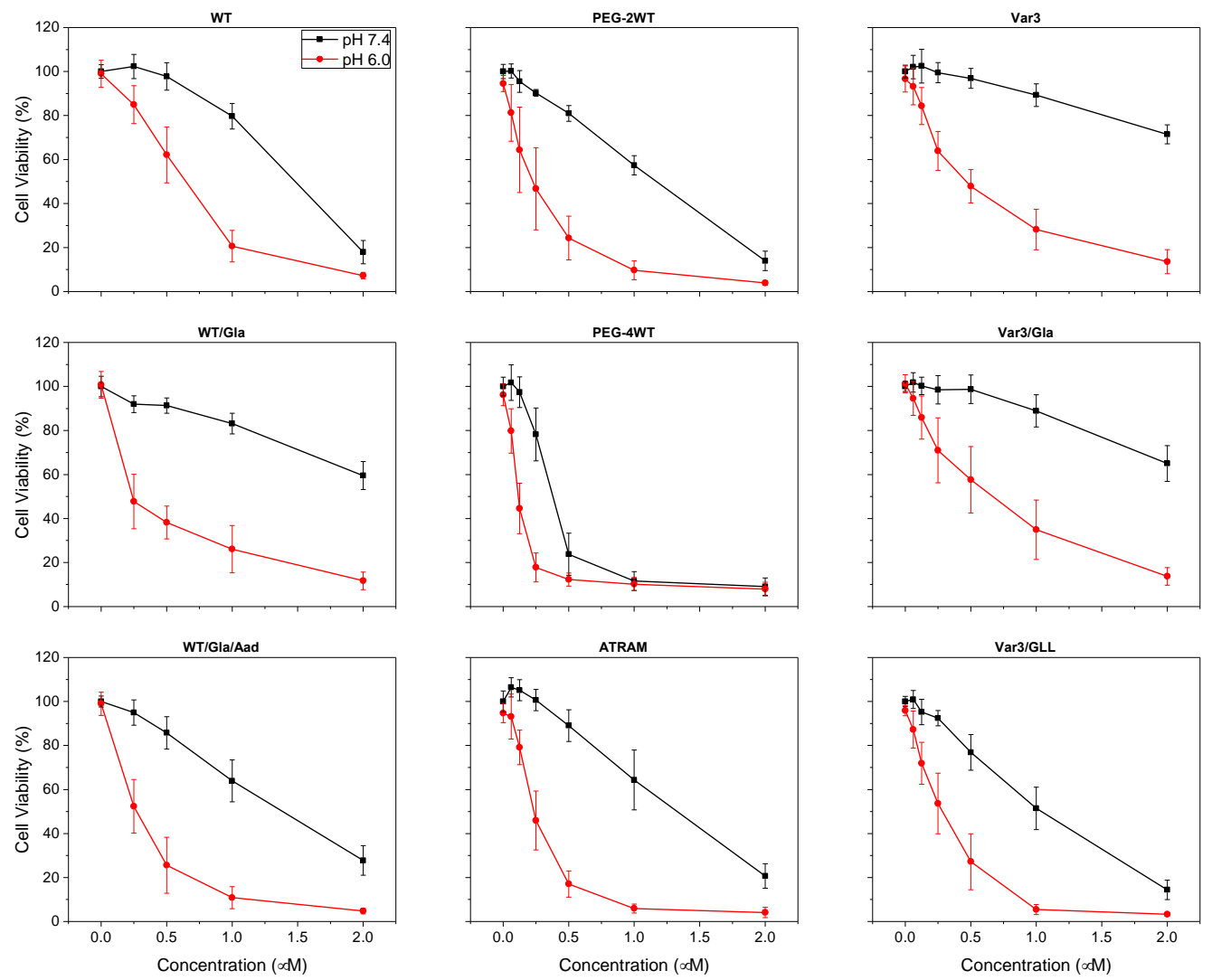